

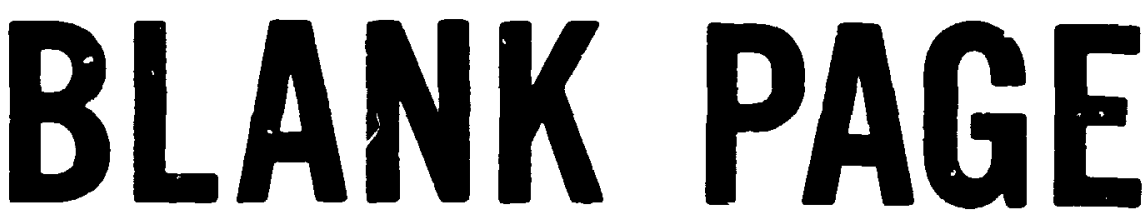


Printed in the United States of America. Avalable from N stional Tectrical information Survice

U.S. Department of Commerce 5285 Port Royal Road. Soringtield, Virgınıa 22161

Price: Printed Copy S8.75; Microfiche \$2.25

This eport was prepered as en account of work sponsored by the United : ivtes Gremenment. Neither the United States nor the Enwer Rewereh and Dewlopment Adrisustration. nor eny of their emplovess. nor any of theip contrectors. subcintractors, or ther emploves, rbes any werranty. express or impled, or

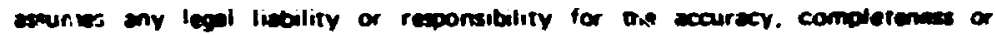
usefu nese of eny informetion, appuratus product or procses cisclasers, or repreants that it use would not infringe provety owned rents. 


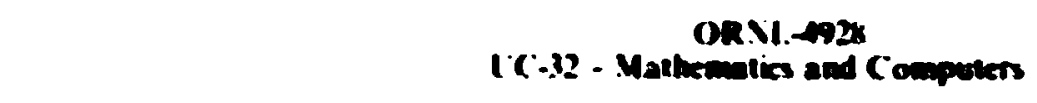

Cimatract W. 2:-7us-rac-20

COMPI IFR SCIF XCES ISTISKOS

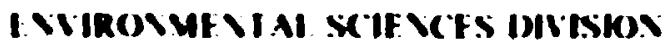

\title{
MATERIAL TRANSPORT THROUGH POROUS MEDIA: A FINITE-ELEMENT GALERKIN MODEL
}

\author{
1. O. Dreguid \\ Fnvironmental Sciences Disision \\ M. Meries \\ Computer Sciences Division
}

Environmental Sciences Division Publication 733

MARCH 1976

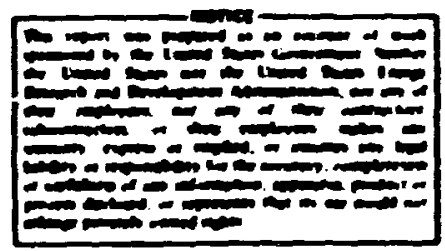

OAK RIDGE NATIONAI. LABORATORY

Oak Ridje. Tennerwer 37830 operaled by

UNION CARBIDE CORPORATION

for the

U.S. ENERGY RESEARCH A.ND DEVELOPMENT ADM'NISTRATION

DISTRIBUTION OF THIS DOCWMENT IS UMUMUTED 
CUNIt vis

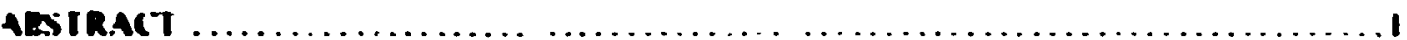

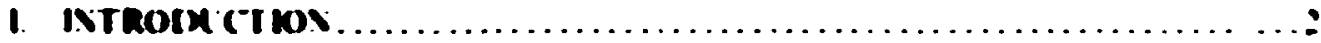

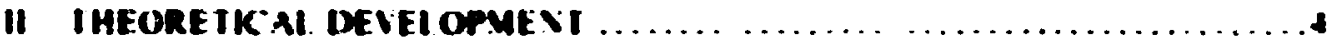

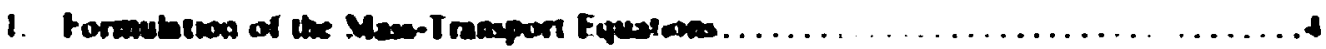

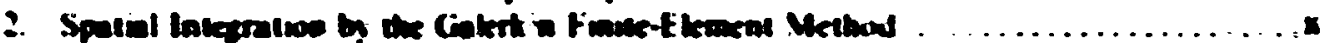

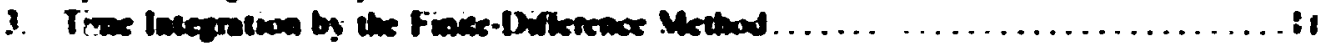

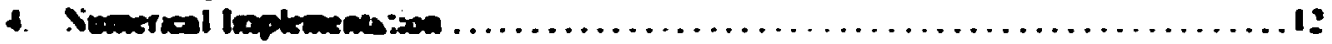

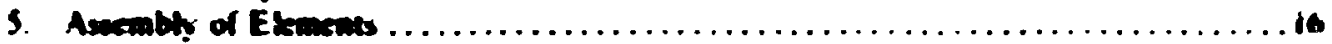

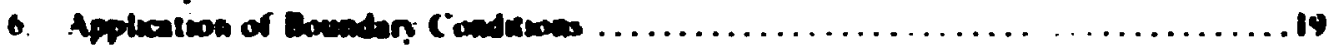

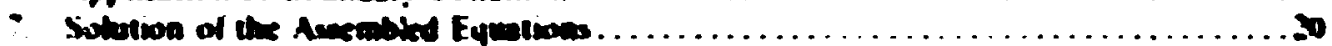

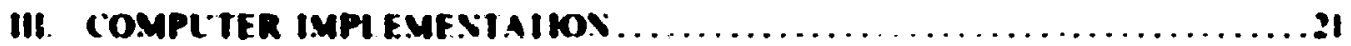

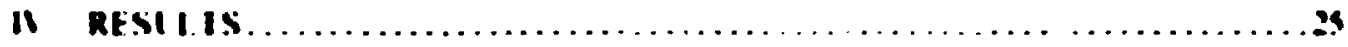

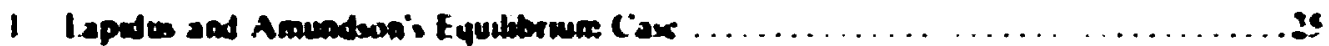

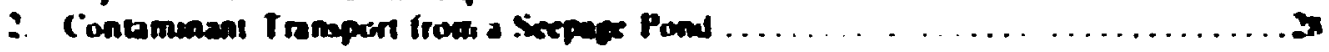

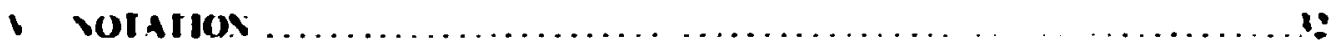

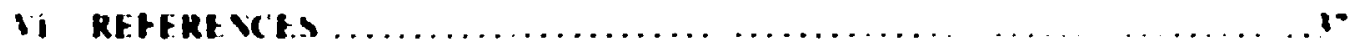

\section{APPENISKFS}

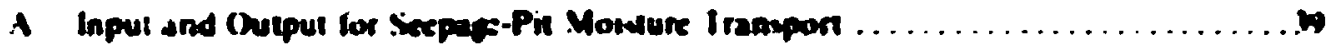

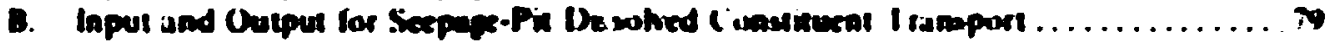

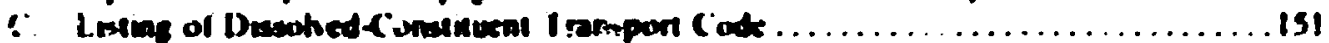

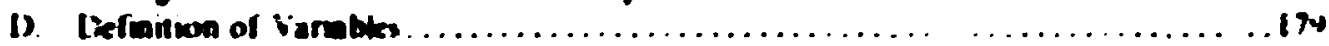

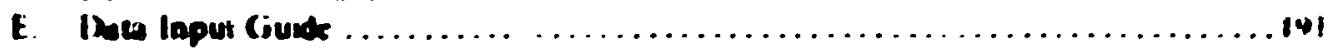




\section{I.ISI OF FKit RFS}

Figure I The i-th Finite Element in Gibbal and I cmal Cimordinates.

Fygure 2. Ecample Problem. (a) Ekement representation of the sacie using ghotal numbering sistem. (b) Local and global numbering xhernes for ekment matrix / (]).

Fiqure 3. Complete and Banded Matrix [C] for the Example Probkem.

Figure 4. Flow Chant of Dissolied-Constituent Iransport Progrem.

Figure 5. Flaw Chan for the Inure-lieration loop.

Fiqure 6. Plots Comparme Numerical and Anahtical Results. Numerical values nete otheained using the computer code dexcribed in this report. Whereas analytkal values were obcained frivn the formule gnen b! lapidus and Amundsin

Higure ?. (a) Spatel Inscretwation. (b) Water Tabte $(h=0)$ and Intal Head i H) Configurations lor Moisture Iranspon from a Seepage Pond to a Stream.

Figure X. Hydraulic Conductin it! and Sont-Moisture Characterissics of a Hypothetical Sand! Sinl.

Fiqure 9. Dispersion in ilight? Permeable Sand of a Dissohed Constituent with a [Distributiunn Coefficient of $100 \mathrm{~cm} \mathrm{gr}$. 


\section{ACKMOHIERKMENTS}

Approxumately onc man-icar was expended on the compuler model described in thes report. Ite tinamial support came :iem the folkow ine two agencies:

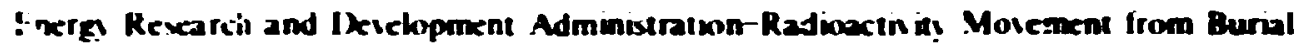
(irounds in Melion and whike (Dak Valleys

Datmenal Sikence Foundatum-Research Applied to National Need Enviroanental Aspects of I rex Contaminants Program I nder NSF Interagency Agreemeat No. AG-369.

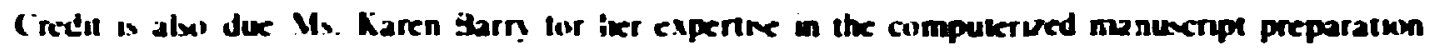
exhnuyer uxed in this repors 


\title{
MATERIAL TRANSPORT THROUGH POROUS MEDIA: \\ A FNTTE-ELEMENT GALERKIN MODEL
}

\author{
J. O. Duguid and $\mathbf{M}$. Reeves
}

\begin{abstract}
ASTRACT

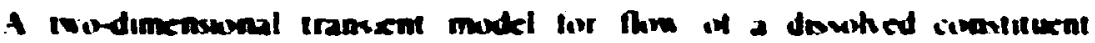

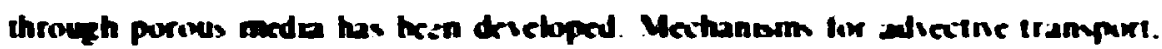

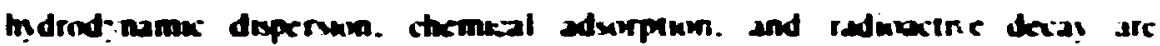
meluct in the mathematical inmmubion !mplementatmm of youdribicral tins":

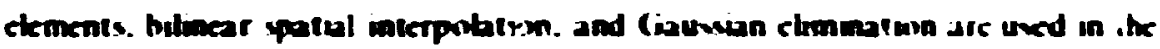

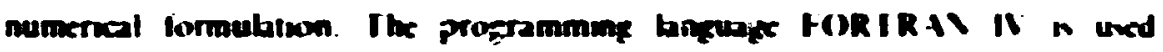

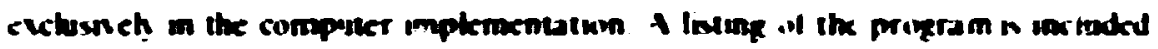

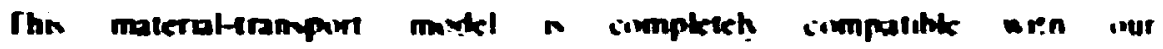

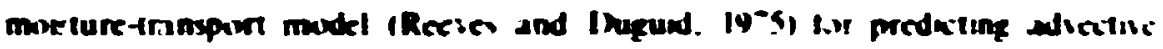
Darev velucrtics for poridus mada a huch mas he parth unvlurated

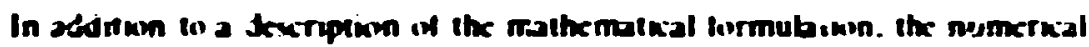
treatment and the computer implementatmon revults if ton cermputer umulatum

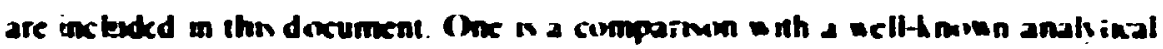
treatment (1 apides and imundwm. 19521 and is micnded as a partul valdatum

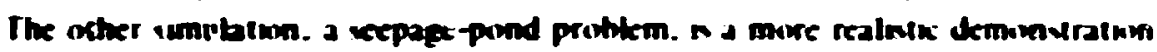

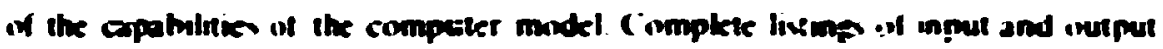

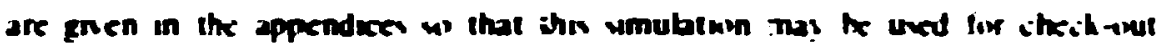

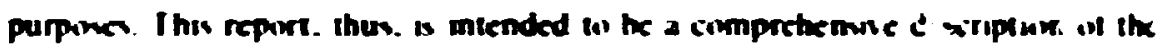
materul-trimpint computet makel
\end{abstract}




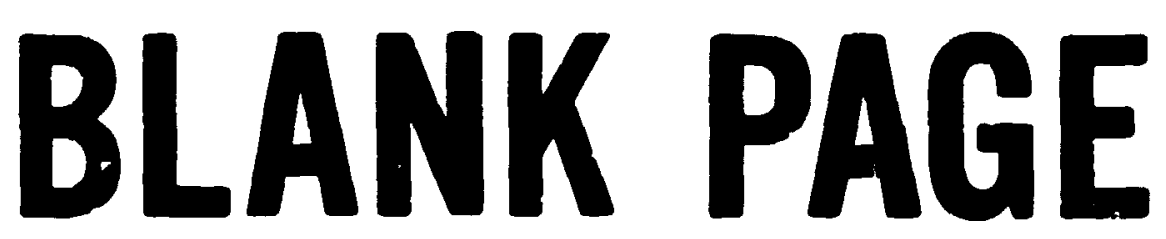

(2) 


\section{INTRODUCTION}

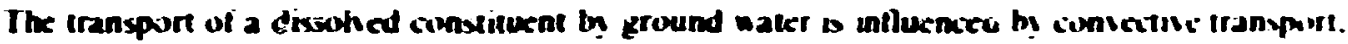

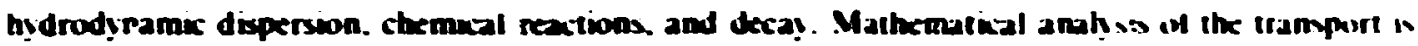
further compleaked by transient multidimenswoal flow of the carrer. whin ina! initur under

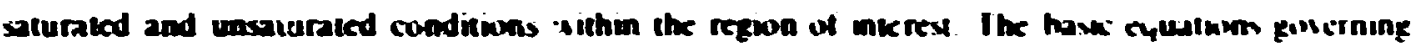
the Iransport have been tormubted for various ptnswal and chemual properties m mun!

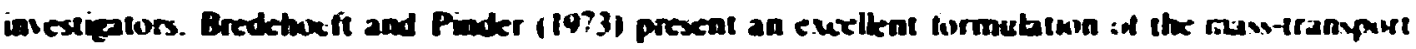

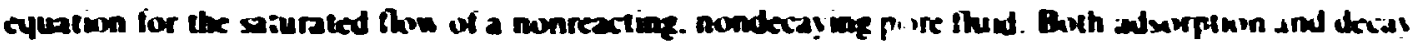
of the disched constituen are considered in the eyuatiuns for saturated iranspurt whnt: ats:

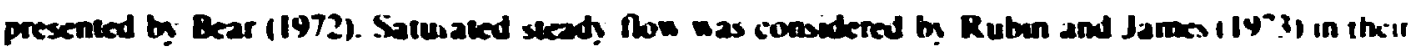

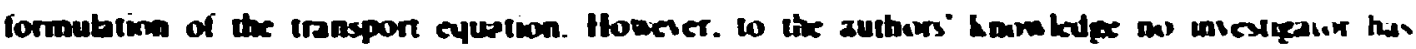

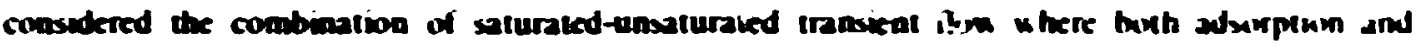

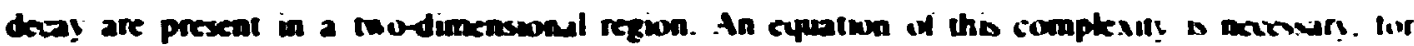

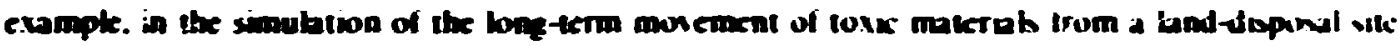

Mathernatical desciption of chemical reactwos that oxcur when diswhed comsinuents flow through porous media may be quite detailed. Both reversible and irreversible chemucal reatmms mis!

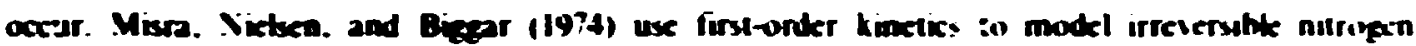

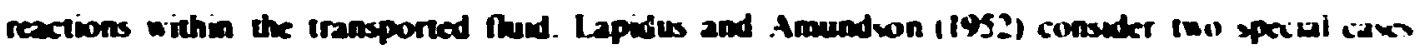

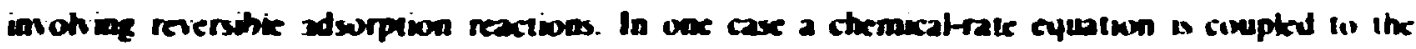
trampon eypation. in the cther case a bealeyuiliteram assumption is made in which the solid-phase concentration is a simple lear function of the liqud-phaxe concentratum and is independent of tine. Suxh a iunction. cal'xd an atsorption sotherm. ma! be nonilencar il al ans Jurinak. 1972) and hysteretic as applied by Van fenuchien. Lasidson: and Wierenga (197t). Ihe

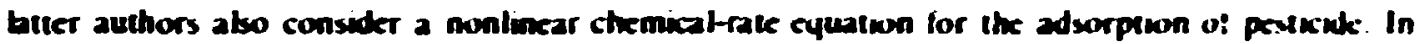
adutition to the various aspects of reversible and irreversuble reactions. these mag be seteral specikes of dissotied constituents in the sobution either reacting ureversibh wh weach other or competing tor araibble adsoption sites in the soil matrix (Rubin and James. (973).

The chenising considered in this document is moteneral enough to encompass all of the complexities given in the previous paragraph. The rescting species in the soli sobution are assumed (f) be completely independent so that they may be treaked one at a ume $m$ the computer cuxte for reversuble proceses bcal cyuilibrium is assumed and a linear isotherm function is cmphyed. Finalis. the only irreversible reaction that may be considered is one analnews to radinactive decas: i.e. Ih: rale of uncrease for decreasel is proponional to the imal quantit! of the constituent prewent in buth the solid and the linjid phases. Th.se restictions are in agreement with the application. currently contemptated by the authors.

Solution of the iranspor: equation may be ohtained by anatytcal means under very seicre resirictions for onedimensional fho Ilapidus and Amundson. 1952). However. Inr more maningful application of the eyuations in rezions which approximate conditions that iccur in nature. numerical methods of solution are reyuired. Several invesigators have emplọed finite-difference methods for the solution of the convective diffusion eyuation (Oxte:. et al.. 1970) and of the iransport cyution where adsorption was considered (I ai and Jurinak. 197I and 1972). Hiwever. as pointed out by Buedrhneft (1971). these methods produce numerical dispersion of the 


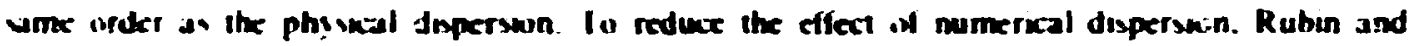

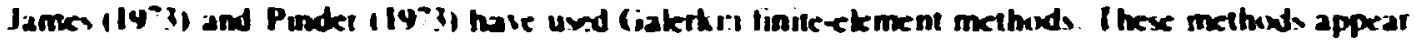

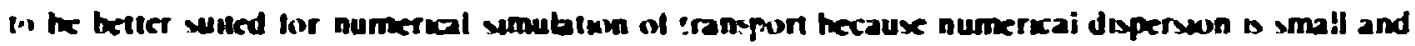

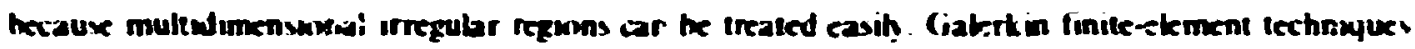
are emplised by the autuon in a companion cocument (Reesss and Dugud. 19?5). which ireats inv-dimenswal water how through viturated-umaturated porous media.

Ihe purponc of detehpong a two-dimensonal tramient model in wheh both adsorptwn : nd

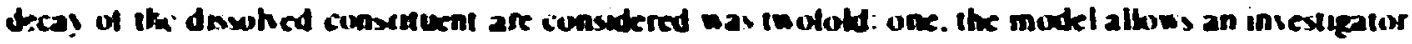
Io vmuble he traspent of tosx makerab throngit seuraked-unsaturated porous media to predict tuture conceniratums in the ground acter: and. two. the model pronute the coxic-material coucentralion data necesong hi human-dose cakubtions. 


\section{Il. THEORETICAL DEVELOPMERTT}

In the following sections the equations governing mass transport throngh porous media are formulated and the correspending Gakrkin fixite-element soluison techniques zre developed. In the following formulation the fluid phase carries a dissolved constituent which is adsorbed by the solid. Thus. the constituent is present in both the fiuid and the solid phases of the medium. The concentration of the dissolved constituent is assumed to be suffeciently small that c.anges in concentration do not affect transpen of the fluid. The dissulved constituent in the cartier is assumed te be in equilibrium with the concentration of the constituent adsorbed by the solid, and the rate of decay is assured to be proportional to the concentration.

1. Formulation of the Mass-Trassport Equations. Consink- an arbitrary material volume element of saturated-unsaturated porous mediz. The volume ekment is composed of fuid and solid. beth of which are moving. Since the velocity of the solid phase is smalter than the velocity of the flud. it is convenient to attach the coordinate system to the solid. This requires that no solid grains eross the surface of the volume element as it deforms. The material volut ie element moves through space with a velocity $\vec{v}$., while the fluid phase flows through the surface with a bulk flux $\vec{f}$. Thus. the term $\vec{f}$ is the bulk flux of the fluid relative to tixe solid. The total amount of the constituent within a volume $V$ at any instant of time is

$$
\int_{v}\left(\theta c+p s^{\prime}\right) d V
$$

where $\theta$ is the water content, $c$ is the concentration of the dissolved constituent in the water. $\rho$ is the bulk density of the solid, and s' is the concentration of the constituent that is adsorbed by the solid. The rate of mass transport through the surface of the volume is

$$
\oint_{s} \dot{n} \cdot \vec{f} \text { as }
$$

where $\boldsymbol{n}$ is an outward-directed unit normal to the surfac:. The rate of change of the amount of the constituent in the volume due to decay is

$$
\lambda \int_{v}(\theta c+\rho ;) d v
$$

where $\lambda$ is the decay constant.

From Eys. (1-3) conserva on of mass may be written as

$$
\frac{d}{d t} \int_{V}\left(\theta c+\rho s^{\prime}\right) d V+\int_{S} n \cdot\left(d S+\lambda \int_{V}\left(\theta c+\rho s^{\prime}\right) d V=0\right.
$$


where $d$ d: is the material time derivative

$$
\frac{d}{d t}=\left(\frac{\partial}{\partial t}+\bar{v}_{s} \cdot \eta\right)
$$

When Reyiolds transport theorem (Maliern. 1969. p. 210-211) is applied to the firs: term of Ey. (4). the following equation is obtained:

$$
\int_{v} ! \frac{\partial}{\partial t}\left(\theta c+\rho s^{\prime}\right)+\vartheta \cdot\left(\theta c+\rho s^{\prime}\right) \bar{v}_{s} ! d V+\int_{s} \bar{n} \cdot \bar{f} d S+\lambda \int_{V}\left(\theta c+\rho s^{\prime}\right) d V=0
$$

The divergence theorem is

$$
\oint_{S} \vec{n} \cdot \vec{f} d S=\int_{V} v \cdot \vec{i} d V
$$

When Eq. (?) is combined with Fy. (6). the resulting equation is

$$
\int_{v}\left|\frac{\partial}{\partial t}\left(\theta c+\rho s^{\prime}\right)+v \cdot\left(\theta c+\rho s^{\prime}\right) v_{s}+\nabla \cdot \bar{f}+\lambda\left(\theta c+\rho s^{\prime}\right)\right| d V=c
$$

Since the integrand, in general. must be zero. conservation of mass becomes

$$
\frac{\partial}{\partial t}\left(\theta c+\rho s^{\prime}\right)+\eta \cdot\left(\theta c+\rho s^{\prime}\right) \bar{v}_{s}+\nabla \cdot \bar{f}+\lambda\left(\theta c+\rho s^{\prime}\right)=0
$$

The bulk flux of the constituent and carrier relative to the solid may be divided into a diffusive flux and an advective flux:

$$
\bar{f}=\bar{f}_{\mathbf{J}}+\overline{\mathbf{f}}_{\mathbf{J}}
$$

When a concentration gradient within the individual pores is assumed. the diffusive flux is written as

$$
\bar{f}_{d}=\theta \overline{\bar{D}} \cdot v_{c}
$$

where $\overline{\bar{D}}$ is the hydrodynamic dispersion tensor. The advective fux of the constituent and carrier may be written in terns of the flux of the carrier as 


$$
\bar{f}_{s}=s \bar{v}_{i_{V}}=i \bar{v}
$$

where $\vec{V}$, is the velocity of the carrier relative to the solid and $\theta \mathrm{V},=V$ is the Darey Mux. Substitution of Eqs. (10-12) into E4. (9) yiclds

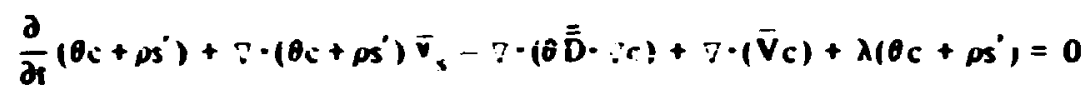

The second term of Ey. (13) may be expressed as

$$
\Gamma \cdot\left(\theta_{c}+\rho s^{\prime}\right) \bar{v}_{v}=-\left(\theta c+\rho s^{\prime}\right) \cdot \bar{v}_{s}+\left(\theta c+\rho s^{\prime}\right) Z \cdot \bar{v}_{s}
$$

where the first term on the right-hand side of the eyuation is the product of two small tectors and will be neglected. If all of the displacement of :he medium is assumed to he vertical (e.g.. vertical consolidation). the last term of Eq. (14) becomes

$$
\because \cdot \bar{v}_{v}=\frac{\partial e}{\partial t}=\alpha \rho_{i} g \frac{\partial h}{\partial t}=a^{\cdot} \frac{\partial h}{\partial t}
$$

where $c=e_{i n}$ is the dilatation of the medium. $a$ is the coxfficient of compressibility of the medium. $\rho_{r}$ is the density of the nuid. $z$ is the acceleration of gravity. $h$ is the nuid pressure head. and $a^{\prime}$ is the modified coefficient of compressihility of the medium. $A$ more detailed formulation of Eq. (15) is given by Keeves and Duguid (1975). Substitution of Eqs. (14) and (15) into Fq. (13) yields

$$
\frac{\partial}{\partial t}\left(\theta c+\rho s^{\prime}\right)+\left(\theta c+\rho s^{\prime}\right) a^{\prime} \frac{\partial h}{\partial t}-\eta \cdot(0 \overline{\bar{D}} \cdot 7 c)+\eta \cdot\left(\vec{V}_{c}\right)+\lambda\left(\theta c+\rho s^{\prime}\right)=0
$$

The adsorption of the constituent by the solid is assumed to occur at a rapid rate (i.e.. a fast exchange reaction) such that the dissolved material in the carrier is in equilibrium with the material adworbed by the solid. This is expressed by the linear equation

$$
s^{\prime}=k
$$

where $k$ is the distribution coefficient for unsaturated pornus media. For unsaturated media, $k$ is defined as 


$$
k=\frac{\text { quantity of adsuntied material:mass of sevlid }}{\text { quantity of dissulved material vulume of fluid }}
$$

where the volume of nuid is eyual to the moisiure content times the total velume. For saturated medin.

$$
k_{d}=\frac{\text { quantity of adworbed materialimass of suld }}{\text { quantily of dissulved material/rulume of liud }}
$$

where the volume of the fluid is equal to the sorosity times the total volume. Thus. the relatioiship between the unsaturated and saturated distribution coefficients may be written as

$$
k=\frac{\theta}{n} k_{d}
$$

and the material adsorbed by the solid is expressed as

$$
s^{\prime}=\frac{\theta}{n} k_{d}
$$

Substitution of Eq. (21) into Eq. (16) ! iclds

$$
\frac{\partial}{d t}\left(\cdot+\frac{\rho k_{i}}{n} \theta_{c}\right)+\left(\theta_{c}+\frac{\rho k_{d}}{n} \theta_{i}\right) \cdot \frac{\partial h}{\partial t} \quad \nabla \cdot(\theta \overline{\bar{D}} \cdot \nabla c)+v \cdot\left(\bar{V}_{c}\right)+\lambda\left(\theta_{c}+\frac{\rho k_{d}}{n} \theta_{c}\right)=0
$$

The retardation factor is defined as

$$
R_{d}=\left(1+\frac{\rho k_{d}}{n}\right)
$$

This term is teasure of the delay or retardation of the breakthrough of the dissolvec constituent. Substitution of the retardation factor into Eq. (22) yields

$$
R_{d} \frac{\partial\left(\theta_{i}\right)}{\partial t} \quad \nabla \cdot\left(\theta \overline{\bar{D}} \cdot \nabla_{i}\right)+V \cdot\left(\nabla_{c}\right)+\left(a^{\prime} R_{d} \frac{\partial h}{\partial t}+\lambda R_{d}\right) \theta_{i}=0
$$

which may be expressed in the form 


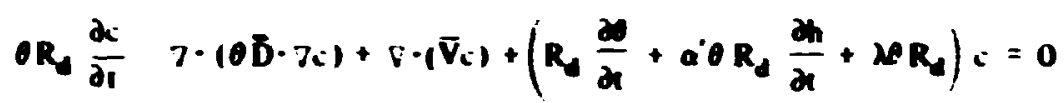

In a porous medium which is assumed to be isctropic "ith reypect to dispersivity. the hydrodyname dispersion coefficient may be written (Bear. 1972) as

$$
\theta D_{i j}=a_{T} V^{\prime} \delta_{i j}+\left(a_{L} \quad a_{T}\right) \frac{V_{1} V_{i}}{V^{0}+a_{i j}} \tau^{i} \delta_{i j}
$$

where double indices denote stimmation. 6 . is the Kronecker delta. $a_{1}$ is the transierse dispersivity. $a_{1}$ is the longitucinal dispersivity. $a_{x}$ is the molecubr diffusion. $z^{-}$is the tortuosity. $V^{\prime}$ is the magnitude of the Darcy velocity. and $V$; and $V$ are the components of the Darcy veiocity.

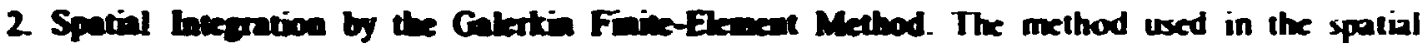
integration of Ey. (25) is a special case of the broad calegory called weighted-residual methods. These methods. in their classical form. approximate the solution to the differential eyuations and satisfy the boundany conditions exacth. The differ:a ial equation may be written in the general form

$$
L \Phi)=0
$$

where $L$ is a differential operator and $\mathcal{f}$ is the dependent viable. The weighted-residual approximate suiution $\phi^{\prime}$ over the region $F$ is obsained from the equation

$$
\int w_{j} u \phi^{\prime} x v=0
$$

The $W$.omprise a set of $p$ weighting functions. and $\phi^{\prime}$. the trial solution. has the form

$$
\phi^{\prime}=\sum_{1}^{m} \gamma_{1} o_{1}
$$

where the $X$, are trial functions, the $\$$ are unknown amplitudes of the irial solutions. and $(.1 \$) \neq 0$ is the residual. The special case in which the weightirg functions $W_{\text {; }}$ are chosen as the trial functions $X$ is the Gakerkin method. Ir. effect. the equations for the Gakerkin method

$$
\int_{v} x_{1} L\left(\sum_{1}^{m} x_{1} \phi_{1}\right) d V=0 \quad J=1111 x
$$




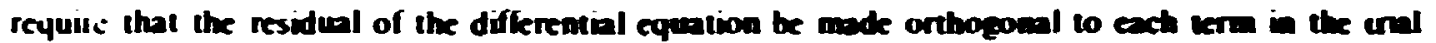

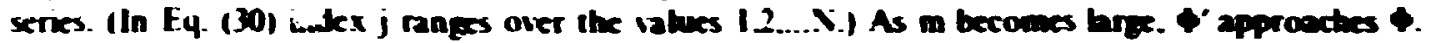
Cuefficients o may be obtained by ineegation and solumion of the above equations, and the approximate solution $\phi$ is obtaned by substituting thes coefixients into the trial solenion summation (fintasion. 19?2).

In the rissical Gakerkin method. each of the trial sohutions $X$. excends over the catire bomain I and must sativiy all boundary conditions. Hence. the method is restricted to simphy shaped. sisoply connected regions with homogencous material properies. However. the power and generalixy of the Cakerkin method can be exkended considerabh by combining it with a faike-element discretiantion in which the region of integration is represented on an assemblape of subdamaims. In two-diresiciomal space these elements would be polyeons. and in inre-dinemsional space they would be poht hedra. These subdomains are called elemeats, and their corners. or connection points. are calkd nodes. In this approach. the famihy of trial solutions consists of subfamilies of very simple functions. The $\mathcal{C}$ sa:isfy the boundany conditions. and not the besis functions $\mathrm{X}$. which are nonneto on only onk of the subdomains. The coeffecients becosne the amplinudes of the ualnown function at the nodes. This forite element approach. in effect. is a piecenise Calertin approxianation which permits the application of the Calerkin method to complex ecometries and noabonogeneoun media. A more detailed diccussion of the Calertion finite element method is given by Hutton and Anderson 19?1). The formubiion and use of the finite element Gaktsin method in ground wacet transport is presented by Pinder (1973).

In such a formubtion in is coavenient to introduce one basis function $\{\mathrm{N}(\bar{x})$; for eacti element $r$. This combination weiphting-frial fuaction is. however. a column vector that has a separate $N(\bar{x})$ for each node $i$ of the element. Each of these bilinear interpobtion functions extends actoss the entire element in a two-dimensional space. Each has a mapanude of uaity at node $i$ and a magnitude of tero at all other nodes. Because of the beter property. coefficients c. of the trial solution

$$
p=, 11 \times 11=4 \times 1)^{1}+111
$$

are identical to the concemtration at each node as anticipated by the new notation for expansion of the coeffecients o on the righthand side of Eq. (31). When Coktkin's method is applied 0 Eq. (25) in the $r$-th element. the equations are

$$
\int_{v} d N \cdot\left|A^{-} t+B^{-}\right| d \mid d V=0
$$

where $\dot{c}=d c$ di and

$$
\begin{gathered}
A^{*}=R_{d} \\
B^{-}=\because \cdot 106 \cdot \because 1+\because \cdot c+\left(R_{d} \frac{\partial}{\partial} \cdot 0 R_{d} \frac{\partial h}{\partial} \cdot 2 R_{d}\right)
\end{gathered}
$$

Equation (31) combined with Eq. (32) gives

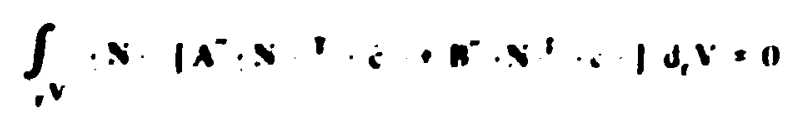


where the notation $f . c_{i}^{\prime}$ thas been changed to $f c f$. Eywation (34) man be simplified to

$$
\int_{, v}||,+1|\cdot|, B \mid \cdot 1,1,=0
$$

(i3)

where

$$
\left|A^{\prime}\right|=\cdot x \cdot A^{-} \cdot x \cdot 1
$$

and

$$
\begin{aligned}
& \mid, B I=: N: N=N \cdot N=\frac{\partial}{\partial x_{1}} \theta D_{1} \frac{\partial}{\partial x_{1}}+\frac{\partial}{\partial x_{1}} v_{z} \\
& \left.+R_{d} \frac{\partial \theta}{\partial i}+a \theta R_{d} \frac{\partial h}{\partial t}+\lambda \theta R_{d}\right): N: T
\end{aligned}
$$

When Green's theorem is applied to the integral over the element bolume of the first two kerms of Ey. (37), it follows that

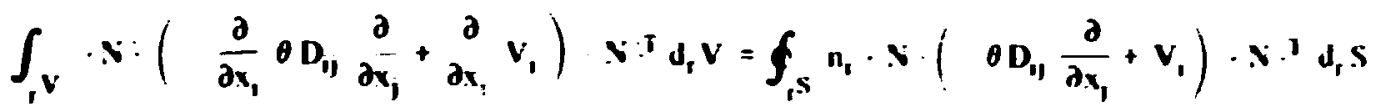

$$
\begin{aligned}
& \int_{r} \frac{\partial}{\partial x_{1}}: N:\left(\theta D_{11} \frac{\partial}{\partial x_{1}}+V_{1}\right) \cdot N \cdot T d v
\end{aligned}
$$

and Eq. (35) may be written as

$$
|, A|: C+|, B| \cdots+\because, Q:=8
$$

where

$$
\mid, A\}=\int_{, v}\left(N:\left(R_{d} \theta\right): N ; d_{,} V\right.
$$




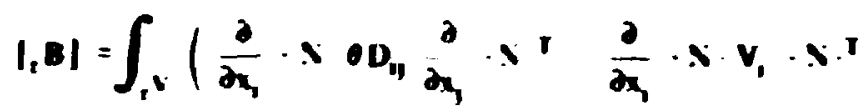

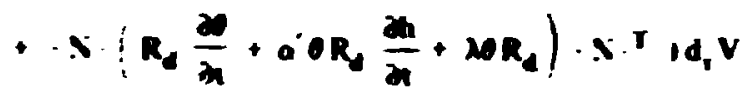

and

$$
\cdot 0:=f_{r} n_{1} \cdot x\left(\theta D_{1} \frac{\partial}{\partial x_{1}}+v_{1}\right) \cdot x \cdot \cdots d_{1} s
$$

The tern in square brackets in Eq. (42) is defined by Eqs. (10-12) and mas be expressed as

$$
\therefore:=\int_{s} \cdot \Omega \cdot n \cdot f d_{r} s
$$

Thus. Eq. (43) expresses the bulk now of the dissolted constiucent across the boundan of the ekment.

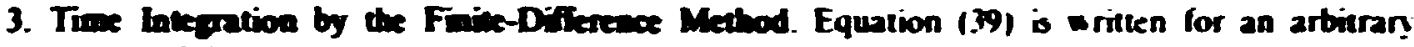
increment of time $\mathrm{It}$ :

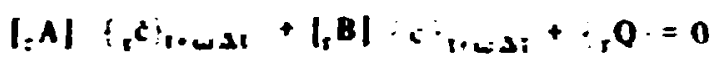

is the Crank-Nicholson centered-intime approach $\omega=12$. and in the beckward-difference

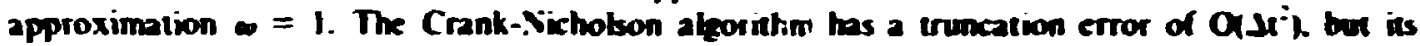
propagation-oferror chapcteristics frequenth lead to oscillatory iastabilines. The backward difference scheme. On the other hand. has a (runcation error of $Q 13$ ) bu is quile resistant to excillatory instabilities. An arbitrary allows an investipater to find the appropriake babace for the problem being considered. The time derivative of the concentration is expresed as

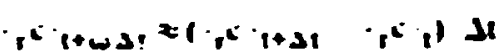

and the value of this quantity at an arbitrary point in tinte is taken as

$$
r c \cdot 1+\omega d t=w \cdot r \cdot 1+31+(1 \quad \omega) \cdot, c \cdot 1
$$


Substitutinn of Ey. (45) and (46) into Ey. (44) yields the follow ing rebtiunshep:

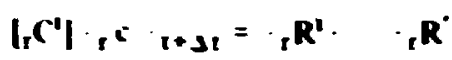

where

$$
\left|C^{-1}\right|=\left|A^{\prime}\right| \mathbf{I}+\omega|r|
$$

and

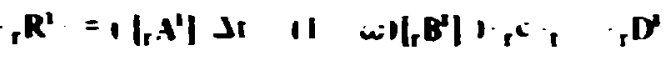

It should be understond that matrix, $[A]$ and $[. B]$. and. hence. [C] and,$R$ ! . are evaluated at tume $1+1$.

4. Nancial lyphemation. For a yuadribleral ekment wh four corner modes. a balincar polynomal basis function for the $j$ th node may be a ritken in kerms of hnal normalined courdinates as

$$
v=\frac{1}{f} 11 \cdot 3,11+\pi_{1} \quad 1=1114
$$

where $s$ and $t$ are the bocal coordinales of the corner nodes, which are numbered in 4 progessung around the element in a counkerclockwise direction (fig. I). In the bocal courdinate sysuem the element is upare repardless of the shape of the quadribieral in global comorduates. The dohal coondinates at any point within eleanent 1 are given in kerms of boal cuordianales by the relations

$$
\begin{aligned}
& x=x^{\top}, x: \\
& r^{\prime}=\cdot \lambda \cdot r^{\prime}
\end{aligned}
$$

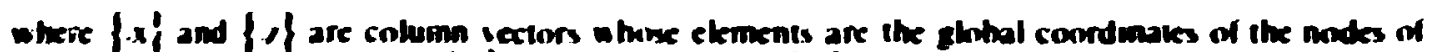

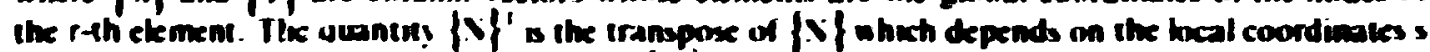

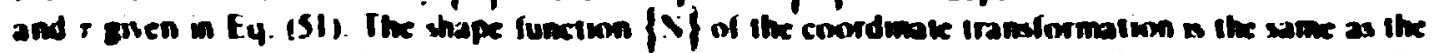

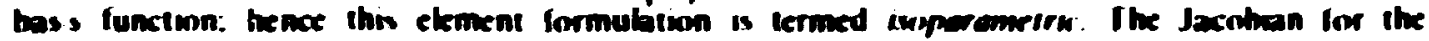

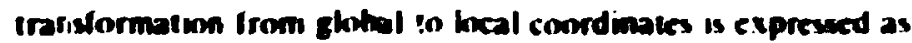

$$
|r|=\left[\begin{array}{ll}
\frac{\partial_{1} x}{\partial s} & \frac{\partial_{1} \prime}{\partial_{s}} \\
\frac{\partial_{2} x}{\partial \tau} & \frac{\partial_{1} \prime}{\partial r}
\end{array} \mid\right.
$$



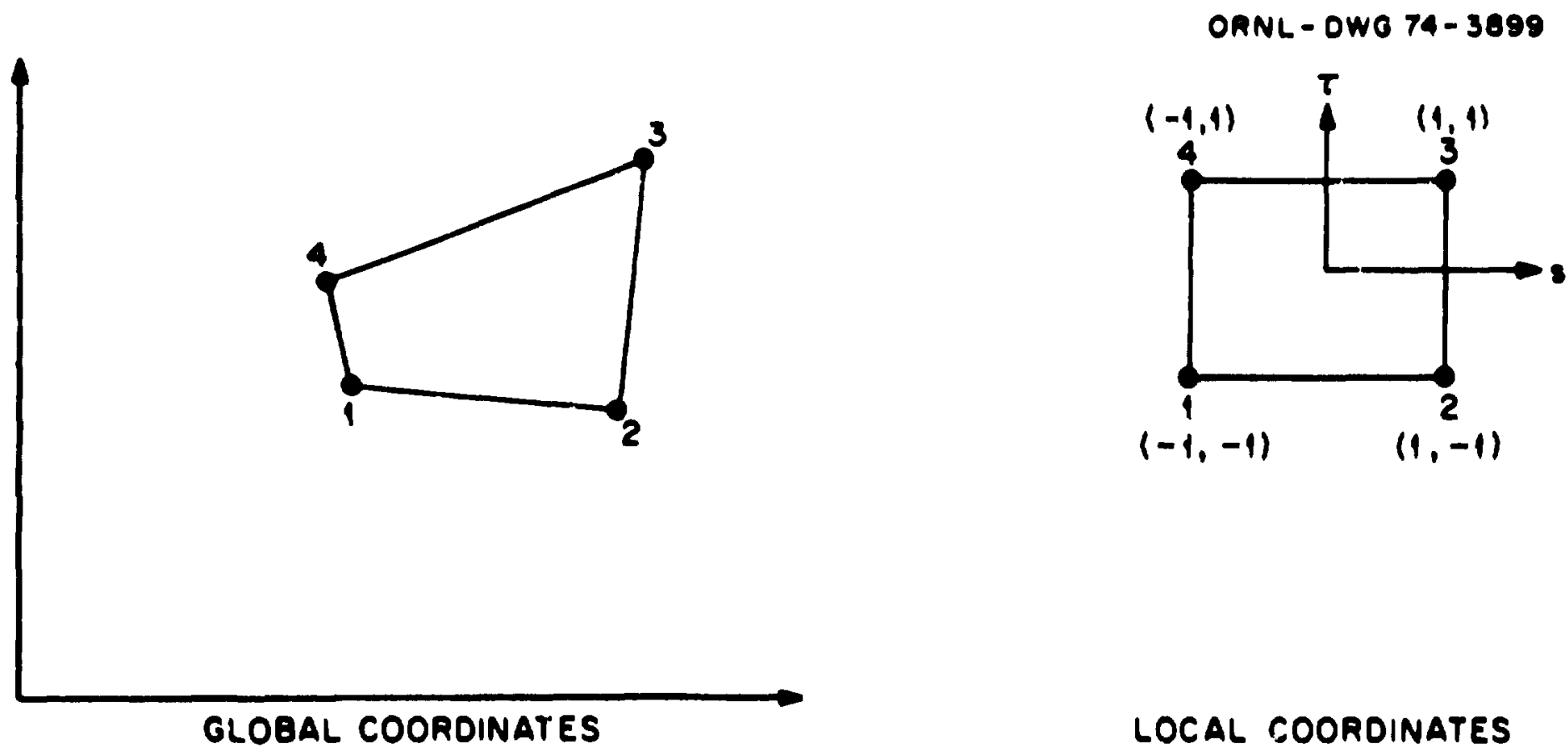

$=$

Fig. 1. The rath Finite Element in (ilobal and local Coordinates. 
Substitution of Eq. (51) iato the deverwionat of this expresswon selds

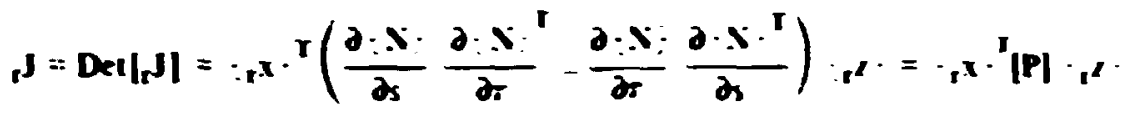

where $[P]$ is defined as

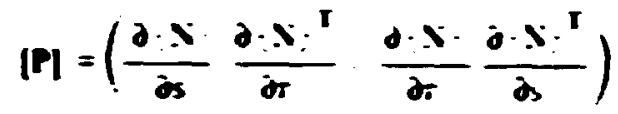

Whea the expression for $\{\mathrm{X}\}$. Ey. (50). is used. in may be shown that

$$
|P|=\left[\begin{array}{ccccc}
0 & 1 & 1 & -s+1 & 1+x \\
1+ & 0 & 1+s & s= \\
s= & 1 & 1 & 0 & 0
\end{array}\right]
$$

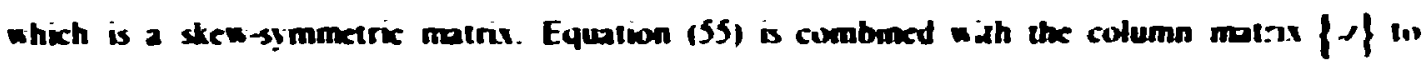
sichd

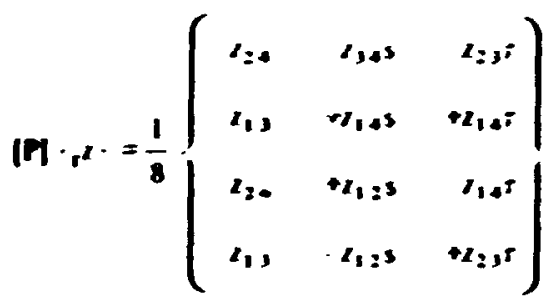

and the determinant of the Jazoban is

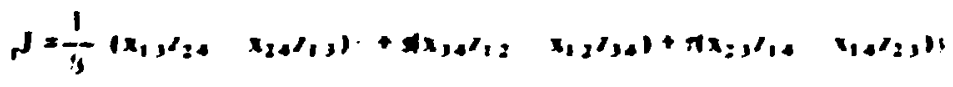

Terms $x_{i}$ and $t_{r}$ are defined $a$.

$$
\begin{aligned}
& x_{4}=x_{1} \quad 8 x_{1} \\
& \text { 10.4 }
\end{aligned}
$$




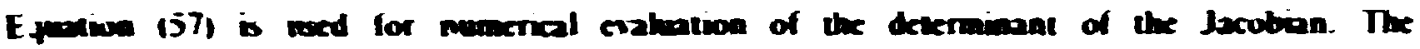

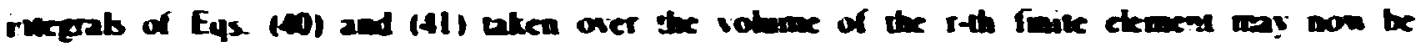

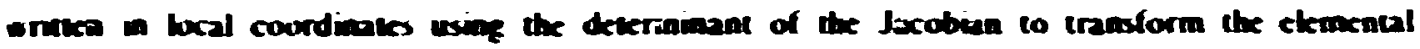
area:

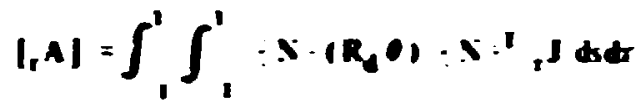

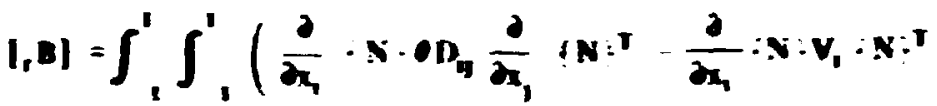

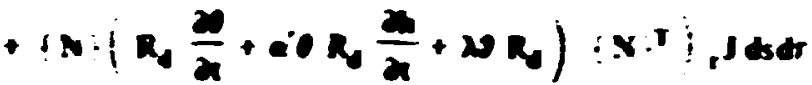

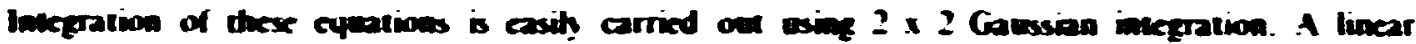

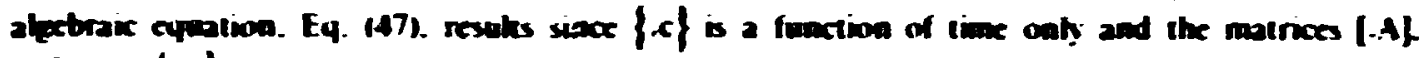
[B] . and \{R\} are crainaked for the previons time step.

Ia order to evaluate [A]. Eq (60). expressions for the spatiol detivative of the inerpobtion functiona are necessa:

nang be inerted lo yietd

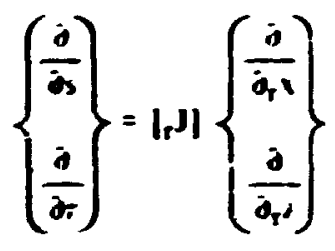

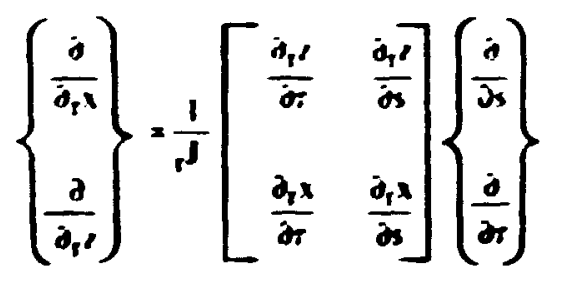

mine the defmition of (J). Eq. (52). When the top row of Eq. (62) is applied to the basis fanction $\{\mathrm{X}\}$. the followine is diained:

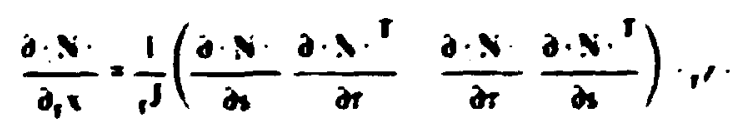




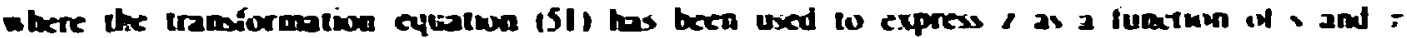

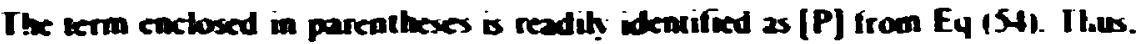

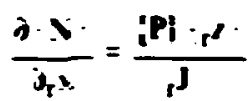

in ar. caturety analoguts way it may abo be shown that

$$
\frac{\partial \cdot X \cdot}{\partial_{\mathrm{r}} \prime}=\frac{-[P] \cdot{ }_{\mathrm{r}} \mathrm{x}}{\mathrm{r}^{J}}
$$

Equations $(64)$ and $(65)$ are in a form suitable for numerical eraluation. These cyuations and their trasposed counterparts are used to enaluate the iniegraad of [.B] . EY. (60).

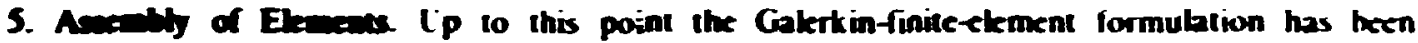
presened for the $r$ th element in the collection of elements which comprix a region. Within this formulation only coupliag betwicen the aodes of the rth eleurent has been consideted. However. in the region a node may be coupled in the several elements in which it is contained. The assernby process. which is a special type of summation. imbeds eich element matrix in a braer global matrix such that the appropriate coupling is established. In order to understand the ascembly of the elements that form a system of algebraix eyuations. a simple example will be wed. The exampte selected is that of a two-dimensional space that is disided into twehe rectangular elements (fig. 2). Iteration superseripts are dropped for convenience Both dobal and local subscripting of the $\left[{ }_{i} C\right]$ matrix are shown in Fig. (2b). Expansion of matrix [ ${ }_{1} C$ ] into a composite-matrix form is shown in Fig. 3. Assembly consisis of summing over the expanded form of each [C] to form the composite matrix [C]. The compiete [C] matrix will be sparse and banded. The band width may be cakulated from the equation

$$
\text { IBAND=24MAXDIFHI }
$$

where MAXDIF is the maximum nodal difference in any element of the system and IB.ANI) is the band width. Thus. for the example problem the hand width is 11 . For more ceonomical use of compulet storage. only the band portion need be stored. The most economical form of storage of the banded matrix is shown in: the lower porion of Figure 3. A more detailed discussion of the assembly of finite elements is presented by Desai and Abel (1972).

At this poini it is interesting io note that the band width is controlked by the glohal modal numbering system. A reduction in computer core storage is achieved by reducing the magnitude of the term MAXDIF. This reduction is oblained by numbering in the direction in which there are the least nodes in a given row or column. In the example, if the nodes were numbered in a horizontal direction. the band width would he 13. This represents a significant increase in the core storage required. 


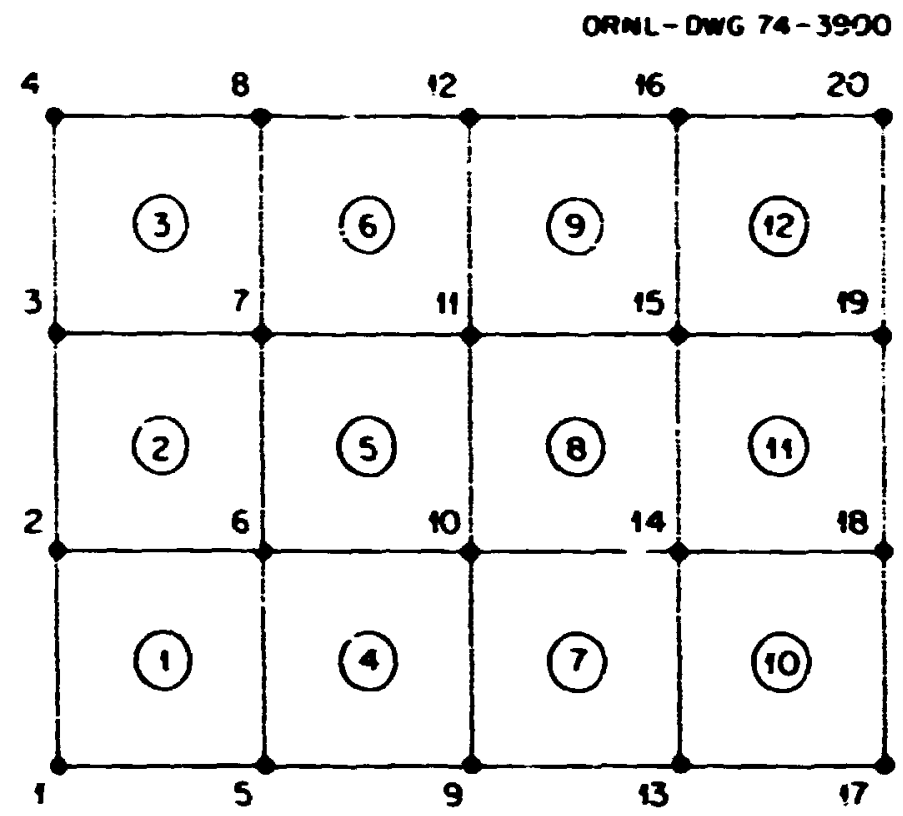

(a)

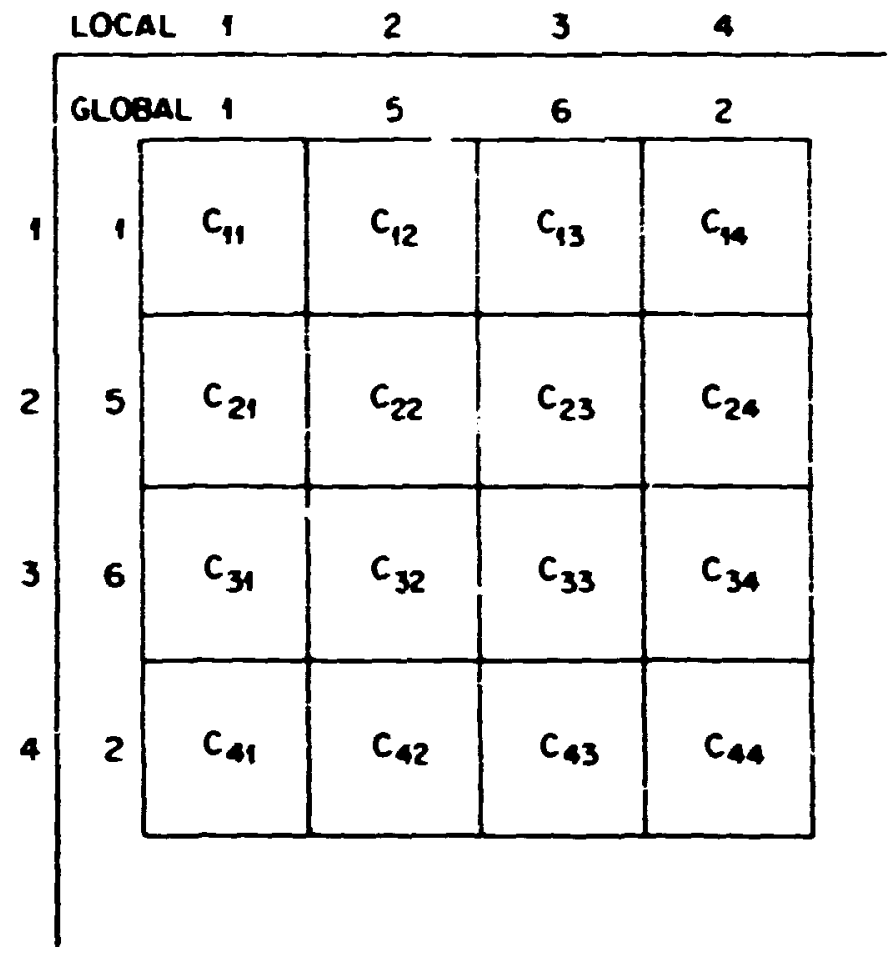

(b)

Fig. 2. Example Problem. (a) Element representation of the space using slobal numbering system. (b) Local and global numbering schemes for ekment matrix [,C]. 

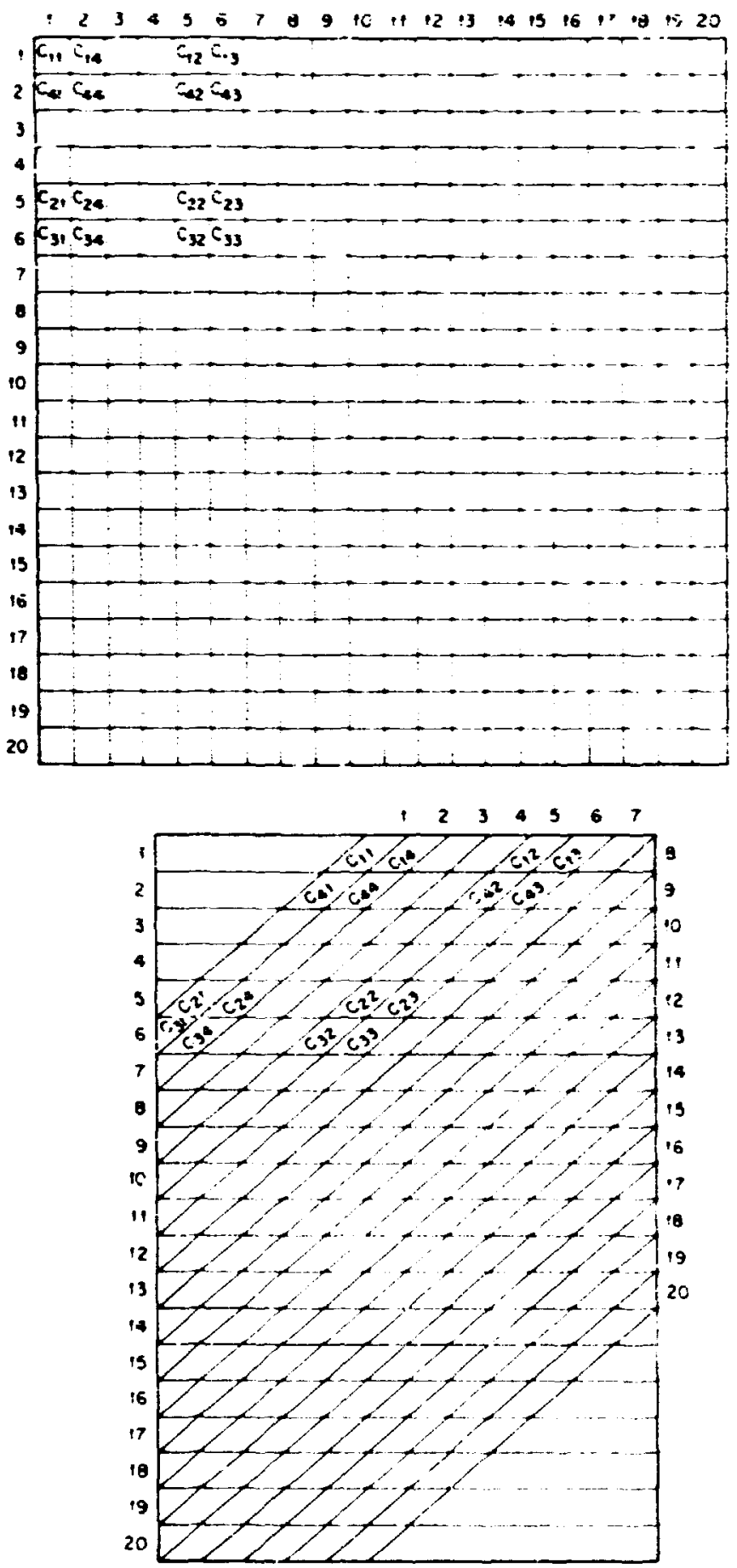

Fig. 3. Complete and Banded Matrix [C] for the Example Problem. 
Eyuation (47) is evaluated and assembled for each element. and the assembled system of algebraic equations may be written as

$|C|\{\}_{1+\Delta 1}=\{R\}-\{Q\}=\{Y\}$

providing $\{Q\}$ is not a func ion of the concentrations.

6. Application of Doundary Comditions. Surface. on which Neumann constant-flux conditions are impused. of course. yield concentration-irdepend:nt entries in the column matrix $\{Q\}$. These entries are evaluated by direct application of Eq. (43) to give normal flux vectors $\bar{f}$ followed by assembiy over all boundary elements whose sides have constant-flux conditions. The results are then subtracted from $\{R\}$ to form $\{Y\}$ as shown in Eq. (67).

Vieumann variable-flux conditions are employed. however. to simulate seepage surfaces. The corresponding entries in $\{Q\}$ are then linear functions oi the unknown concentrations. Such terms must therefore be incorporated into the [C] matrix in Eq. (67). Advection. Ey. (12). is assumed to be the dominant iransport nechanism at the seepage surface. In this case Ey. (43) becomes

$$
\{, Q\}=\int_{r}\{N\} \pi \cdot c \vec{V} d_{r} S
$$

where the surface integral covers only those sides of element $r$ which hound the entire system. (Integrals over internal element surfaces add to zero during assembly and need not be considered.) Expanding $c$ using Ey. (3I) yields

$$
\{,\{\}=\{, E \mid\{, c\}
$$

wh.

$$
\mid, E\}=\int_{r}\{N\} n \cdot \dot{V}\{N\}^{T} d_{r} S
$$

Application of the time-ink.gration algorithm. Eq. (46). yields

$$
\left.\left.\{, Q\}=\omega \mid, E\} ', i I_{1+\Delta 1}+\left(\begin{array}{ll}
1 & \omega
\end{array}\right) I_{r} E\right\} I_{r} c\right\}_{1}
$$


The term o[,E]. after assembly over all boundary elements $r$. is added to matrix [C] . whereas the term $\{1-w h, E \mid\{, c\}$. after a similar assembly process, is subtracted from $\{R\}$. A matrix cyuation having the same form as Ey. (67) resuls.

At nodes where Dirichkt (constant) boundary conditions are encountered. an identity eyuation is generated for each such node and included in the matrices of E4. (67). As in example. take a oneelement system with the concentration at node I constrained to the value of $b$ at all times. i.e..

$$
c_{1}=b \quad \text { and } \quad b \neq b(t)
$$

Equation (67) then takes the form

$$
\left[\begin{array}{llll}
I & 0 & 0 & 0 \\
0 & C_{2:} & C_{23} & C_{24} \\
0 & C_{32} & C_{33} & C_{34} \\
0 & C_{42} & C_{43} & C_{4+}
\end{array}\right\}\left\{\begin{array}{l}
c_{1} \\
c_{Z} \\
c_{3} \\
c_{4}
\end{array}\right\}=\left\{\begin{array}{ll}
b & C_{2, b} \\
Y_{3} & C_{3, b} \\
Y_{4} & C_{41} b
\end{array}\right\}
$$

This result may easily be generalized to an arbitrary number of equations with an arbitrary number of Dirichlet boundary nodes.

7. Solution of the Assembled Equations. In solving the assembled equations exprexsed in Fy. (67). the matrix [C] is decomposed into the product of upper and lower irianguliar matrices using the fauss technique. The lower triangular matrix is used to modify the right-hand side \{Y\} for back-substitution into the upper triangular matrix to obtain a solution. If the matrix [C] and the time step Jt do not change with tiine, the decomposicion needs to be periormed only once. 


\section{COMPUTER IMPLEMENTATION}

The computer program consists of the It different subprograms shown in Fie. 4. As is inplied by its centra! location. the routine MAIN petforms the control function for the program. i.: it. input-output operations are coordirated with primagy and support coaputations as the calculation is stepped sequentially over the time variable.

Figure 5 is a block dagram showing how the primary computations are ordered. First. moistiretransport variables. pressure H. moisture content TH. and Darey huxes VX and VZ are input if these quantitics have time variations: and tise derivatives of $H$ and $T H$ are determined as required by the iransport Eq- (25). Then. using parameters characteristic of both the dissolved constituent and the soil types. element integrab are evaluated in subroutine $\mathbf{Q 4}$ in the following manner: Each quadribteral element is tranoformed to a local coordinate system where it becomes a square with a side kngth of 2. Iacobians [Eq- (53)]. Gakrtin basis functions [Eq. (S0)]. and derivatives of these functions [Eqs. (64) and (65)] are determined: and a $2 \times 2$ Gauss quadrature is employed to yield elewent matrices [.A]. Eq. (59). and [,B]. Eq(60). In subroutine ASEMBL. these matrices are combined in accordance with the lime-integration algorithm. Eys. (\$8) and (49). to form matrix $[C]$ and vector $\{, R\}$. subscripts are adjusted. and $a$ sum over all elements $r$ is effected as required by the assembly process (see (hapter II. Section 5). Since the assembled matrix [C] is asymmetric. its entire band must be stored.

Vext. using routine BC. boundary corditions are incorporated into the systems equations. E4. (67). Teumann constant-flux conditions require only a change in the load vector (Y\}. For veumann variabie-flux (seepage) and Dirichkt boundary conditions. however. it is necessany to modify both coefficient matrix [C] and load vestor $\{Y\}$ as discussed in Chapter II. Section 6. Boundary integrals [.E]. EY. (70). appropriate fur the seepage conditions are evaluated in Q4SP and assembled in BC. (The FORTRAN variable corresponding to [.E] is DFLXQ.) Finally. the resulting banded system. EY. (67). is solved in routine SOLVE. Matrix [C] is decompesed into lower and upper triangular forms. and solutions of the two resulting matrix equations are obtained by forward and backward substitution.

Supporting calculations are carried out in subroutines SURF. FLUX, Q4D. SFLOW. Q4S, and Q4R. Subroutine SURF identifies boundary sides, which are then specified in terms of the elements to which they belong and the nodes that subtend them. Side kngths and direction cosines of outwardly directed normal vectors are calculated. Subroutines FLUX and QAD are used to determine nux vectors at all nodes from the predetermined concentration distribution. With SFI.OW. Q4S. and Q4R. Nows are determined in two different ways. First. the surface integral of Eq. (43) is evaluated for each boundary ekment in routine Q4S using two-point Gauss quadrature. Resulting element now rates are assembled over all boundary elements. and trapezoidal tinte integration is used in SFLOW to obtain the quantity of material passing through the boundary since the last time step. These flows are classified according to the boundary condition on the surface from which they originate. Sccond, space integrals over concentrations are evaluated in subroutine Q4R for each element. By performing appropriate sums in SFL.OW. quantities of material in liquid and solid phases and Insses through radioactive decay are determined. Thus, boundary nows may be compared with internal changes in material content to see that a balance has been achieved. 


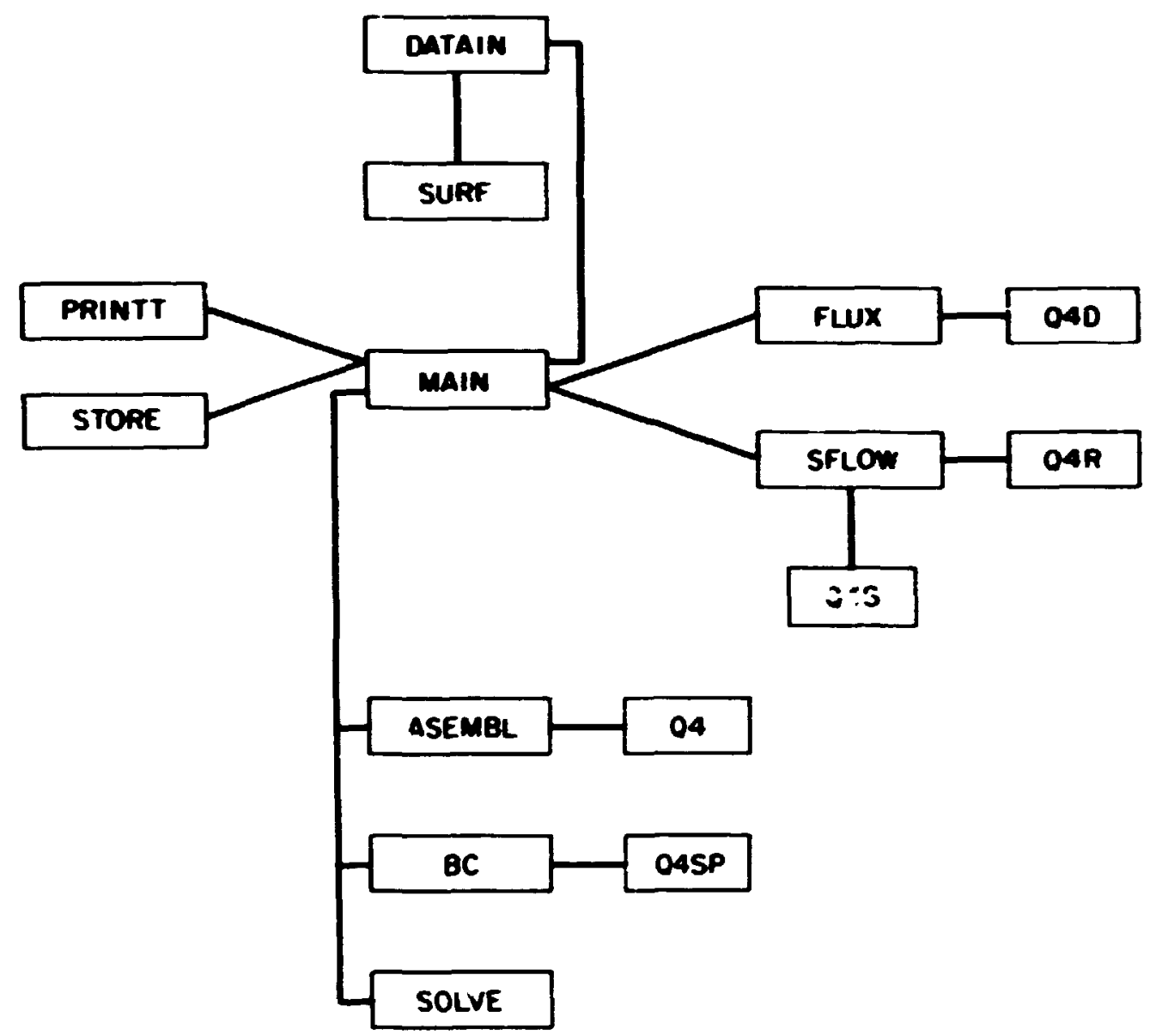

Fig. 4. Flow Chart of Discolved-Constituent Tmasport Program. 
ORNL-DWG 75-5574

\section{TIME LOOP}

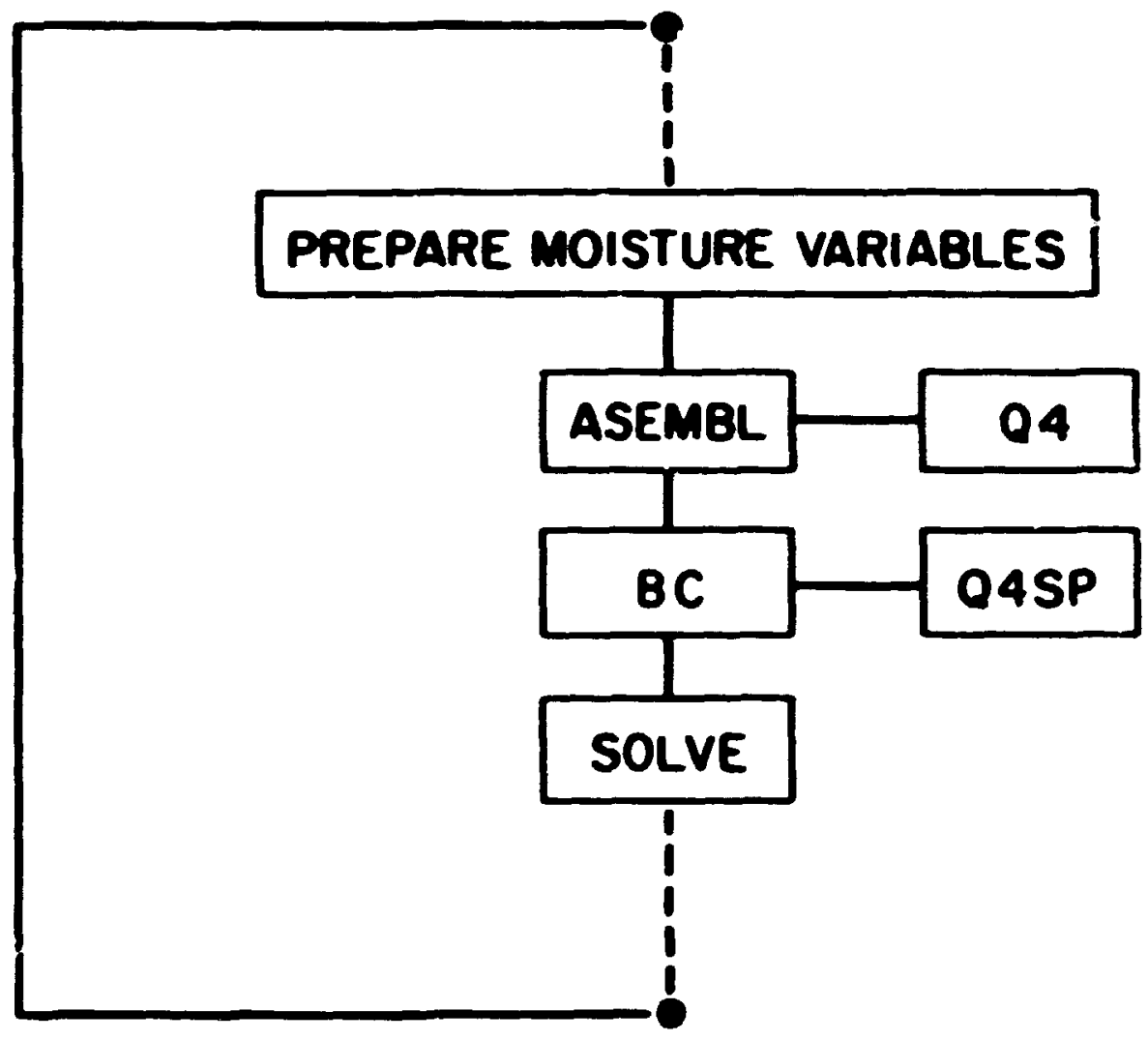

Fe. 5. Fhem Chan for the Tirm-lkention Loop. 


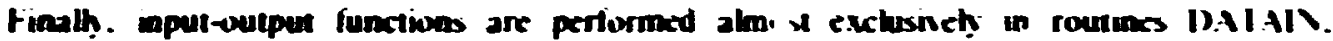
PRIXIT. and STORE. Varables pertaining to diseretivation of the exometry of ihe ssem and the simubion time are read in DAIAIX. Orher parameters rebting wo sol and

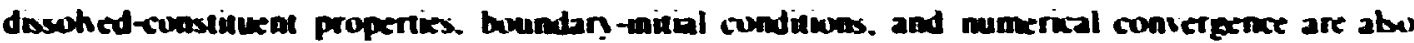

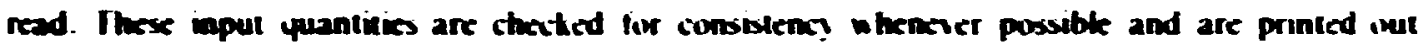
w give a complesc record of the simulation. A therough deser" : s enen in Appendix D. Onaput of calcubled lariabls accurs an PRIXIT and SIORE. Routine PRINTI prints how information. conceatrations. thuxes. and dorrubutions of maveluretranaport lariables as epecified by paraneter KPR. Routine STORE wrice the same informatimn. in addition to modatpoirt element descriptors. on an auxilan slordex dex such as a magnetic ape. Its operation is controlled bn variable KSTR. The soned information could then be used. lor example. as input is a photing program. 


\section{RESULTS}

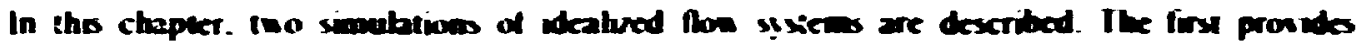

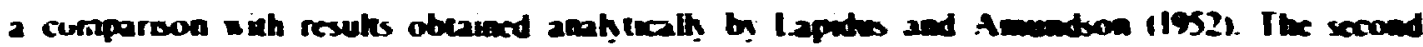

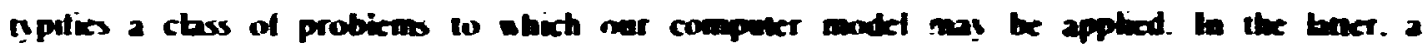

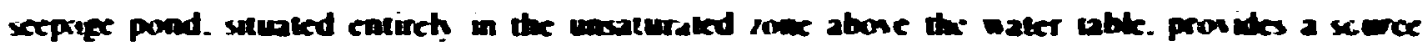

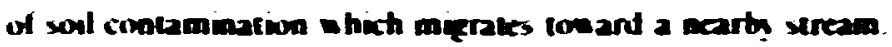

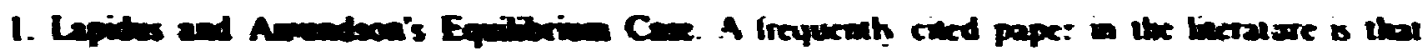

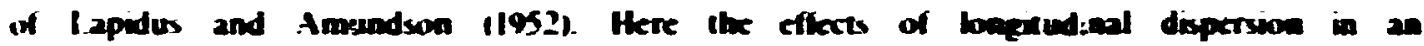

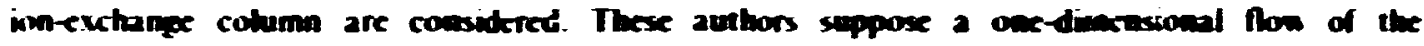

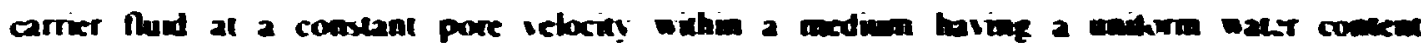
throughout. Since there is no radioactne decay. Ey. $(\mid 6)$ mal be oritien

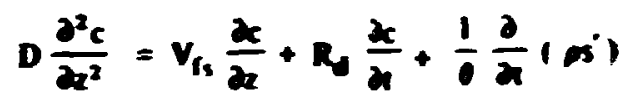

where the compressibilits $\mathrm{kem}$ is neglected and the pore velocity $1 . .=1 \% \theta$ is used rathet than

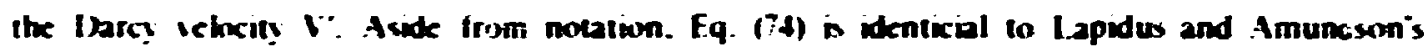
Fy. (1). For the case of prints ise cquilibrium and hearity. we use the rebion

$$
s^{\prime}=\frac{0}{n} k_{d i}
$$

I.apidus and Amundson add an arhitrary constant to the righthand side of Eq. (21) for increased exenerality. This is of no conseyuence. however. in the determination of $c$ since $s^{\circ}$ enters inin Fy. (?4) onty through a time derivative term. (Bulk density $p$ is. of course. ir dependent of time.) Combining E.ys. 121 ) and $(74)$ yieks

$$
D \frac{\partial^{2} c}{\partial z^{2}}=V_{1,} \frac{\partial c}{\partial z}+R \frac{\partial}{\partial x}
$$

in which $R$. is the retardation defined in Eq. (2.3).

With constait initial-houndary conditions

$$
c(2,1=0)=0
$$




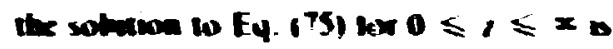

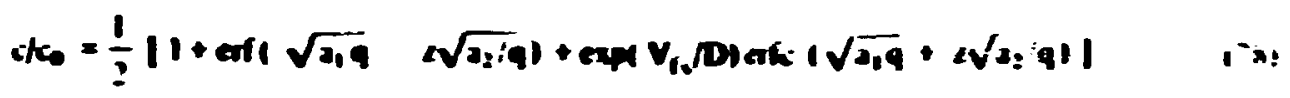

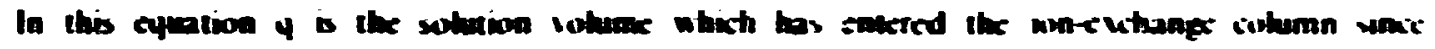
live $t=0$

$$
9=V_{1,} 10
$$

and the a's are constants defined $b$

$$
a_{1}=\left(V_{r_{8}}(D) / 4\left(R_{4}\right)\right.
$$

(XI)

and

$$
a_{2}=\left(R_{d} \theta\right)\left(V_{1 /} / D\right) / 4
$$

From Eqs. (78) throngh (8i) Lapidus and Amundson determine the reduced concentration c $c_{\text {. }}$ as a function of the solution volume 4 at depth,$=S O$ for seven different combinations of values of the parameters $R, \theta$ and $V, D$. Results from two of these analytical calculations. which they label as Curves $A$ and $E$. are reproduced in Fig. 6 along with results obtained from nur computer simulation. In both cases the reduced concentration remains at or near rero until hreakthrough is achieved. during which time it rises to a value approaching unity. Ohviously. the agreement of analytical and numerical rssults is fuite good. 


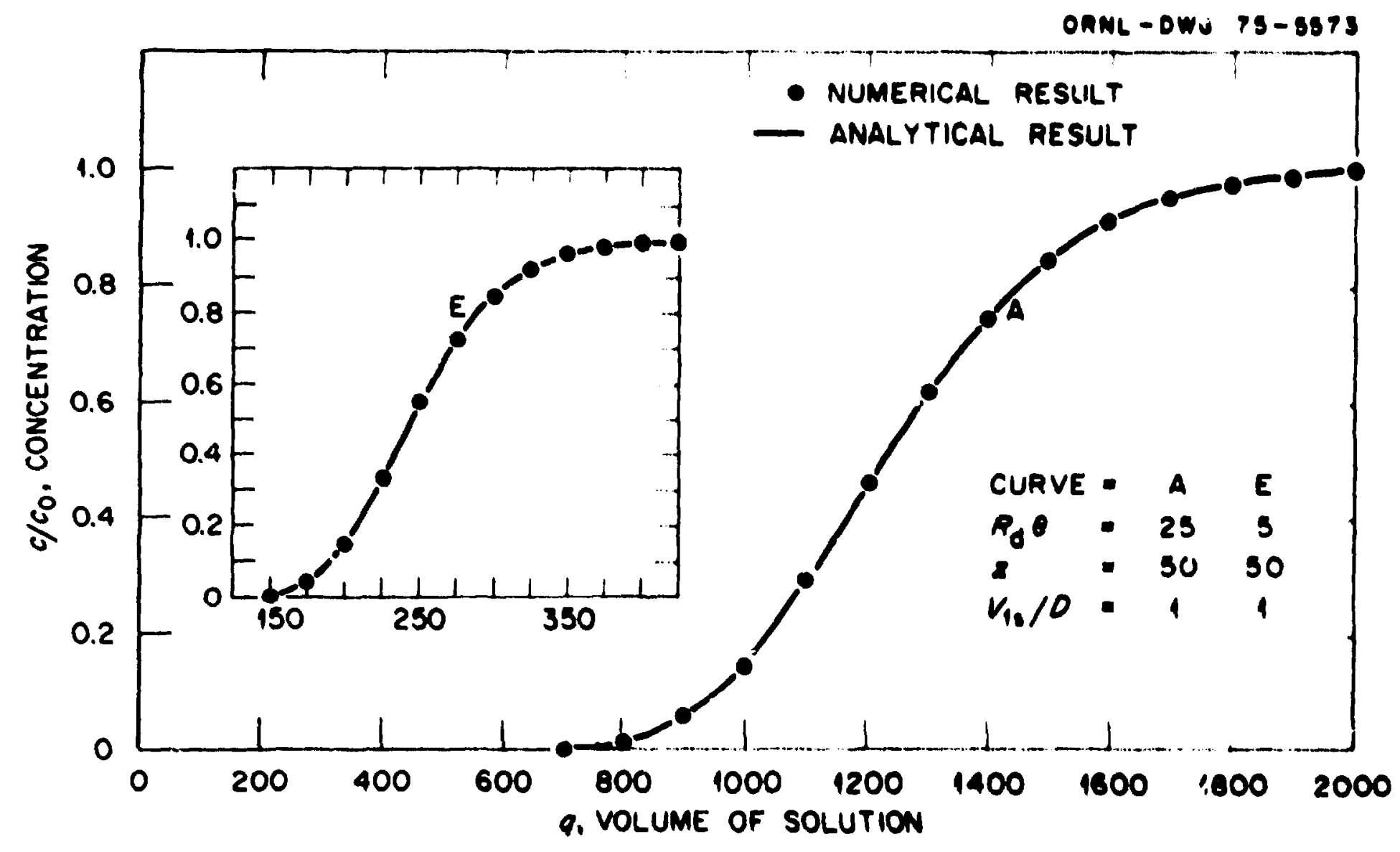

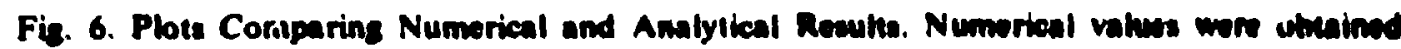
usine the computer code described in this repon. whereas anabytical valuen were obrained from the formula given by Lapidus and Amundion. 


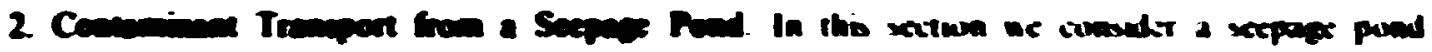

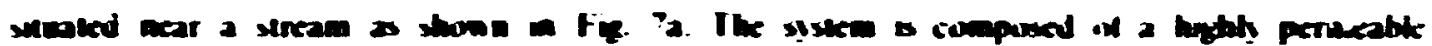

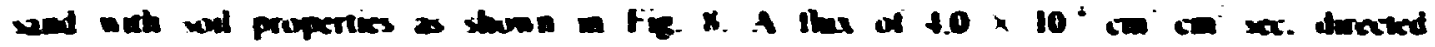

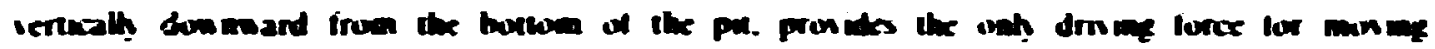

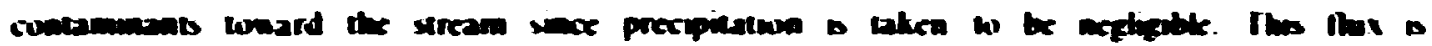

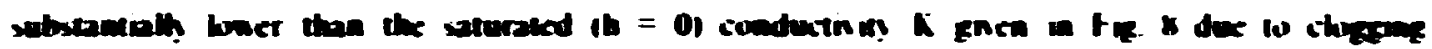

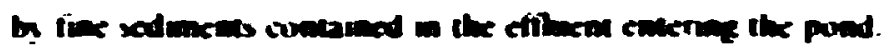

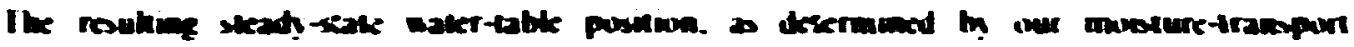

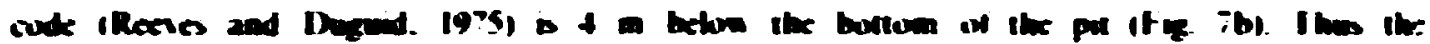

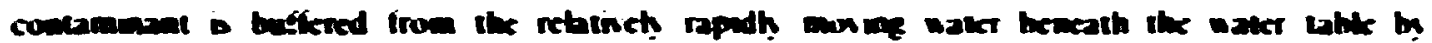

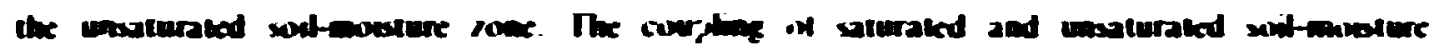

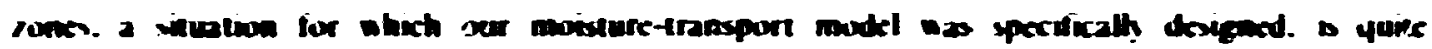

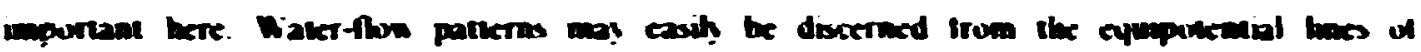

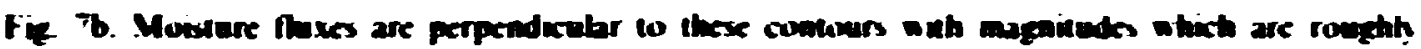

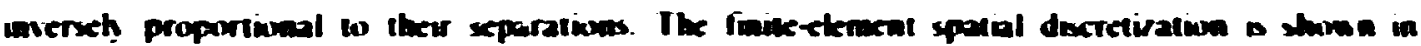
Fic. 7 .

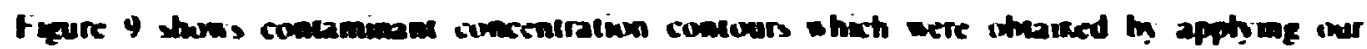

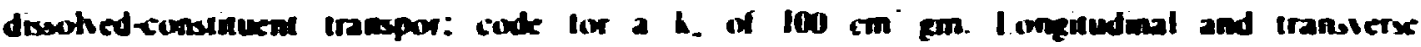

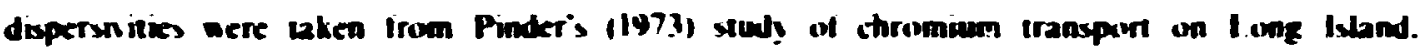
Wen York:

$$
a_{2}=2130
$$

and

$$
4^{4}=4.27 \mathrm{~m}
$$

The buffering effect of the unsaturated mil-moisture /one is apparent from the shapes of these contours.

As has been meentioned. two computer models have heen used to analyze the seepage-pond problem. The moisture-transport code (Reeses and Duguid. 1975) was used to obtain the Darcy velocities. Which were output on magnetic tape. These quantities were then input into the contaminant-transport computer model dexcrited in this report. Appendix A contains input to and output from the steady-state moisture-transport cakculation. Whereas Appendix B contains such information for the transient-state contaminant-transport calcislation. 

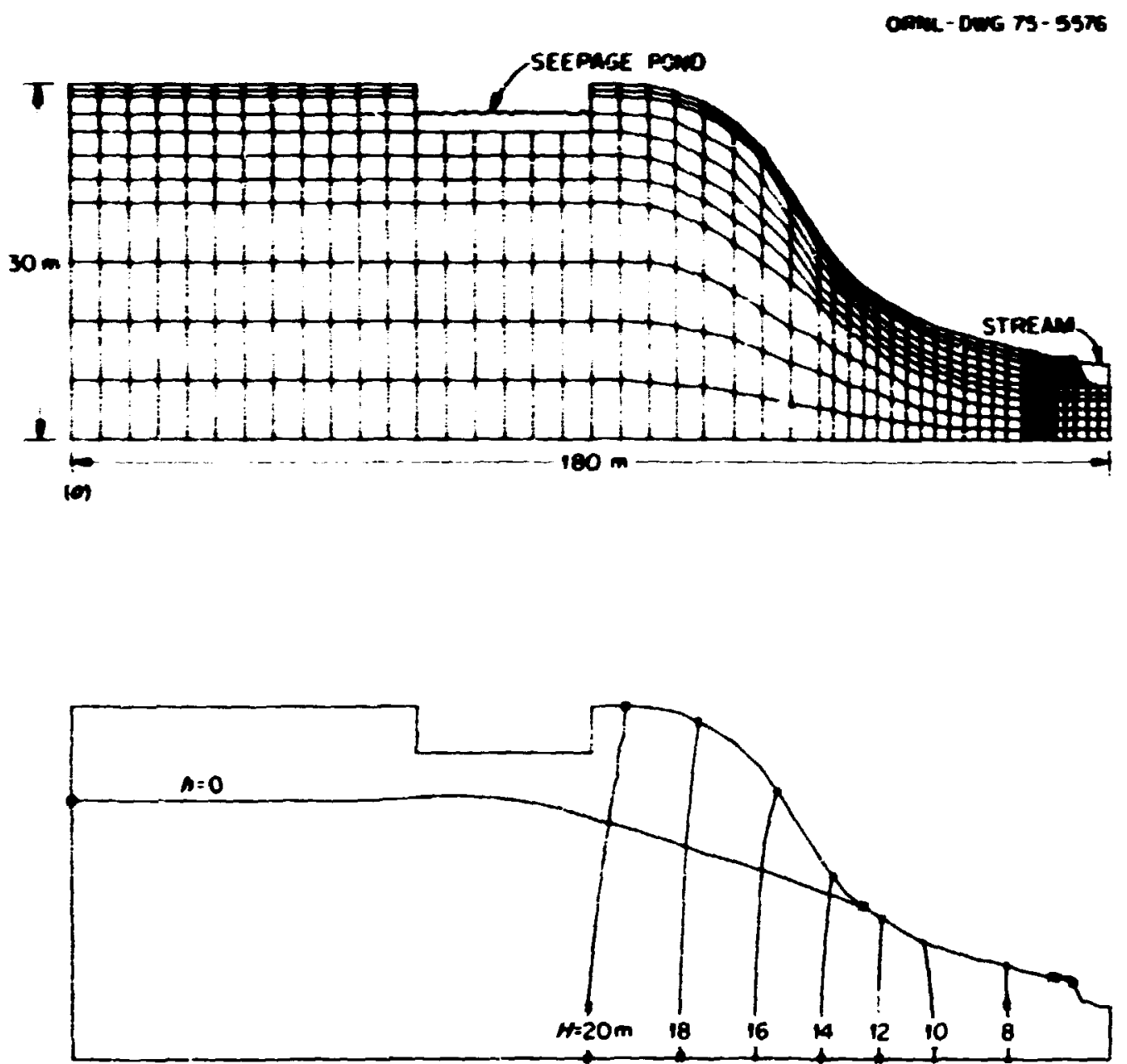

(b)

Fig. 7. (a) Spatial Discretization. (b) W'aser Table $(n=0)$ and Total Head (H) Configurations for Moisture Transpon Irom a Seepage Pond to a Stream. 


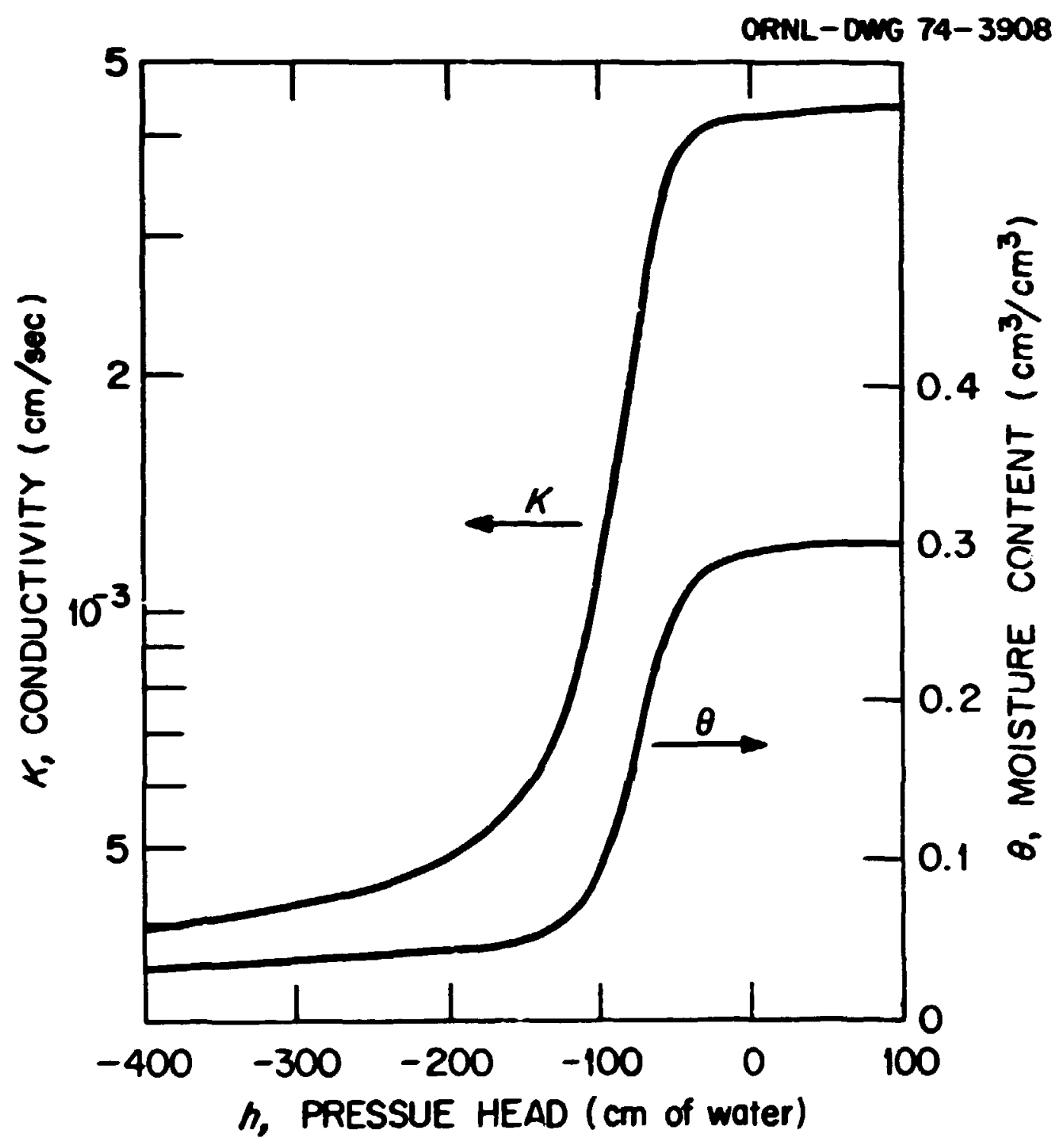

Fig. 8. Hydraulic Conductivity and Soil-Moisture Chalacteristics of a Hypothetical Sandy Soil. 

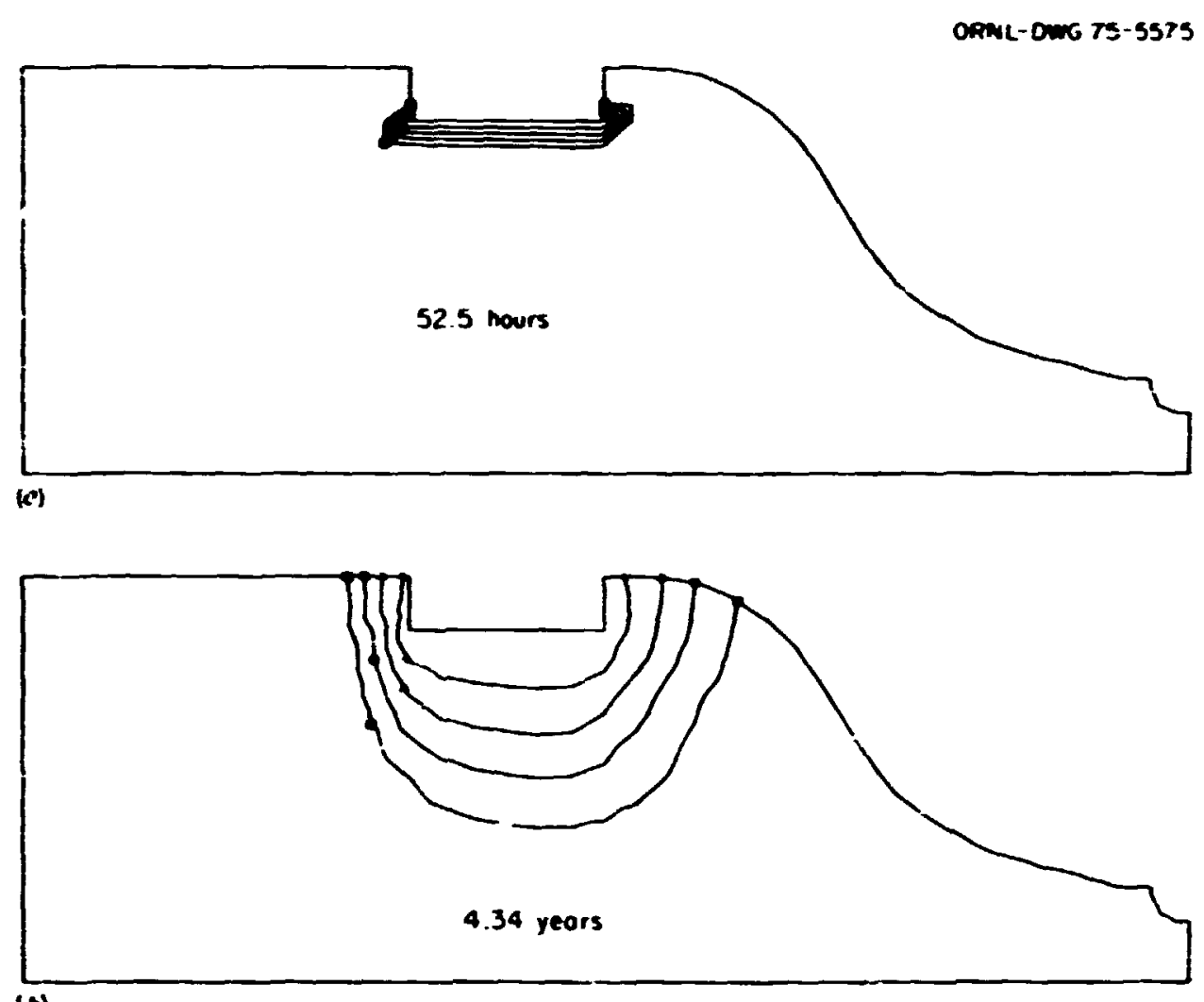

(b)

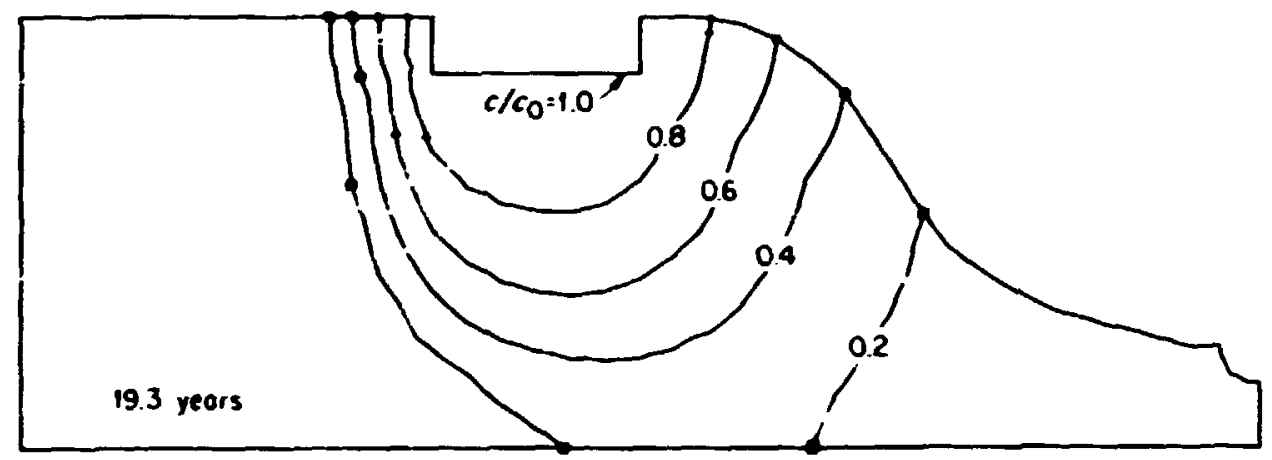

(c)

Fig. 9. Dispersion in Highty Permeable Sand of a Dissolved Constituent with a Distribution Coefficient of $100 \mathrm{~cm}^{3} / \mathrm{gn}$. 


\section{NOTATION}

Coefficient of the time derivative in the transpon equation

Integral of $A^{-}$over the volume of the $r$-th element

Coefficients of the time-derivative terms for the $r$-th ekment

Longitudinal dispersivity

Mokcular diffusion coefficient

Transverse dispersivity

Coefficient of the concentration in the transport equation

Integral of $\mathrm{B}^{-}$over the volume of the r-th element

Coefficients of the concentration terms for the r-th ekment

Dirichkt boundary condition

Assembled operator matrix for all ekments

Combination of $[, A]$ and $[, B]$ including the time-integration algorithm

Conceniration of the dissolved constituent

Time derivative of the concentration

Assembled values of the unknown coefficients

Unknown coefficients for the r-th element

Time derivative of $\mathbf{c}$

Hydrodynamic dispersion tensor

Ekment matrix containing surface integrals appropriate for secpage boundary conditions

Dilatation of the medium 


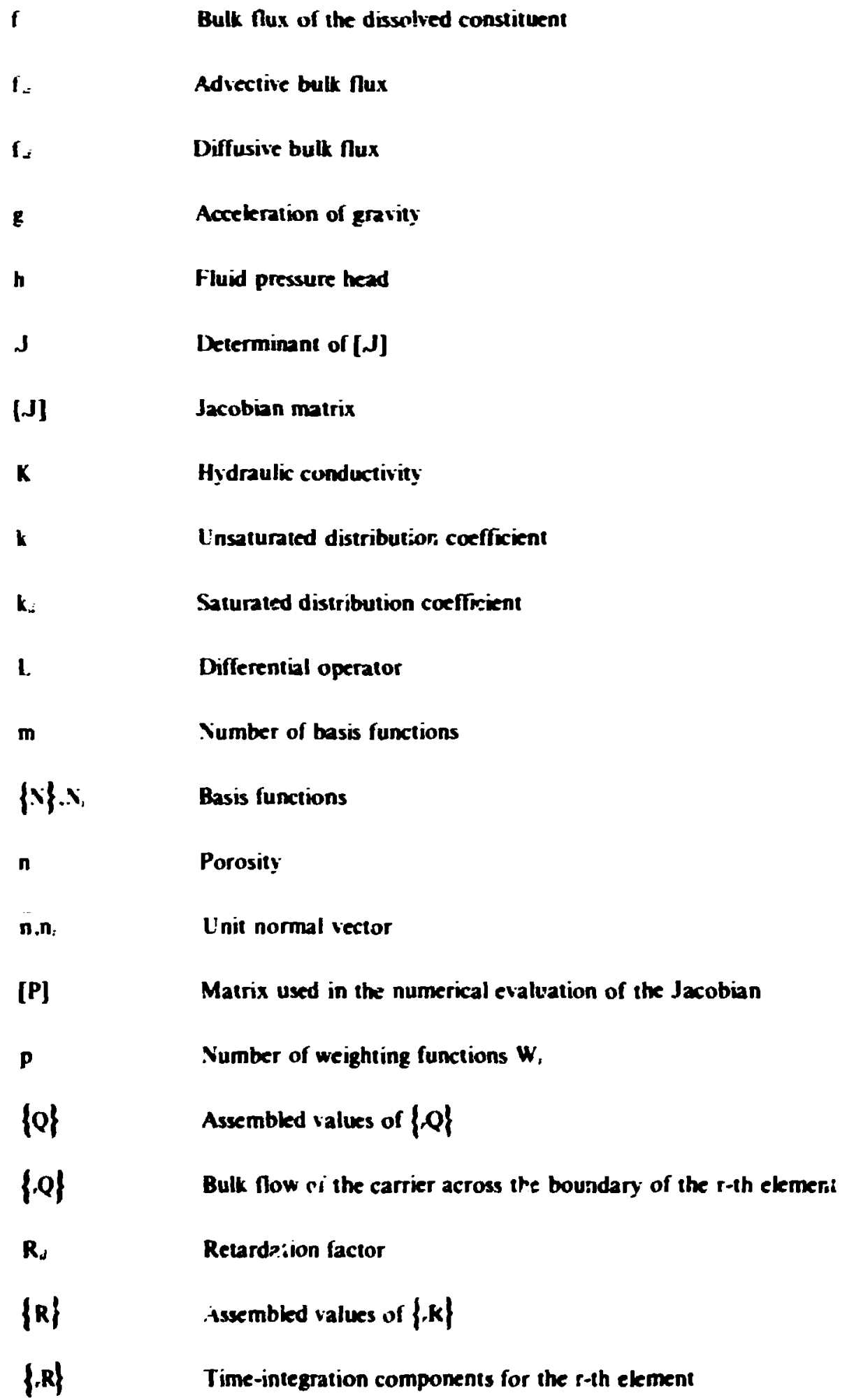


$\mathbf{s}$

5.7

S.T.

$s^{\prime}$

$\mathbf{t}$

$v$

, V

$\overline{\mathbf{v}}_{\text {, }}$

$\overline{\mathbf{v}}, \mathbf{v}$

v'

$\overline{\mathbf{v}}$

w,

$\overline{\mathbf{x}}, \boldsymbol{x}_{1}$

$\boldsymbol{x}$

-X

$\mathbf{X}_{\boldsymbol{r}}$

$\{, x\}$

$\{r\}$

7

7

7.4
Refe:s to the r-th finite element

Surface area of the r-th ekment

Local coordinates

Local coordinates of the nodes $j=1,2,3$, and 4

Solid-phase concentration of adsorbed constituent

Time

Volume of the system

Volume of the r-th element

Pore velocity of the carrier rebative to the solid

Darey Velocity of the carrier

Magnitude of Darcy velocity

Velocity of the solid

Weighting function

Position vector

Lateral coordinate

Global coordinate of a point within the r-th ekement

$x_{1} \cdot x_{1}$

Global coordinates of the nodes of the r-th element

Assembled load vector containing boundary fluxes and time-integration components

Vertical cor rdinate

Global coordinate of a point within the r-th etement

7. ? 


\begin{tabular}{|c|c|}
\hline$\{, 2\}$ & Global coordinates of the nodes of the r-th element \\
\hline a & Coefficient of compressibility of the medium \\
\hline $\mathbf{a}^{\prime}$ & Modified coefficient of compressibility of the medium \\
\hline st & Time increment \\
\hline $\mathbf{\epsilon}_{7}$ & Strairs :ensor for the medium \\
\hline $\boldsymbol{\theta}$ & Moisture content \\
\hline $\boldsymbol{\lambda}$ & Radioactive decay consian: \\
\hline $\boldsymbol{\rho}$ & Bulk density of the medium \\
\hline $\boldsymbol{p}_{\mathbf{f}}$ & Density of the carrier fuid \\
\hline$r^{\prime}$ & Tortuosity \\
\hline$\Phi$ & Unknown solution to the equation $L(\Phi)=0$ \\
\hline$\phi^{*}$ & Trial solution \\
\hline.$\Phi$ & Trial solution for the $\mathrm{r}$-th element \\
\hline $\boldsymbol{\phi}$ & Expansion coefficients relative to the basis functions: \\
\hline & Time-integration parameter \\
\hline
\end{tabular}




\section{REFERENCES}

Bear. J., Dinamixs of Ftuids in Poruus Medio. American Ekevier Publishing Co.. Inc.. New Yort. 1972.

Bredehoeft. J.. Comment on Numerical Solution to the Convective Diffusion Eyuation by C. A. Oster. J. C. Sonnichsen. and R. T. Jaske.- Haker Reswour. Res., 7. 755-757. 1971.

Bredehoeft. J.. and Pinder. G., -Mass Transpon in Flowing Groundwaler." Hater Resume. Res.. 9. 194-210. $19,3$.

Desai. C.. and Abel. J.. Mrrocherion to the Finice Ekment Mechou: A Numerixal Methed for Enincering Amalysis. Ian Mostrand Reinhold. New York. 1972.

Finlayson. B.. The Method of Meighed Residuals and liariorional Prinriples. Academic Press. New York. 1972.

Hutton. S.. and Anderson. D.. -Finite Element Method: A Gakrkin Approach." J. Eng. Mrch. ASCE. 96. 1503-1519. 1971.

Lai. Sung-Ho, and Jurinak. J.. -Numerical Approximation of Cation Exchange in Miscible Displacement through Soil Columns.- Soil Sri. Sox. Amer. Pror.. 35. 894-899. 1971.

Lai, Sung Ho. and Jurinak. J., 'Cation Adsorption in One-Dimensional Flow through Soils: A Numerical Solution." Hacer Resour. Res.. 8. 99-107. 1972.

Lapidus. L.. and Amundson. N.. Mathematics of Adsorption in Beds. VI. The Effect of Longitudinal Diffusion in Ion Exchange and Chromatographic Columns." J. Phis. Chem.. 56. 984-988. 1952.

Makem. L.. Introduction to the Mechanics of a Continuous Medium. Prentice-Hall. Inc.. Englewood Cliffs. I. J.. 1969.

Misra. C.. Nielsen, D. R., and Biggar. J. W.. "Nitrogen Transformations in Soil during Leaching: 1. Theor.tical Considerations." Soil Sci. Sor. Amer. Proc.. 38, 289-293. 1974.

Ostet. C.. Sonnichsen. J.. and Jaske. R.. -Numerical Solution to the Convective Iiffusion Equation." Hater Resour. Res.. 6. 1746-1752. 1970.

Pinder. G.. “A Galerkin-Finite Ekment Simulation of Groundwater Contamination on I.ong Island. New York.- Water Resour. Res.. 9. 1657-1670. 1973. 


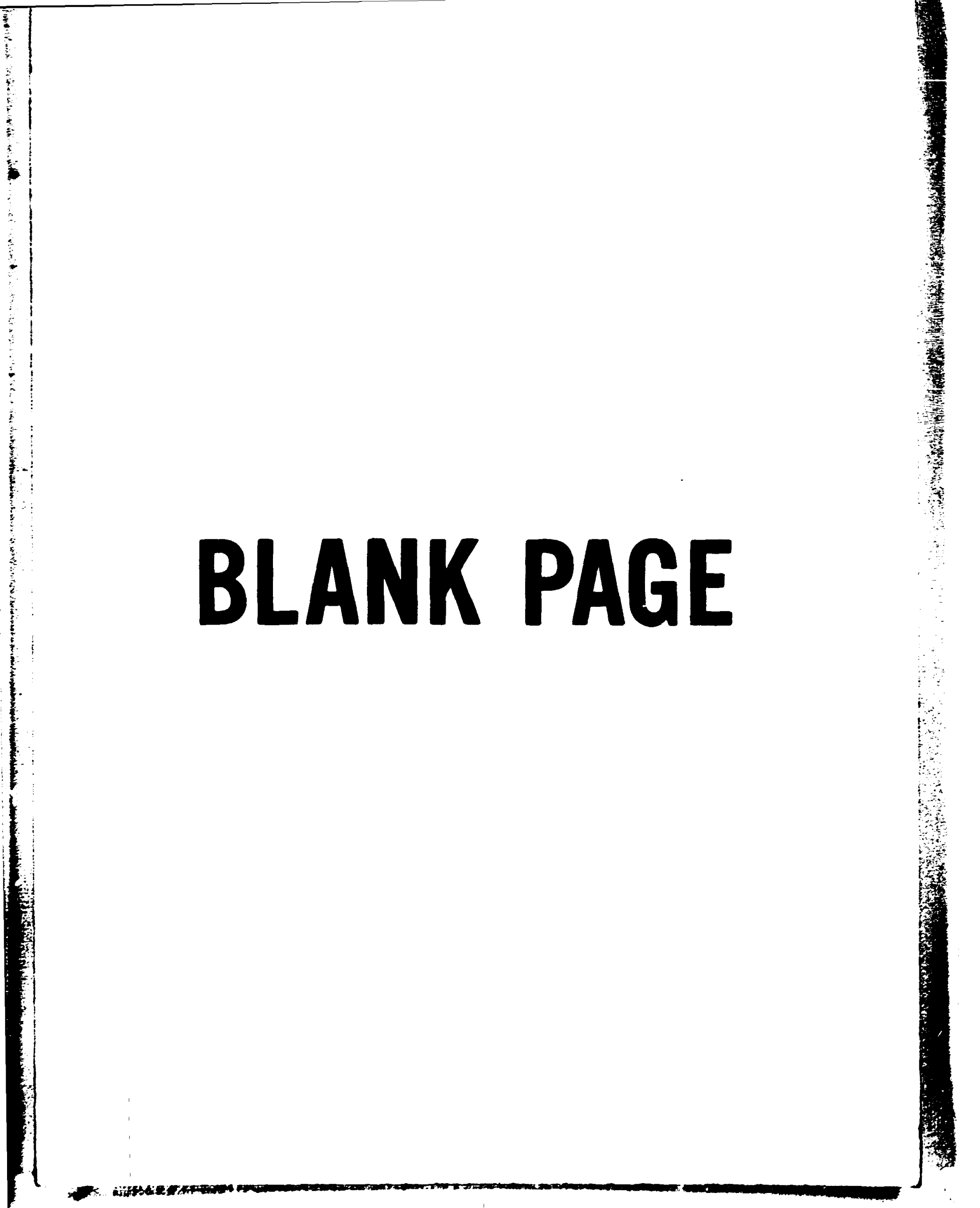


Reeves. M.. and Duguid. J.. Hater Movement through Saturated-Linsaturated Pirsus Mtedio: ti Finite Ekment Gakrtin Mockl. Oak Ridge Vational Labortory. Oak Ridge. Tenn.. ORNL 4927.1975.

Rubin. J.. and James, R. V.. -Dispersion-Affected Transport of Reacting Solutes in Saturated Porous Medin: Calerkin Methods Applied to Equilibrium-Controlled Excharge in Inidirectional Steady Water Flow; - Haie? Resour. Res.. 9. $1332-1356.1973$.

Van Genuchten, M. T.. Davidson. J. M., and Wieteng. P. J., An Evaluaion of Kinctic and Equilibrium Equations for Meda.- Soil Sri. Sor. Amer. Pror.. 38. 29-35. 1974. 


\section{APFENDD A}

\section{NPUT AND OUTPUT FOR SEEPAGE-PT MOSTURE TRANSRORT}

This appendix presents input to and output from the computer program documented in [Reeves and Duguid. 1975]. The moisture-transport variables obtained in this calculation were printed on manenetic lape by subroutiue STORE and then read as input for the dissolied-constituent-transport determination (Appendix B). Subroutine STORE of the above-mentivned program has been modified to be more compatible with the dissolved-constituent code. An updated listing is therefore included at the has of the section on input. 
INPUT 

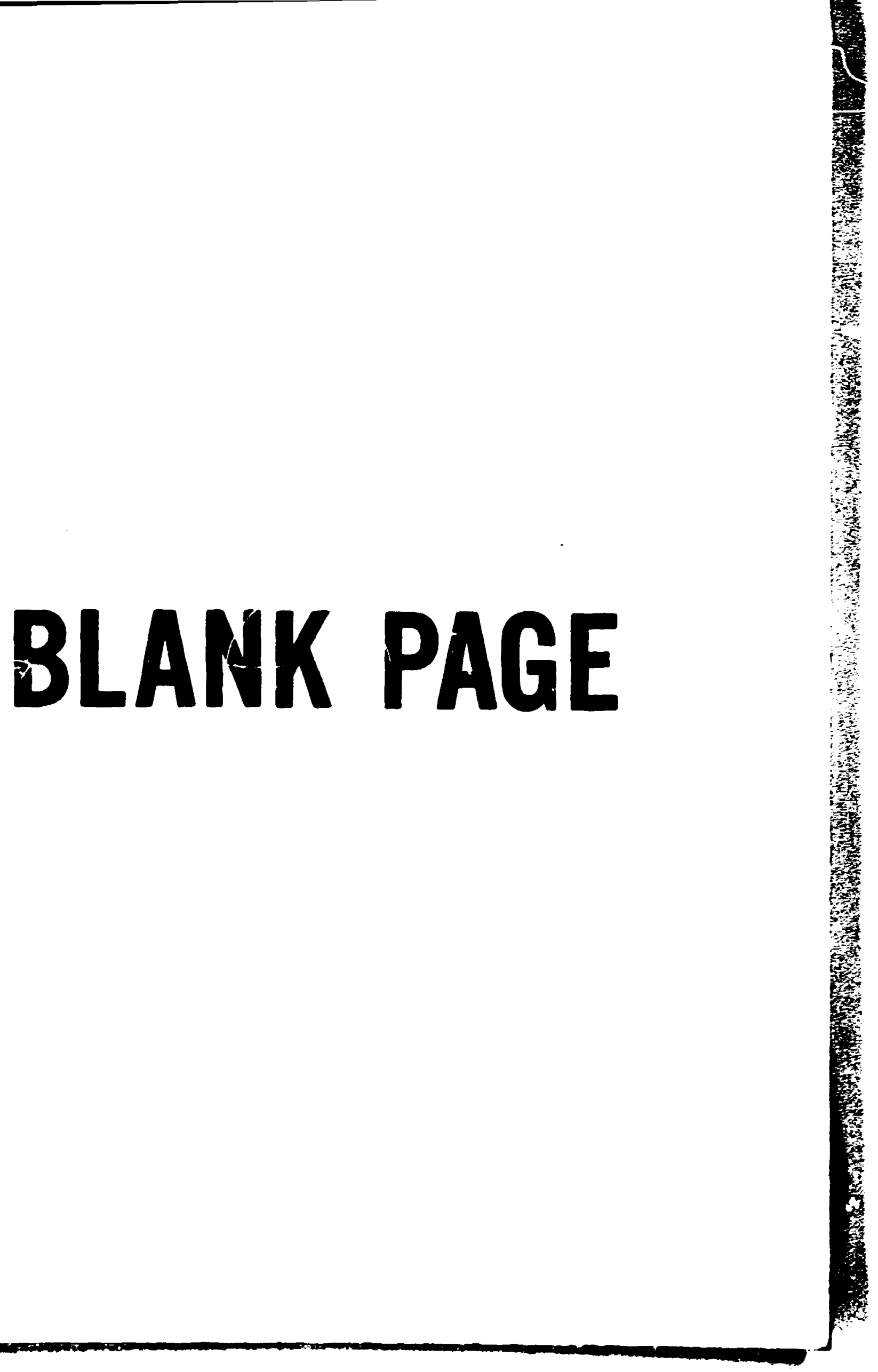


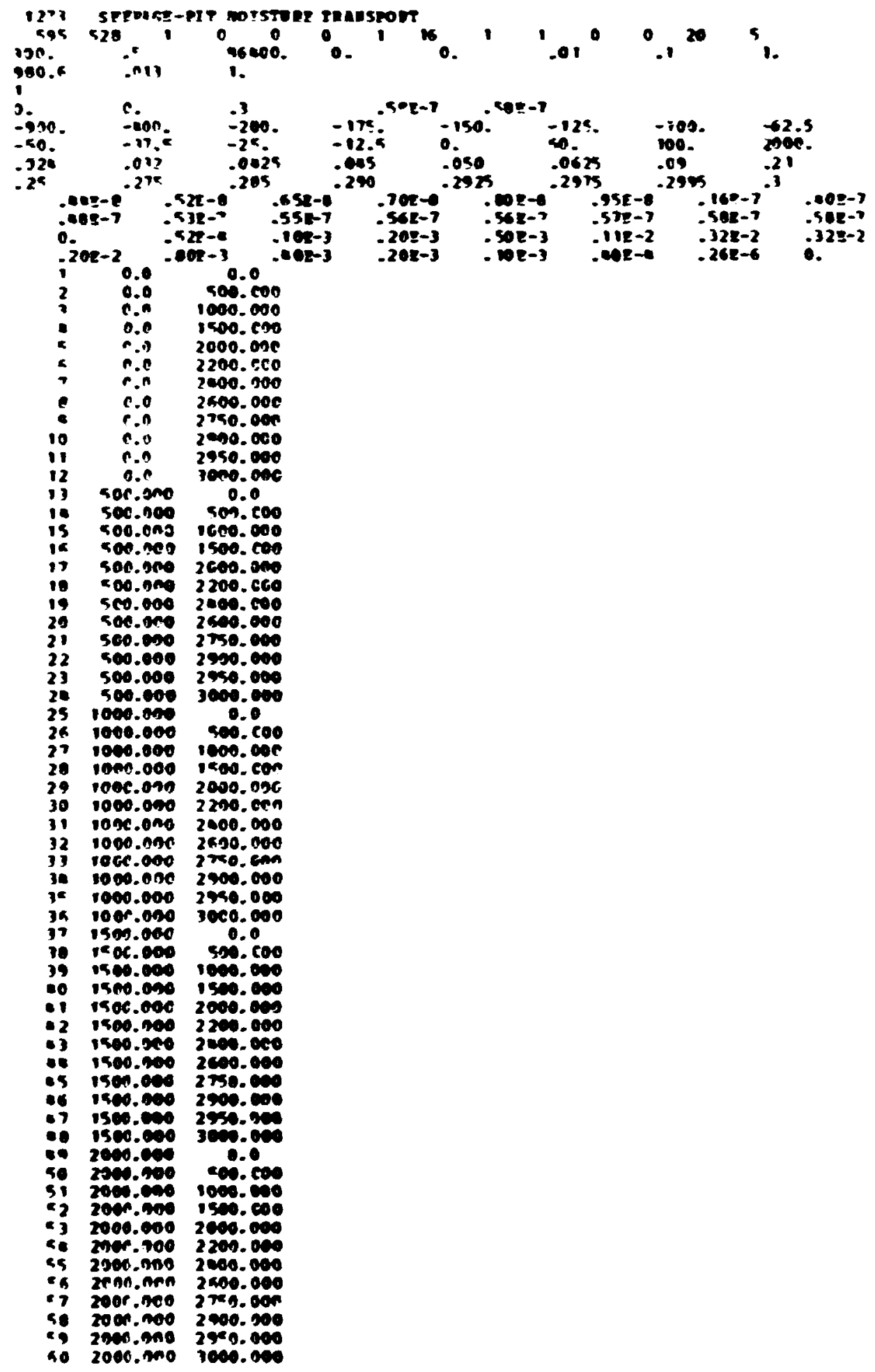




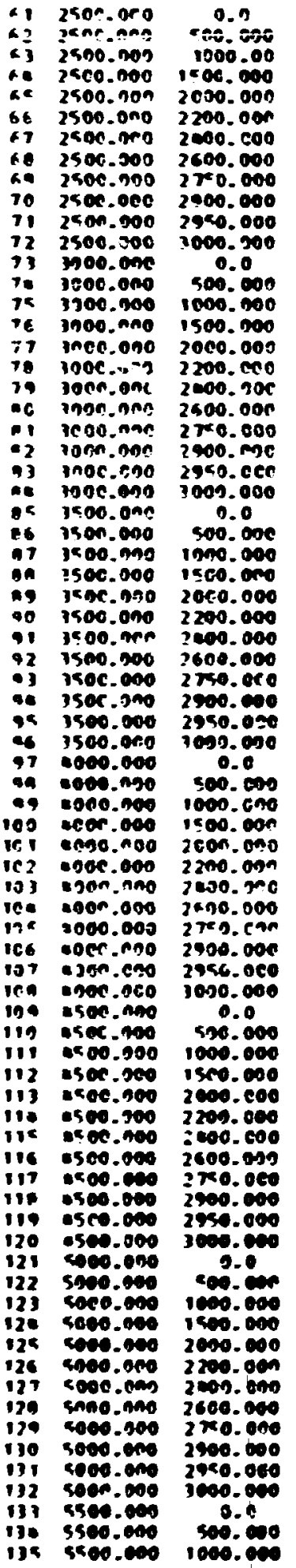




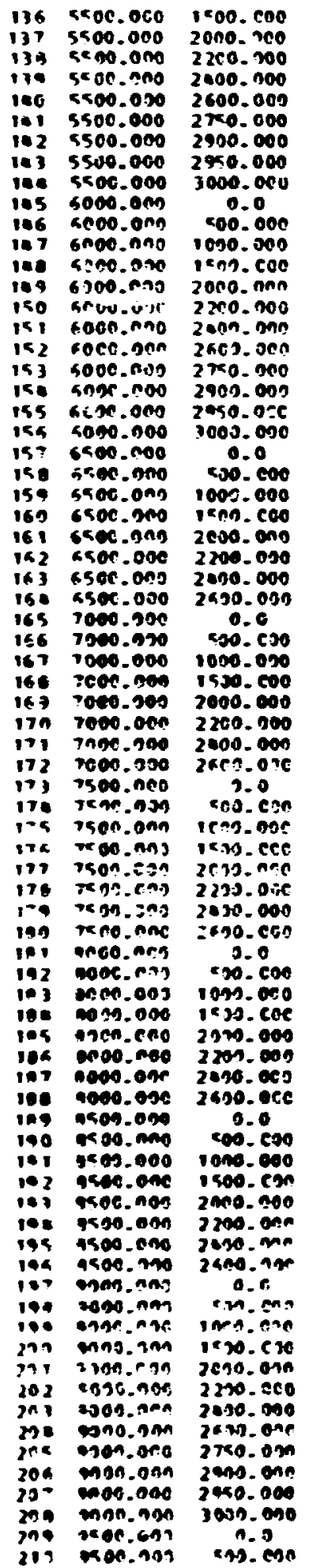




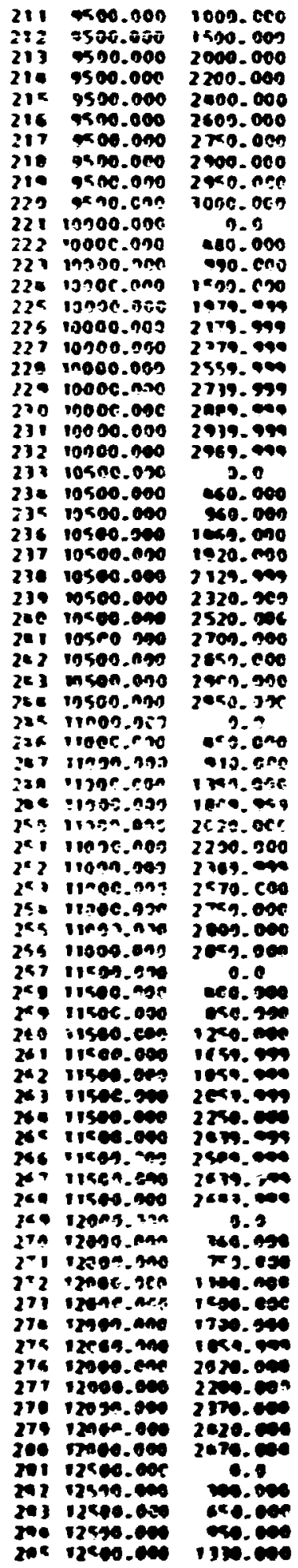




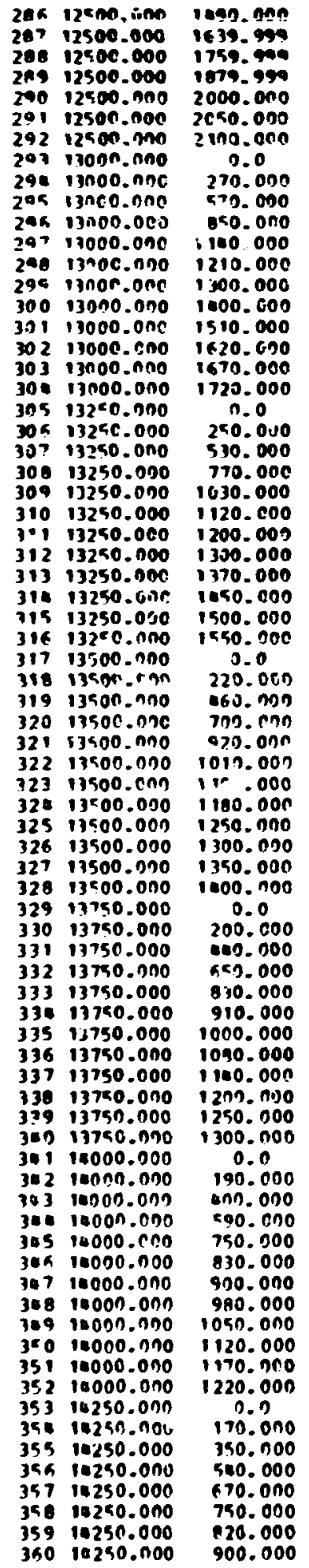




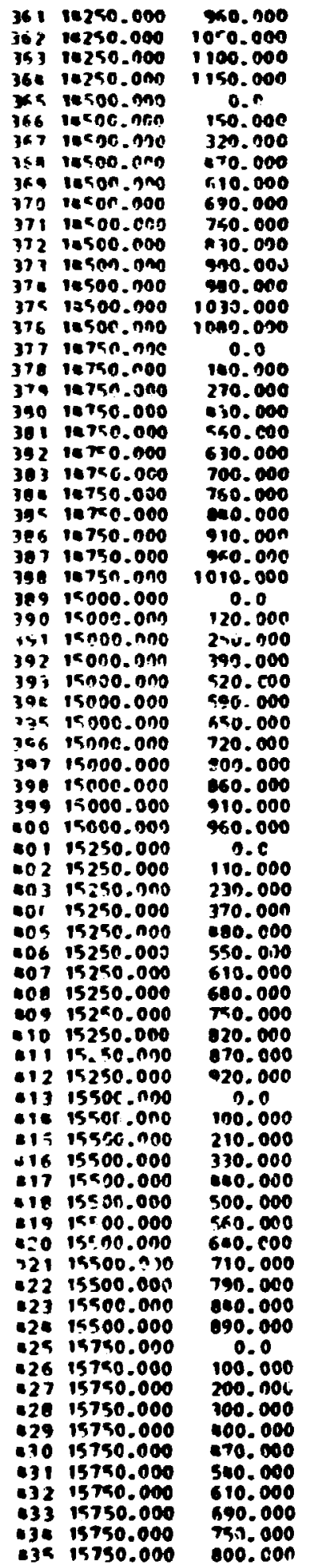




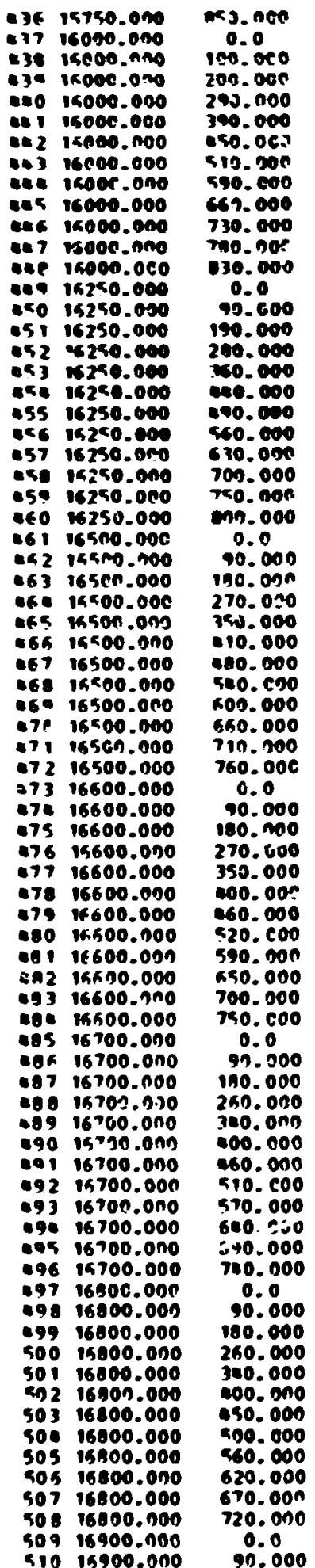




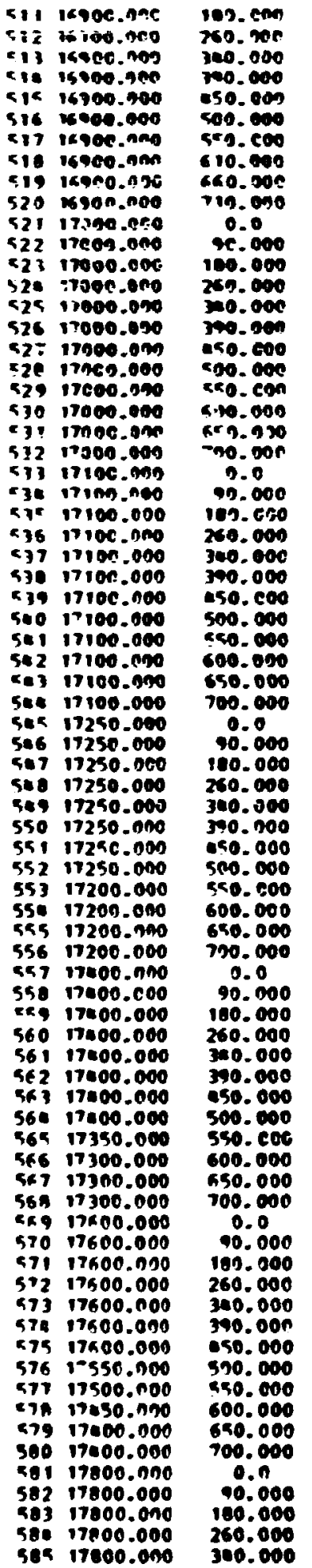




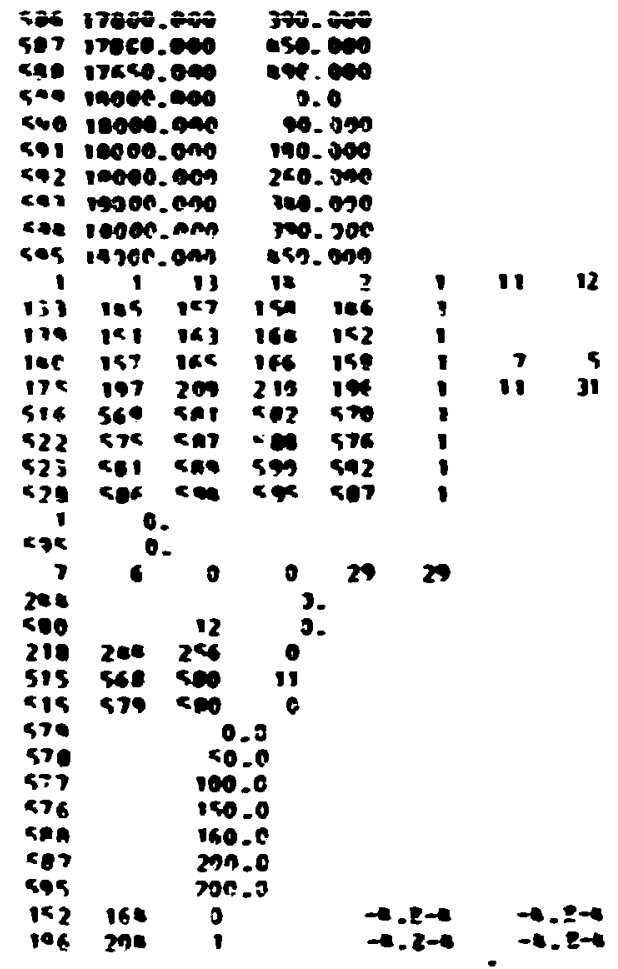


$c$
$c$
$c$
$c$
$c$
$c$
$c$

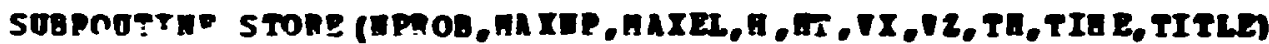

STOR

STOR

STOR

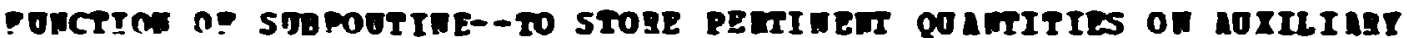

STO:

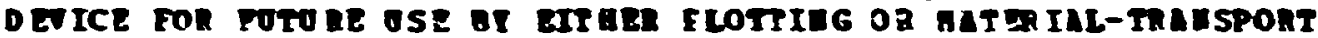

15

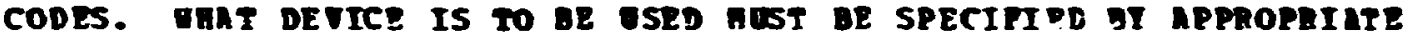

jo-control caros.

IAPLICIT RenLes $(n-\pi, 0-z)$

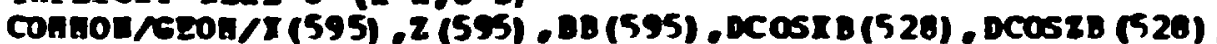

STOR 25

STOR 30

STOR 35

STOR 10

sron

45

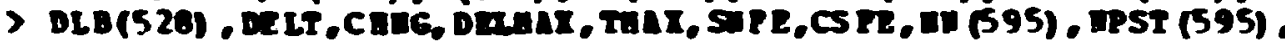

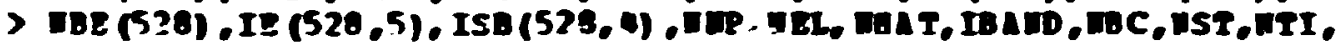

$>$ moEt, ESTH, PSTR?

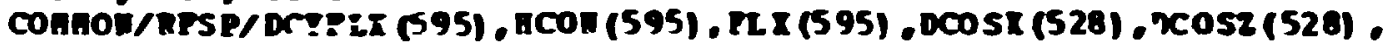

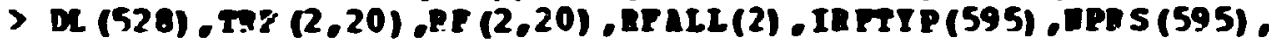

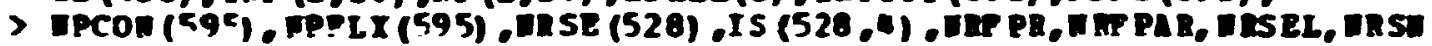

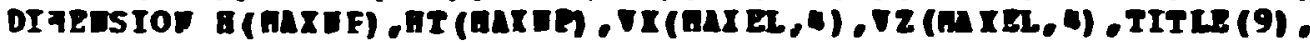

$>$ TH (nATEL, G)

DATL IPP \$OB/ $-1 /$

IP (ISTE:.GT.0) 60 To 10

IP (IPPYOE.RQ. $(-1)$ ) RETIDD I

IF (UPPPOB. Ec. DP

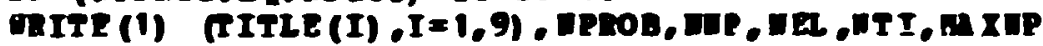

S:J8 90

sive 95

STOR 100

STOR 105

STOR 110

STOR 115

$>1,01$

EPRROS= IPROS

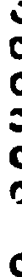

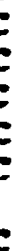

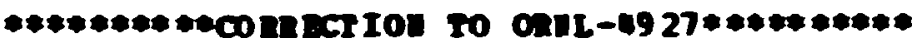

STOR 130

STOR 135

STOR 140

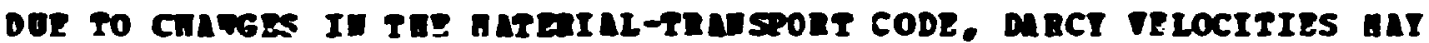

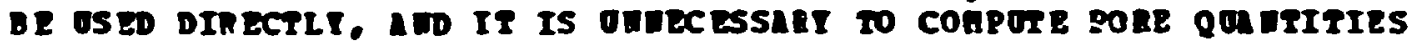

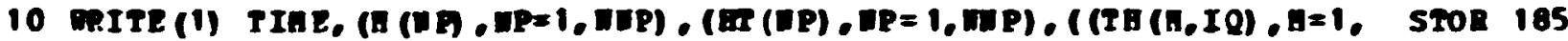

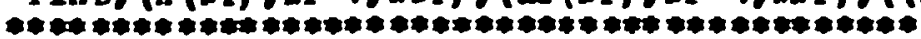

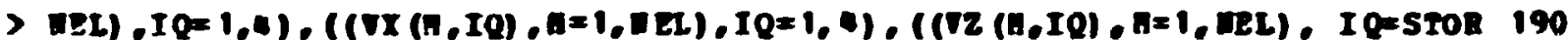

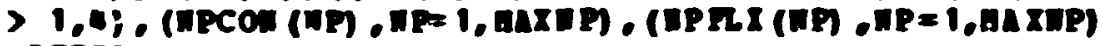

S20R 195

RETORN

STOR 200

END

STOR 205 
OUTPUT 


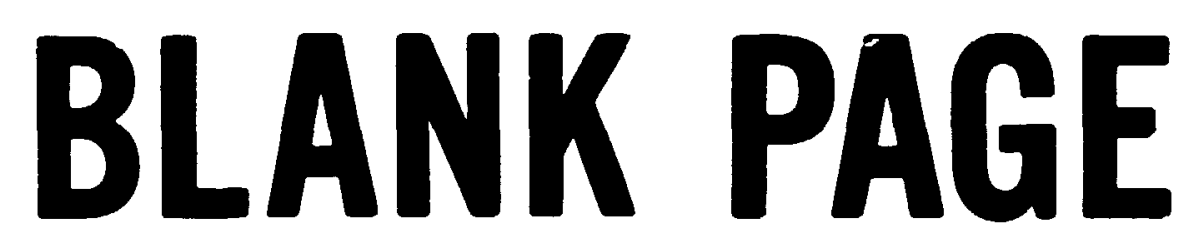




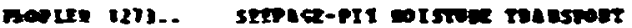

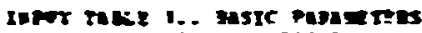

corer of mol motes.

Deses or zeters.

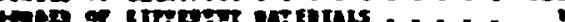

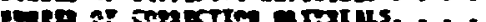

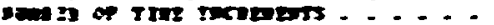

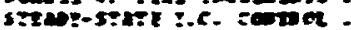

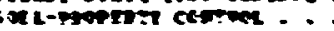

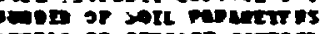

-

Gearre come

errer pasare: : : : : :

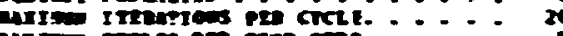

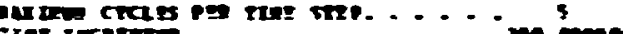

ira Ixse

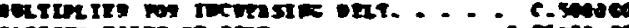

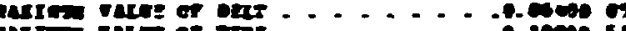

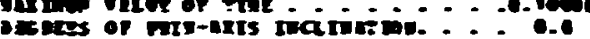

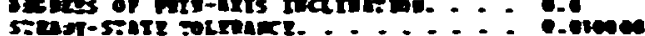

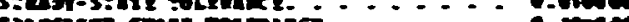

rassics:-smet rekedece

cossrt s carpo

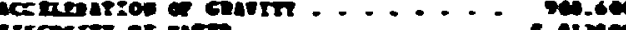

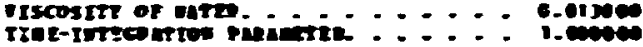

orrer corren

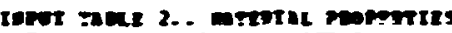

ㄷ.

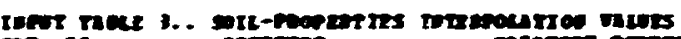

ter. 10.

- - cosen 3

-e.even

$-0.21+46$

- itris

e.326en-1

- expren

-issen-1

- -staper

- - -1350

- o. 1en

-.6rin-1

- i.criat

-

- - . sures a

- 2160

- - istos es

$-0.12505$

0.0

-zose es

. 2060

$0.2925 \%$

.5000 oz

c.2006

o.zrive w

asear-e

conctivithughotitr

c. mev-

9.52410

$0.6500-1$

e. 7ove-

- 6010-4

$0.730-6$

- acer-er

- Steverar

- secter

c. stem-17

- snower

- sever-1

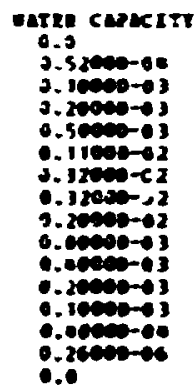




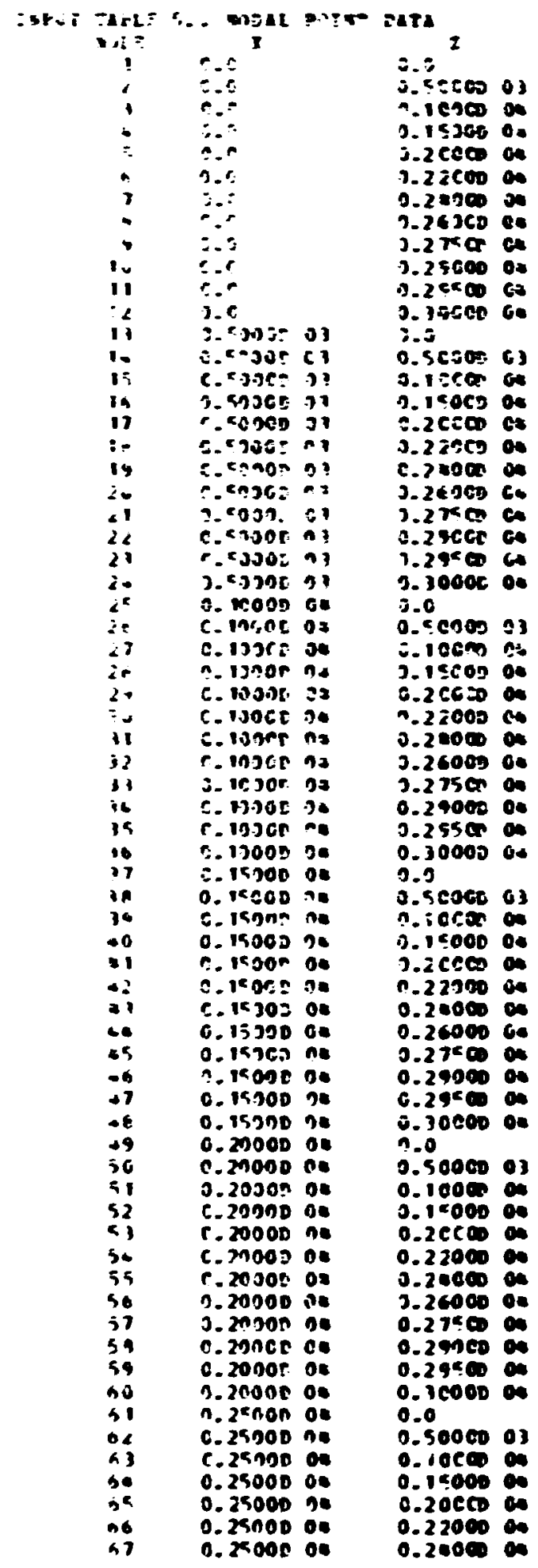




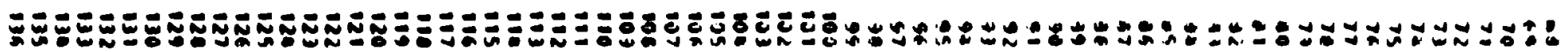

ט 申

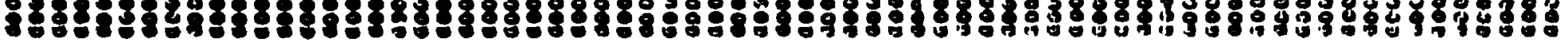

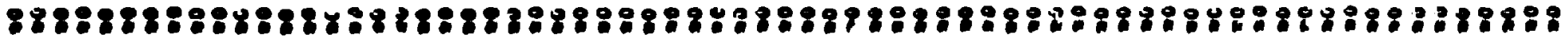

"

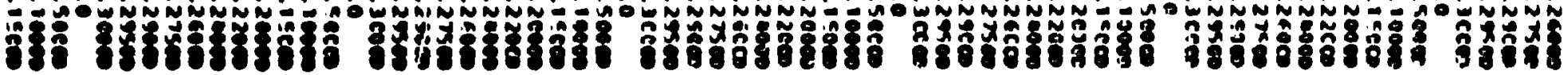

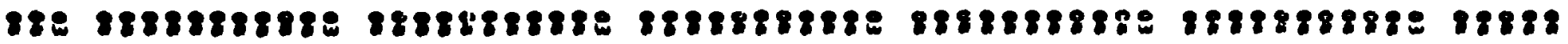




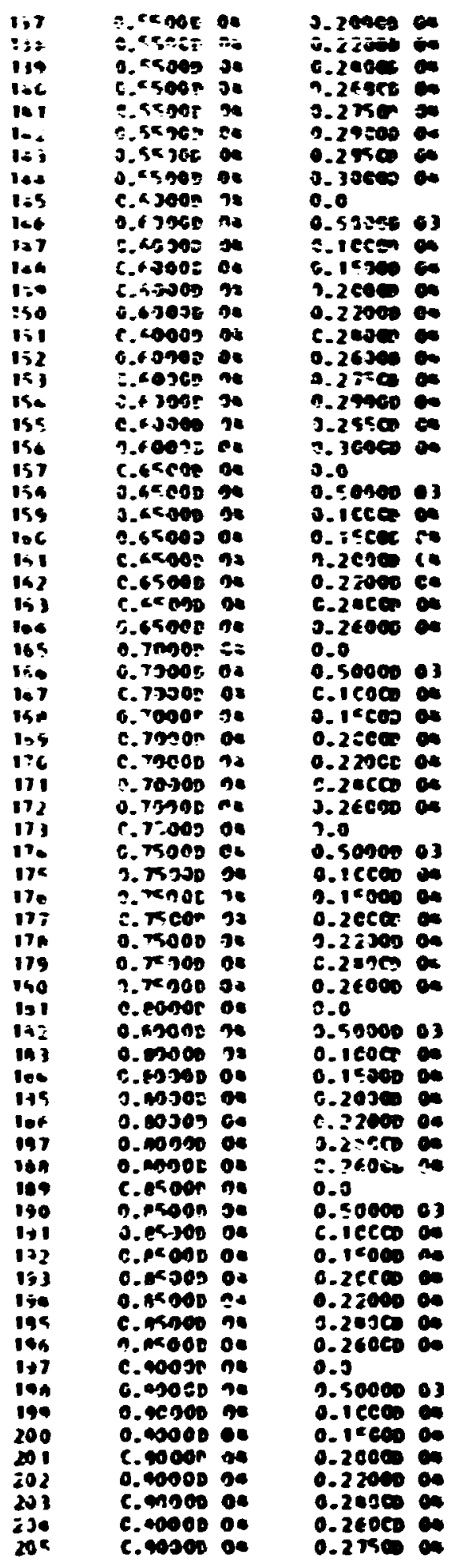




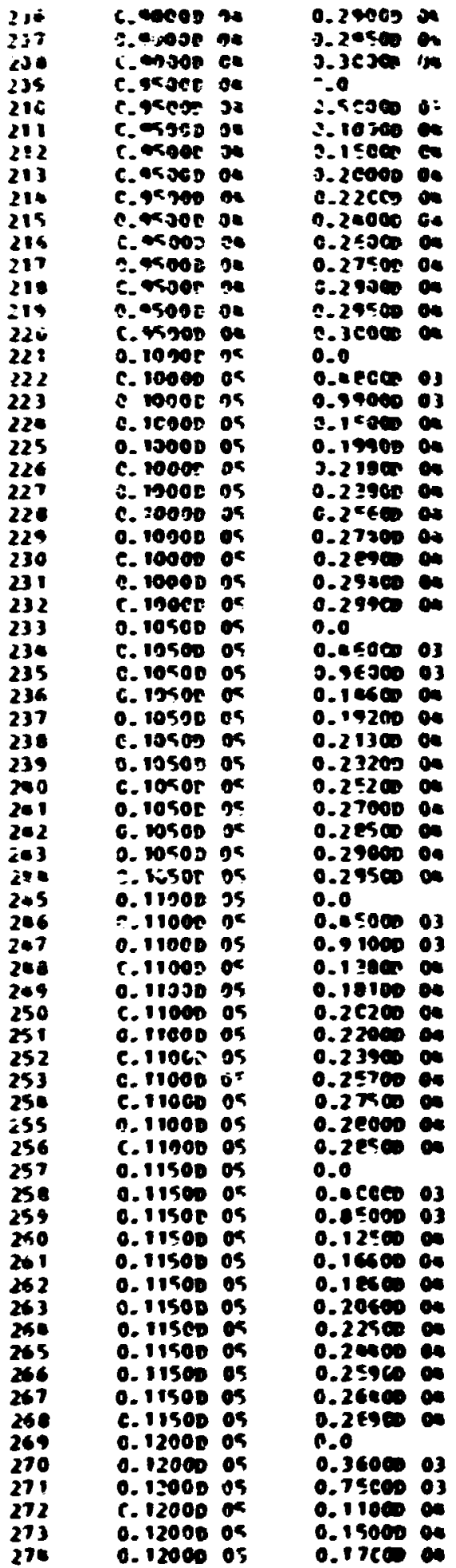




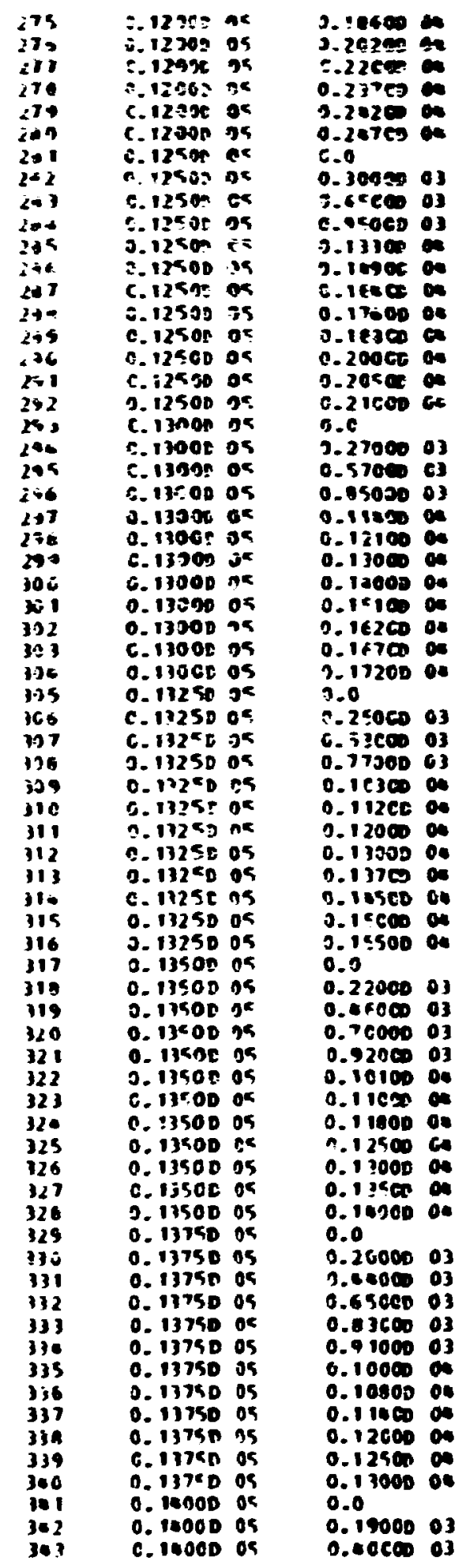




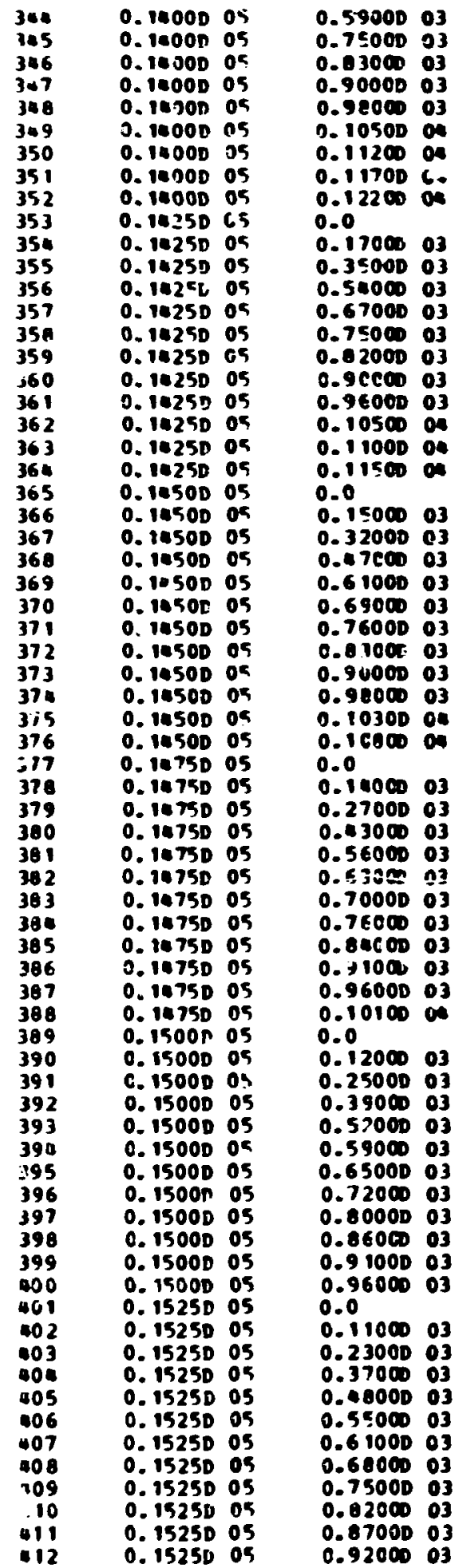




\begin{tabular}{|c|c|c|c|}
\hline 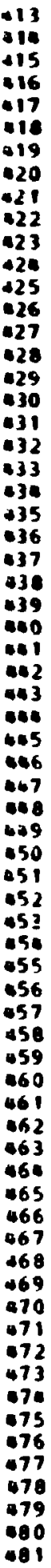 & 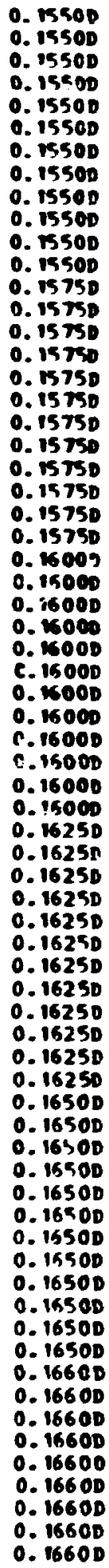 & 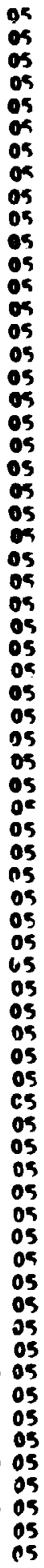 & $\begin{array}{l}0.49000 \\
0.56000 \\
0.63000 \\
0.70000 \\
0.75000 \\
0.80000 \\
0.0 \\
0.90060 \\
0.10000 \\
0.27600 \\
0.35000 \\
0.11000 \\
0.18008 \\
0.54600 \\
0.60000 \\
0.66000 \\
0.71000 \\
0.76000 \\
0.0 \\
0.96000 \\
0.18000 \\
0.27000 \\
0.35000 \\
0.10000 \\
0.16000 \\
0.52000 \\
0.59000\end{array}$ \\
\hline
\end{tabular}




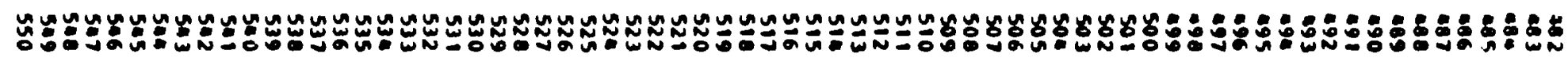

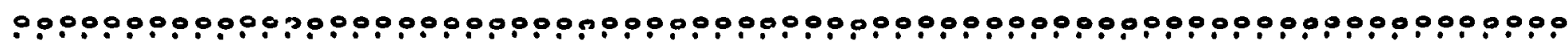

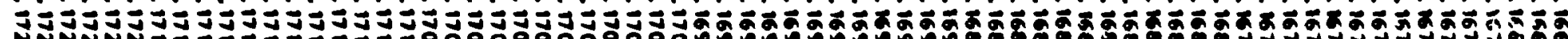

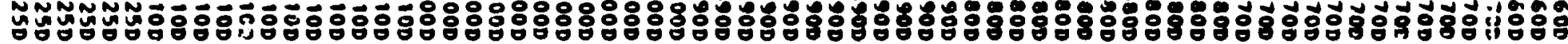

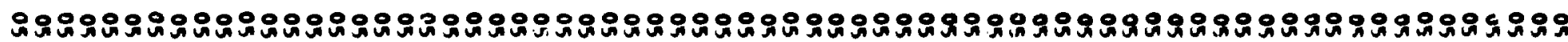

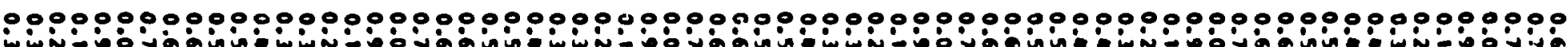

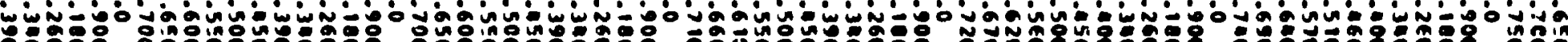

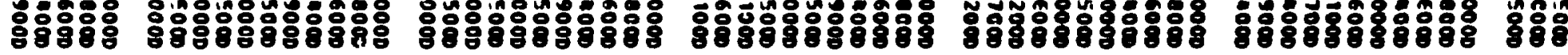

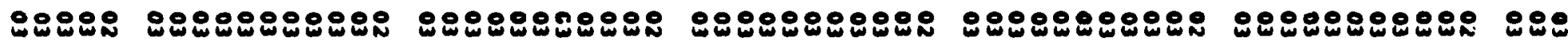




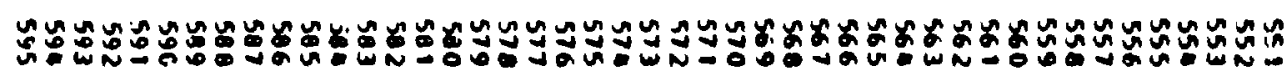

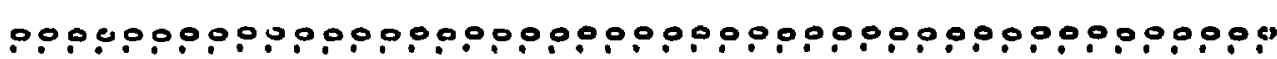

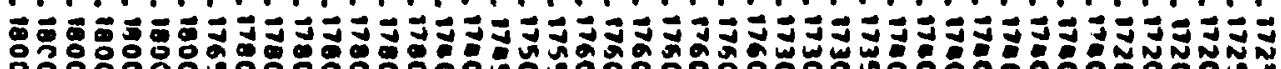

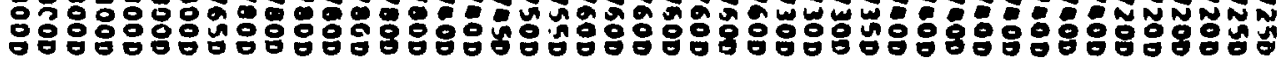

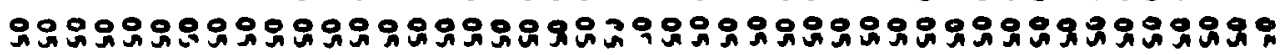

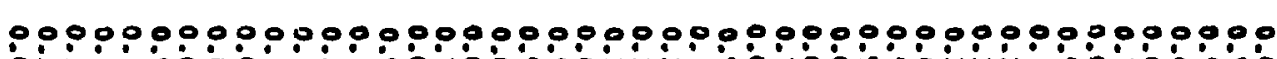
药

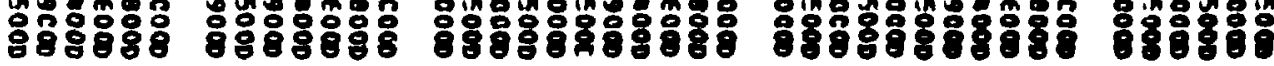

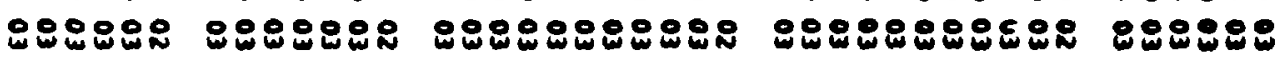




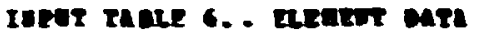

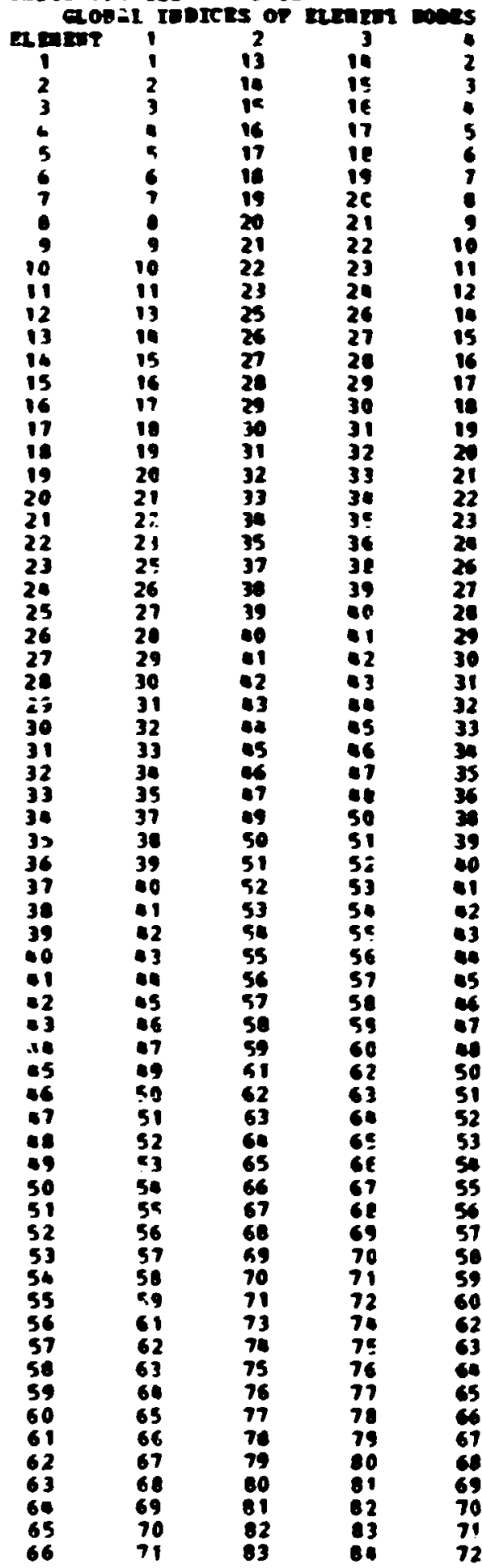

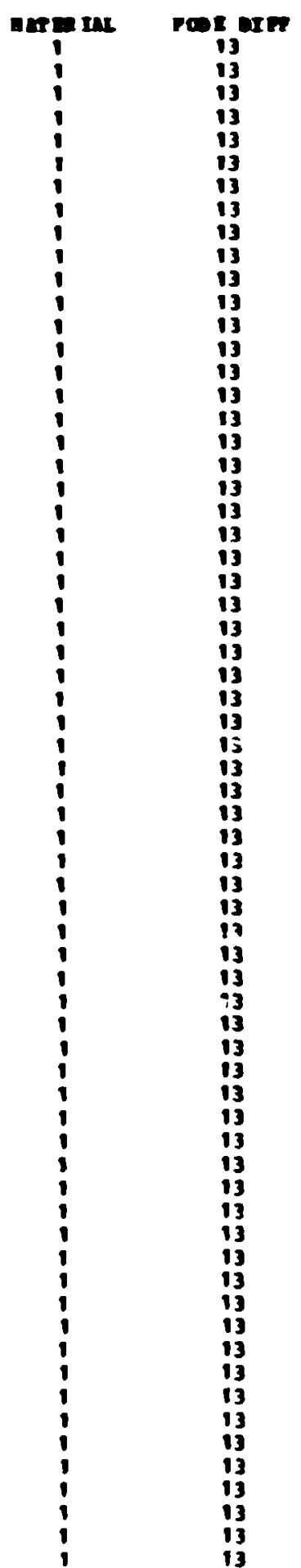




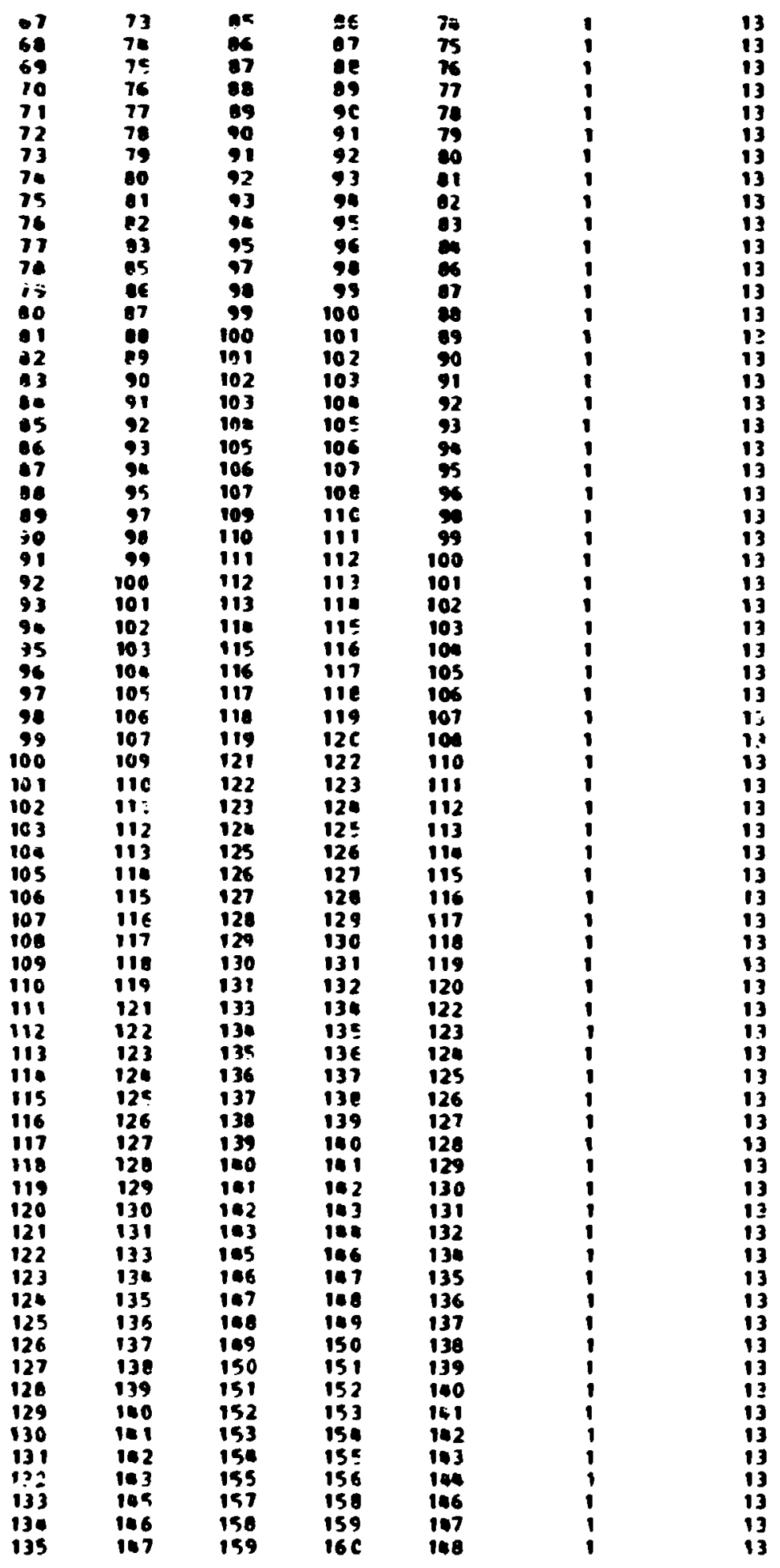




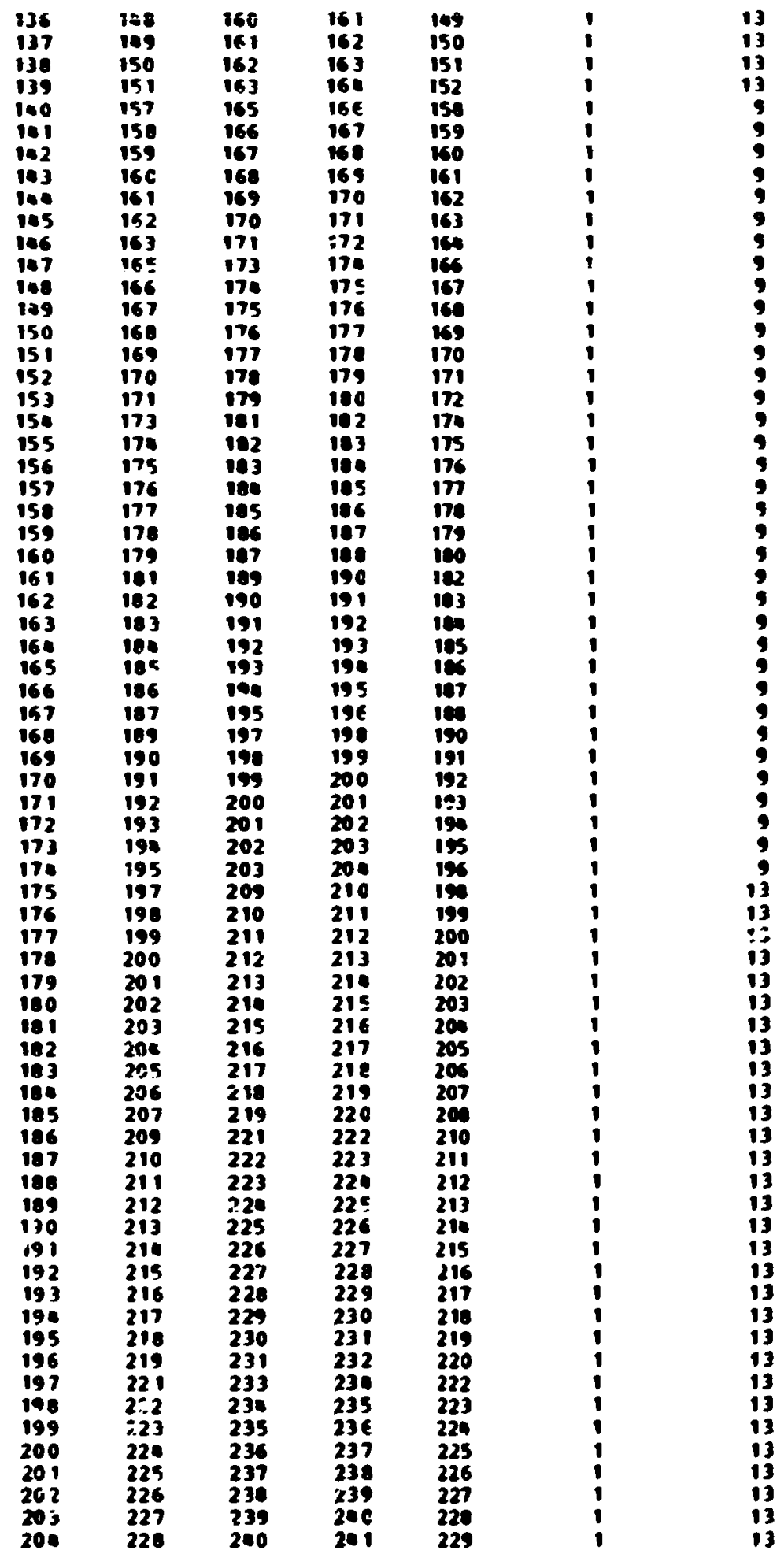




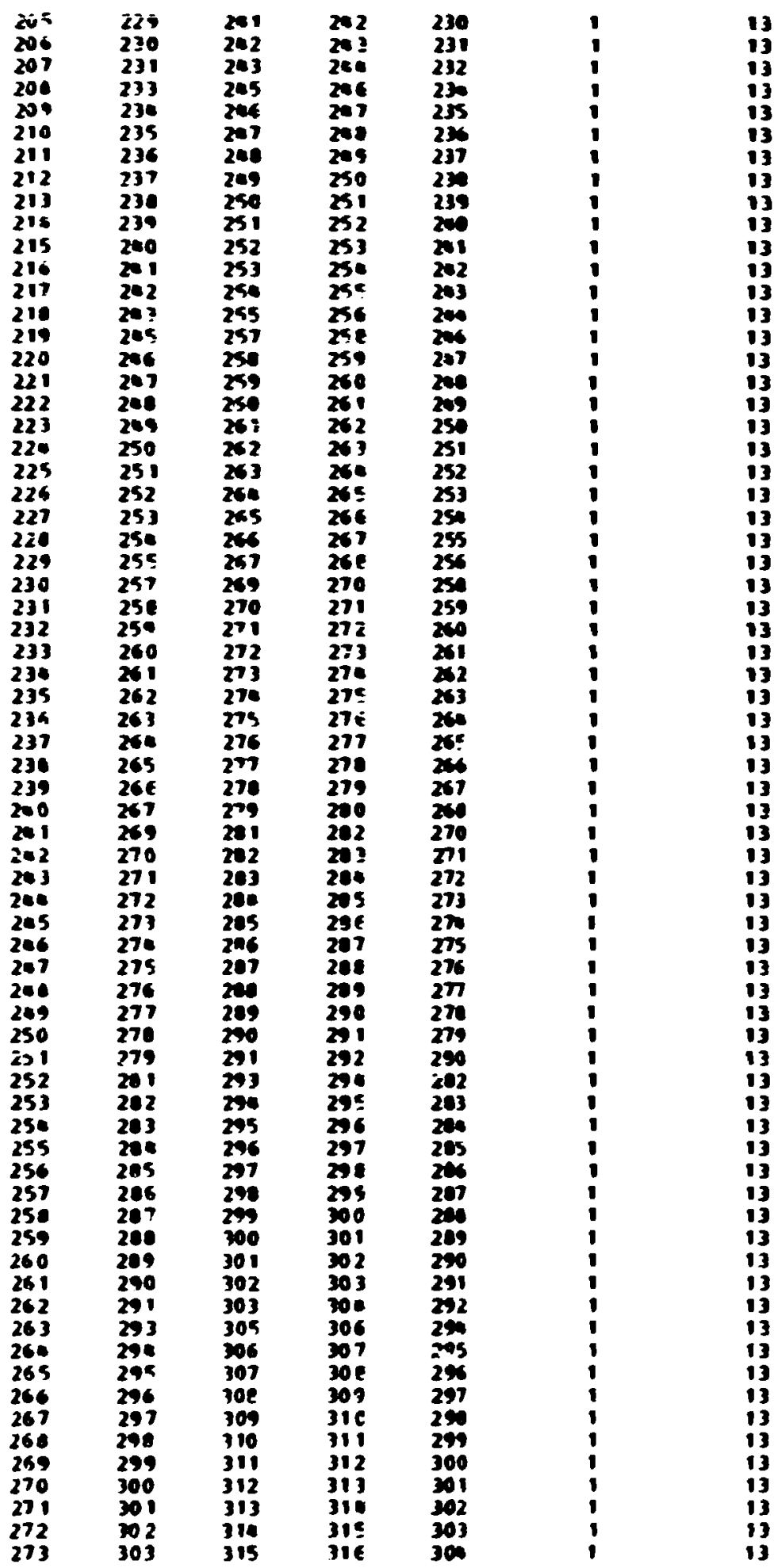




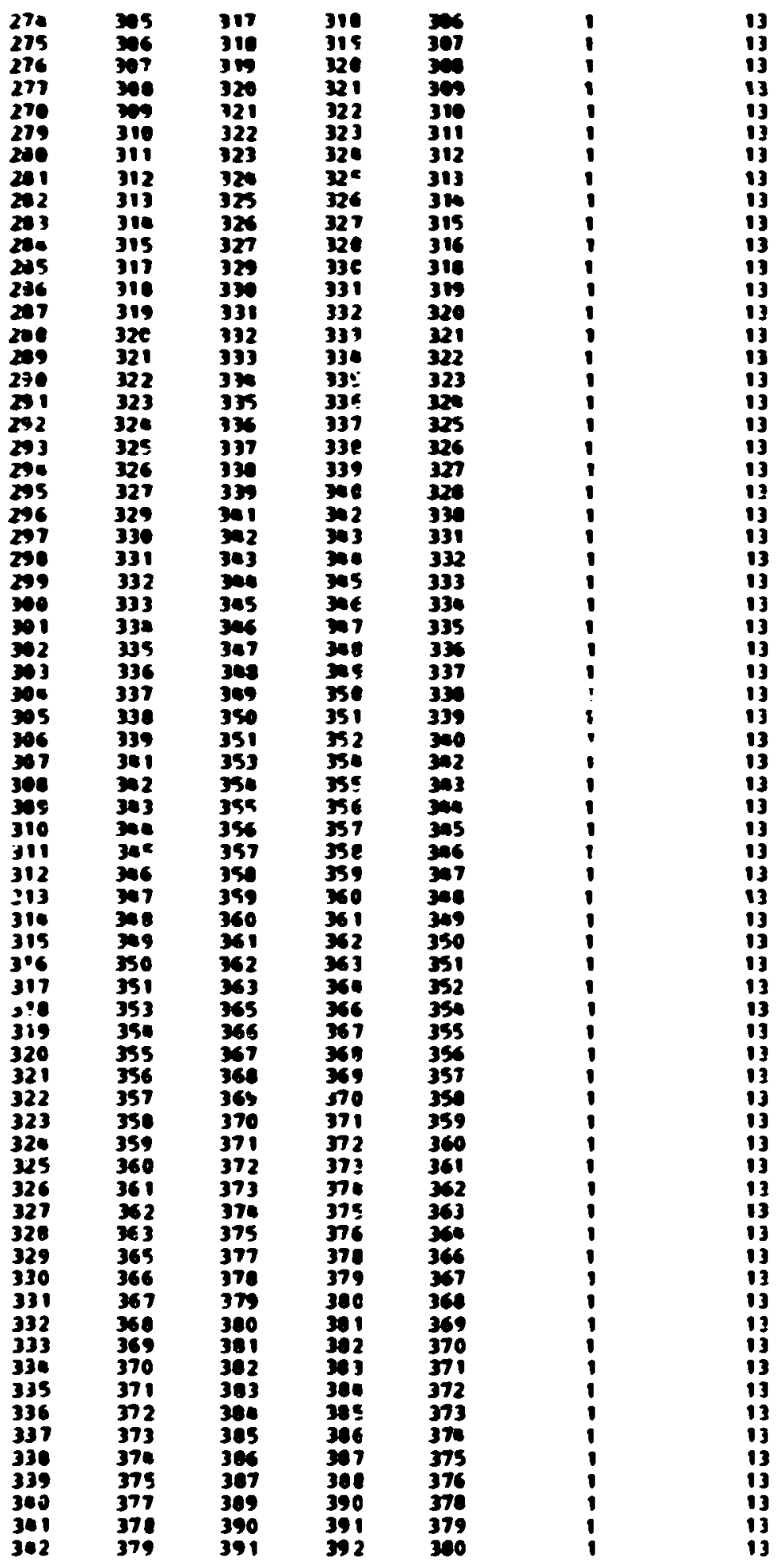




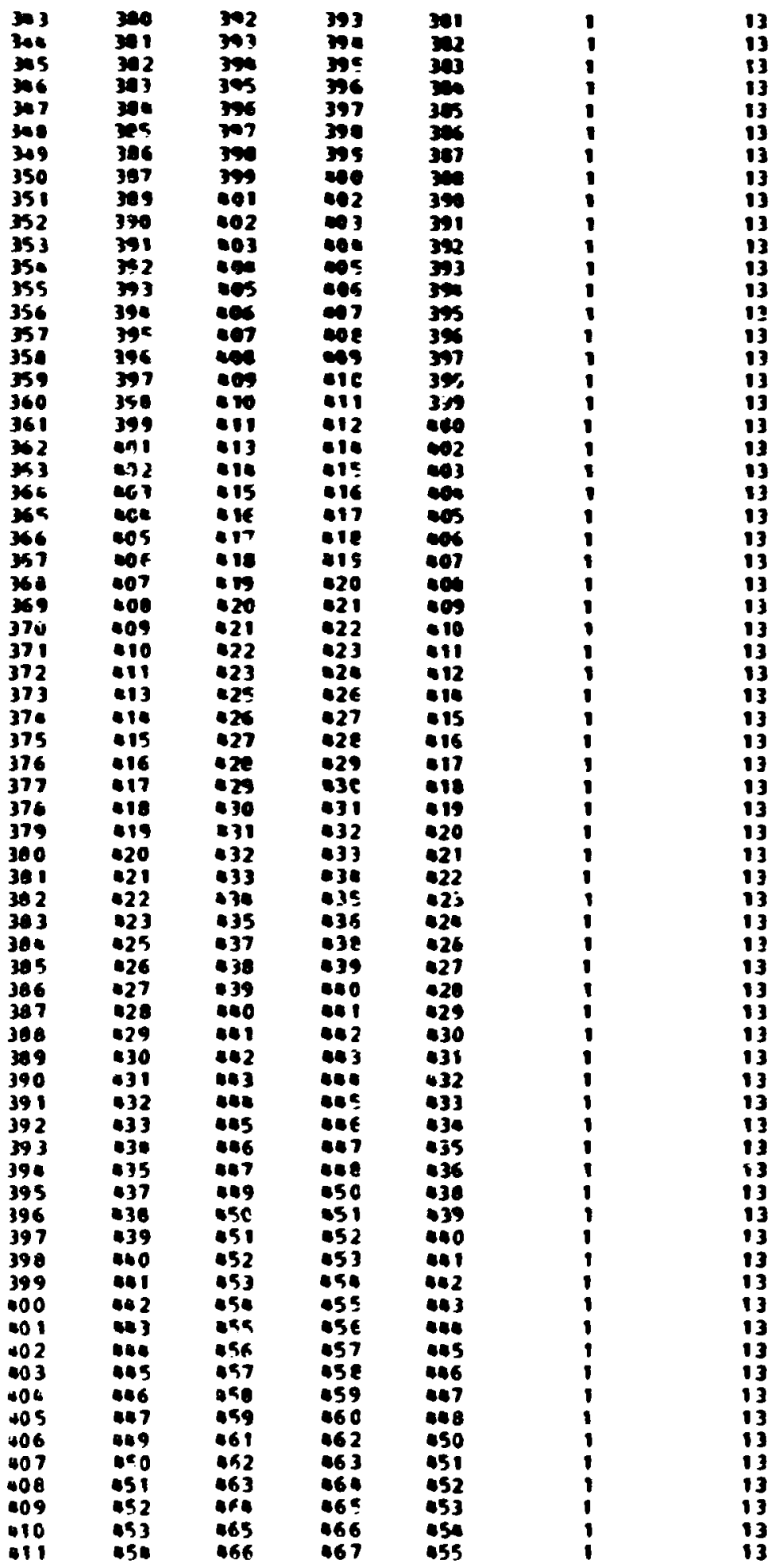




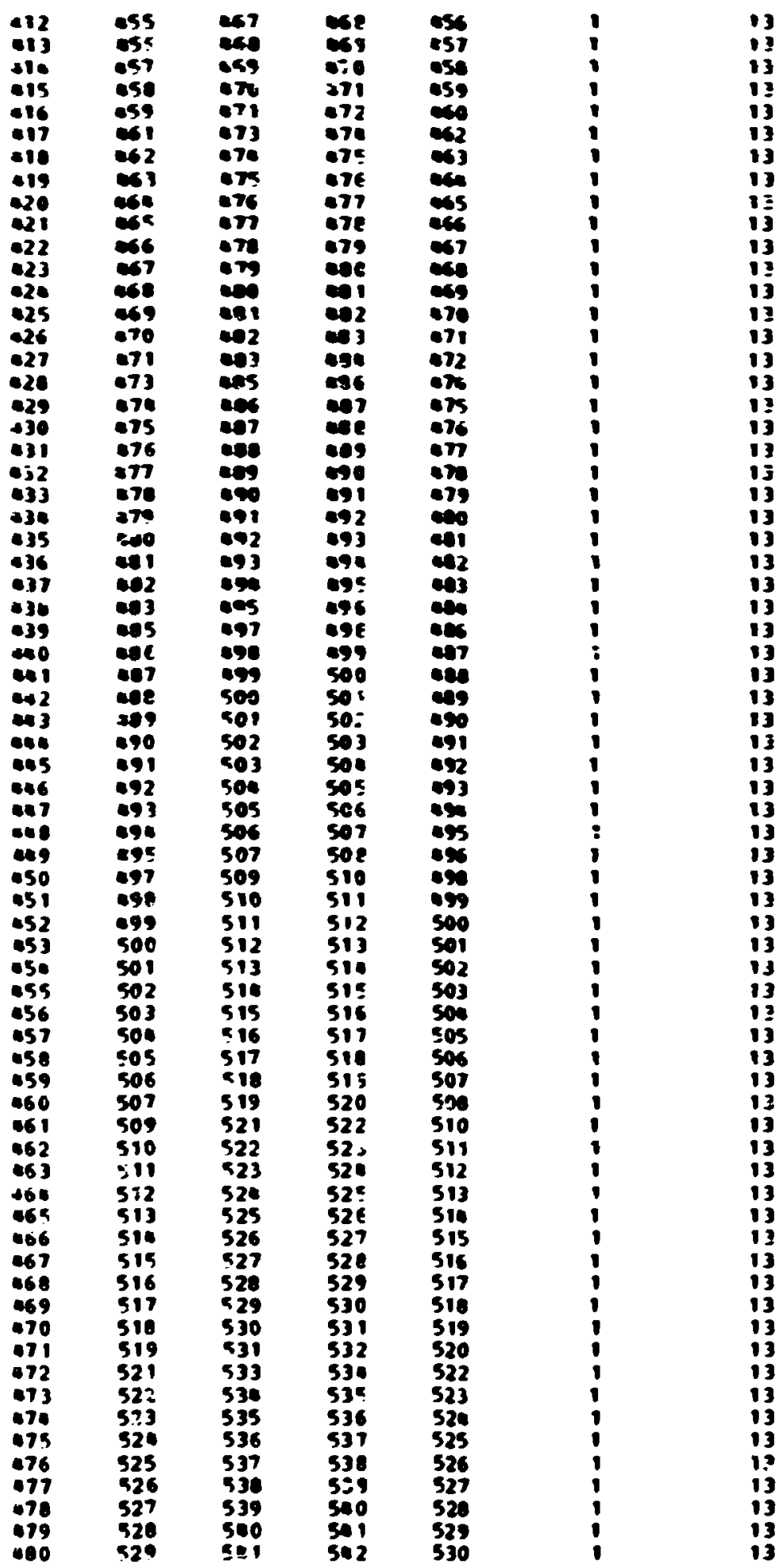




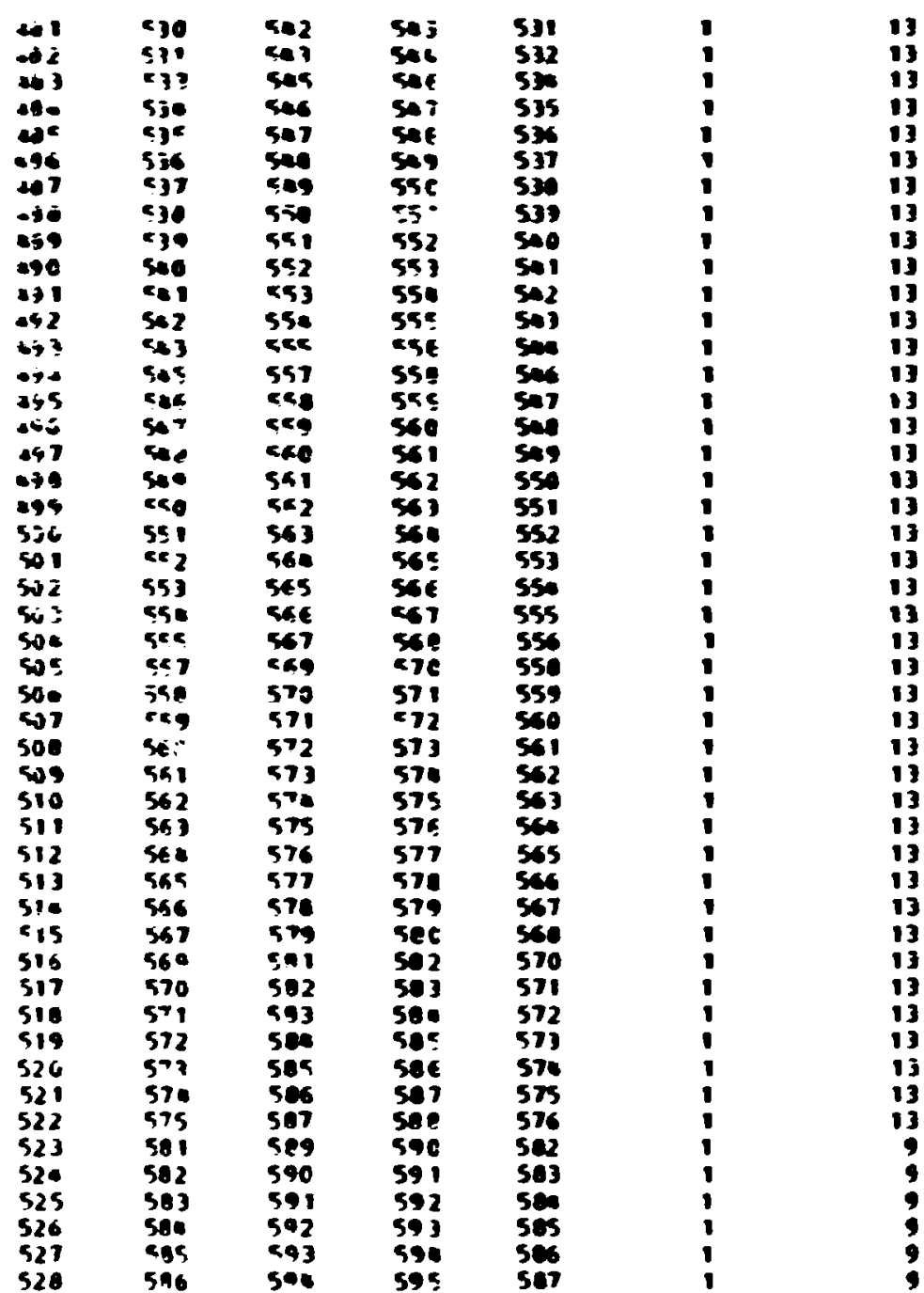




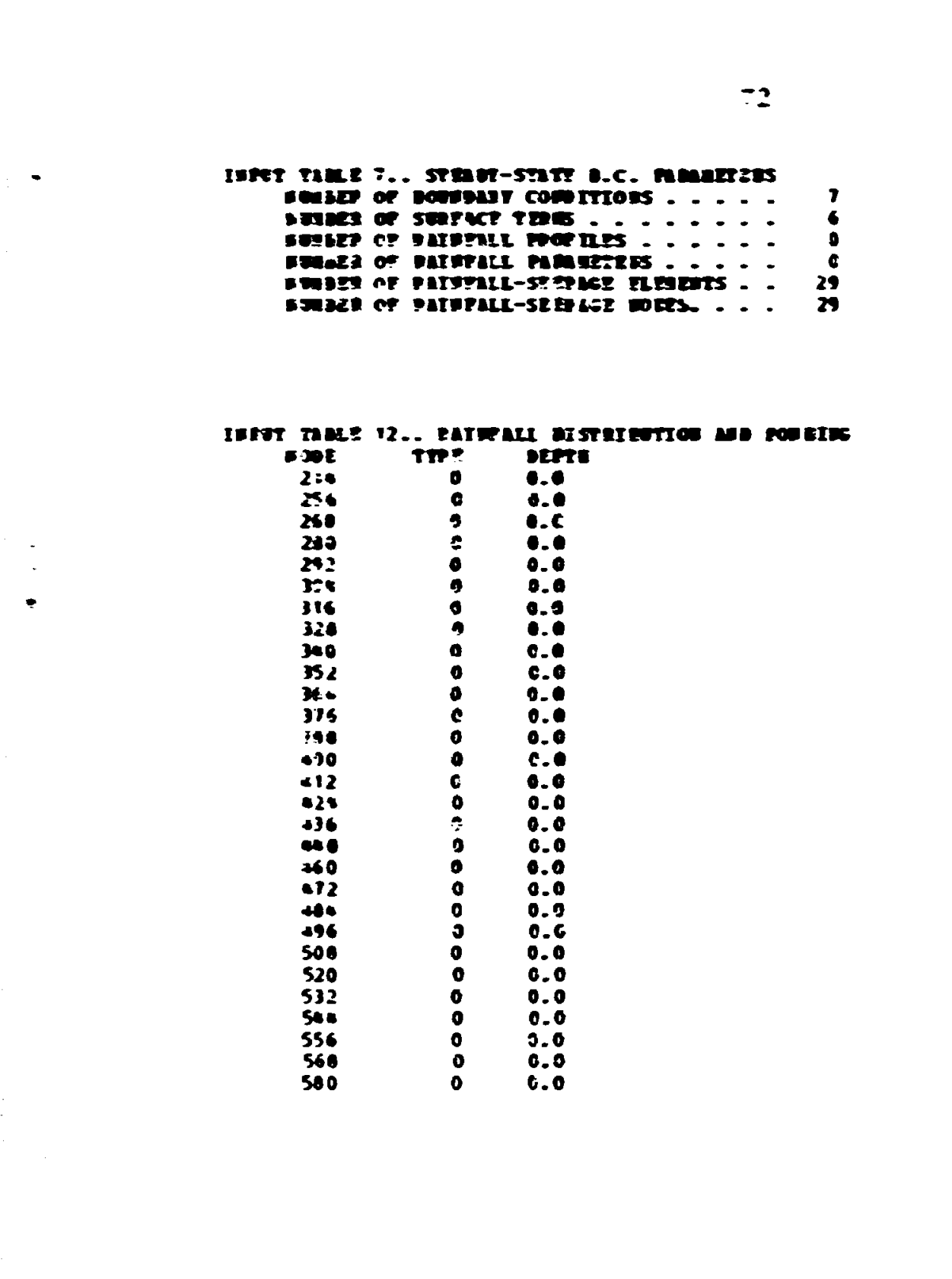




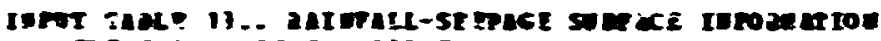

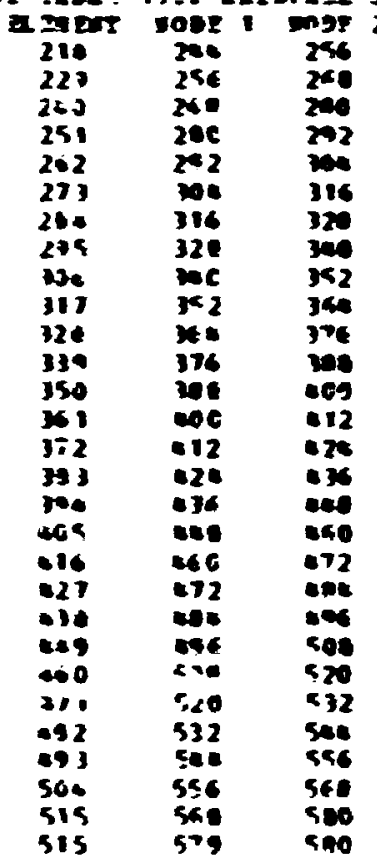

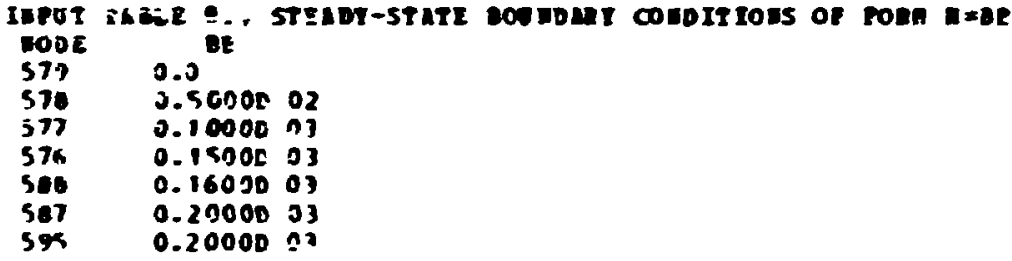

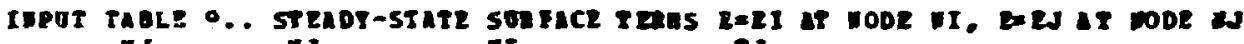

\begin{tabular}{|c|c|c|c|}
\hline $\begin{array}{l}11 \\
152 \\
161 \\
172 \\
160 \\
188\end{array}$ & $\begin{array}{l}10 \\
164 \\
172 \\
190 \\
109 \\
195 \\
290\end{array}$ & $\begin{array}{c}E I \\
-4.50000-00 \\
-4.00000-00 \\
-4.00000-00 \\
-. .00000-00 \\
-4.00000-04 \\
-4.00000-0\end{array}$ & $\begin{array}{l}2 J \\
-6.0000 D-00 \\
-6.00000-04 \\
-6.00000-04 \\
-6.00002-00 \\
-4.0000 D-04 \\
-6.00000-00\end{array}$ \\
\hline
\end{tabular}


00000000000000000000000000000000000000

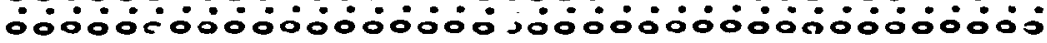

$0000000000000000000000000000000000000 n$

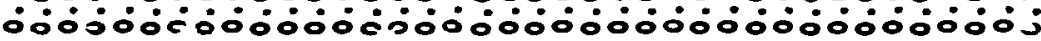

00000000000000000100000000000010000000

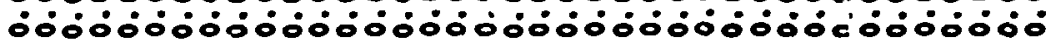

00000000000000000000000000000000000000

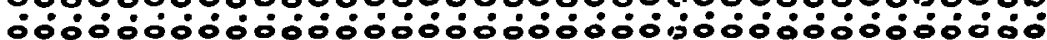

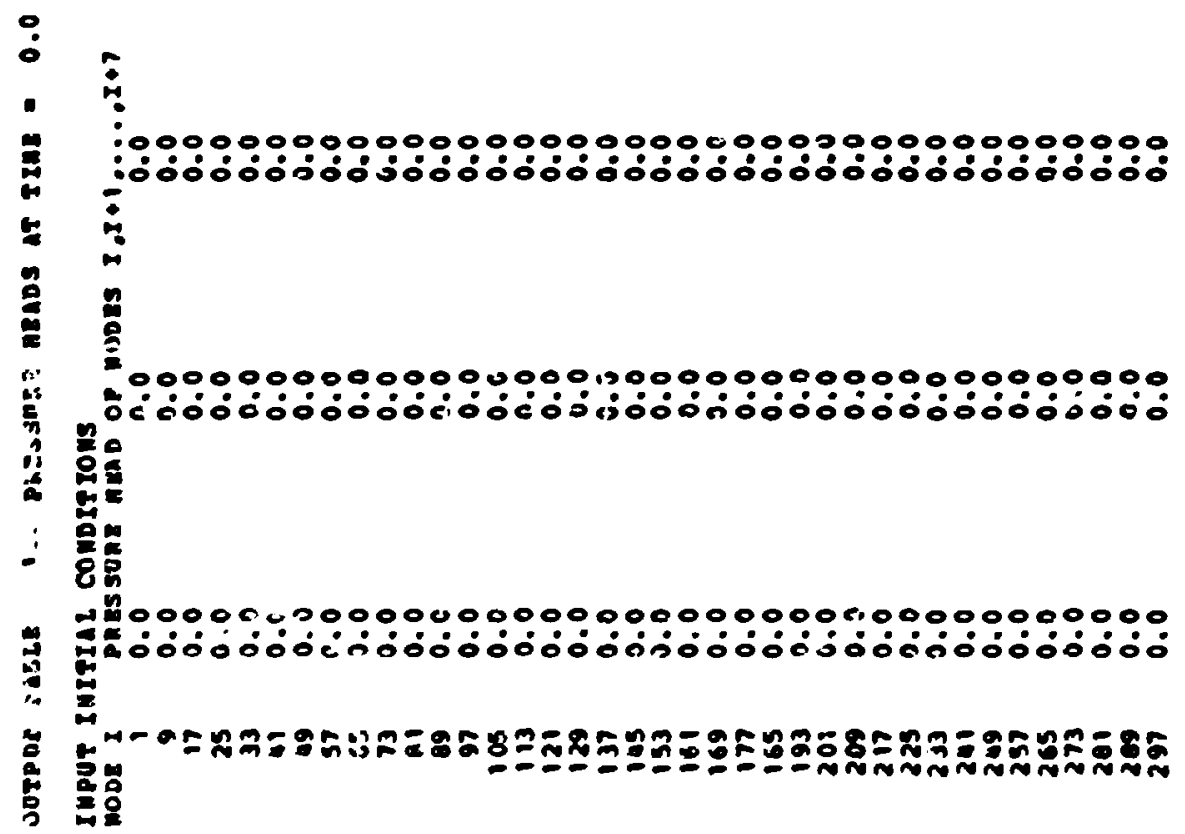


00000000000000000000000000000000000

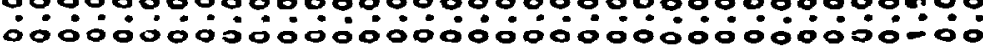

030090000000030000000000000000000000

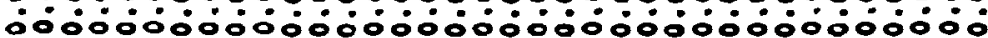
200000002000000000000000000000000000

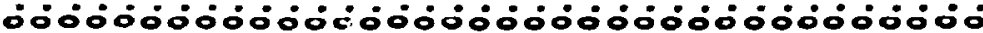

000000000000000000000000000000000000

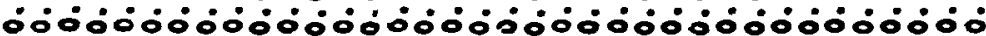

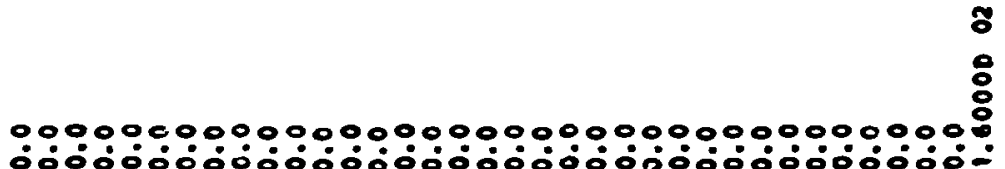
0000000000000000000000000000000000088

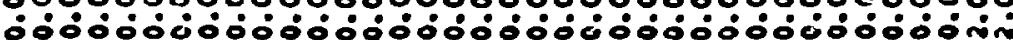

0000000000000000000300000000000000800

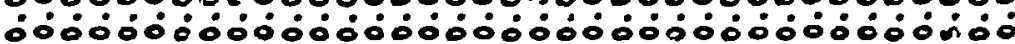

:

8

0000000000000000000000000000040000800

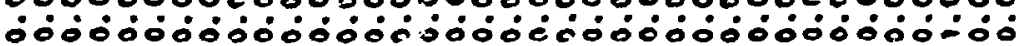

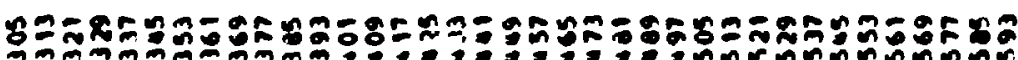




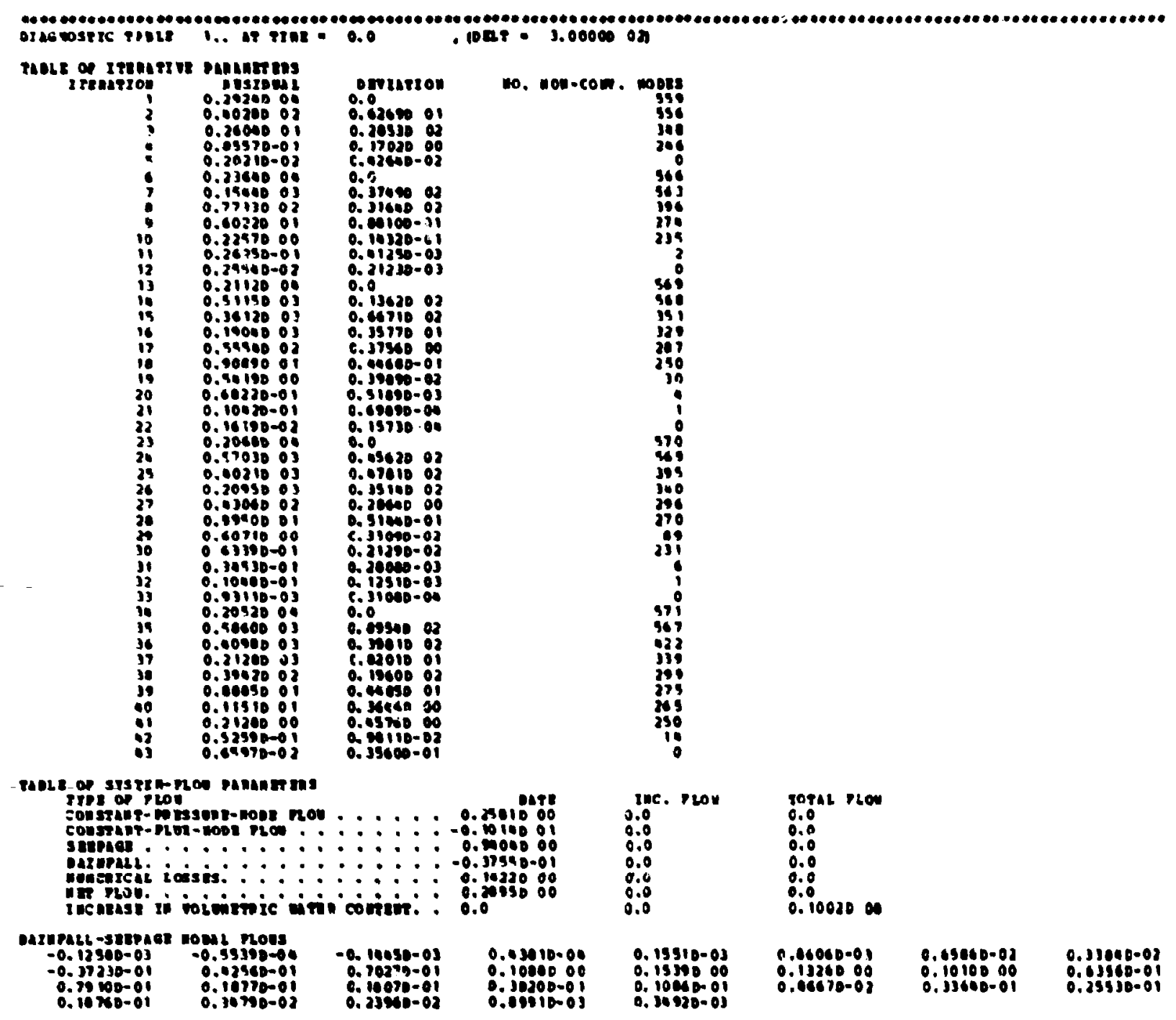




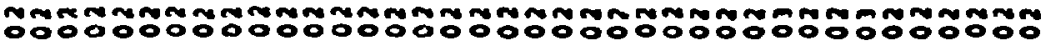

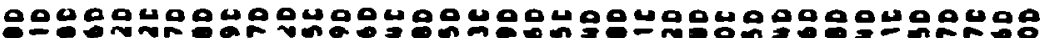

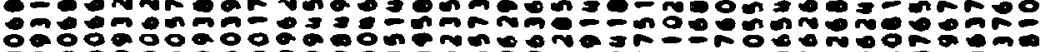

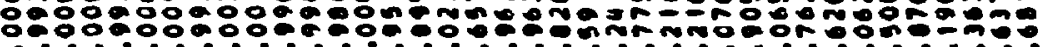

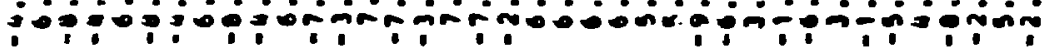

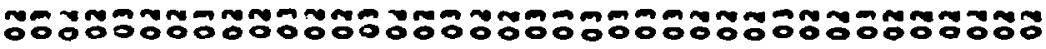
00000000000900000000000000000000000000

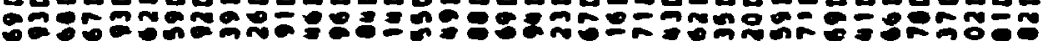

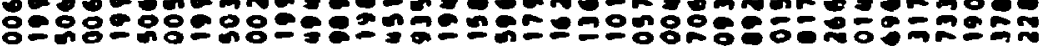

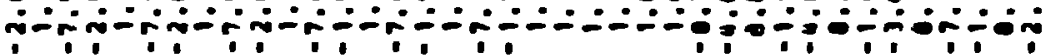

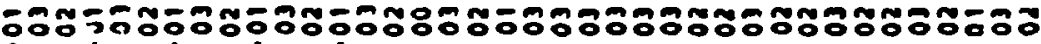

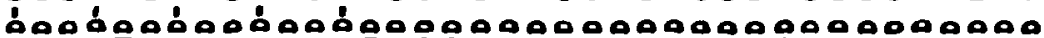

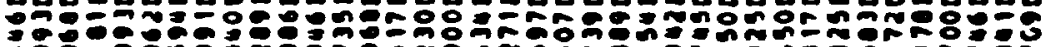

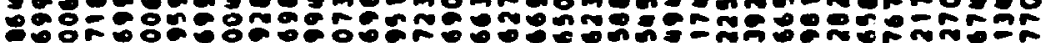

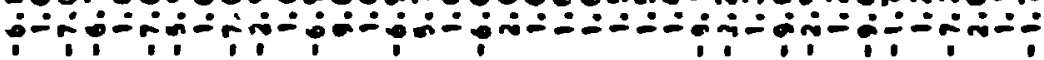

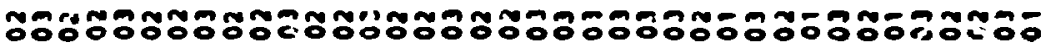
00030000000000000070000000000000000000

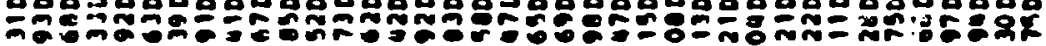

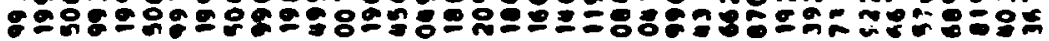

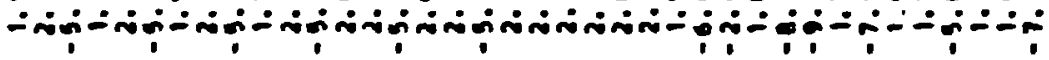

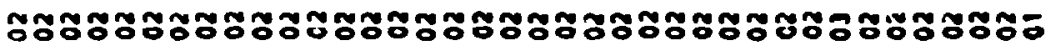

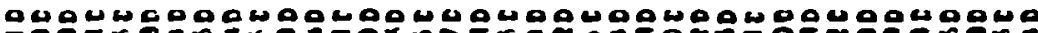

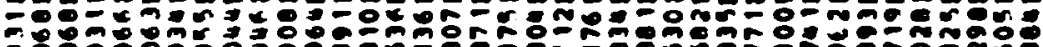

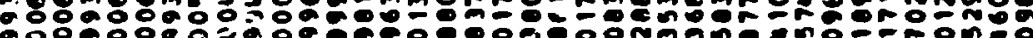

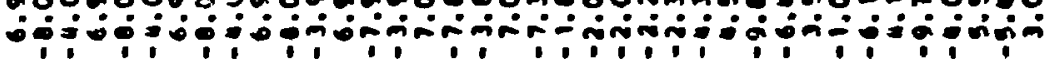

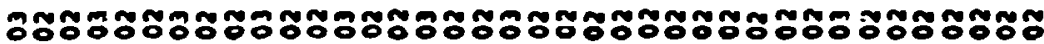
†

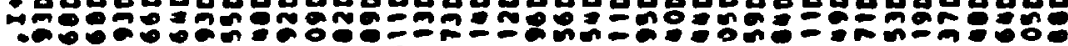

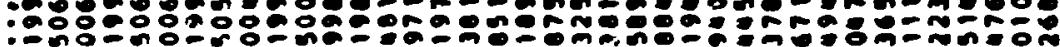

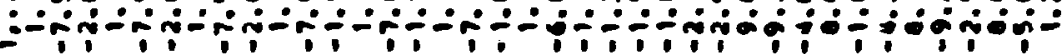
(-

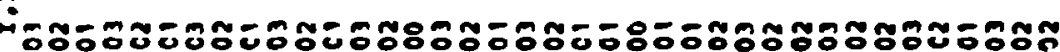

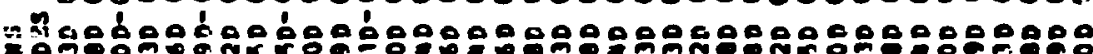

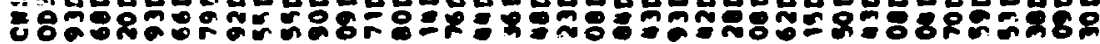

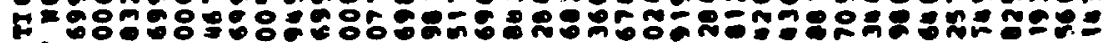

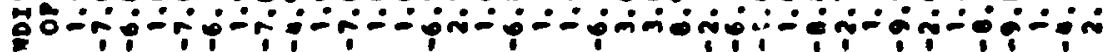
용

(2)

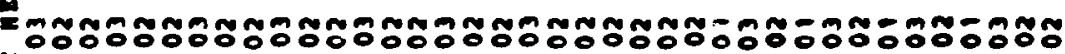
F"

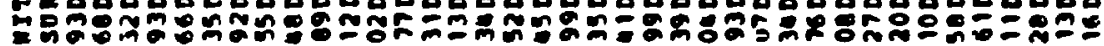

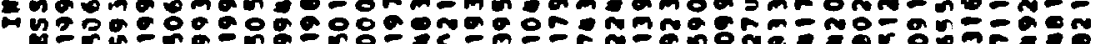

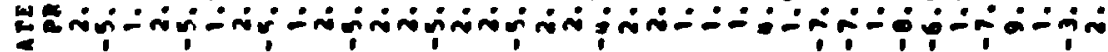
is

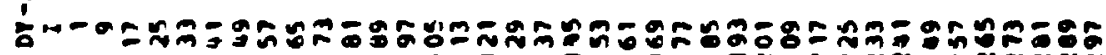
急

点要 


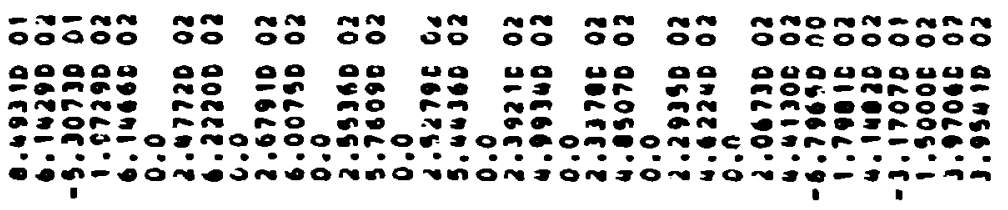

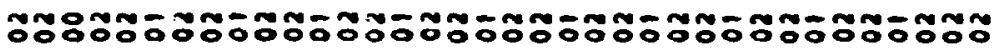

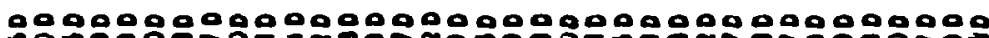

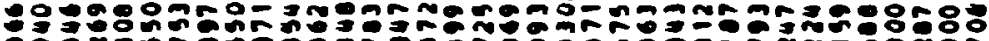

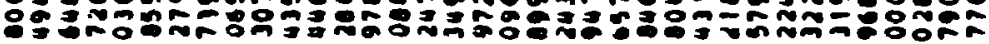

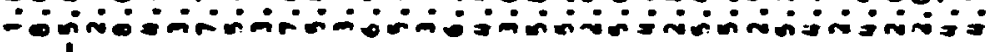

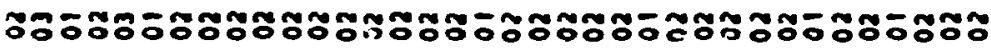

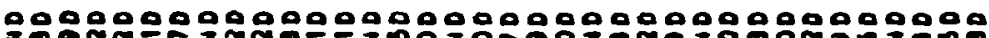

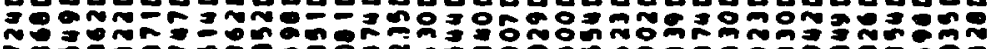

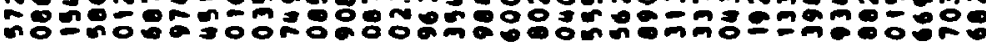

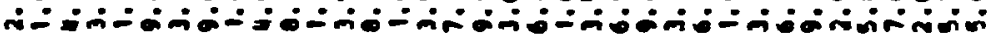

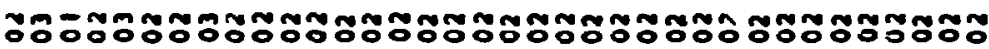

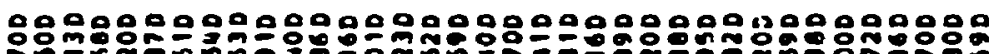

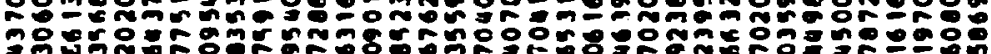

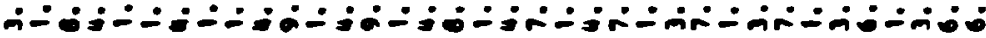

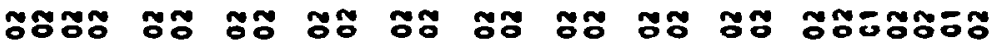

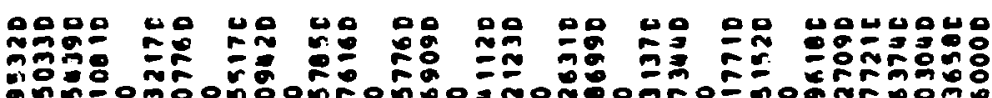

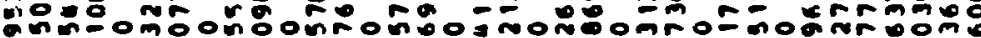

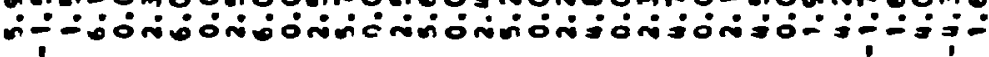

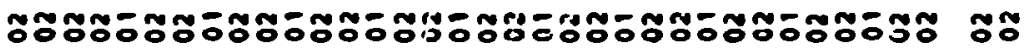

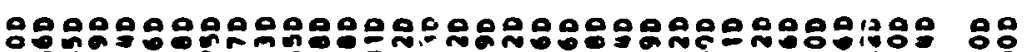

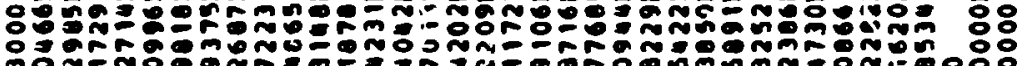

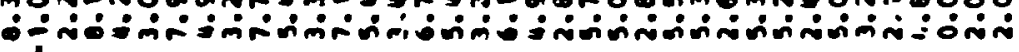

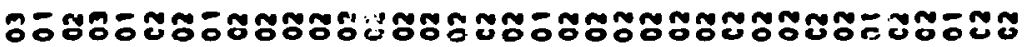

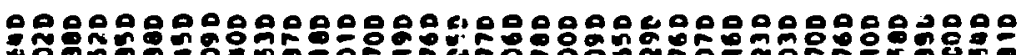

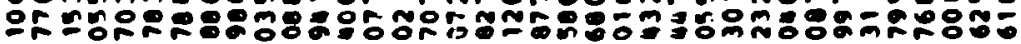

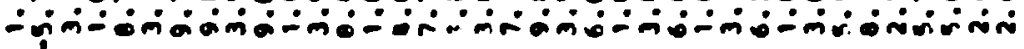

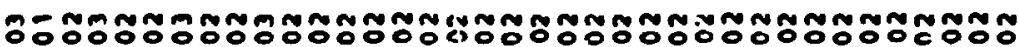

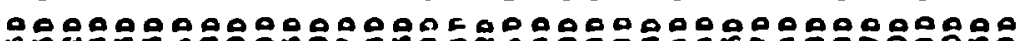

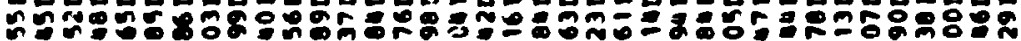

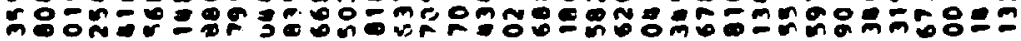

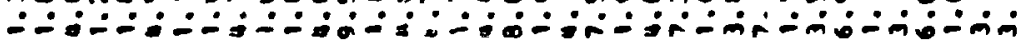

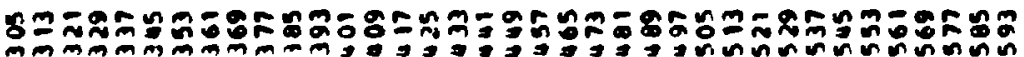


APPENDLX B

INPUT AND OUTPUT FOR SEEPAGE-PIT DISSOLVED-CONSTITUENT TRANSPORT 
INPUT 


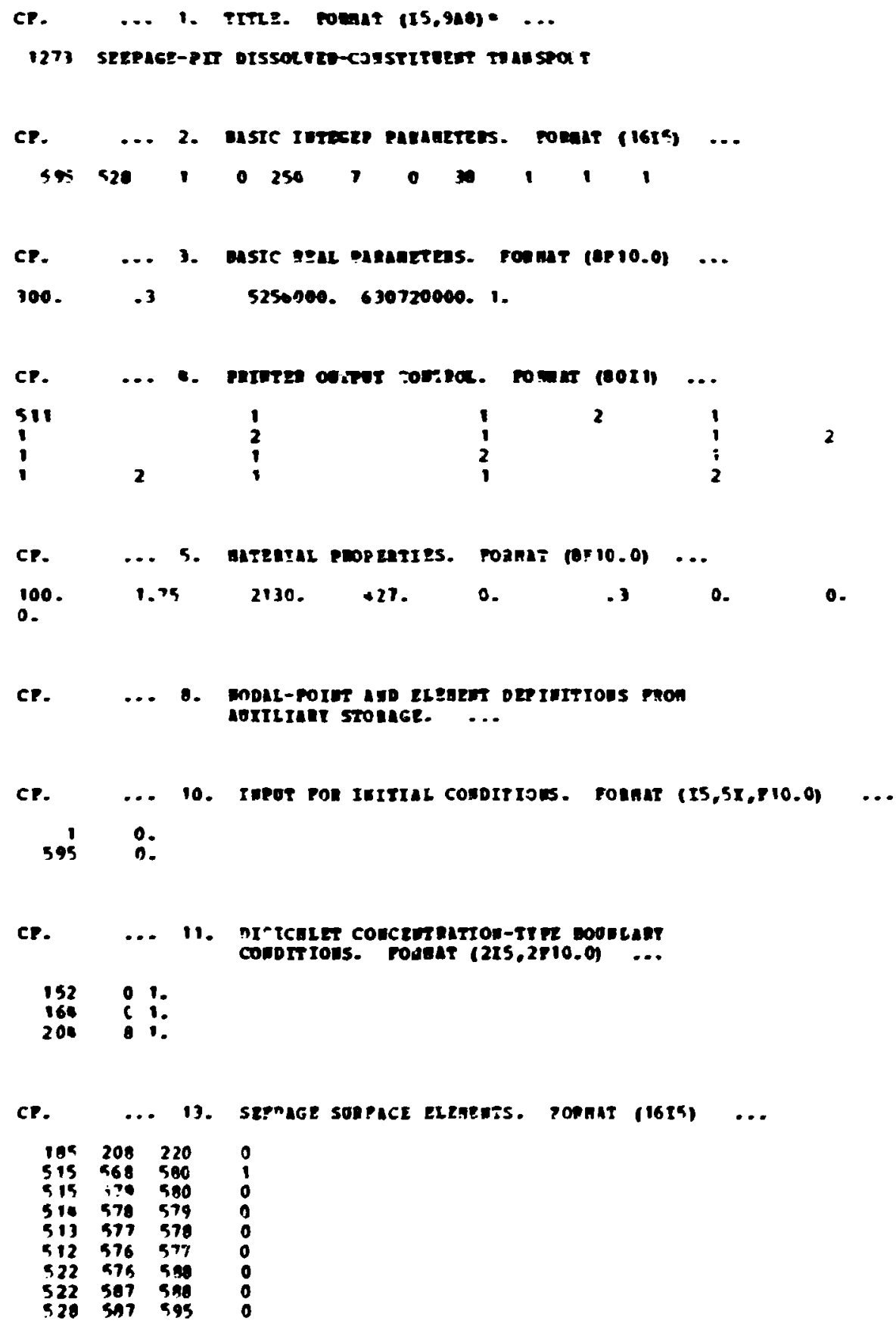

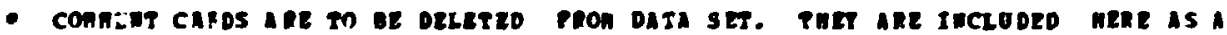
rhoss nepenence ro ipptendx 
OUTPUT

$\prod^{3}$ 


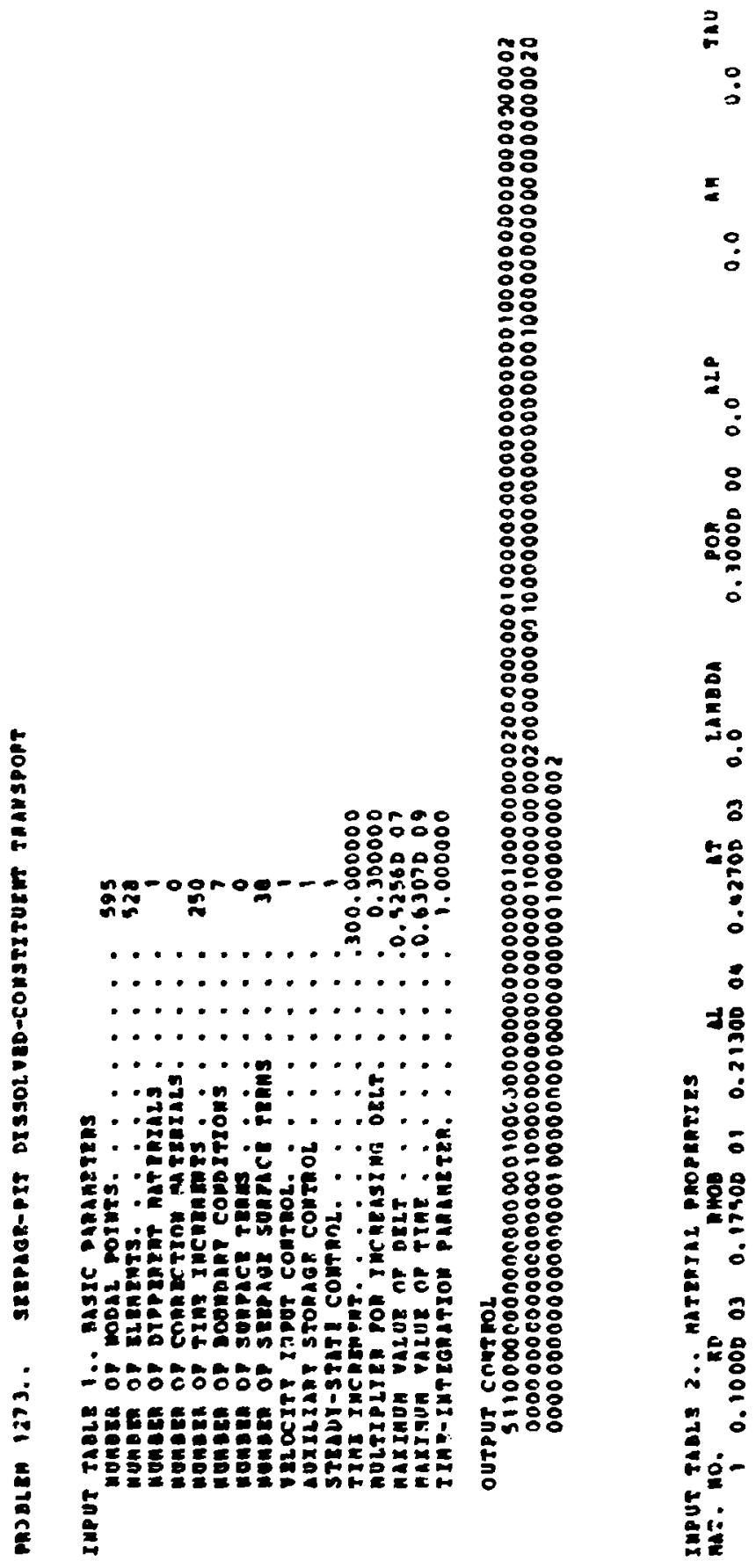




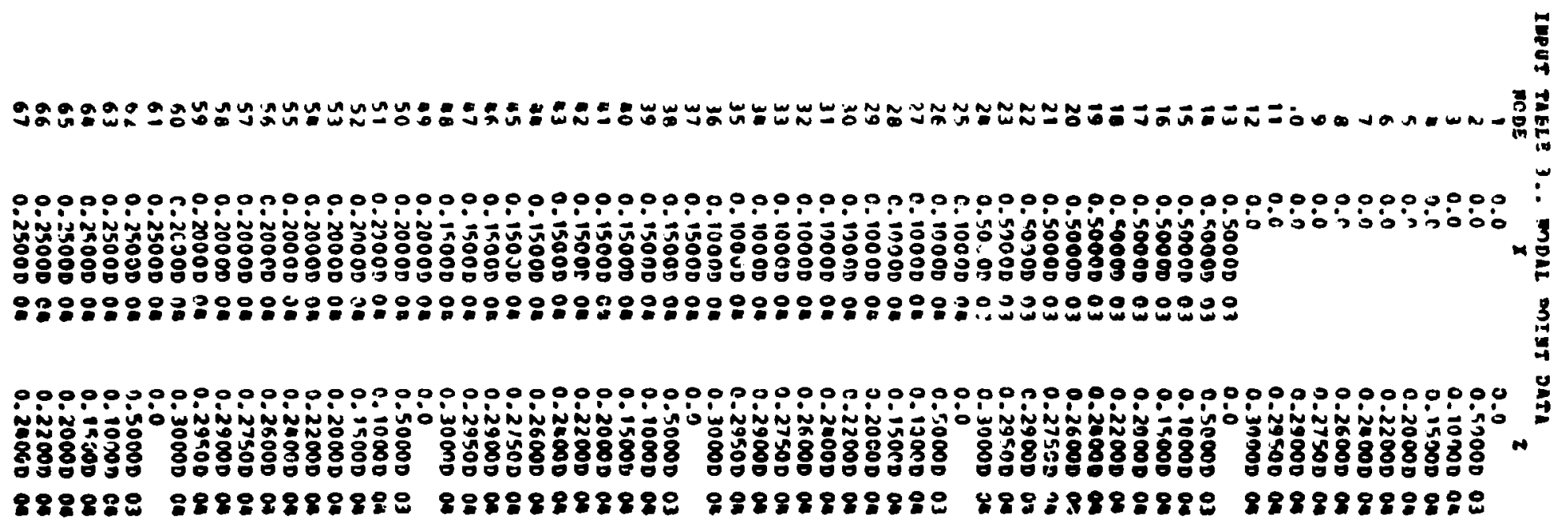




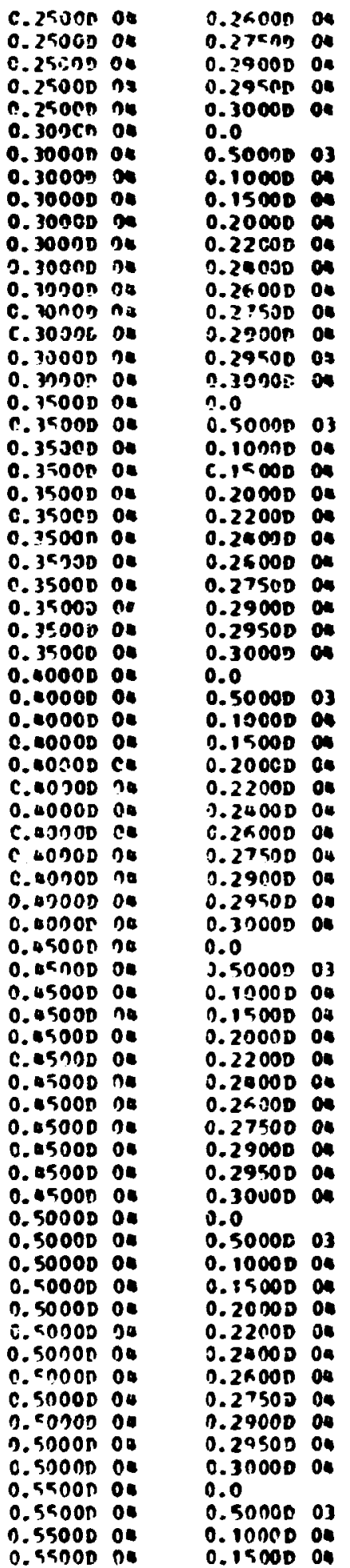




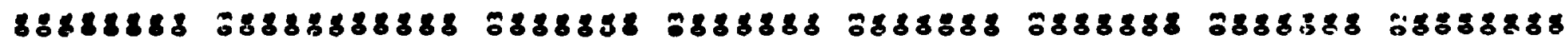

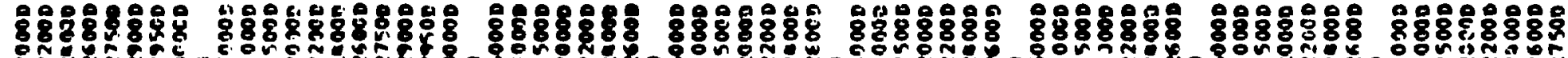

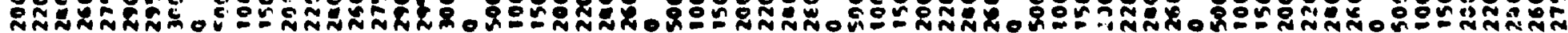

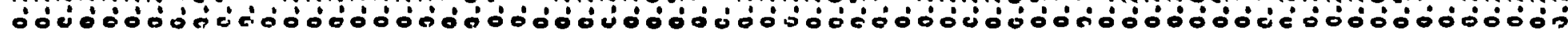

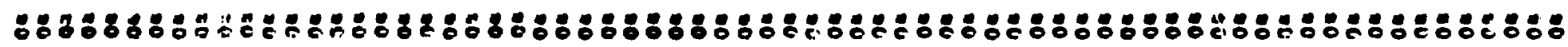

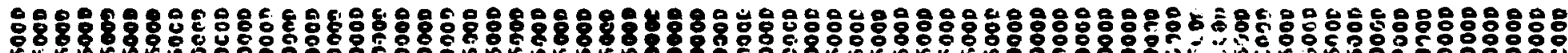

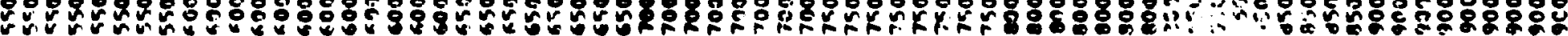

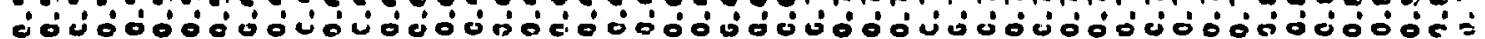

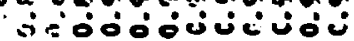

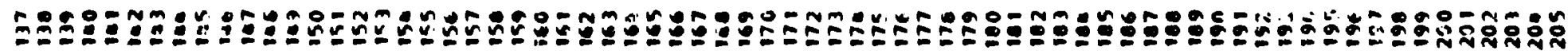




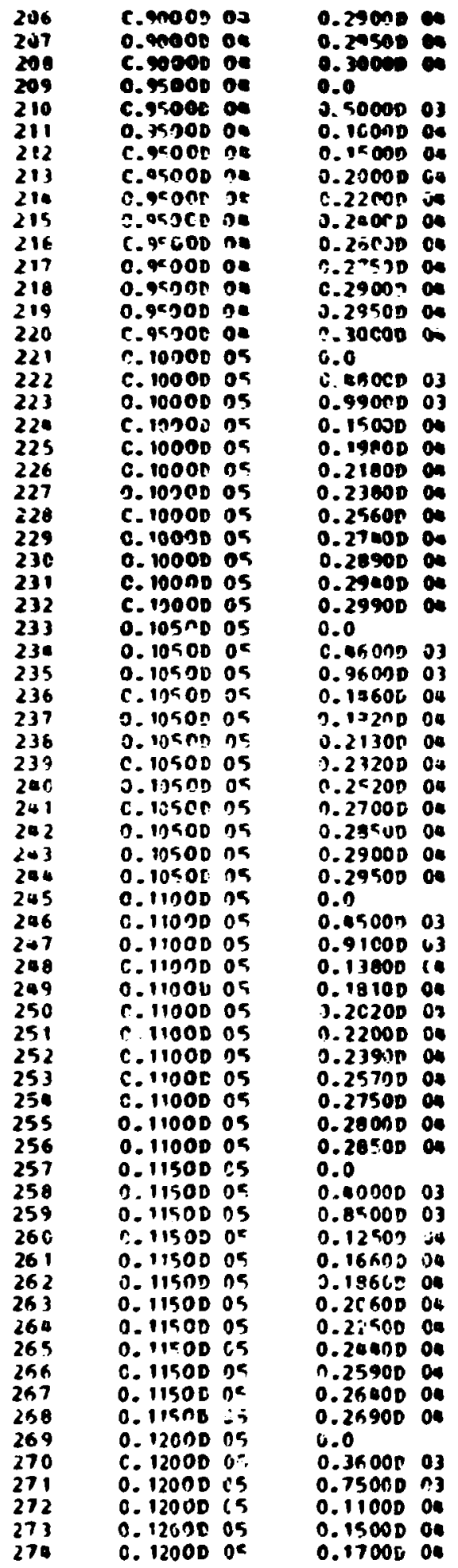




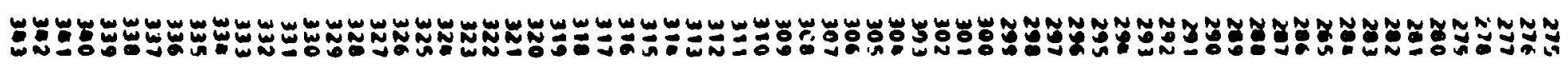



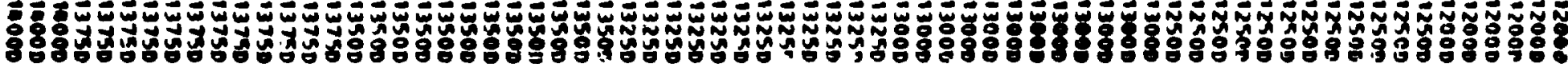

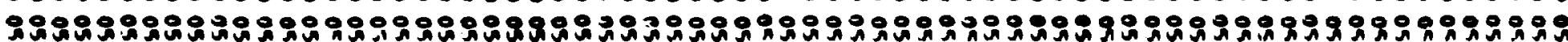

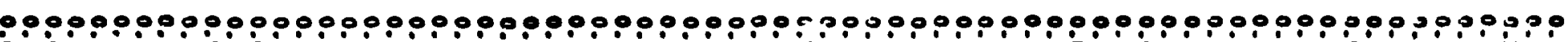

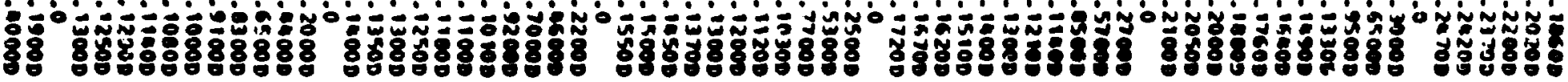

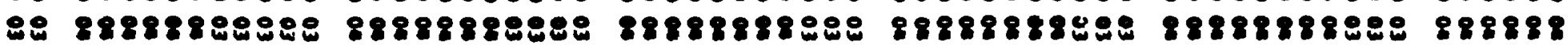




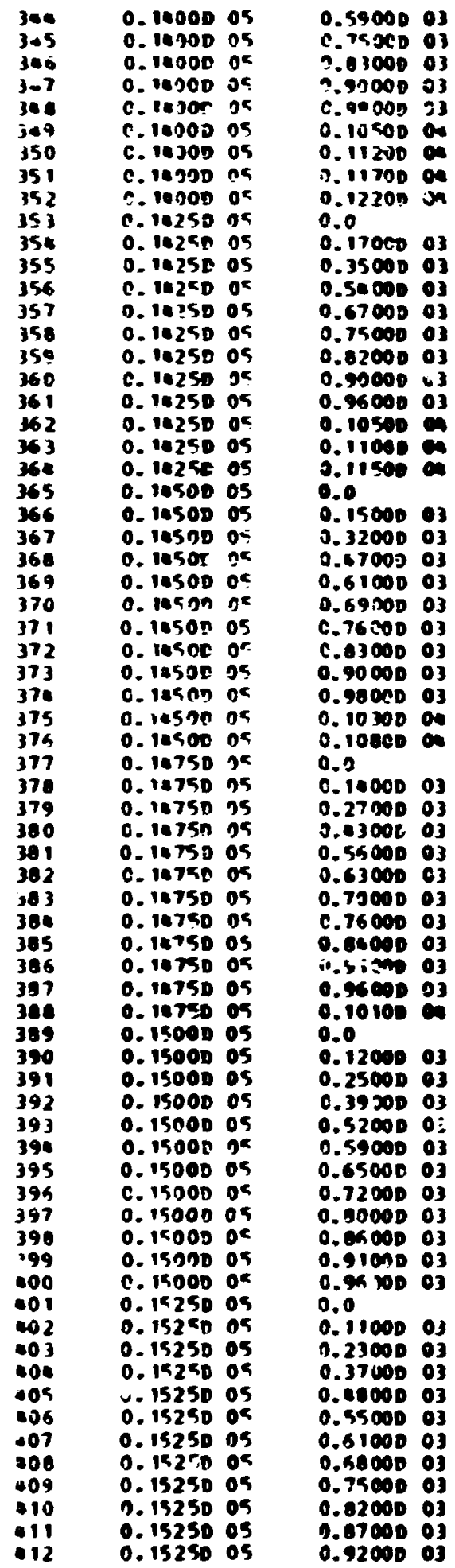




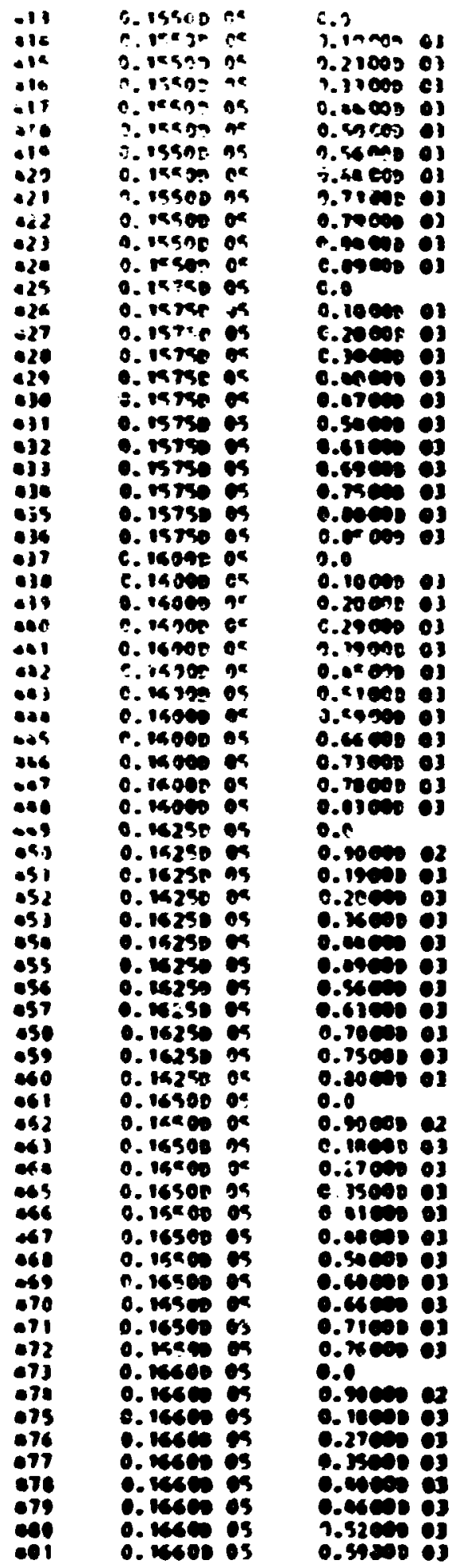




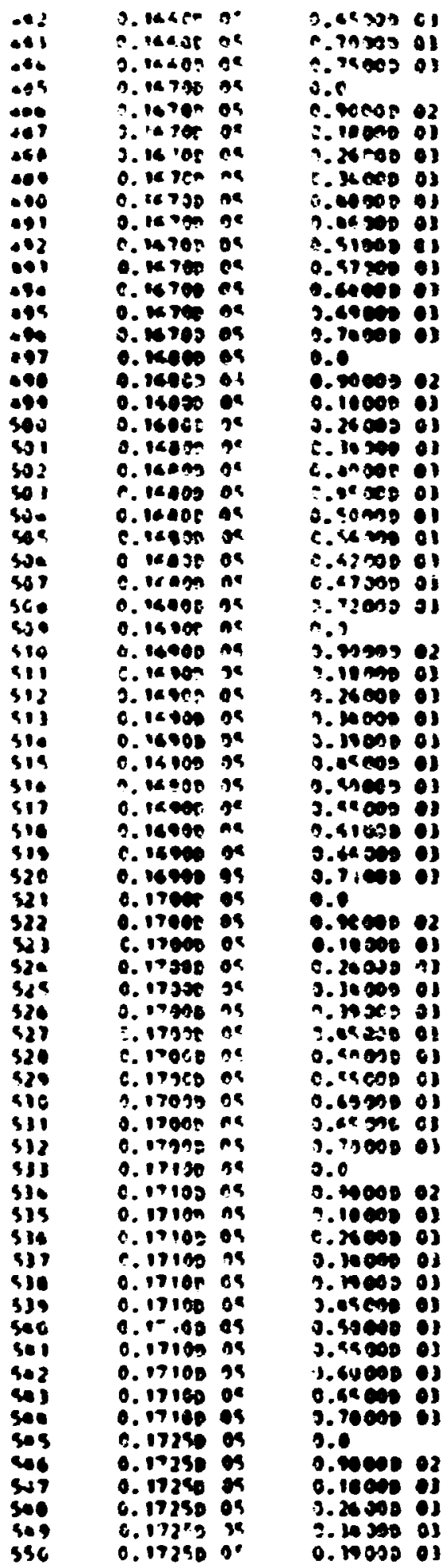




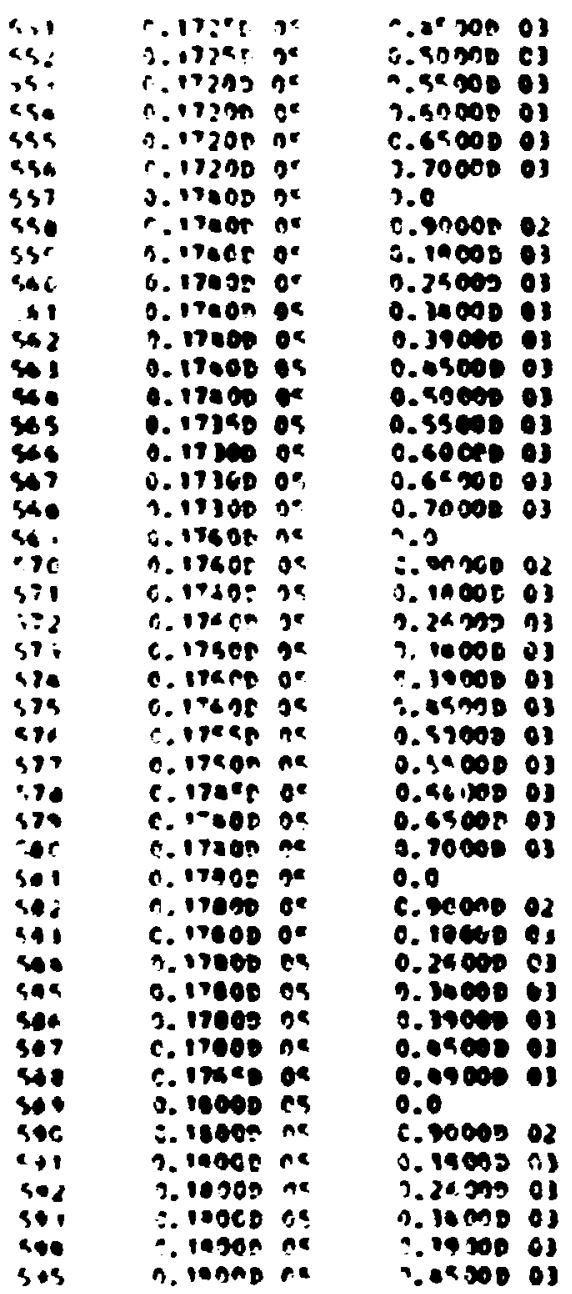




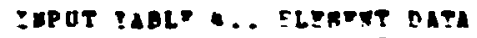

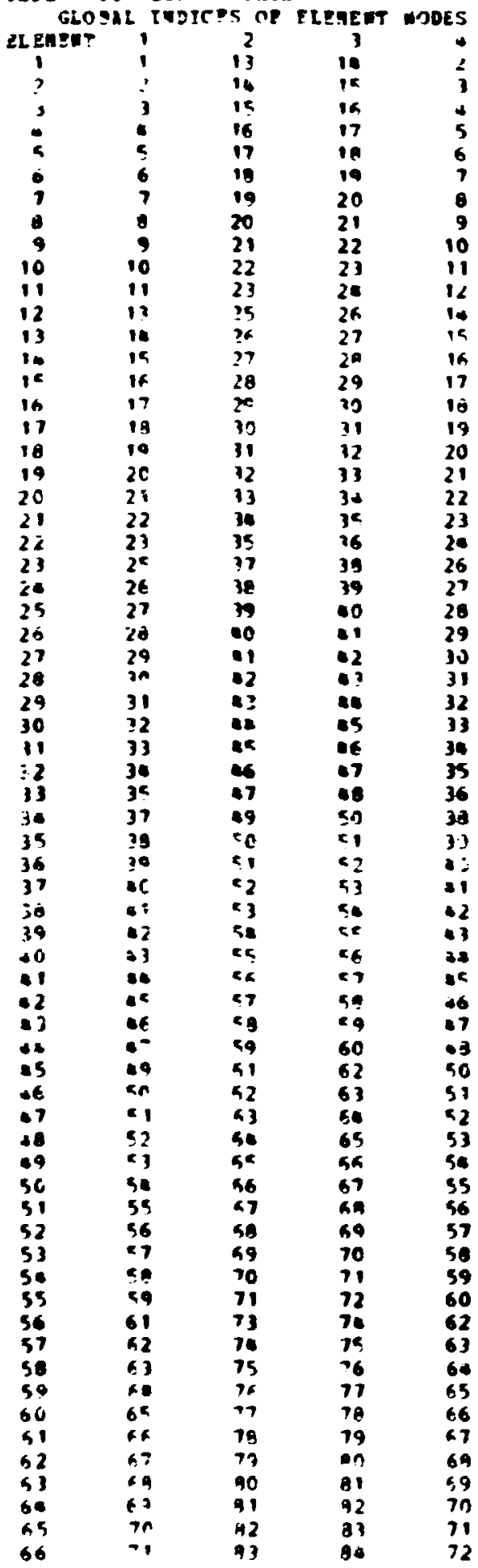

\begin{tabular}{|c|c|}
\hline nAT L्रILL & $\begin{array}{c}\text { DODE DIPY. } \\
13\end{array}$ \\
\hline 1 & 13 \\
\hline 1 & 13 \\
\hline 1 & 13 \\
\hline 1 & 13 \\
\hline 1 & 13 \\
\hline 1 & 13 \\
\hline 1 & 13 \\
\hline 1 & 13 \\
\hline 1 & 13 \\
\hline 1 & 13 \\
\hline 1 & 13 \\
\hline 1 & 13 \\
\hline 1 & 11 \\
\hline 1 & 13 \\
\hline 1 & 13 \\
\hline 1 & 13 \\
\hline 1 & 13 \\
\hline 1 & 13 \\
\hline 1 & 13 \\
\hline 1 & 13 \\
\hline 1 & 13 \\
\hline $\mathbf{1}$ & 13 \\
\hline 1 & 13 \\
\hline 1 & 13 \\
\hline 1 & 13 \\
\hline 1 & 13 \\
\hline 1 & 11 \\
\hline 1 & 13 \\
\hline 1 & 13 \\
\hline 1 & 13 \\
\hline 1 & 13 \\
\hline 1 & 13 \\
\hline 1 & 13 \\
\hline 8 & 13 \\
\hline 1 & 13 \\
\hline 1 & 13 \\
\hline 1 & 13 \\
\hline i & 13 \\
\hline 1 & 13 \\
\hline 1 & 13 \\
\hline 1 & 13 \\
\hline 1 & 13 \\
\hline 1 & 13 \\
\hline 1 & 13 \\
\hline 1 & 13 \\
\hline 1 & 13 \\
\hline 1 & 13 \\
\hline 1 & $\because$ \\
\hline 1 & 13 \\
\hline 1 & 13 \\
\hline 1 & 13 \\
\hline 1 & 13 \\
\hline 1 & 13 \\
\hline 1 & 13 \\
\hline 1 & 13 \\
\hline 1 & 13 \\
\hline 1 & 13 \\
\hline 1 & $: 3$ \\
\hline 1 & 13 \\
\hline 1 & 13 \\
\hline 1 & 13 \\
\hline 1 & 13 \\
\hline 1 & 13 \\
\hline 1 & 13 \\
\hline 1 & 13 \\
\hline
\end{tabular}




\begin{tabular}{|c|c|c|c|c|c|c|}
\hline 57 & 73 & 85 & 86 & 70 & 1 & 13 \\
\hline SB & 78 & 96 & 87 & 75 & i & 13 \\
\hline 59 & r & 87 & 99 & 76 & i & 13 \\
\hline 70 & 76 & pe & 99 & 77 & i & 13 \\
\hline$\pi$ & 77 & $\because 9$ & 90 & 78 & : & 13 \\
\hline 72 & 18 & 00 & 91 & 79 & ' & 13 \\
\hline 73 & 70 & 91 & 92 & 80 & 1 & $\begin{array}{l}13 \\
13\end{array}$ \\
\hline is & $\begin{array}{l}80 \\
81\end{array}$ & 92 & 93 & $\begin{array}{l}81 \\
82\end{array}$ & i & $\begin{array}{l}13 \\
13\end{array}$ \\
\hline 76 & & & 93 & 33 & i & \\
\hline$i$ & 83 & 95 & 96 & $\theta$ & 1 & 13 \\
\hline $\begin{array}{l}78 \\
73\end{array}$ & $\begin{array}{l}85 \\
\text { A6 }\end{array}$ & $\begin{array}{l}97 \\
98\end{array}$ & $\begin{array}{l}90 \\
90\end{array}$ & $\begin{array}{l}86 \\
87\end{array}$ & i & $\begin{array}{l}13 \\
13\end{array}$ \\
\hline 80 & & & 190 & 26 & ; & 13 \\
\hline 81 & Ae & 100 & 101 & 69 & 1 & 13 \\
\hline 02 & 69 & 101 & 132 & 90 & ' & 13 \\
\hline 3 & $9 c$ & 102 & 103 & 91 & ! & 13 \\
\hline के & g1 & in3 & Cen & 92 & ; & 13 \\
\hline $\begin{array}{l}25 \\
86\end{array}$ & $\begin{array}{l}92 \\
07\end{array}$ & $\begin{array}{l}\operatorname{lng} \\
\operatorname{lng}\end{array}$ & $\begin{array}{l}1006 \\
\text { ins }\end{array}$ & $\begin{array}{l}53 \\
96\end{array}$ & ! & 13 \\
\hline 37 & 9 & 136 & 197 & 9 & i & 13 \\
\hline 38 & cs & $10^{-}$ & :0n & 96 & I & 13 \\
\hline 89 & 97 & 100 & 110 & 98 & I & 13 \\
\hline 90 & 98 & ic & $\cdots$ & 99 & I & i3 \\
\hline 91 & 97 & 11 & 112 & 100 & ' & 13 \\
\hline$\div 2$ & 100 & 112 & 113 & 101 & I & 13 \\
\hline 33 & 101 & 113 & IIa & 102 & i & 13 \\
\hline 36 & 102 & in & is & 103 & i & ? \\
\hline $9^{-}$ & 103 & 115 & 11 & 100 & i & 13 \\
\hline ge & 104 & 116 & $1 \because 3$ & 105 & : & 13 \\
\hline 47 & 105 & 117 & 119 & 106 & I & 13 \\
\hline 30 & 306 & 118 & 119 & 107 & ; & 13 \\
\hline 99 & 101 & 119 & 120 & 100 & I & 13 \\
\hline 100 & 109 & 121 & 122 & 110 & I & 13 \\
\hline 101 & 110 & 122 & 123 & 111 & I & 13 \\
\hline 102 & 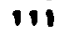 & 123 & 124 & 112 & i & 13 \\
\hline 133 & 112 & 120 & 125 & 113 & I & 13 \\
\hline 106 & 113 & 125 & 126 & 110 & I & 13 \\
\hline 105 & 118 & 126 & 127 & IIS & I & 13 \\
\hline 106 & inc & 127 & $12 n$ & ite & , & 13 \\
\hline 107 & IIK & 128 & 129 & 117 & I & 13 \\
\hline 138 & 117 & 120 & 130 & $11 P$ & , & 13 \\
\hline 109 & 118 & isc & 131 & 113 & 1 & 13 \\
\hline 110 & 110 & 11 & 132 & 120 & I & 13 \\
\hline 11 & 121 & 133 & 130 & 122 & ; & 13 \\
\hline 112 & 122 & 130 & 135 & 123 & I & 13 \\
\hline 13 & 123 & ije & 136 & 120 & 1 & 13 \\
\hline$\cdots$ & 120 & i3e & 137 & 125 & i & 13 \\
\hline i1s & 125 & 137 & $13:$ & $: 26$ & I & 13 \\
\hline 116 & 126 & 139 & 199 & 127 & ; & 13 \\
\hline$\therefore 7$ & 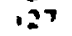 & 139 & 1ecic & 120 & I & 13 \\
\hline 110 & 129 & 100 & 101 & 129 & , & 13 \\
\hline 119 & 129 & 101 & 102 & 130 & I & 13 \\
\hline 120 & 130 & 102 & 103 & 131 & ; & 13 \\
\hline 121 & 131 & 183 & 108: & 132 & i & $? 3$ \\
\hline 122 & 133 & 105 & 106 & 130 & 8 & 13 \\
\hline 123 & 130 & 106 & 107 & 135 & i & 13 \\
\hline 120 & 135 & 187 & 186 & 130 & ; & 13 \\
\hline 125 & 136 & 100 & 109 & 137 & I & 13 \\
\hline 126 & $1: 7$ & ing & 150 & 130 & I & 13 \\
\hline 127 & $13 i$ & $\because 0$ & $1<1$ & 139 & ' & 13 \\
\hline 120 & 139 & .51 & 152 & $1+0$ & I & 13 \\
\hline 129 & ito & $1 \times 2$ & 183 & 191 & 1 & 13 \\
\hline 130 & in1 & 153 & 1s: & 102 & 1 & 13 \\
\hline 131 & 1612 & 15e & ise & 105 & ' & 13 \\
\hline 132 & 103 & ias & 156 & ien & 1 & 13 \\
\hline 133 & $10 \mathrm{C}$ & 127 & 150 & $10 x$ & 8 & 13 \\
\hline 138 & 196 & 158 & $1<0$ & 107 & ' & \\
\hline 135 & 107 & 150 & 150 & 100 & & \\
\hline
\end{tabular}




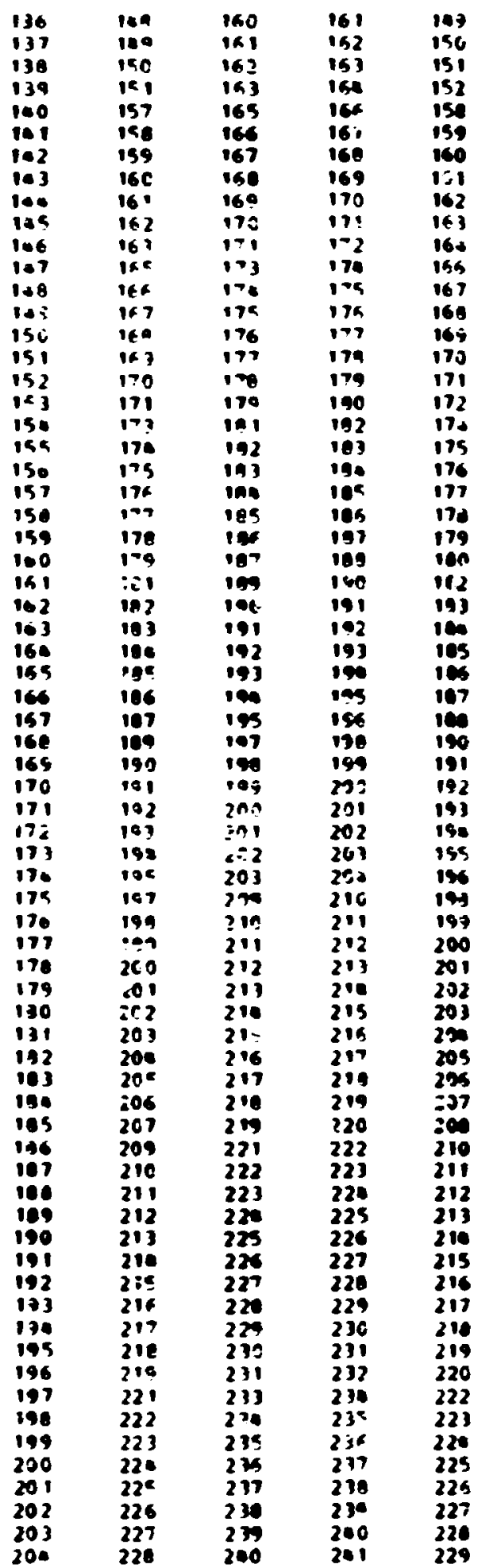




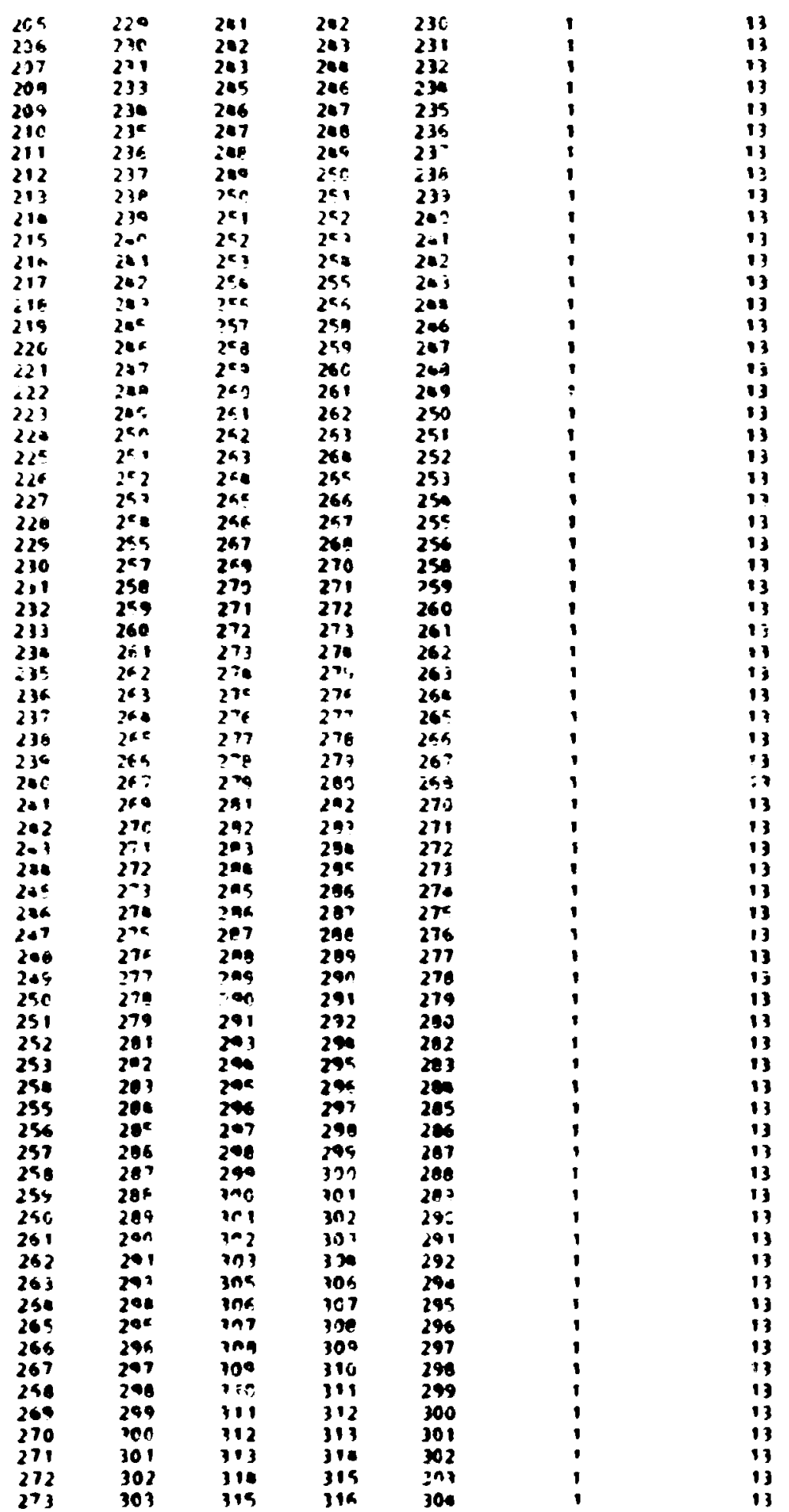




\begin{tabular}{|c|c|c|c|c|c|c|}
\hline 270 & $30<$ & 317 & 310 & 306 & 1 & 11 \\
\hline 275 & 305 & 318 & 319 & 307 & $!$ & 13 \\
\hline 277 & 3.0 & 320 & 321 & 309 & 1 & 13 \\
\hline $\begin{array}{l}278 \\
275\end{array}$ & חִ & $\begin{array}{l}121 \\
132\end{array}$ & $\begin{array}{l}322 \\
323\end{array}$ & $\begin{array}{l}310 \\
311\end{array}$ & ! & 11 \\
\hline 250 & וי & 123 & 328 & 112 & 1 & 13 \\
\hline 231 & 312 & 32 & 325 & 313 & 1 & 13 \\
\hline 292 & 313 & $32^{\circ}$ & 326 & 310 & 1 & 1 . \\
\hline 203 & $31:$ & 126 & $32^{7}$ & 315 & ' & 13 \\
\hline 254 & 316 & 327 & 329 & 316 & 1 & i3 \\
\hline 235 & 317 & $12^{\circ}$ & (3) & 316 & 1 & 13 \\
\hline 285 & 318 & COC & 331 & 319 & 1 & 13 \\
\hline 287 & 310 & 131 & 332 & 320 & 1 & 13 \\
\hline $28 \mathrm{a}$ & 320 & 332 & 133 & 321 & 1 & 13 \\
\hline 207 & 321 & 133 & 330 & 322 & 1 & 13 \\
\hline 290 & 322 & 330 & $33^{2}$ & 323 & 1 & נו \\
\hline 291 & 323 & 335 & ;36 & $32 a$ & 1 & 13 \\
\hline 232 & 320 & 336 & 377 & 329 & 1 & 13 \\
\hline 253 & 125 & 377 & 339 & 326 & ' & 11 \\
\hline 290 & $32^{*}$ & 30 & 339 & $3<?$ & 1 & ; \\
\hline 295 & 327 & 339 & 300 & 320 & 1 & 18 \\
\hline 296 & 329 & 301 & 302 & 330 & 1 & 13 \\
\hline 297 & 330 & 302 & 303 & 331 & 1 & 13 \\
\hline 298 & 331 & 303 & 300 & 332 & 1 & 13 \\
\hline 299 & 332 & 394 & 305 & 333 & 1 & 13 \\
\hline wo & 333 & 305 & 306 & 330 & 1 & 13 \\
\hline 301 & 330 & $300^{\circ}$ & $30 ?$ & 335 & 1 & 13 \\
\hline 302 & 335 & 347 & 309 & 336 & 1 & 13 \\
\hline 303 & 336 & 30.9 & 300 & 337 & 1 & 11 \\
\hline 3ne & 377 & 349 & j<c & 338 & 1 & 13 \\
\hline 305 & 738 & 350 & 351 & 337 & 1 & 13 \\
\hline $30 t$ & $3 ? 9$ & $3 \times 1$ & 342 & 300 & 1 & 13 \\
\hline 36.7 & $x=1$ & $3<3$ & 353 & 302 & 1 & 13 \\
\hline 303 & 302 & $3^{\infty}$ & $35^{\circ}$ & 343 & 1 & 13 \\
\hline 309 & 303 & 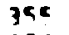 & 356 & $3: 2$ & 1 & 13 \\
\hline 312 & 300 & jea & $3 \leq 7$ & 348 & 1 & 13 \\
\hline 311 & 305 & $:: 7$ & 3<9 & 306 & 1 & 13 \\
\hline 312 & $3 \pm x$ & 150 & 359 & 30. & ' & 13 \\
\hline 313 & $30-7$ & 159 & 360 & 30? & 1 & 13 \\
\hline 314 & 308 & $3 \times 0$ & 361 & 309 & 1 & 13 \\
\hline 315 & 369 & 361 & 352 & 350 & 1 & 13 \\
\hline 315 & $3<0$ & $3 \times 2$ & $3 \times 3$ & 351 & 1 & 13 \\
\hline 317 & 341 & 353 & 360 & 352 & 1 & 13 \\
\hline 318 & jea & 365 & jak & 396 & 1 & 13 \\
\hline 319 & $3<0$ & Jae & 367 & 355 & i & 13 \\
\hline 320 & $3 \leq 2$ & $3 \times 7$ & 368 & 154 & 1 & 13 \\
\hline 321 & $35 x$ & 360: & $36^{\circ}$ & 357 & 1 & 13 \\
\hline 322 & 357 & 349 & 370 & 358 & 1 & 13 \\
\hline 323 & 358 & 370 & 371 & 359 & 1 & 13 \\
\hline $320^{\circ}$ & 359 & 371 & 372 & 360 & 1 & 13 \\
\hline 325 & 360 & 272 & 373 & 361 & 1 & 13 \\
\hline 326 & 36 1 & 373 & 370 & 362 & 1 & 13 \\
\hline 327 & 362 & 374 & $3>5$ & 363 & I & ij \\
\hline 328 & 367 & 378 & 375 & 360 & 1 & 13 \\
\hline 329 & $36 !$ & 377 & 373 & 360 & 1 & 11 \\
\hline 330 & $36^{6}$ & 378 & 379 & 367 & 1 & 11 \\
\hline 331 & 367 & 396 & 393 & $36 a$ & 1 & 13 \\
\hline 332 & 360 & 300 & 381 & $36 ?$ & 1 & 13 \\
\hline 333 & 369 & $3 A 1$ & 392 & 370 & 1 & 13 \\
\hline $33=$ & $37 c$ & 3A2 & 383 & ו737 & 1 & 13 \\
\hline 335 & ור ו & it 3 & 396 & 322 & 1 & 13 \\
\hline 336 & 372 & 3AE & 385 & 373 & $i$ & 13 \\
\hline 337 & דר & 305 & 386 & 370 & 1 & 13 \\
\hline 33A & & 3AG & 387 & 375 & 1 & 13 \\
\hline 339 & 375 & $3 R 7$ & 398 & 376 & 1 & is \\
\hline 300 & 377 & 389 & 390 & 378 & 1 & 1) \\
\hline 341 & 378 & 390 & 391 & 379 & 1 & 13 \\
\hline 302 & 379 & 391 & 392 & 380 & 1 & 13 \\
\hline
\end{tabular}




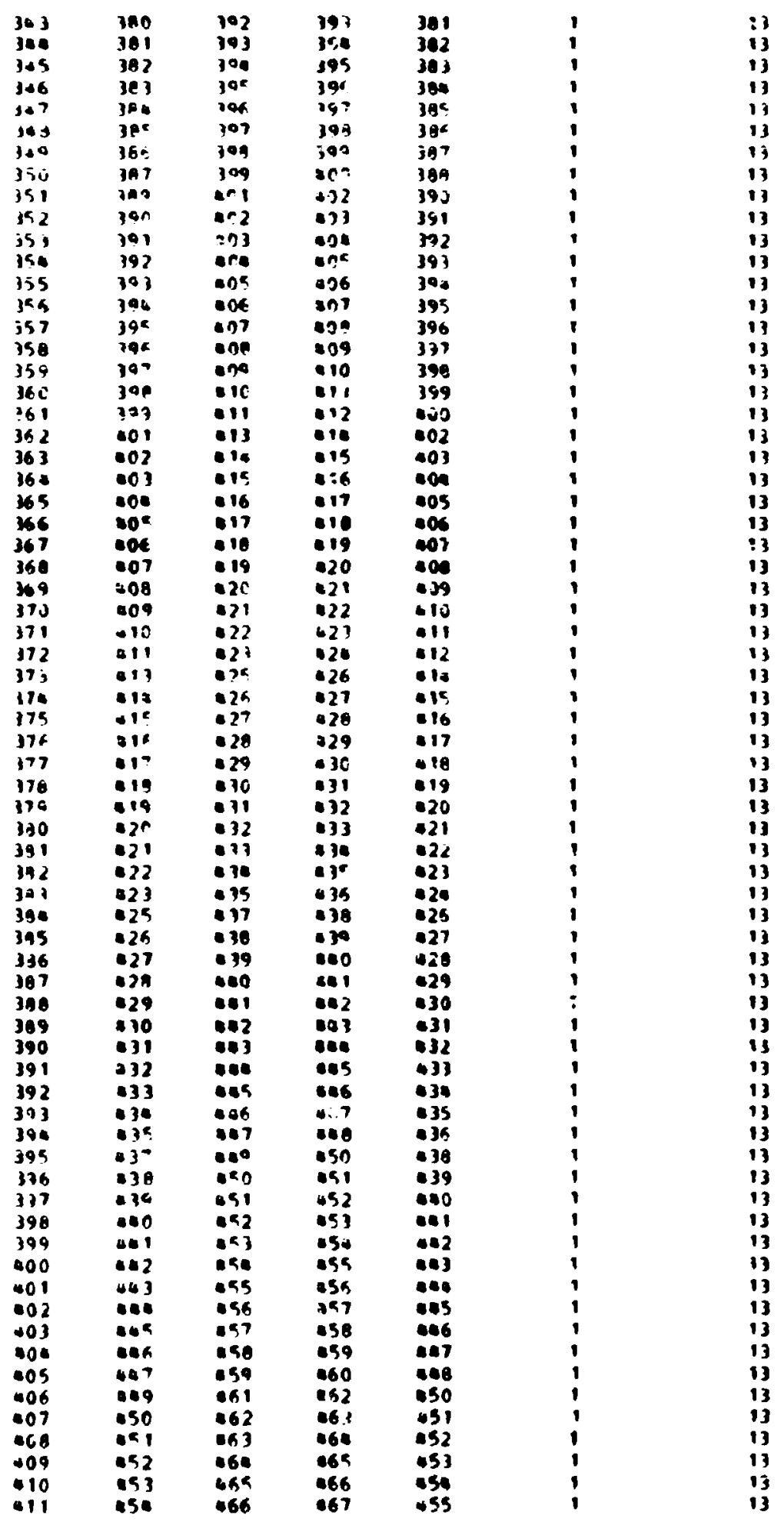




\begin{tabular}{|c|c|c|c|c|c|c|}
\hline+12 & 455 & 367 & $7 ; 6$ & 450 & 1 & $1 ?$ \\
\hline+13 & iet & 268 & 669 & $\$ 57$ & $i$ & 13 \\
\hline 314 & $0<7$ & $3 \times 3$ & $17 n$ & $\therefore 50$ & 1 & 13 \\
\hline+15 & $\therefore 58$ & 479 & +71 & 459 & 1 & :3 \\
\hline+16 & ars. & $=71$ & 372 & सरीक & 1 & 13 \\
\hline+17 & 361 & 479 & 678 & $\$ 6 \bar{Z}$ & 1 & 13 \\
\hline+18 & I6? & 474 & 475 & 467 & 1 & 13 \\
\hline+15 & uf: & 475 & $\$ 76$ & $+6+\infty$ & 1 & 13 \\
\hline$\$ 20$ & 460 & $27 \epsilon$ & $\$ 77$ & $45=$ & I & 13 \\
\hline-21 & $4 \times 5$ & 477 & 478 & 466 & 1 & 13 \\
\hline+22 & 456 & $\triangle 78$ & $\$ 79$ & 467 & 1 & 13 \\
\hline+23 & 467 & 479 & 49ก & 468 & 1 & 13 \\
\hline-24 & 366 & 490 & 4a1 & 469 & 1 & 13 \\
\hline 425 & 1164 & ar? & 382 & 670 & 1 & 13 \\
\hline 426 & 470 & $\triangle A 2$ & 483 & 471 & 1 & 13 \\
\hline 427 & 471 & 493 & 480 & 472 & 1 & 13 \\
\hline+20 & 473 & QAE & 486 & 474 & 1 & 13 \\
\hline 427 & 470 & $\because 96$ & 061 & 475 & 1 & 13 \\
\hline 430 & 675 & 487 & 488 & $27 h$ & $i$ & 13 \\
\hline 431 & 476 & 48 & 089 & 477 & 1 & 13 \\
\hline 352 & $\$ 77$ & 99 & 490 & 473 & 1 & 13 \\
\hline 433 & 478 & 490 & 491 & 479 & 1 & 13 \\
\hline 434 & 479 & 491 & 092 & 480 & 1 & 13 \\
\hline 435 & 480 & 402 & 493 & 4d 1 & 1 & 13 \\
\hline 436 & 481 & 493 & 494 & 482 & 1 & 13 \\
\hline 437 & 492 & 474 & $\$ 25$ & 483 & 1 & 13 \\
\hline 438 & 493 & $\$ 9 c$ & af & 004 & 1 & 12 \\
\hline+39 & 485 & 497 & a99 & -66 & 1 & 13 \\
\hline 140 & $48 f$ & 29ด & 099 & 487 & 1 & 13 \\
\hline $4+1$ & \&E? & 430 & sno & 439 & ; & 13 \\
\hline$+2+2$ & 488 & 500 & 501 & 489 & $i$ & 13 \\
\hline-43 & IAC & $5 ! 1$ & 502 & 490 & 1 & 13 \\
\hline $4+0$ & $49 n$ & 502 & 503 & 491 & 1 & 13 \\
\hline+45 & $4 n 1$ & 503 & 504 & 492 & 1 & 17 \\
\hline+46 & 492 & 504 & 505 & 493 & 1 & 13 \\
\hline $4+7$ & 493 & 505 & 506 & 494 & 1 & 13 \\
\hline $74 d$ & 494 & 506 & 507 & 495 & 1 & 13 \\
\hline$\triangle 4$ & $\$ 95$ & 507 & 508 & 496 & 1 & 13 \\
\hline 450 & $40 ?$ & 509 & 510 & 498 & 1 & 13 \\
\hline 451 & 498 & 510 & 511 & 499 & $i$ & $1 x$ \\
\hline 452 & 499 & 511 & 512 & 500 & 1 & 1 \\
\hline 453 & 500 & 512 & $5: 3$ & 501 & 1 & 13 \\
\hline 454 & 501 & 513 & 514 & 502 & 1 & 13 \\
\hline 455 & 502 & 514 & 515 & 503 & 1 & 13 \\
\hline 456 & 503 & 515 & 515 & 504 & 1 & 13 \\
\hline+57 & 504 & 516 & 517 & 505 & 1 & 13 \\
\hline 458 & 505 & 517 & 518 & 506 & 1 & 13 \\
\hline 459 & $=06$ & 518 & 519 & 507 & 1 & 13 \\
\hline प́́o & <97 & c 19 & 520 & 508 & 1 & 13 \\
\hline Lh 1 & 909 & 521 & 522 & 510 & 1 & 17 \\
\hline 462 & 510 & 532 & .23 & 511 & 1 & 13 \\
\hline 453 & 511 & 423 & 524 & 512 & 1 & 13 \\
\hline 464 & c 12 & 524 & 325 & 513 & 1 & 13 \\
\hline 465 & 517 & 524 & 526 & 5,14 & $i$ & 13 \\
\hline 466 & 510 & $52 k$ & 527 & 515 & 1 & 13 \\
\hline 407 & 515 & 527 & 528 & 516 & 1 & 13 \\
\hline 459 & j16 & $<28$ & 529 & 517 & 1 & 13 \\
\hline 4n 9 & 515 & $=24$ & 530 & 510 & $i$ & 15 \\
\hline 470 & $51 \mathrm{~A}$ & 530 & 531 & 519 & 1 & 13 \\
\hline 471 & 510 & 531 & \{3\} & 520 & 1 & 13 \\
\hline 472 & 521 & 533 & 530 & 522 & 1 & 13 \\
\hline 473 & 522 & C 34 & 535 & 523 & 1 & $1 ?$ \\
\hline 474 & $42\}$ & 534 & 536 & 524 & 1 & 13 \\
\hline 415 & 52. & 536 & 537 & 525 & 1 & 13 \\
\hline 476 & 525 & 637 & 538 & 526 & $i$ & 13 \\
\hline 477 & 526 & 539 & 539 & 527 & $i$ & 13 \\
\hline 478 & 527 & $<39$ & $r, 40$ & 528 & $i$ & 13 \\
\hline 479 & 528 & 540 & 541 & 529 & 1 & 13 \\
\hline 490 & 529 &, 41 & 542 & 530 & 1 & 13 \\
\hline
\end{tabular}


ici

\begin{tabular}{|c|c|c|c|c|c|c|}
\hline 135 & 530 & 507 & $\cos$ & 5.1 & 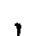 & 13 \\
\hline$d y_{2}$ & 531 & $<47$ & 584 & 5,32 & ; & 13 \\
\hline [A \} ? & 5 & SES & $\therefore x$ & 53 & 1 & $i$ \\
\hline $49 i$ & 570 & caf & $5=7$ & $=35$ & ; & 13 \\
\hline da & $53^{\circ}$ & $=27$ & द & 53 & i & 13 \\
\hline$\rightarrow 9 E$ & $5 \geq 6$ & $5+6$ & Sa? & 537 & ; & 13 \\
\hline $\begin{array}{r}5 z \\
+B 1\end{array}$ & דו & $c 9$ & 550 & 538 & ; & 3 \\
\hline tag & 538 & 590 & 551 & 539 & ; & 13 \\
\hline $\begin{array}{r}409 \\
89\end{array}$ & $<39$ & 551 & $\varangle<2$ & 500 & 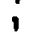 & 13 \\
\hline $\begin{array}{r}+30 \\
\end{array}$ & 500 & $<52$ & 553 & 541 & ; & 13 \\
\hline 91 & 501 & $5<3$ & ase & 542 & ; & 13 \\
\hline+92 & 502 & $550^{\circ}$ & sace & 543 & ; & 13 \\
\hline 493 & 54 & sed & 556 & 540 & ? & 13 \\
\hline 998 & sea & 557 & 558 & 546 & ; & 13 \\
\hline 375 & Sen & ६58 & 559 & 547 & $i$ & 13 \\
\hline .96 & 547 & 559 & 560 & 548 & ; & 13 \\
\hline 197 & 548 & $\$ 60$ & 561 & 540 & i & 3 \\
\hline 698 & & 561 & 562 & 550 & i & 13 \\
\hline og & $\Leftrightarrow<0$ & 562 & 5.53 & 551 & ; & 13 \\
\hline 500 & 51 & 563 & 560 & 552 & ; & 13 \\
\hline 501 & $\leq 2$ & $<\times a$ & 565 & 553 & ; & 13 \\
\hline 502 & 553 & $5 \times 5$ & 566 & 554 & 1 & 13 \\
\hline 501 & उद & CKA & $=67$ & 555 & 1 & 13 \\
\hline 50.4 & & & & 556 & $i$ & 13 \\
\hline 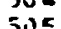 & $=2$ & $=6$ & $\leqslant$ & $<<$ & ; & 13 \\
\hline 506 & ऽৎя & 570 & 571 & 559 & i & 13 \\
\hline 56.7 & 59 & $\Leftrightarrow 1$ & ¿72 & 560 & i & 13 \\
\hline 500 & 560 & $\therefore 72$ & 573 & 561 & ; & 13 \\
\hline 509 & $r, 61$ & 573 & 578 & 562 & i & 13 \\
\hline 510 & 562 & 574 & 575 & 563 & ; & i3 \\
\hline 511 & $5 \times 3$ & 575 & 575 & 564 & ; & 13 \\
\hline 512 & $\$ 64$ & 5.76 & 577 & 565 & ; & 13 \\
\hline 5 & $5=$ & SI & 5 & 5 & , & 3 \\
\hline 514 & 566 & 578 & 575 & 567 & ; & 13 \\
\hline 51 & 567 & 579 & 48 & 568 & 1 & is \\
\hline 516 & 569 & 581 & 582 & 570 & ; & $?$ \\
\hline 59 & 570 & 302 & 3 & 571 & i & iz \\
\hline 518 & 571 & 503 & 580 & 572 & i & 13 \\
\hline 519 & 572 & 580 & 585 & 5 & i & 13 \\
\hline 520 & 573 & 585 & 586 & 57 & i & 13 \\
\hline 521 & 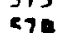 & 586 & 581 & 575 & ; & 3 \\
\hline 522 & 575 & 587 & 5 SBE & 576 & i & 13 \\
\hline & 5 & 589 & 590 & 582 & $i$ & 9 \\
\hline & 5 & 590 & 59 & 58 & $;$ & 9 \\
\hline 25 & 583 & 5 & 59 & 58 & ; & 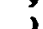 \\
\hline 526 & 580 & 502 & 593 & 585 & ; & $q$ \\
\hline & $5 \theta^{2}$ & $59 ?$ & 500 & 586 & 1 & 9 \\
\hline 528 & 589 & 504 & 595 & 587 & ; & 9 \\
\hline
\end{tabular}




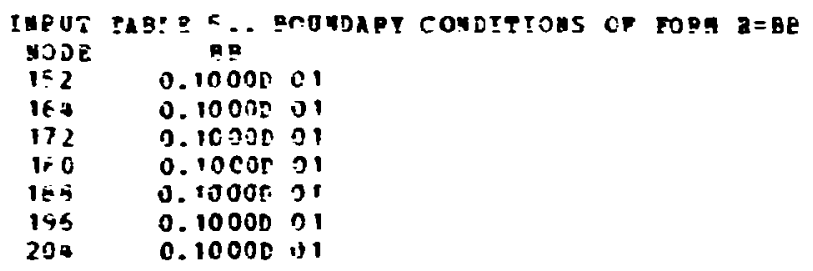

I WP OT TLBL 7 .. SEEPAG

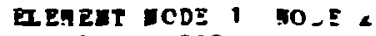

135 $268 \quad 220$

$196-22 \mathrm{C}$

$207 \quad 232$

$218 \quad 245 \quad 2 \div 6$

229 25A 269

$240 \quad 268 \quad 290$

$251 \quad 29 \mathrm{C} \quad 202$

$252 \quad 292$ 30?

$273 \quad 304 \quad 316$

$283316 \quad 328$

$295 \quad 325 \quad 340$

$306 \quad 340 \quad 352$

$317 \quad 352 \quad 360$

$328 \quad 364 \quad 376$

$339 \quad 376 \quad 386$

$350 \quad 368$ ano

$361 \quad 400 \quad \$ 12$

$\begin{array}{ll}372 & 412 \quad 426\end{array}$

$\begin{array}{lll}383 & 424 & 36\end{array}$

$405 \quad 408 \quad 50$

$116 \quad 460 \quad 47$

$427 \quad 872 \quad 484$

$438 \quad 484 \quad 496$

$449 \quad 496 \quad 508$

$460 \quad 509 \quad 520$

$471 \quad 520 \quad 532$

$+82 \quad 432<49$

$+93 \quad 544 \quad 596$

$50 a \quad 556 \quad 5 \% 8$

$515 \quad 569 \quad 590$

$515 \quad 579 \quad 580$

$514 \quad 578 \quad 579$

$513 \quad 577 \quad 578$

$512576 \quad 577$

$522575 \quad 598$

$522 \quad 587 \quad 589$ 
응

00000000000000000080000000000000000000

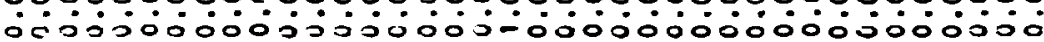

$0000003000030010000=000000000000000300$

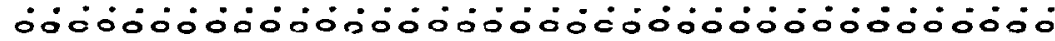
00000000000000000000002000000000000000

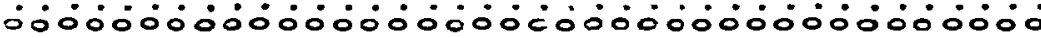
00000000000000000000000000000030000000

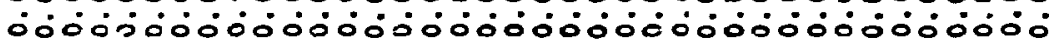

!

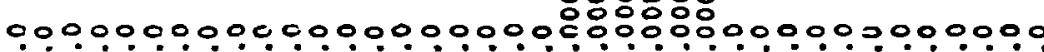

응웅ㅇㅇㅇㅇㅇ

응영용영영웅

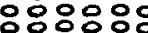

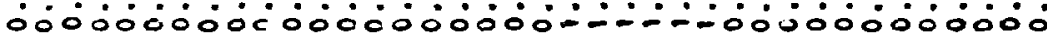

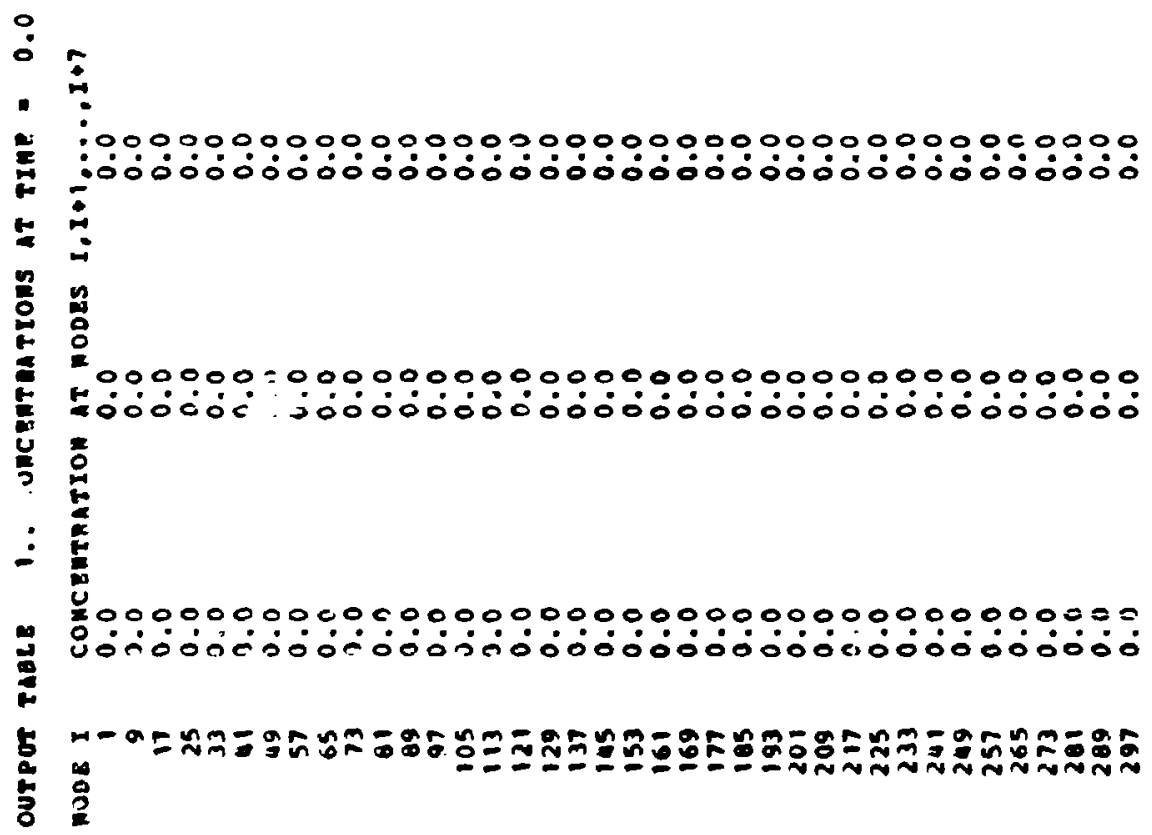


000000000000000000000090000000000000

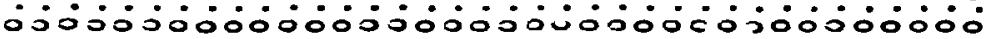

000000000000000000000000000000000000 000000000000

$00000000000000=000000000000000000000$

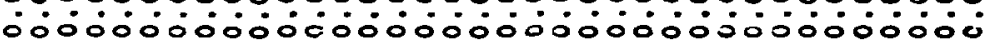

000000000000000000000000000000000000

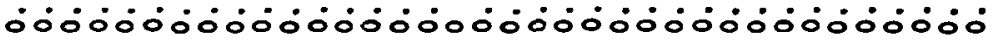

000000000000000000000000000000000000

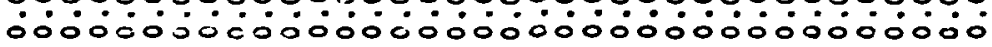

0000005000000000000000000090000000000

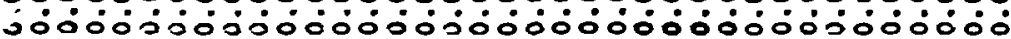

0000000000000000000000000000000000000

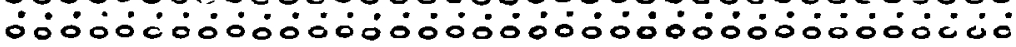

$00000000<0000000000000000000000000000$

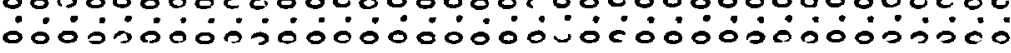

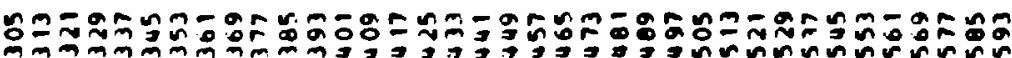




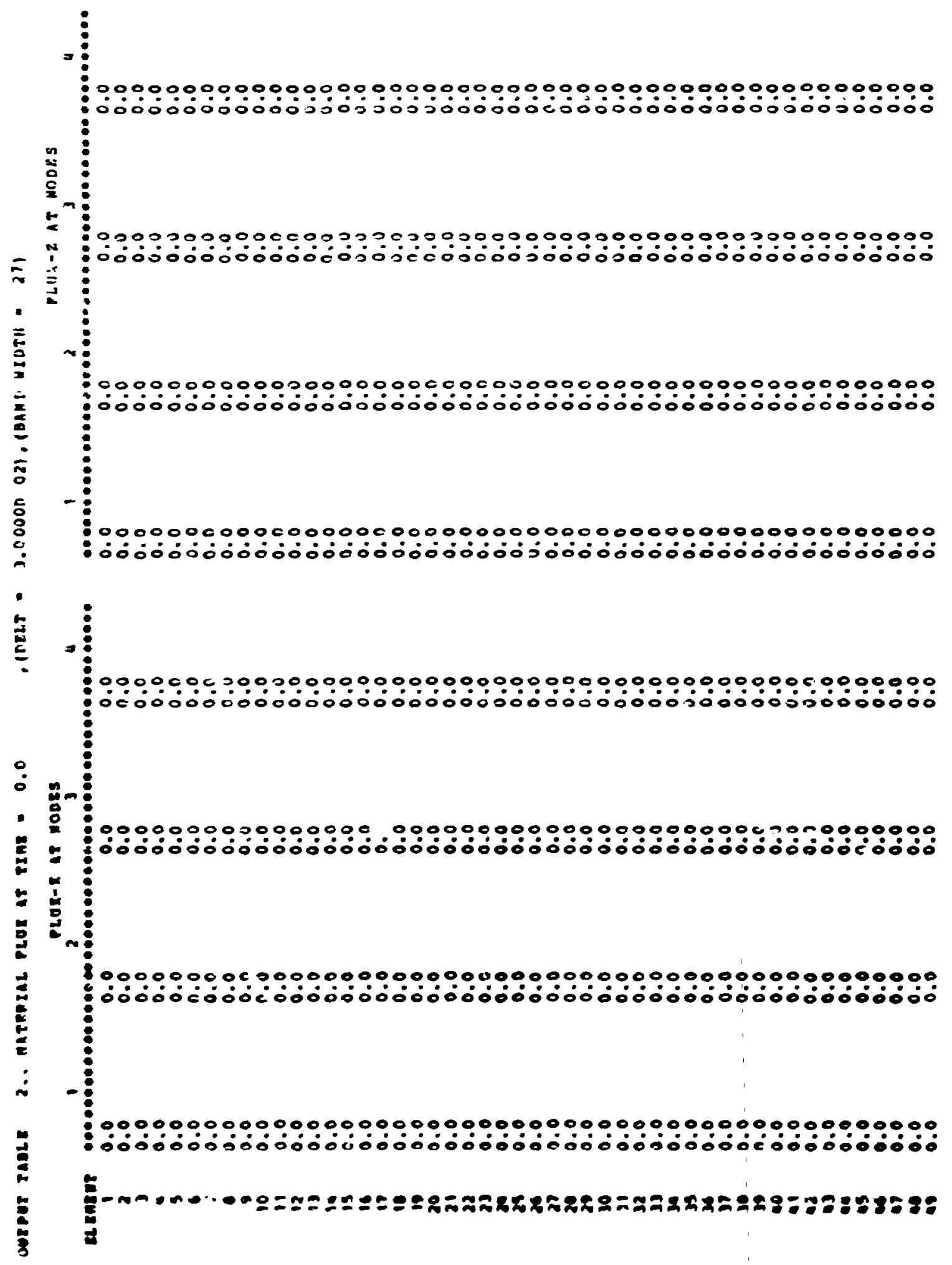


00000900000000000000000000000000000000000000000000

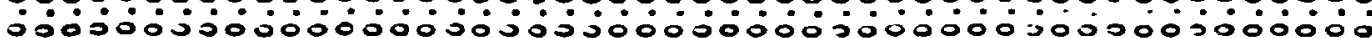

00009009309090900000000000900939009093009090000000 ○0000000000000000000000000000000000000000

00000000000000000000000000000000000000000000000000

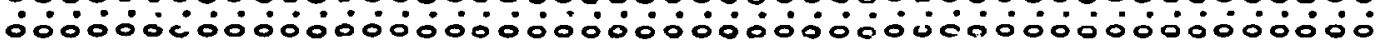

$00000000000000000000000000 r_{1}-1000000000000000000000$

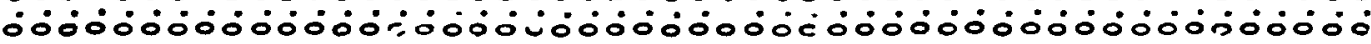

00000001000000000000000000000000000000000000000000

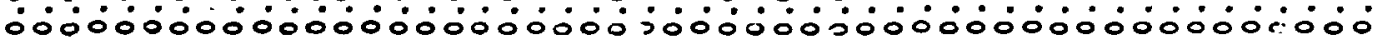

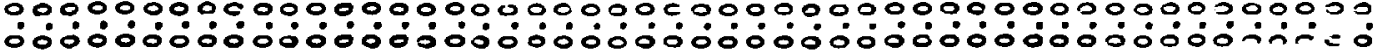

0000000000000000000000000004000.300000000000000000

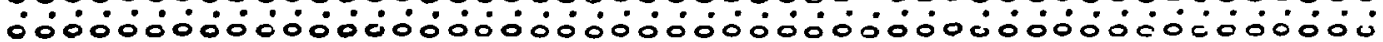

00000000000000000000000000000000000000000000000000 - $0_{00000000000000}$

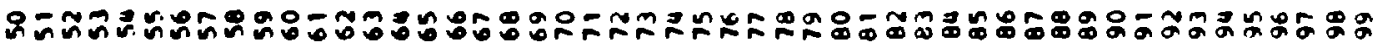


c 00000000000000000000000000000000000000000000 荡000 1.0000000000000000000000000000000000000010000009000

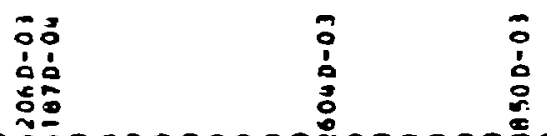

$0030000300000000000000000000500000000000000000 \$ 000$

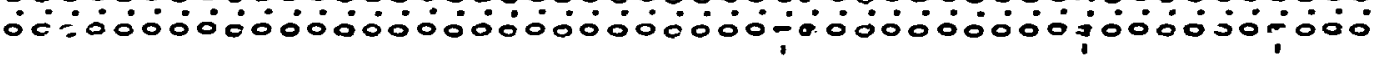

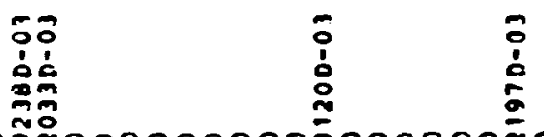

0000000000000000000000000000 20 $0000000000000000 \overline{1} 000$

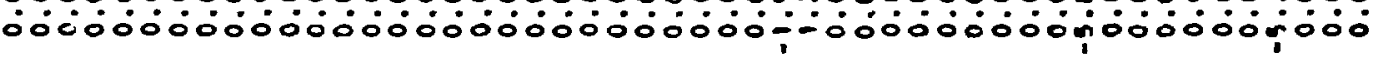

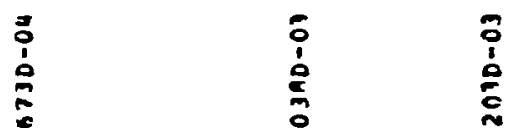

00000000000000000000000000000500000000000000000000

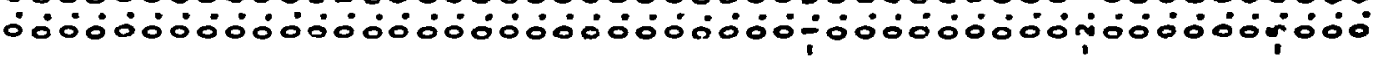

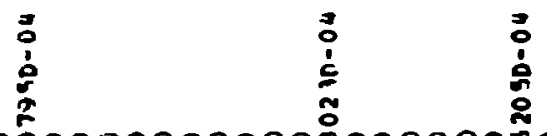

00000000000000000000000000000000000000000000000000

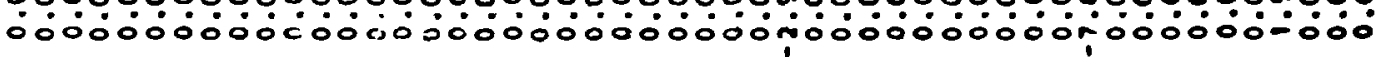

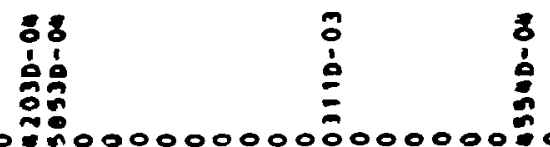
@ं00000000000000000000000000000400000000000000000000

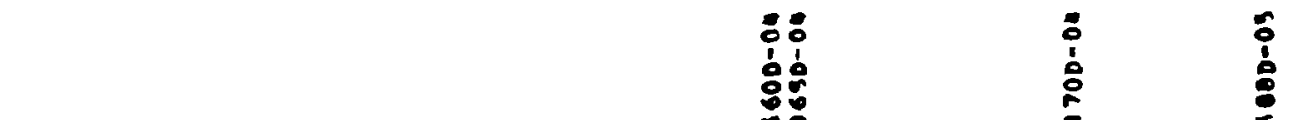
$00000000000000000000000000005000000000 \overline{0} 0000001000$

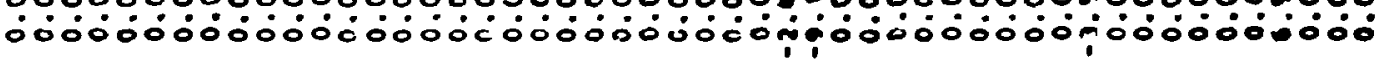

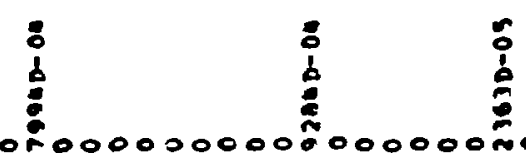
00000000000000000000000000000700000000000000001000

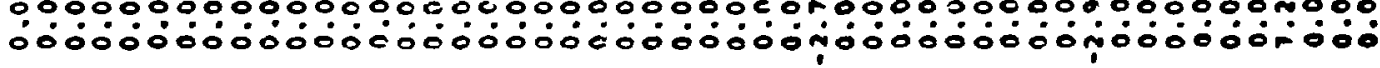

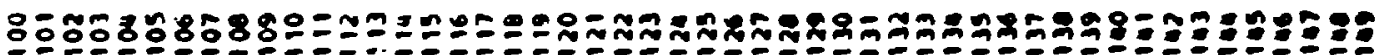




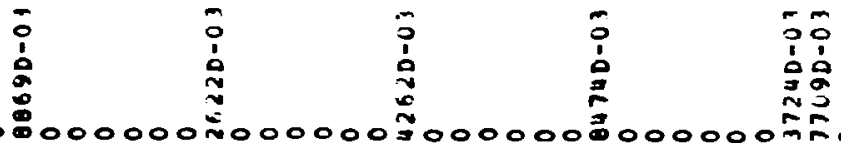

00000000000000000300000000000007700000000000000000

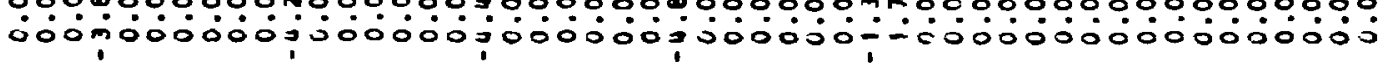

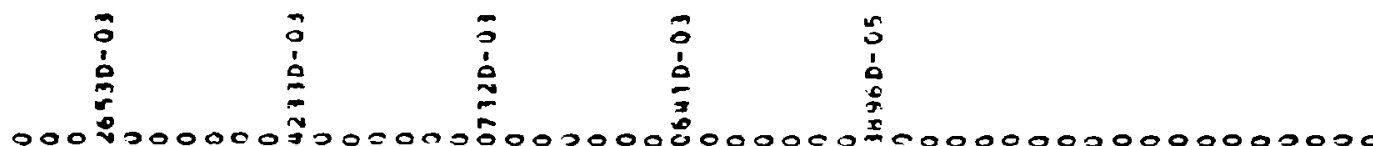
000

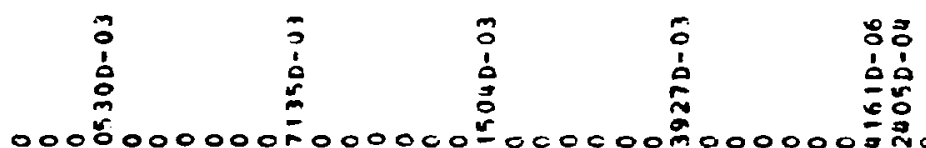

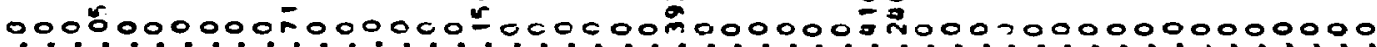

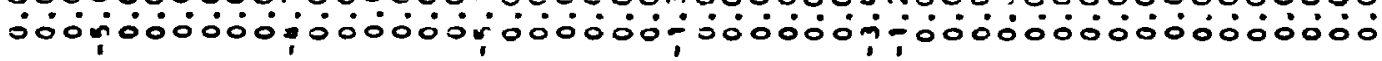

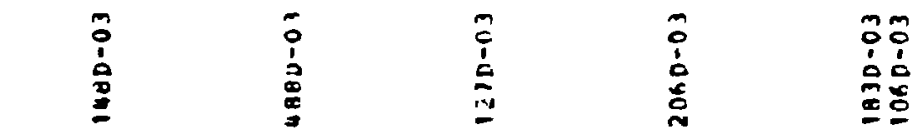

$000 \tilde{n} 000000 \bar{z} 000000 \overline{0} 000000$ N000000 000000000000000000

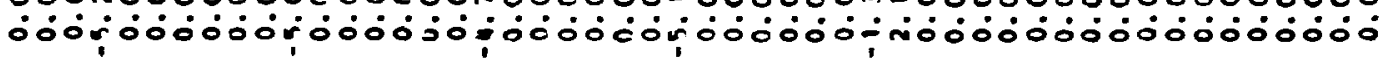

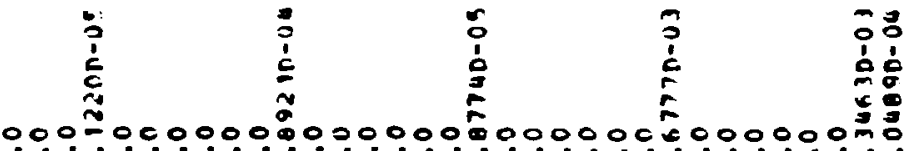
$000 \div 00000000.0000000000040000001000000000000003000$

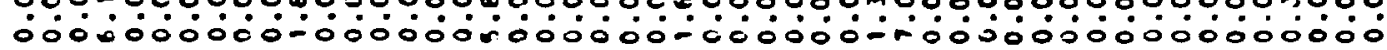

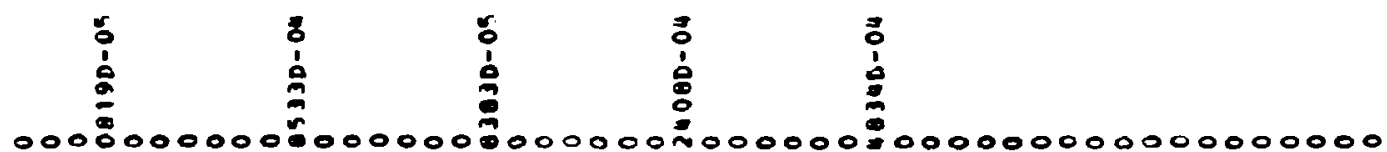

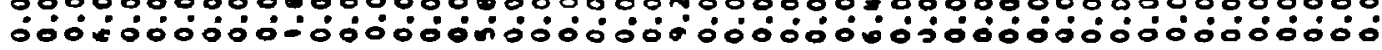

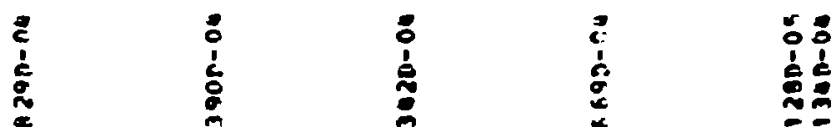
$00000000006000000000000 \pm 0000000=00000000000000000$

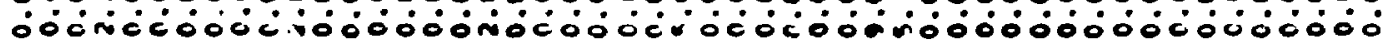

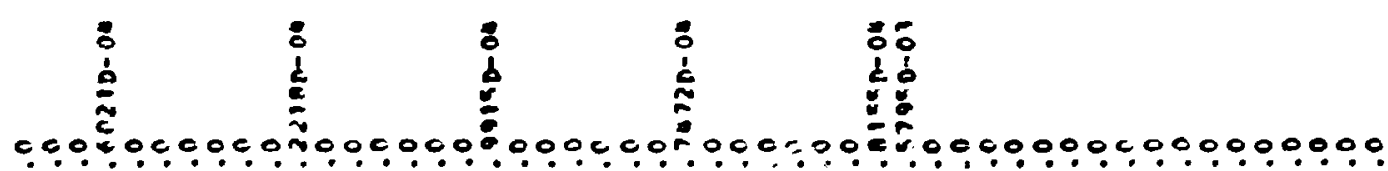

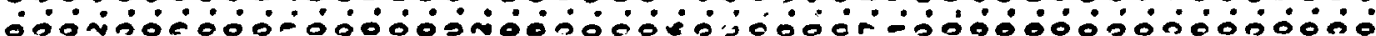


00000900000000000000000090000000000000000000000000

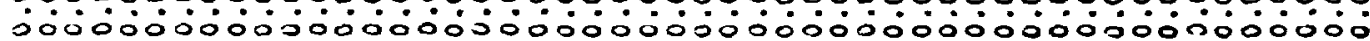

00000900000000090090009009090000000900009000000900

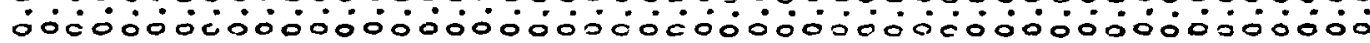

00000000000000000000000000000000000000000000000000 ठ000

$00000000000000000000000000000000=00000000000000000$

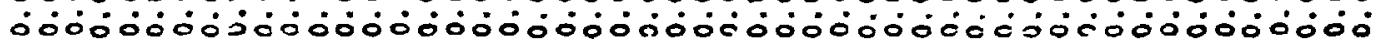

00000000000000000000000000000000000000000000000000 000000ं00

00000000000000000000000000000000000000000000000000

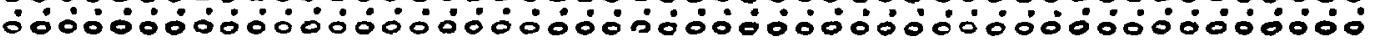

00000000000000000000000000000000000000000000000000

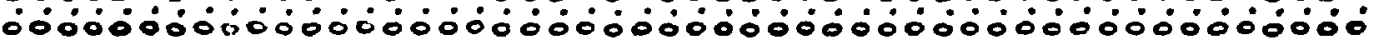

00000000000000000000000000000000000000000000000100

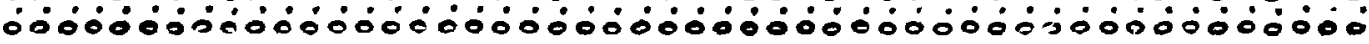

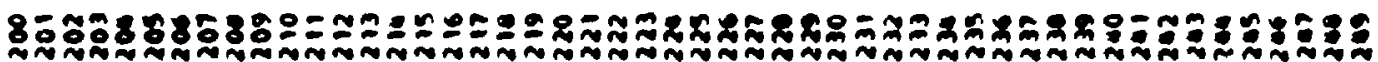


00000000000000000000000000000000000000000000000000

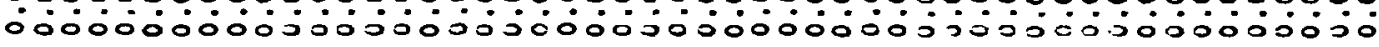

00000000000000000000000000000000000000000000000000

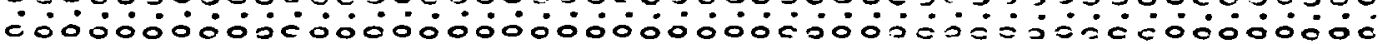

$00000000000000000000000000000000000000=00000000000$

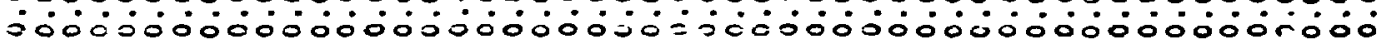

00000000000000000000000000000000000000000040000000

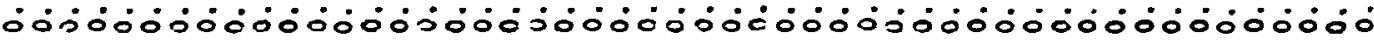

00000000000000000000000000000030000000000000000000

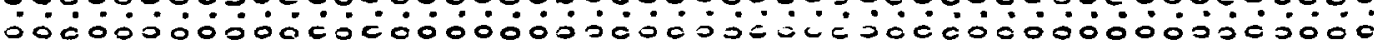

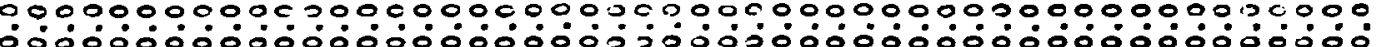

$c 0=00000000000000000000000000000000000000000000000$

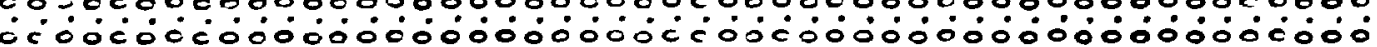

00000000000000000000000000000000000000000000000000

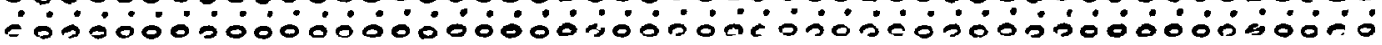

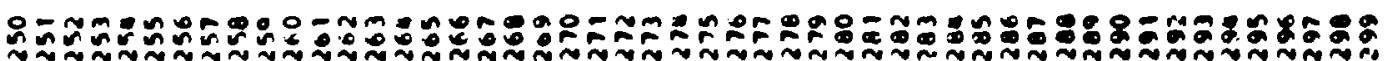


00000000000000000000000000000000000000000000000000

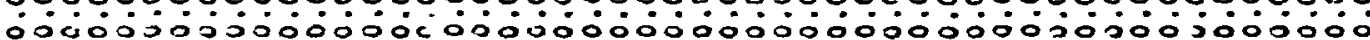

00000000000000000000000003002000000000000000000000

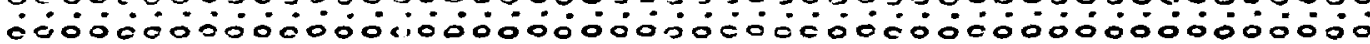

$0000000000000000000000000000<000000000000000000000$

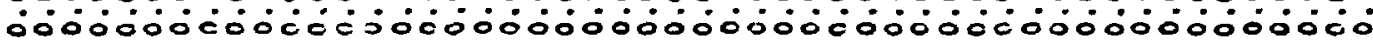

00000000000000000000000000000000000000000000000000

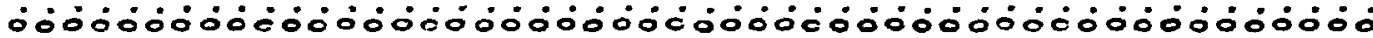

00600000000000000000000000000000000000000000000000

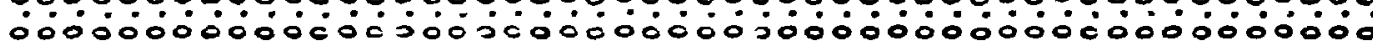

00000000000000000000000000000000000000000000200000

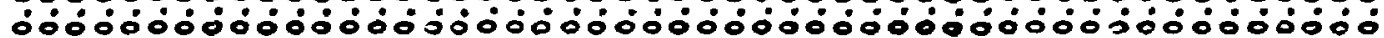

$0000000000000000<000000000000000000000000000000000$

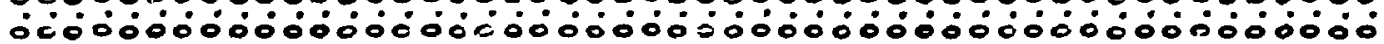

00000000000000000040000000000000000000000000000000

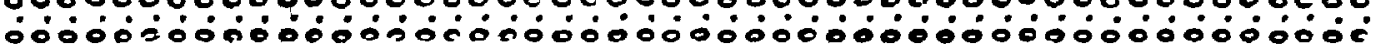

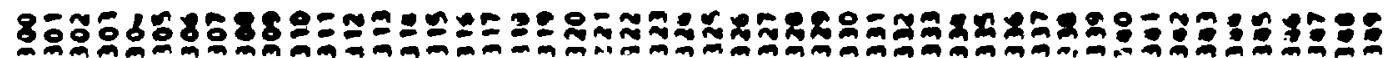


00000000000000000000000000000000000000000000000000

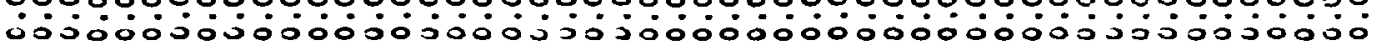

00000000000000000200000000000000000000000000000000

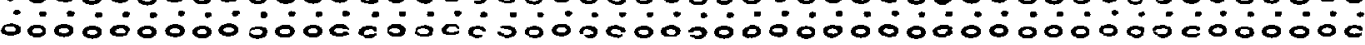

00000000000000000000000000000000000000000000000000

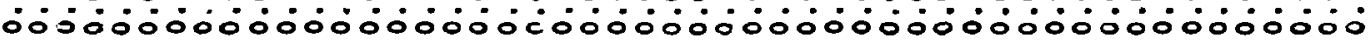

00000000000000000000000000000000000000000000000000

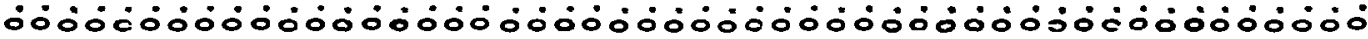

00000000000000000000000000000000000000000000000000

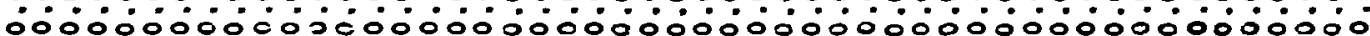

00000030000000030000000000040000000000030000000000

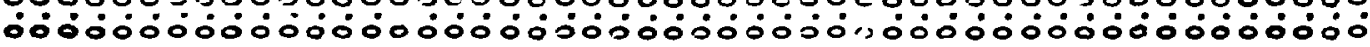

00000000000000000000000000000000000000000000000000

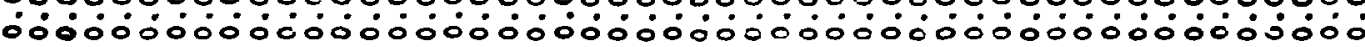

00000000000000000000000000000000000000000000000000

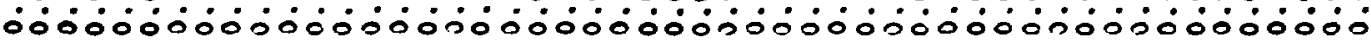

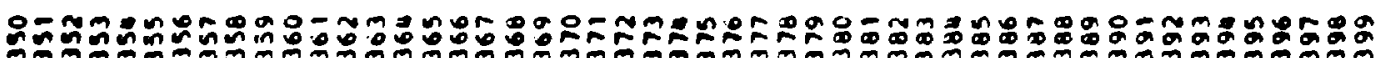


$0900000000000000000=000000000000000000030000000000$

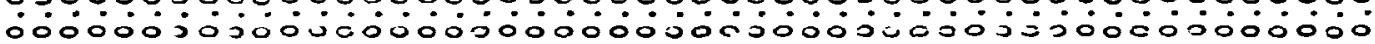

$n=000000,20100000200000000000020000000000000000000$

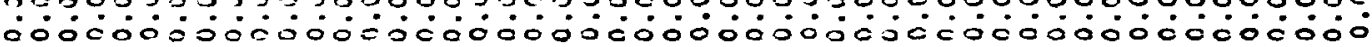

$0000000000 C c 0 c 000000000000000=00000000000000000000$

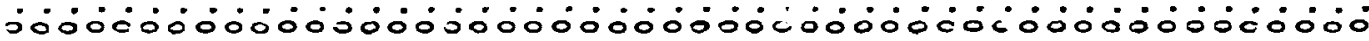

00000000000000000000000000000000000000000000000000

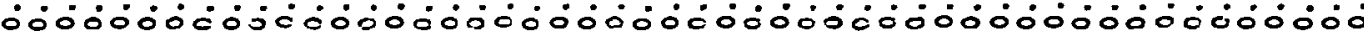

$00000000000000000000=00000000000000000000000000000$

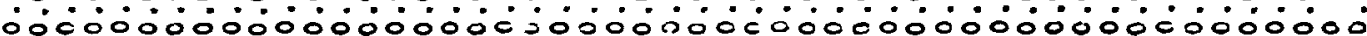

00000000000000000000030000000000000000000000000000

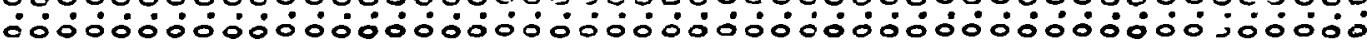

00000000000000000000000000000000000000000000000000

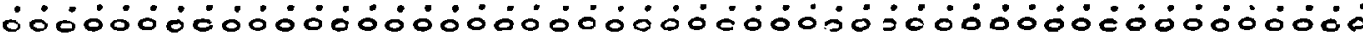

$00000000000000000000000000000000000000000000000-00$

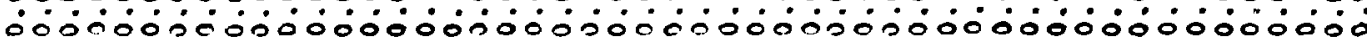




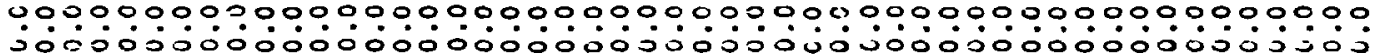

$0000000000000000000=000000000006000000000-000309000$

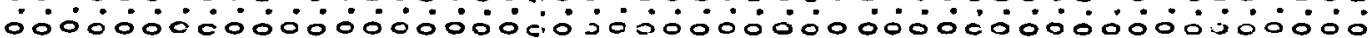

00000000000000000000000000000000000000000000000000

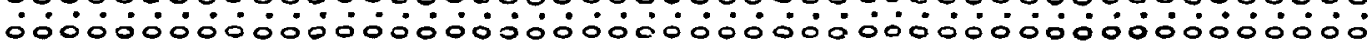

000000000000000003000000000000000000000130000000000

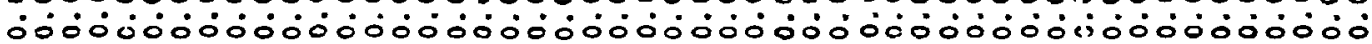

00000000000000000000000000000000000000000000000000 $000000000000 j 000000000000000000000000000000000 j 0$

00000000000000000000000000000000000000000000000000 -

$00000000000000000000000000000000000=00000000000000$

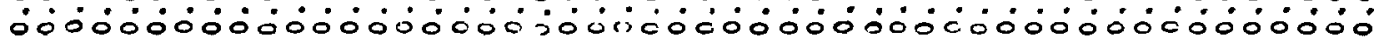

00000000000000000000000000000000000000000000000000

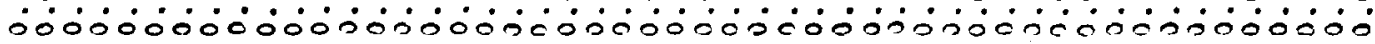

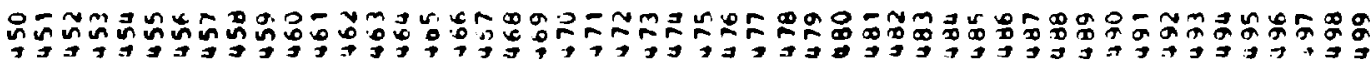


00000000009000000000000009000

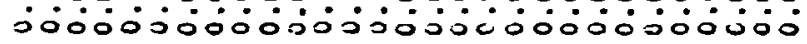

00000000000000003000000000000

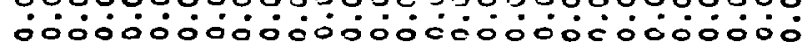

$00<0000000000000000<000000000$

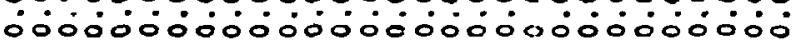

00000000000000000000000000000

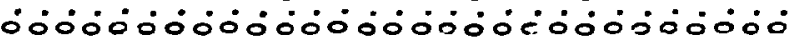

00000000000000000000000000000

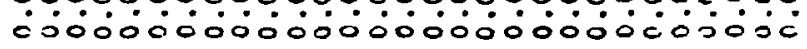

00000909000090090000000000000

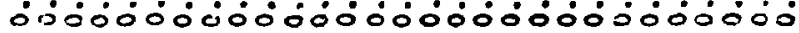

$00000000000 n=0000000000000000$

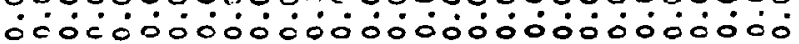

00000000000000000000000000000 j0000000000090000000000040000.

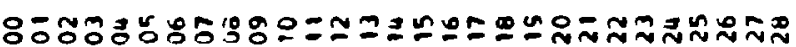
O을 
iio

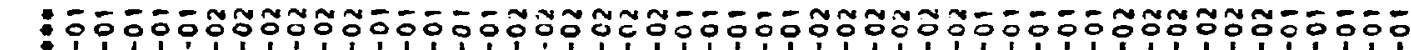

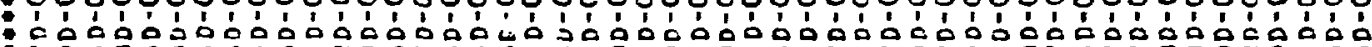

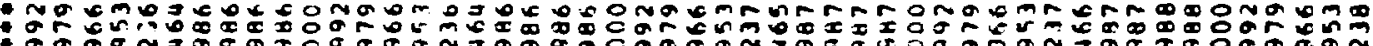

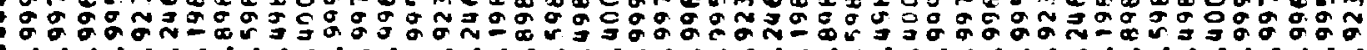

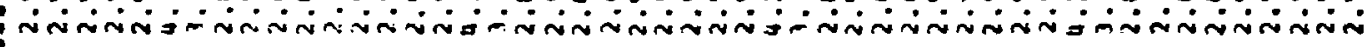

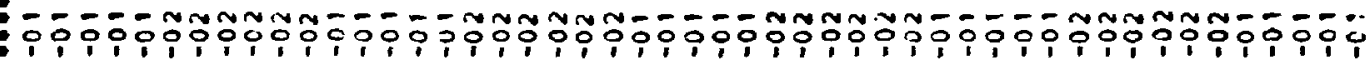

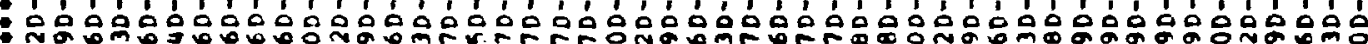

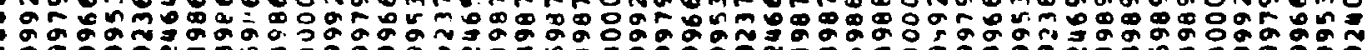

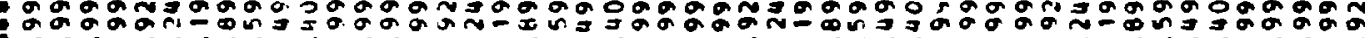

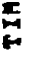

空

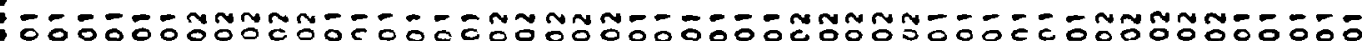

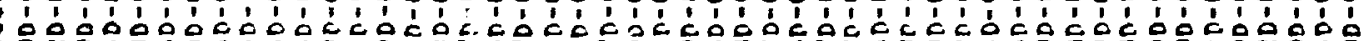

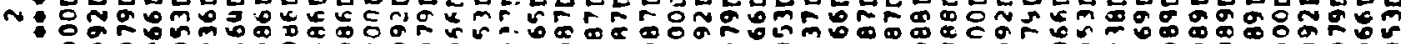

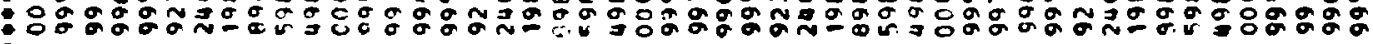

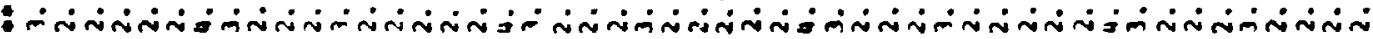

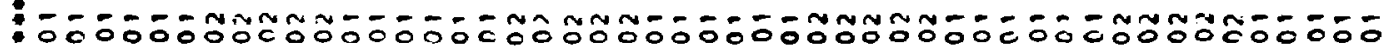

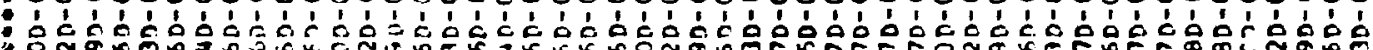

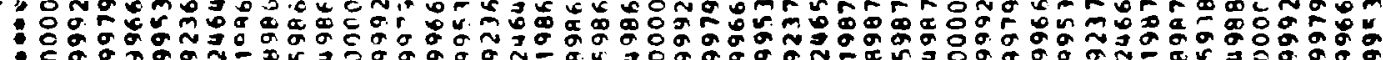

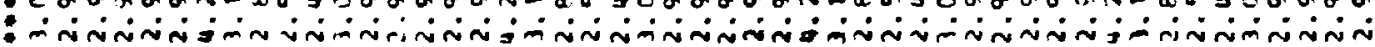

$$
\text { 点 }
$$

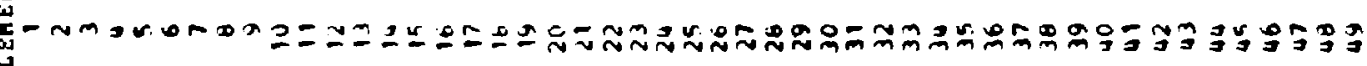




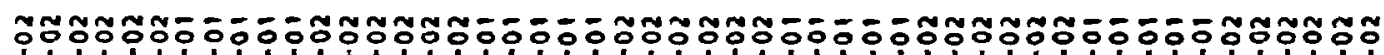

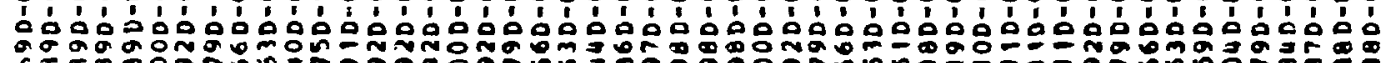

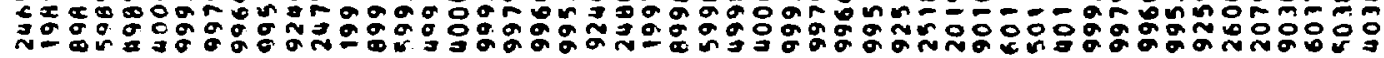

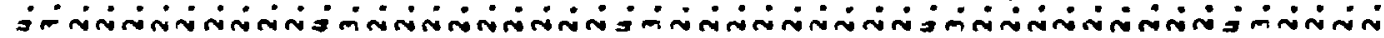

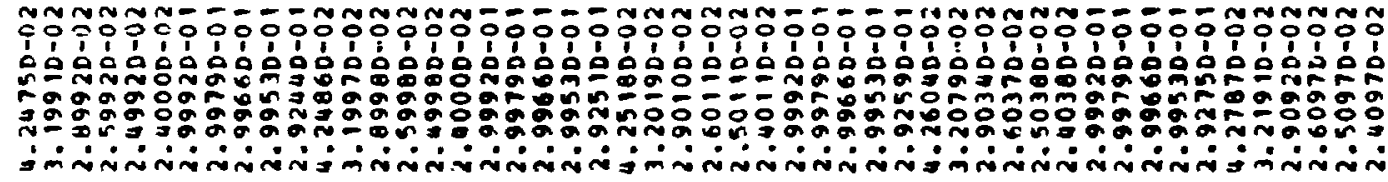

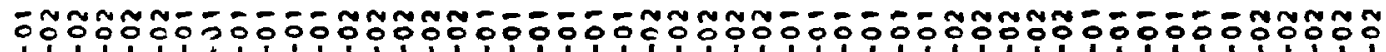

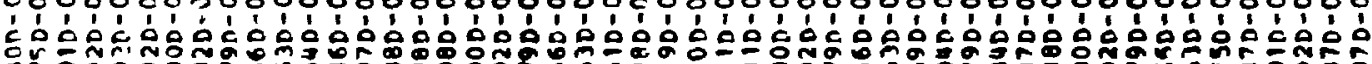

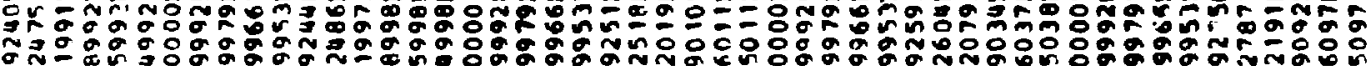

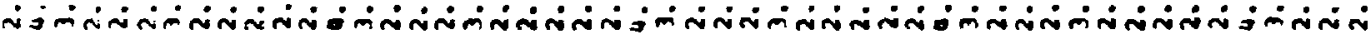

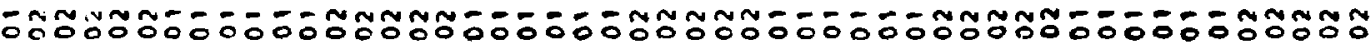

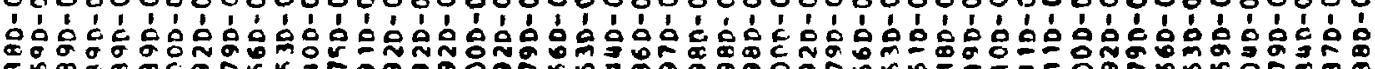

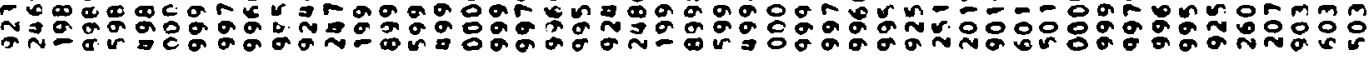

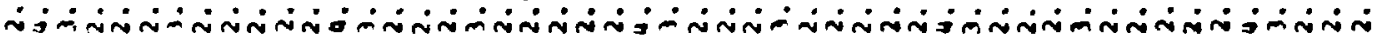




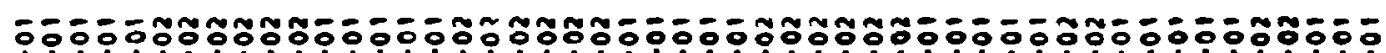

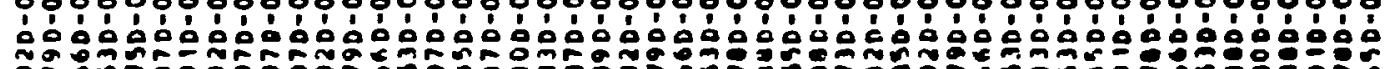

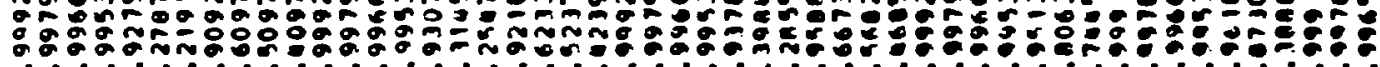

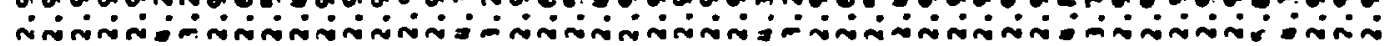

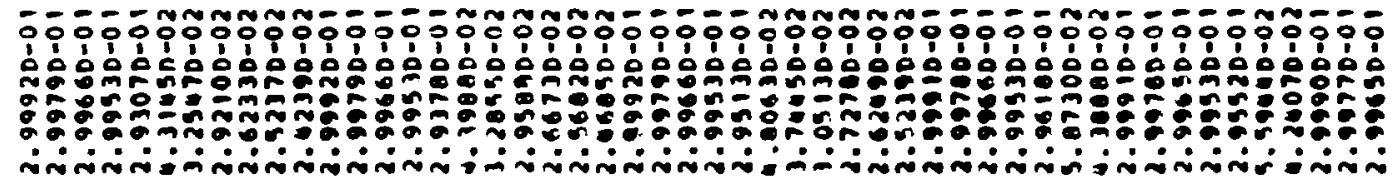

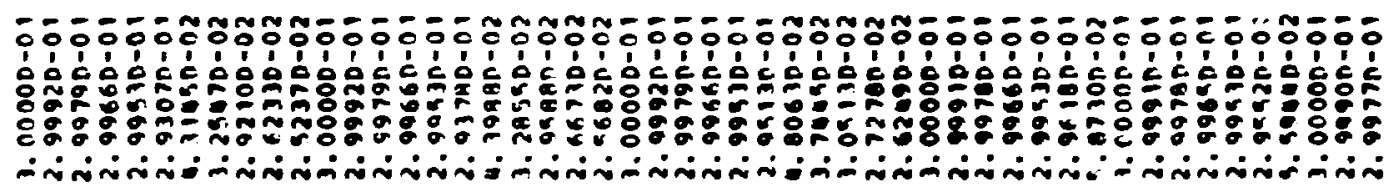

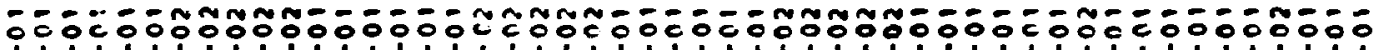

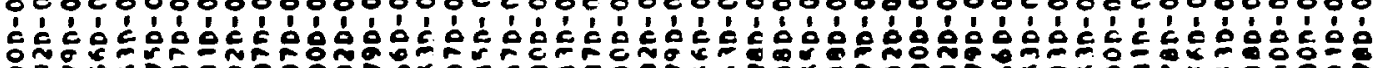

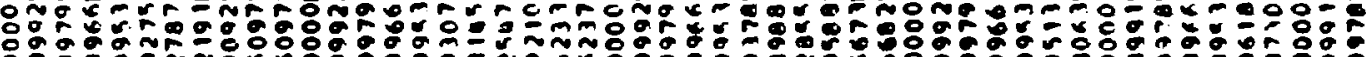

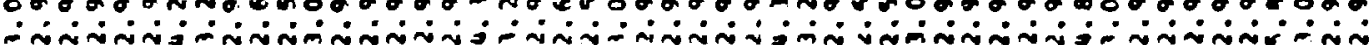




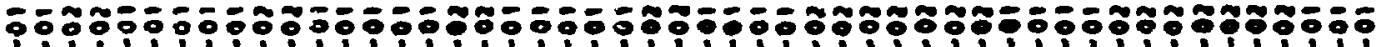

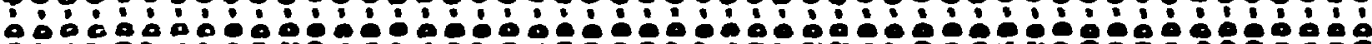

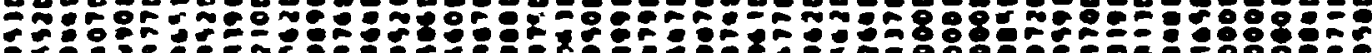

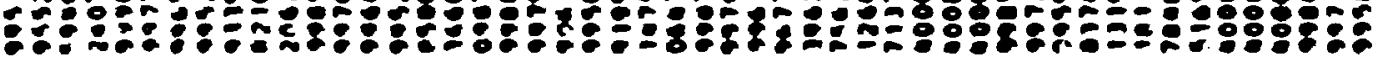

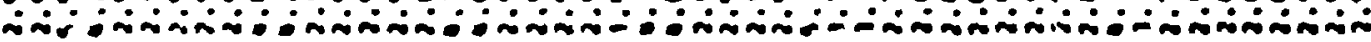
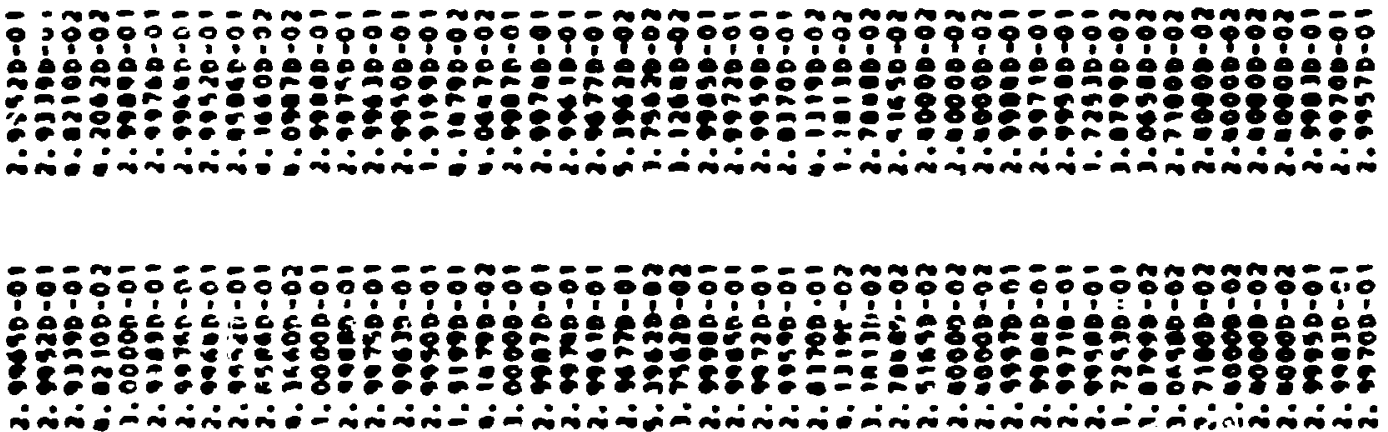

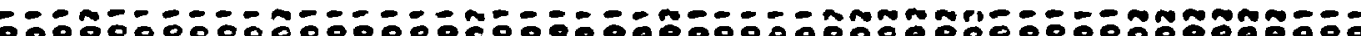

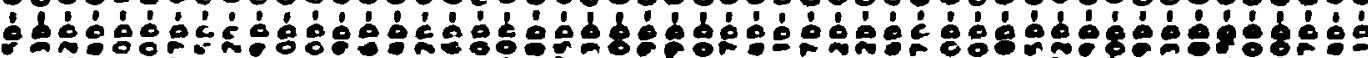

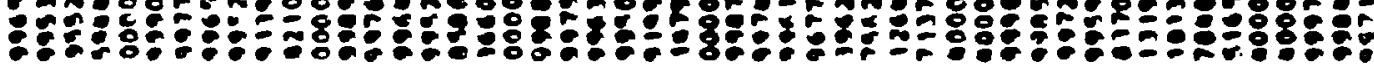

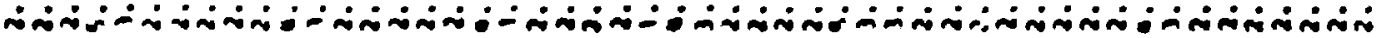

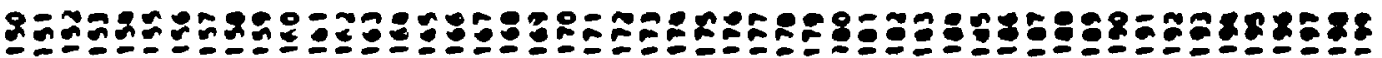




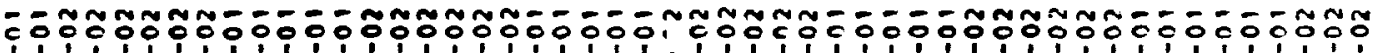

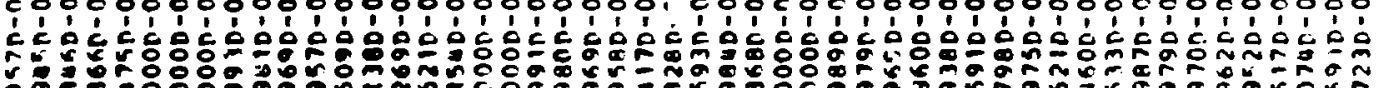

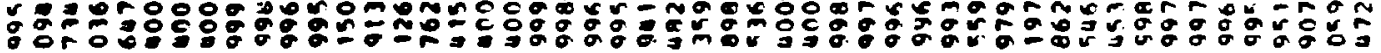

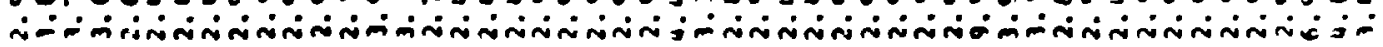

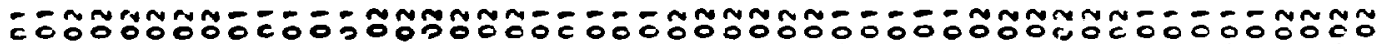

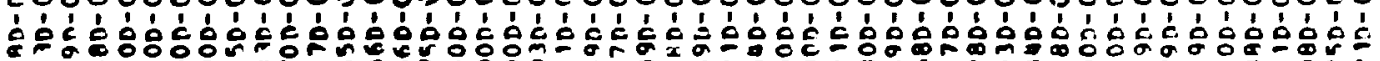

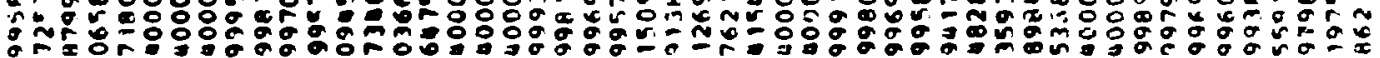

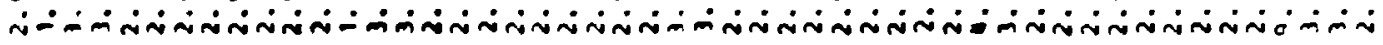

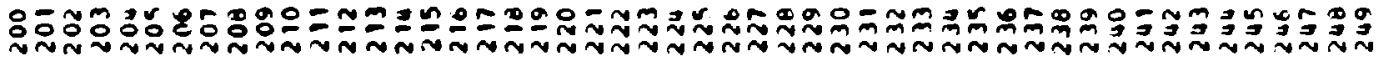




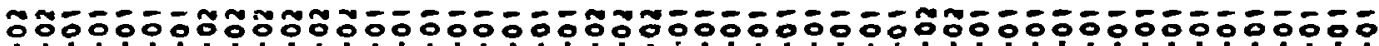

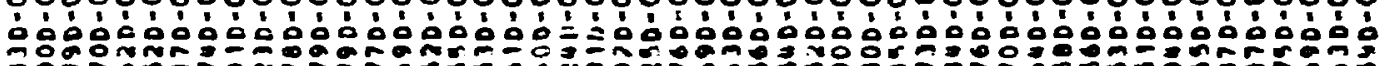

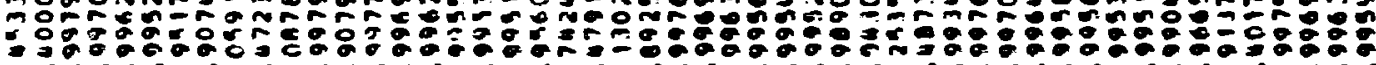

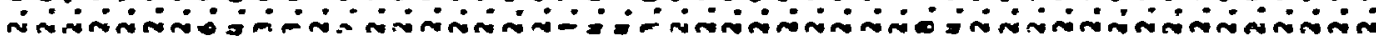
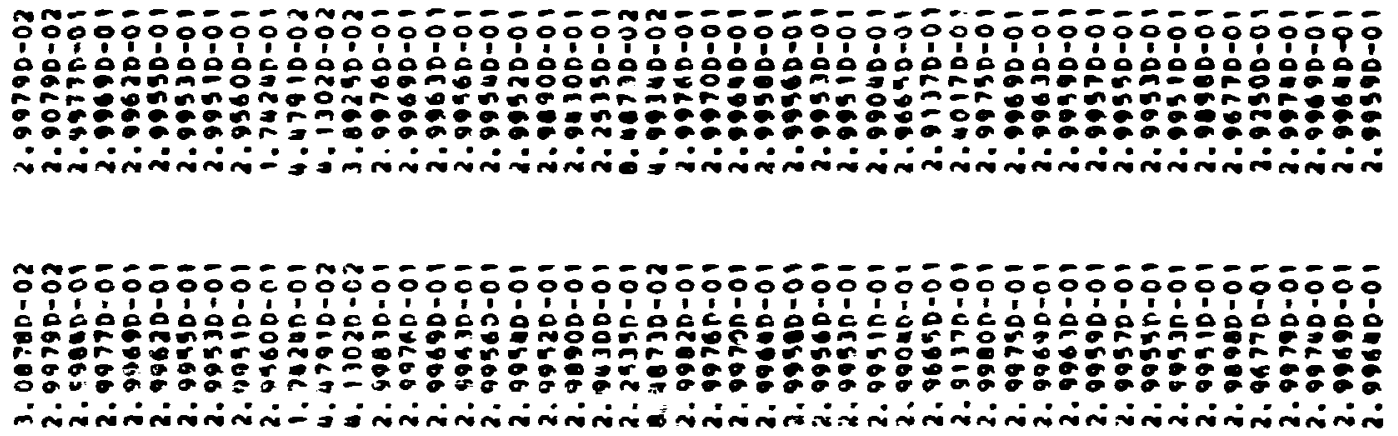

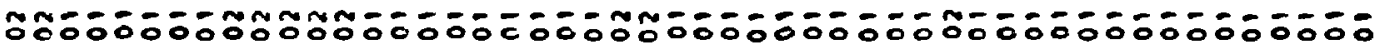

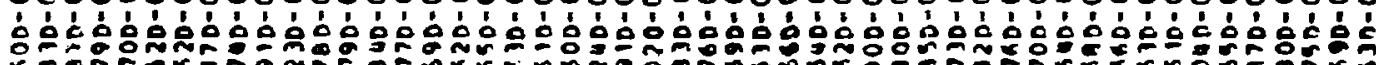

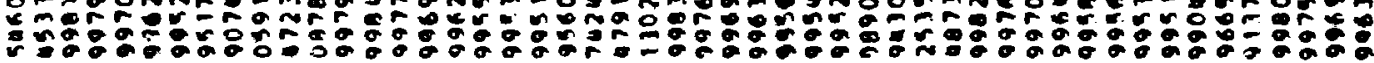

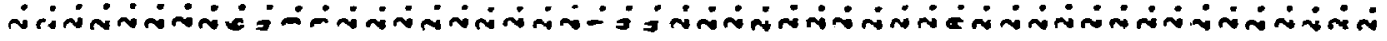

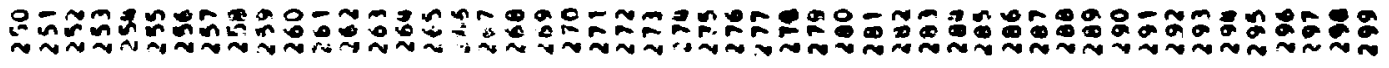


12
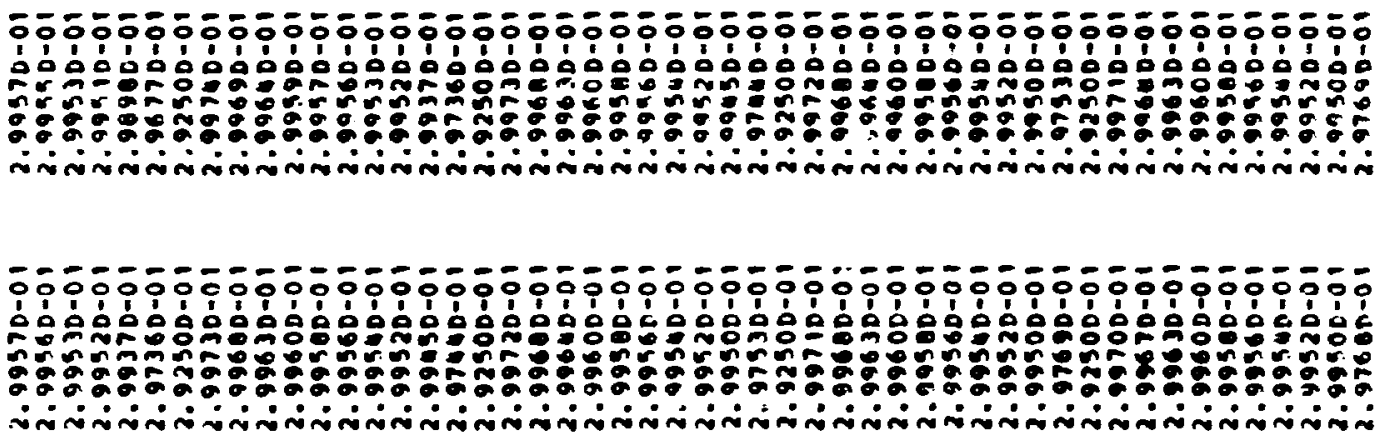

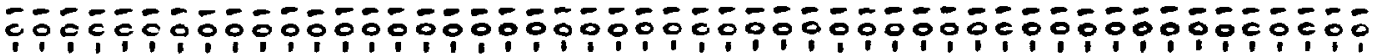

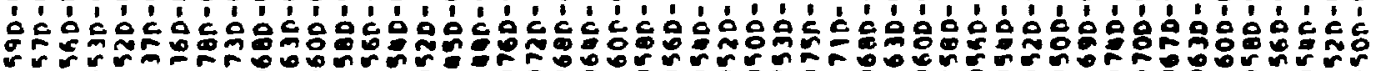

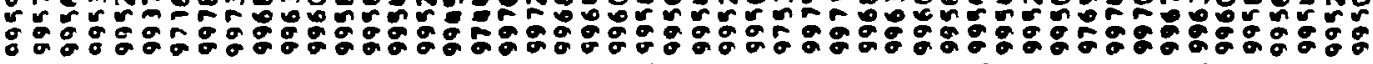

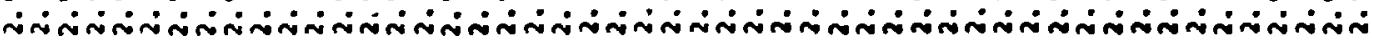

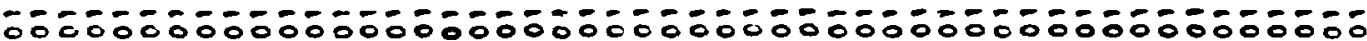

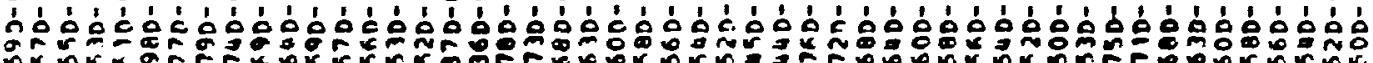

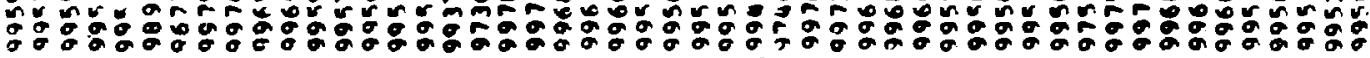

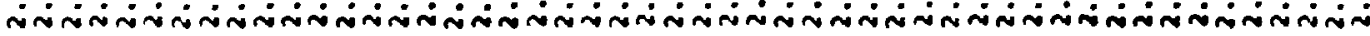

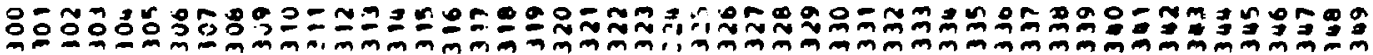




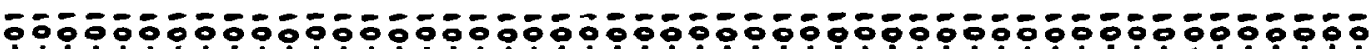

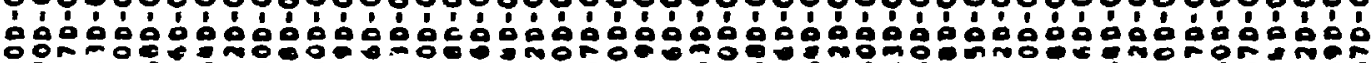

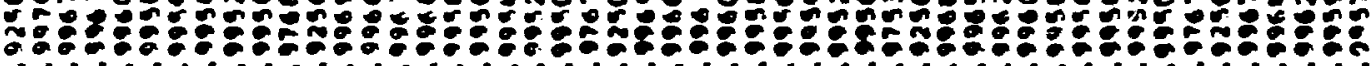

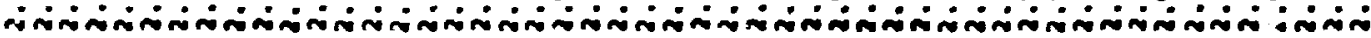
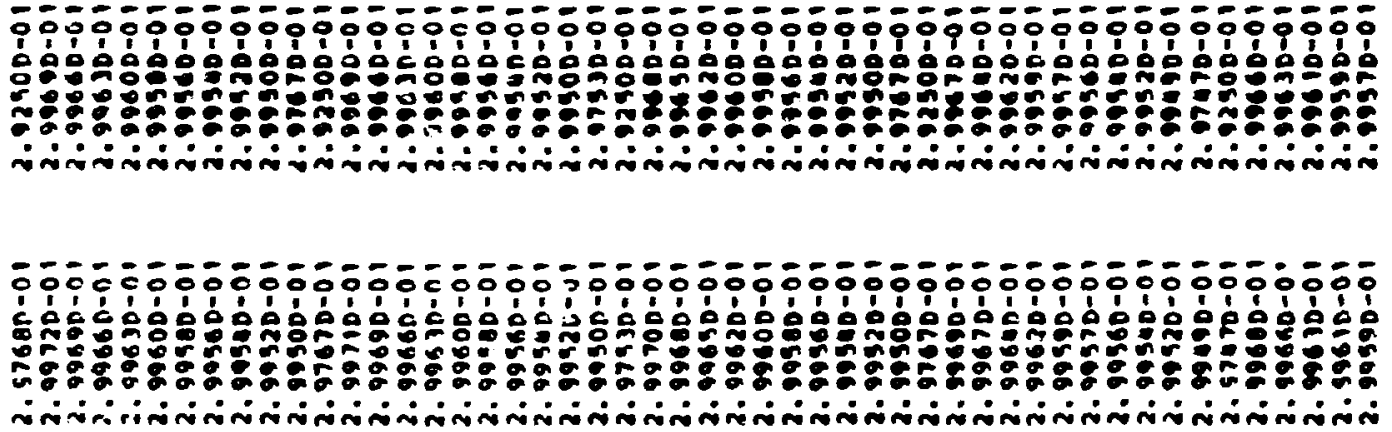

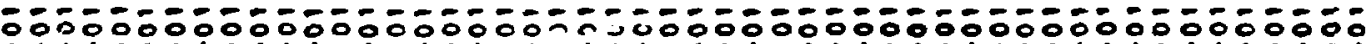

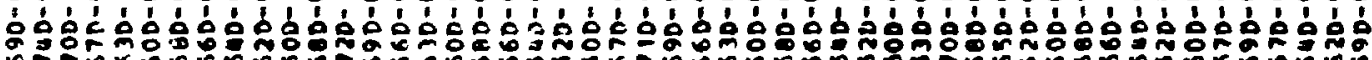

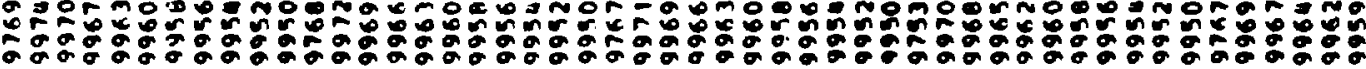

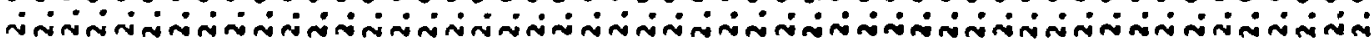

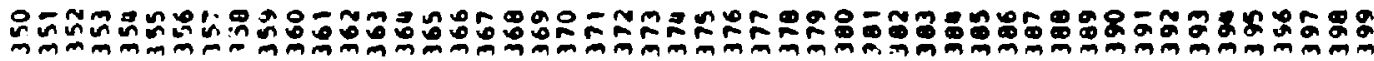




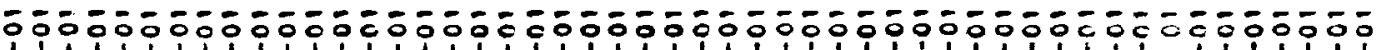

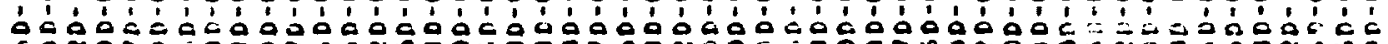

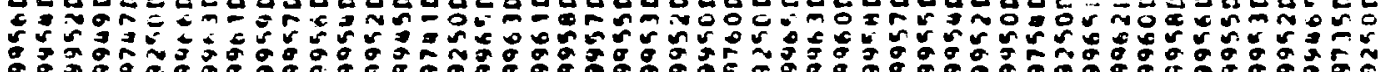

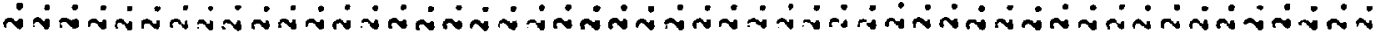

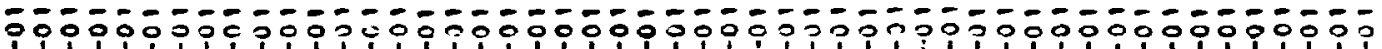

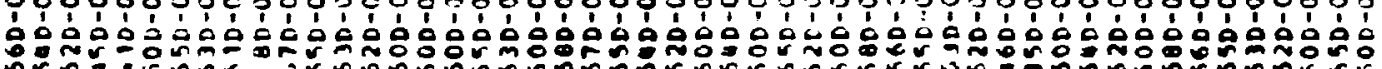

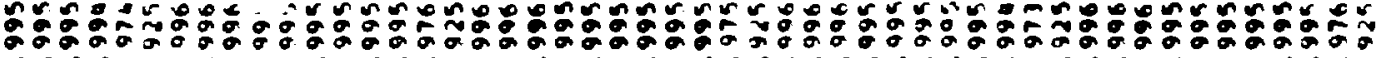

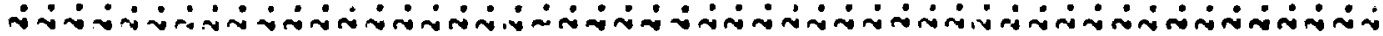

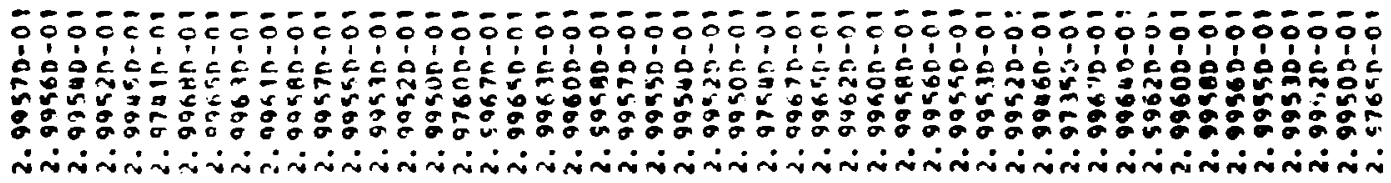

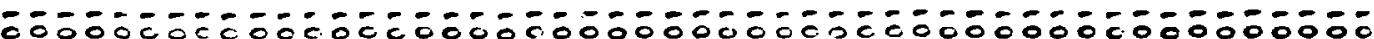

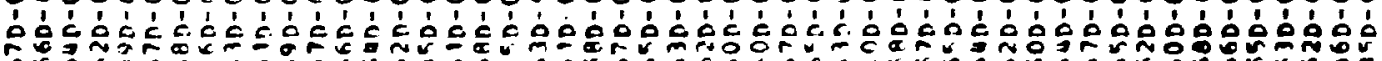

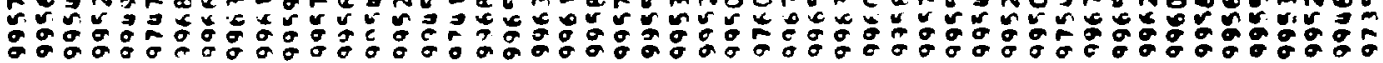

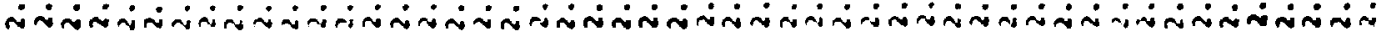




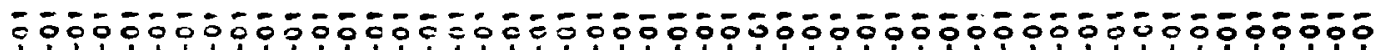

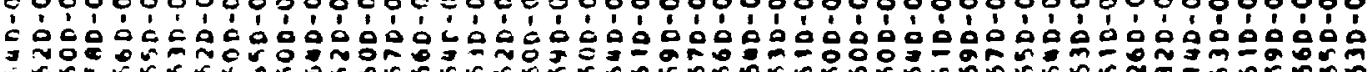

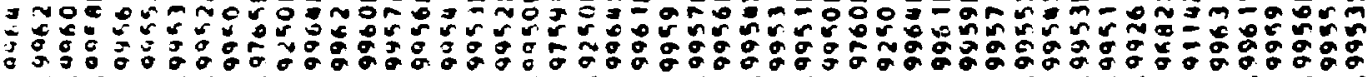

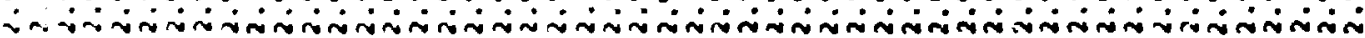

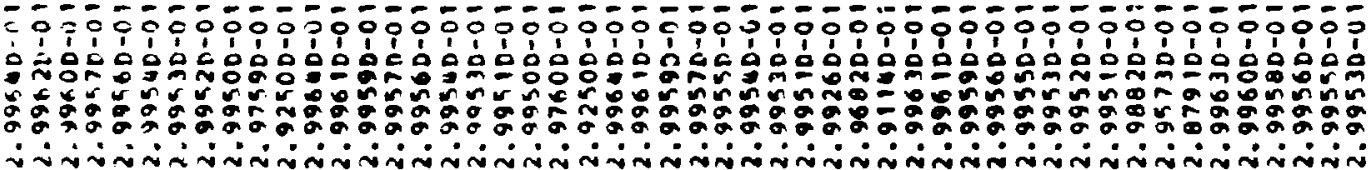

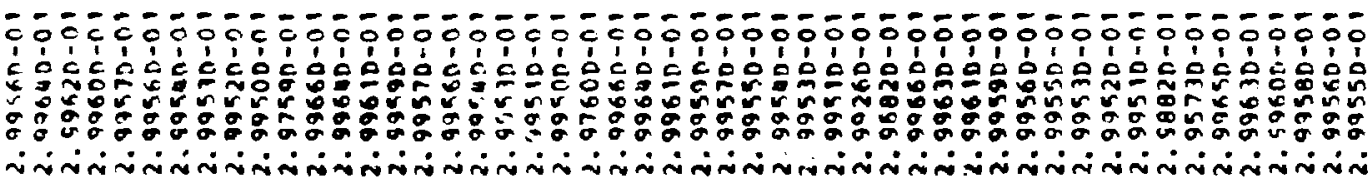

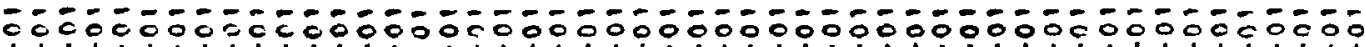

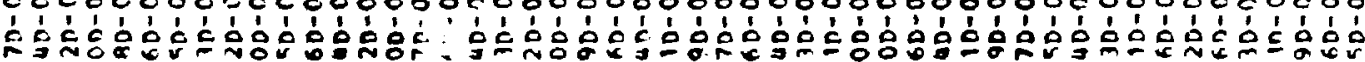

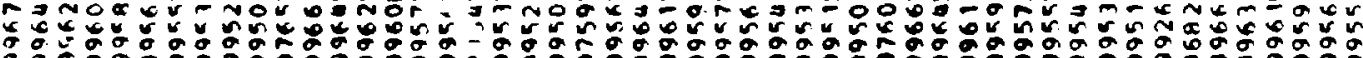

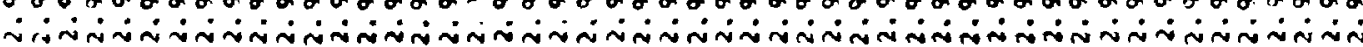




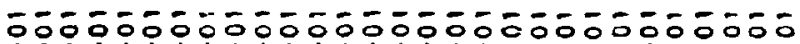

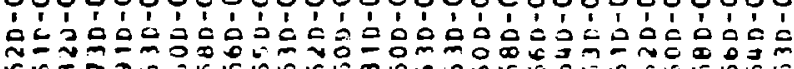

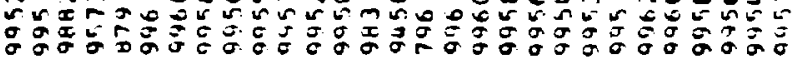

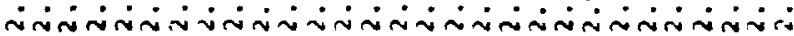

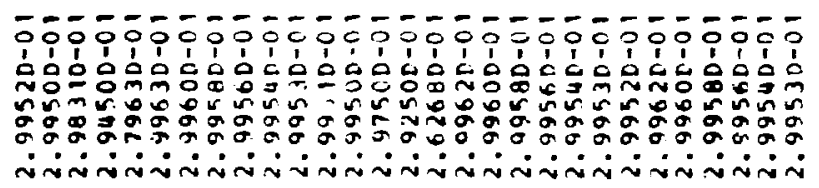

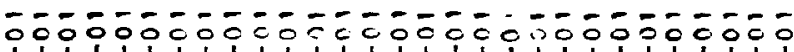

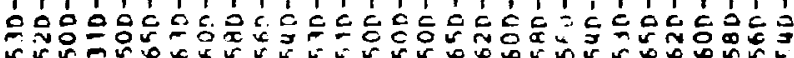

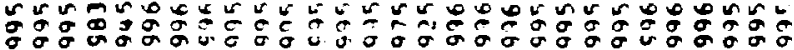

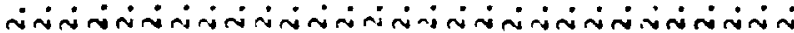

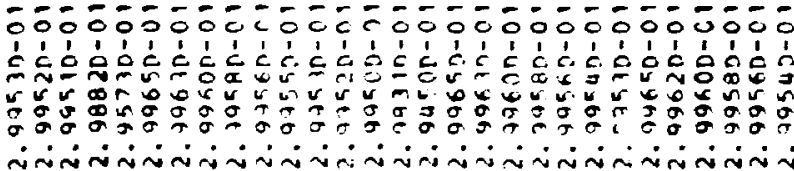

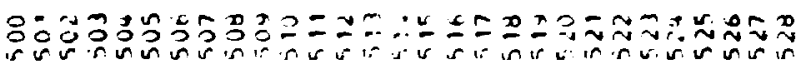




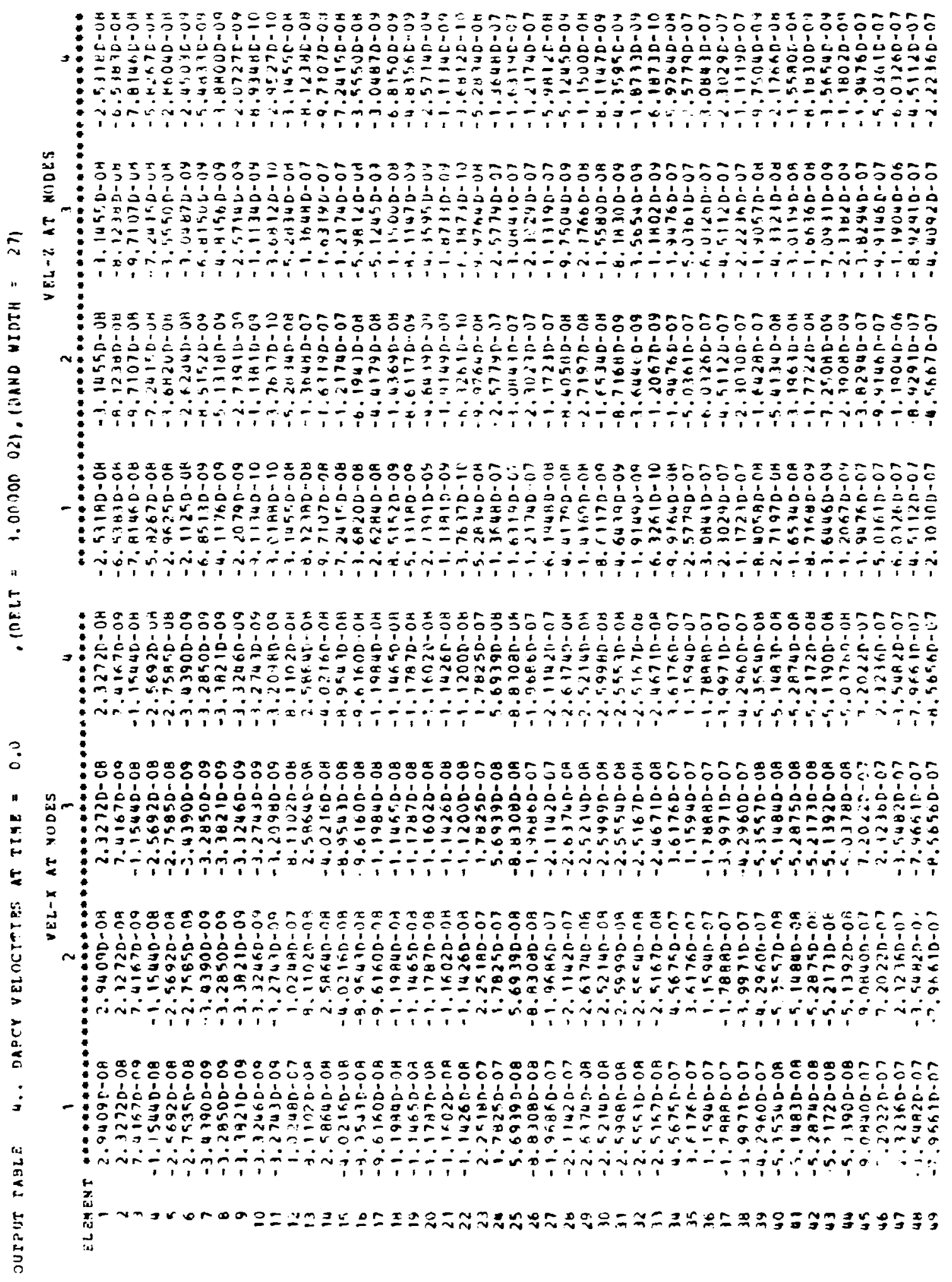




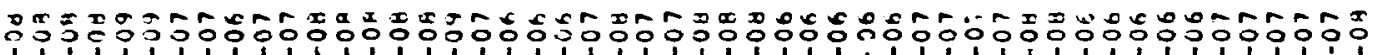

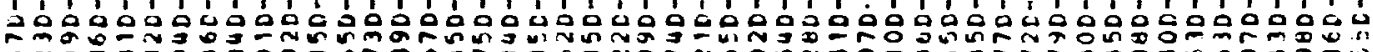

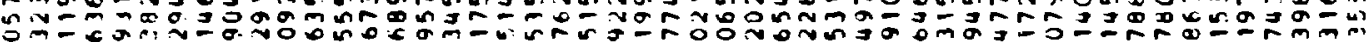

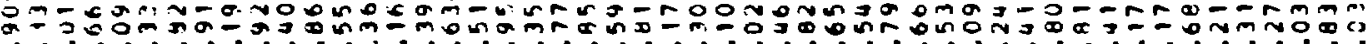

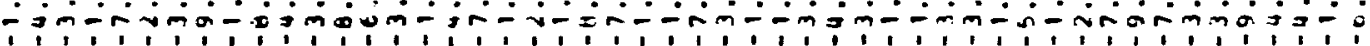

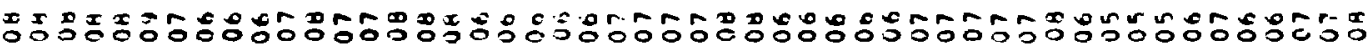

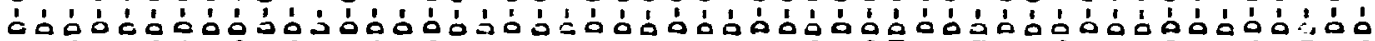

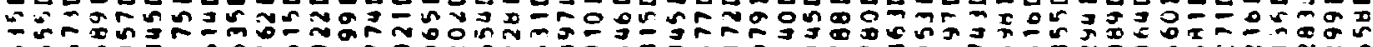

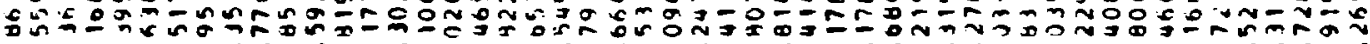

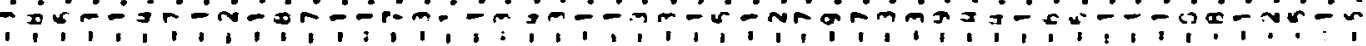

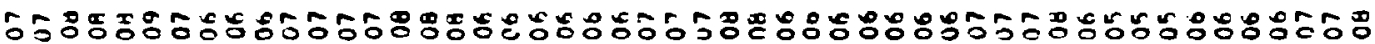

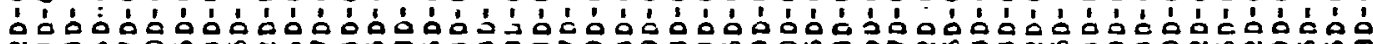

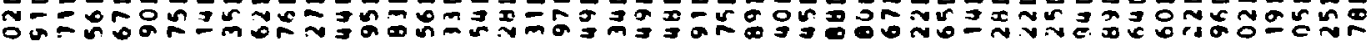

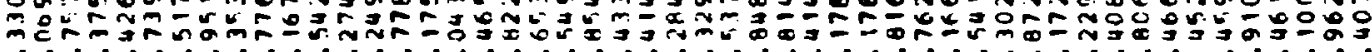

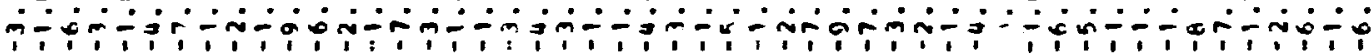

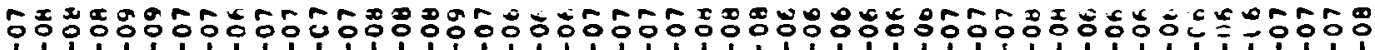

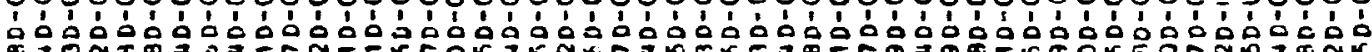

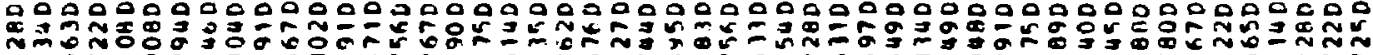

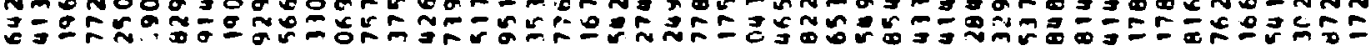

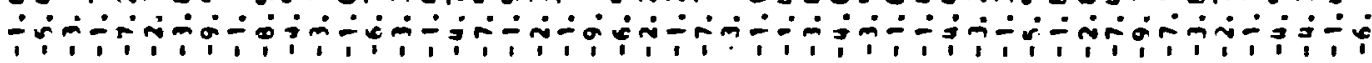

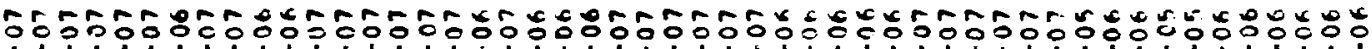

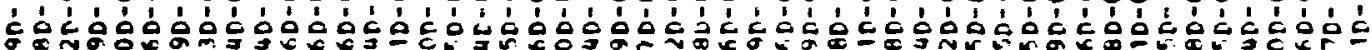

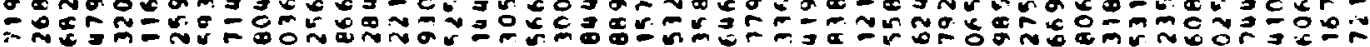

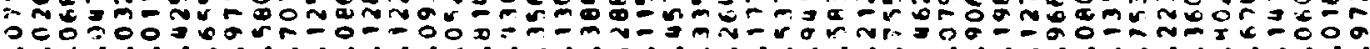

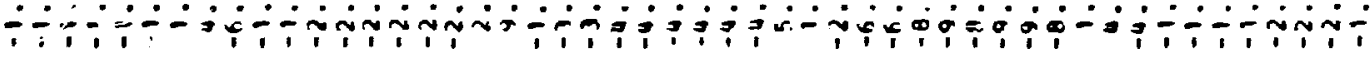

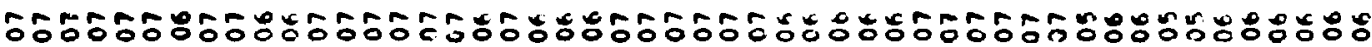

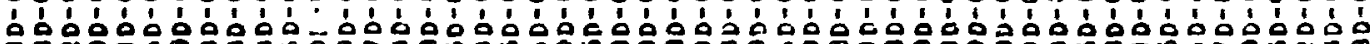

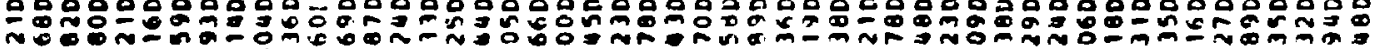

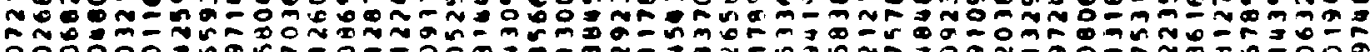

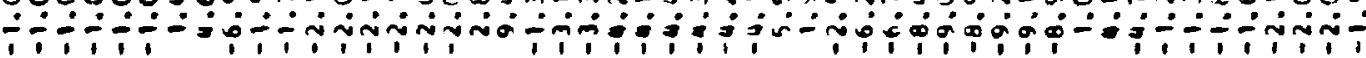

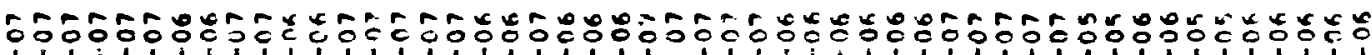

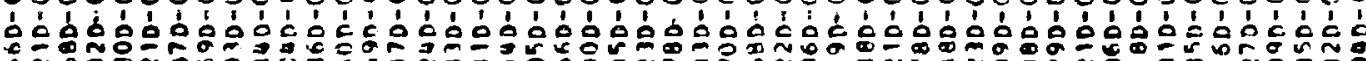
* No

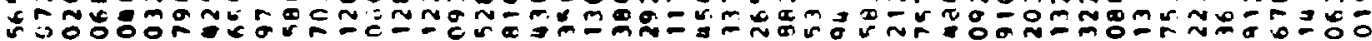

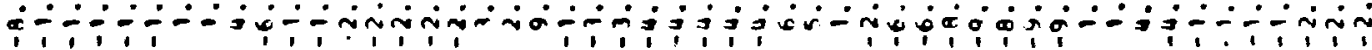

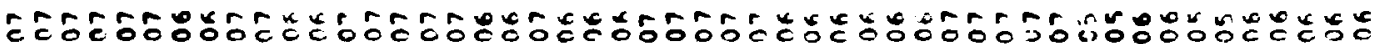

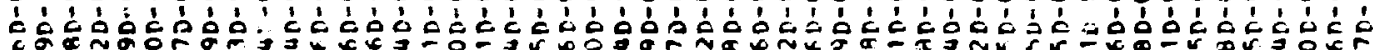

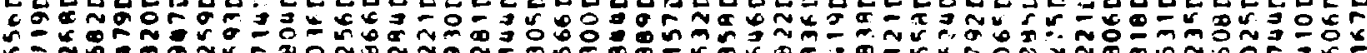

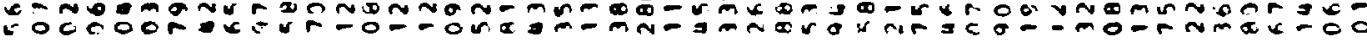

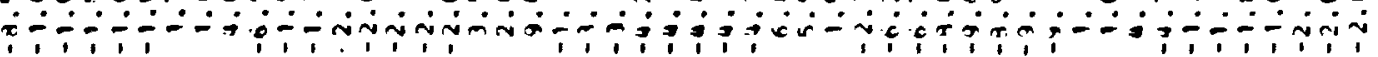

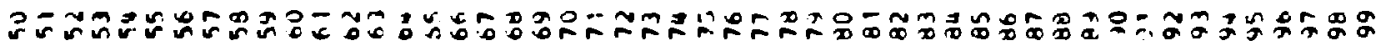




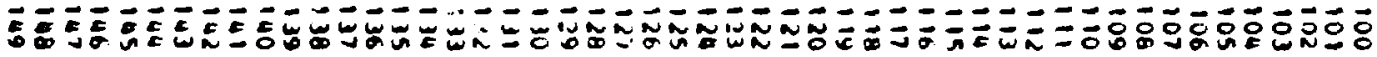

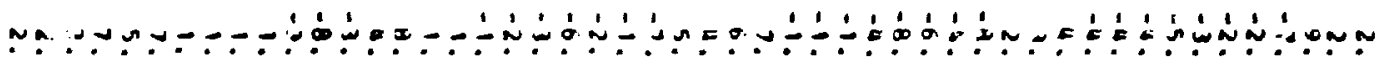
E

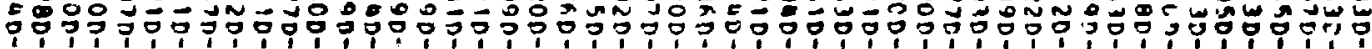

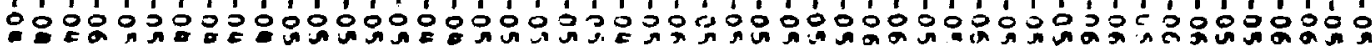

น น น

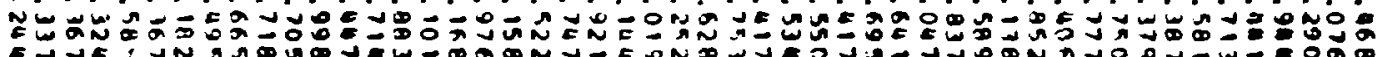

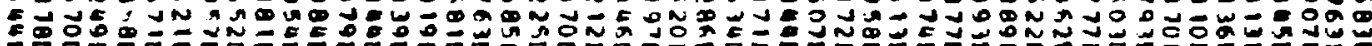

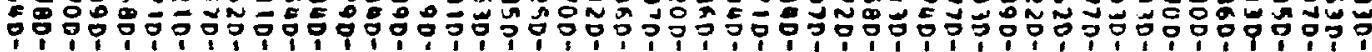

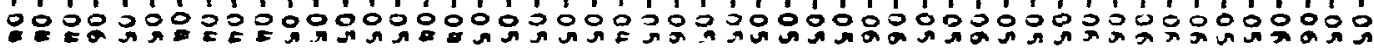

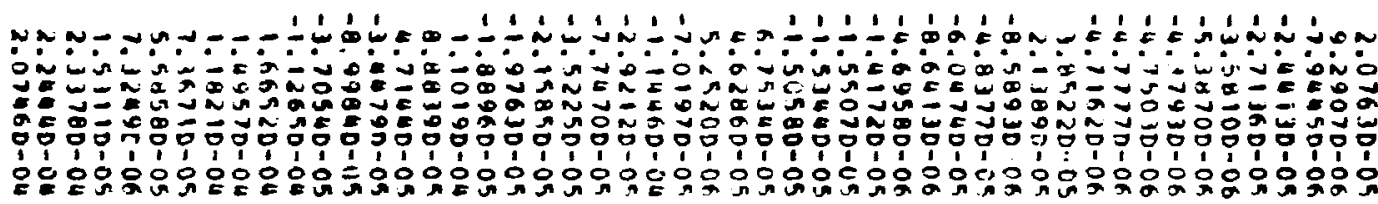

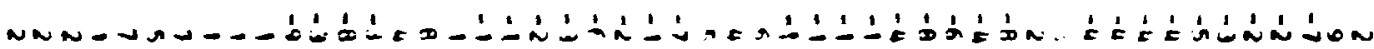

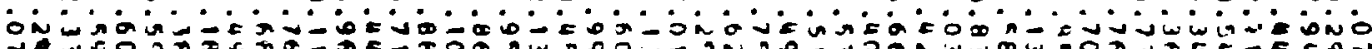
EE

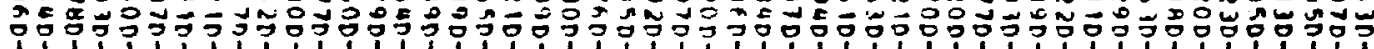

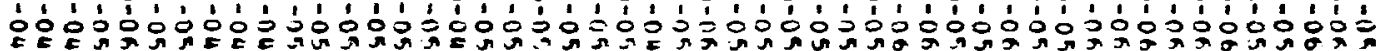

-1 -

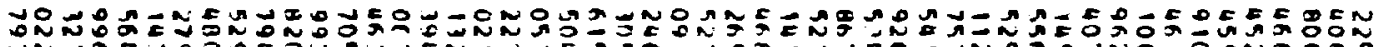

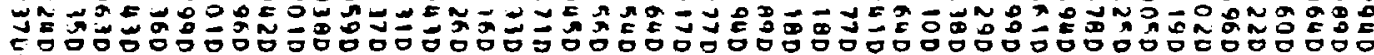

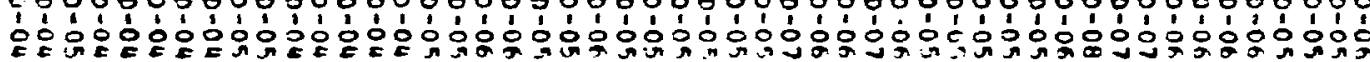

-

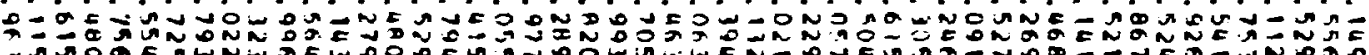

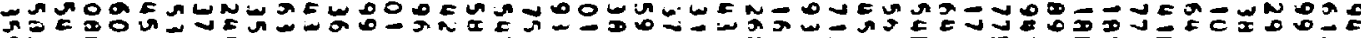

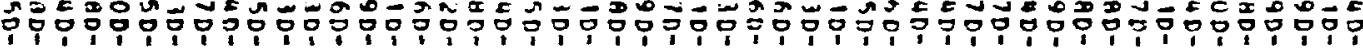

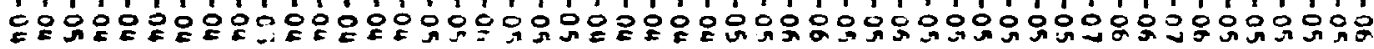

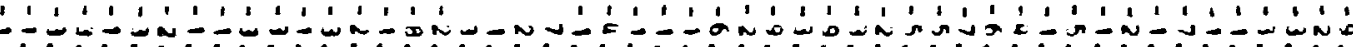

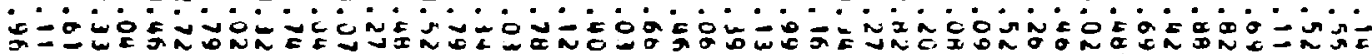

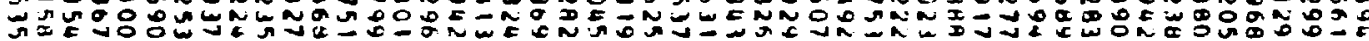

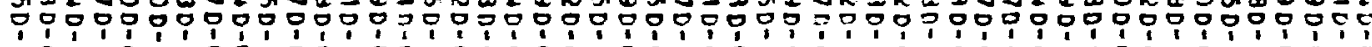

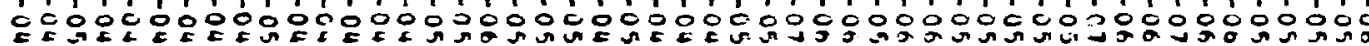

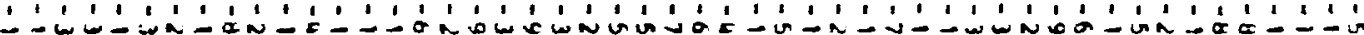

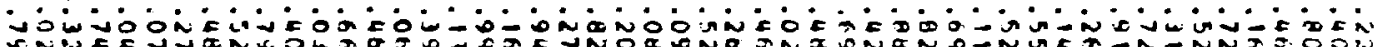

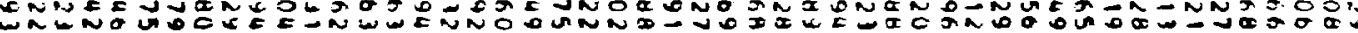

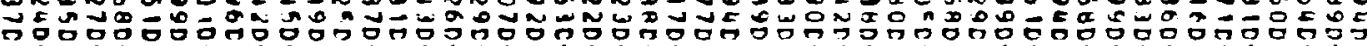

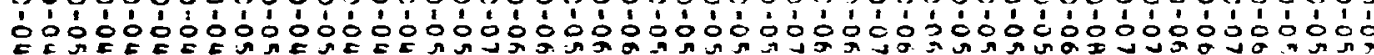


130

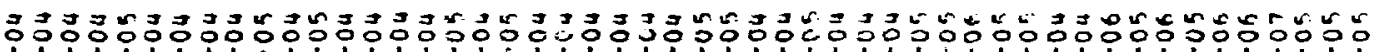

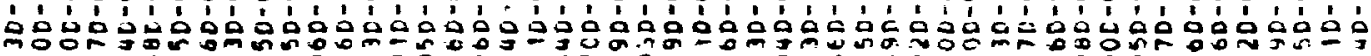

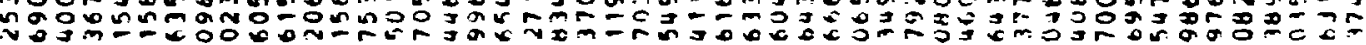

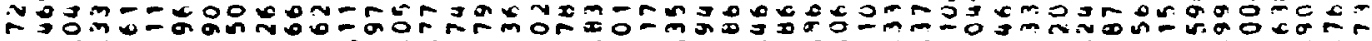

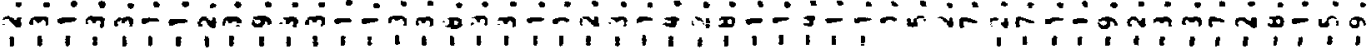

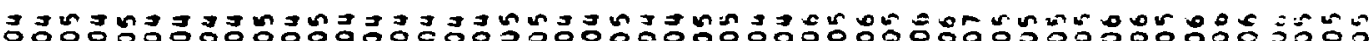

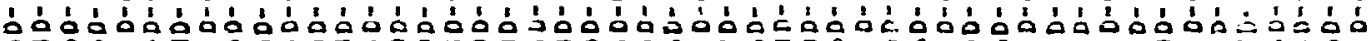

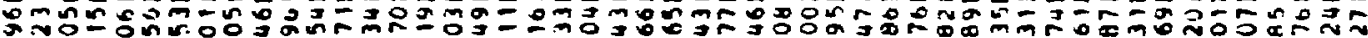

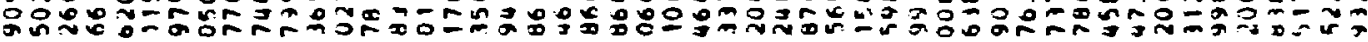
jodm-

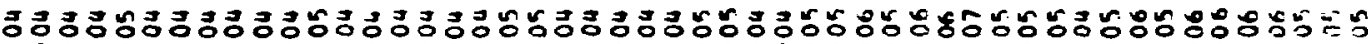

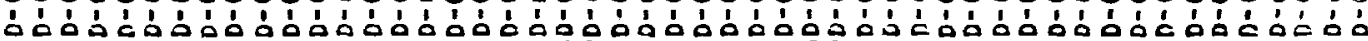

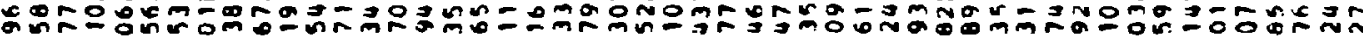

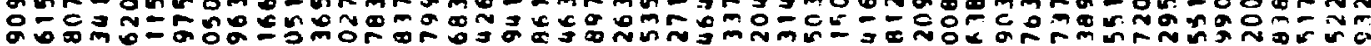

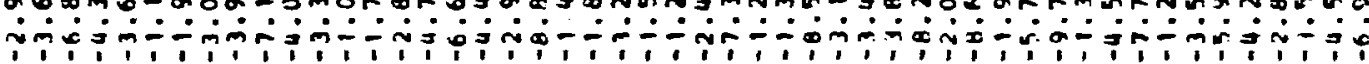

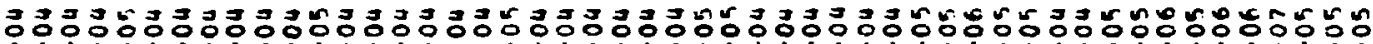

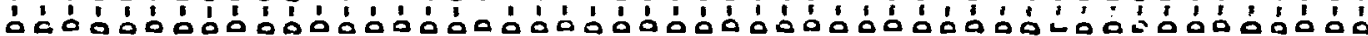

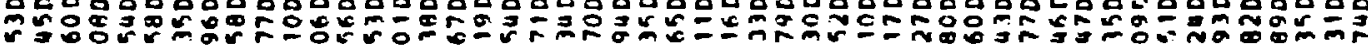

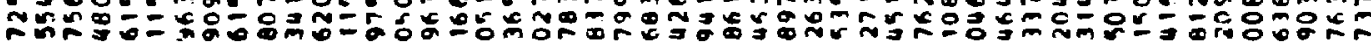

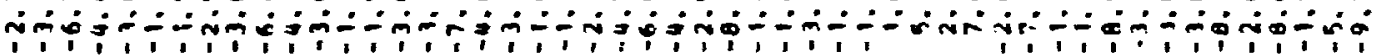

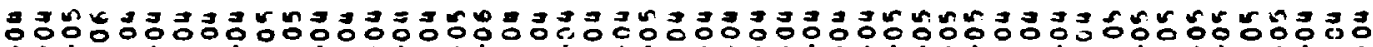

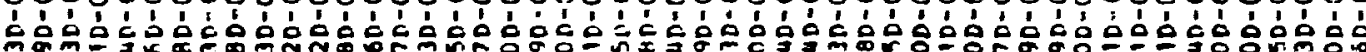
O을

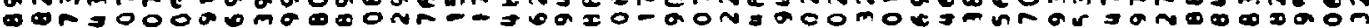

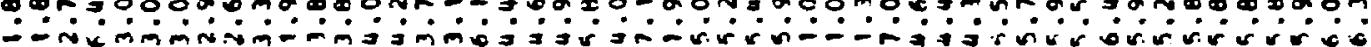

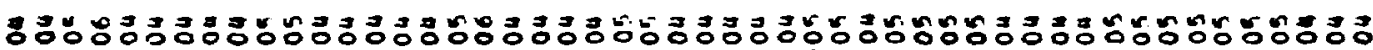

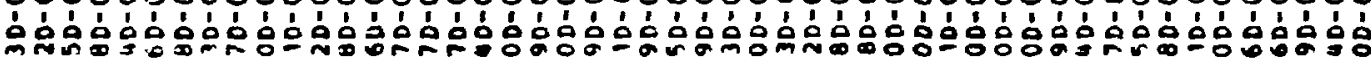
ำ

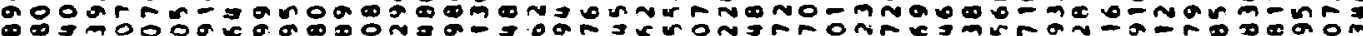

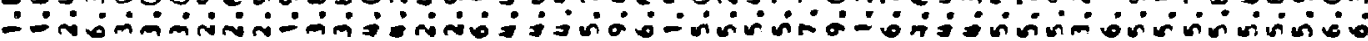

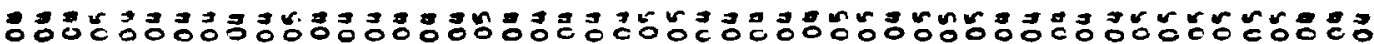

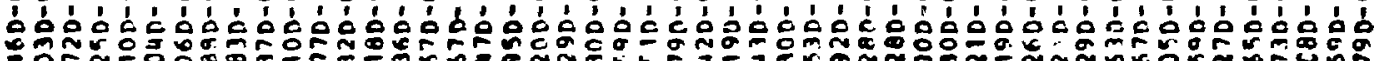

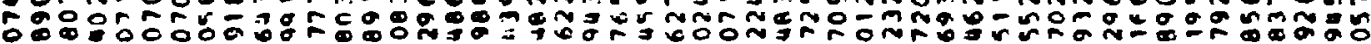

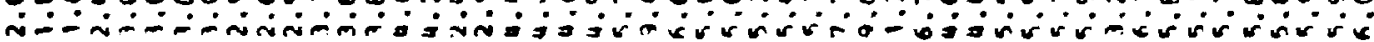

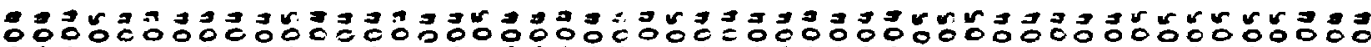

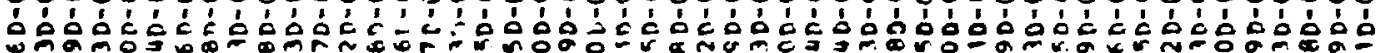

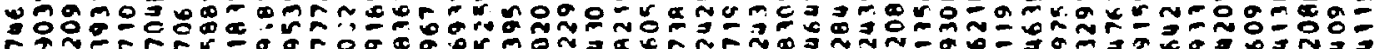
bror

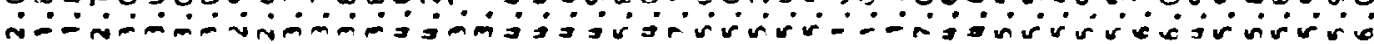

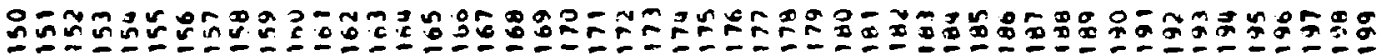




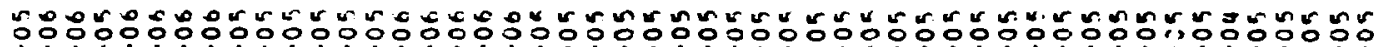

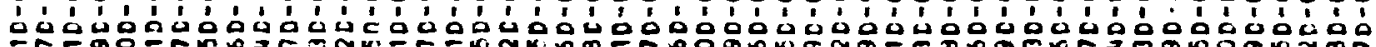

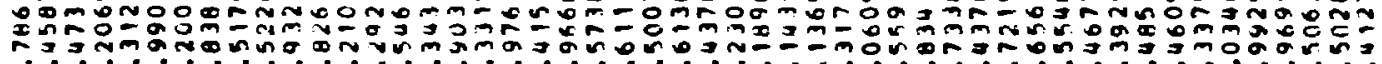

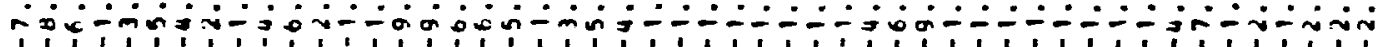

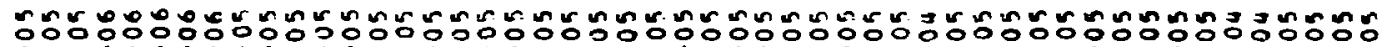

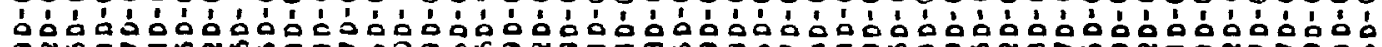

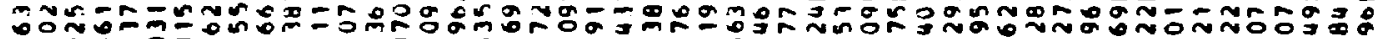

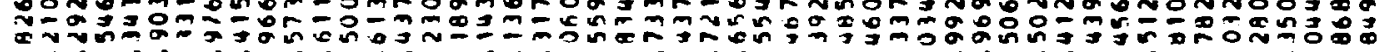

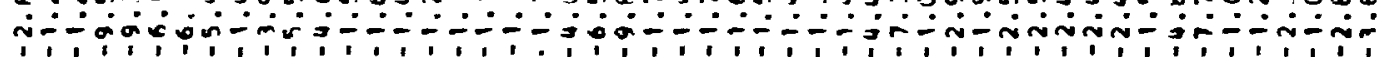

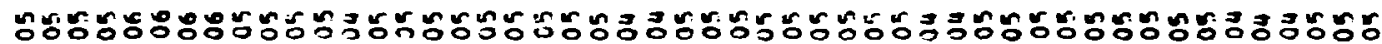

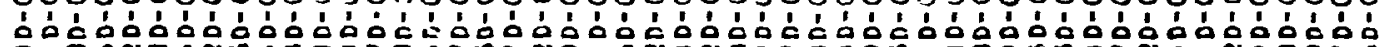

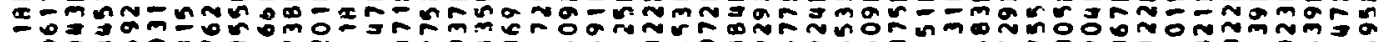

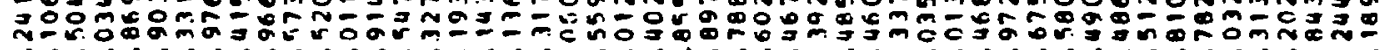

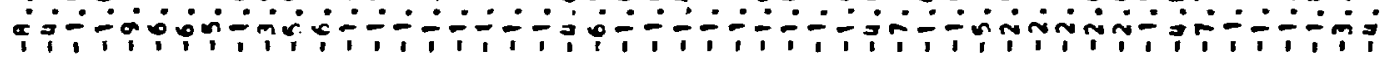

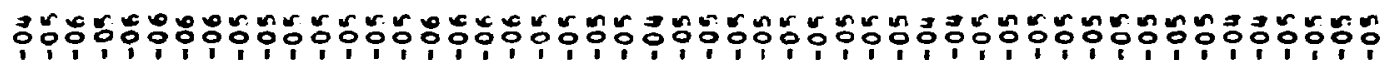

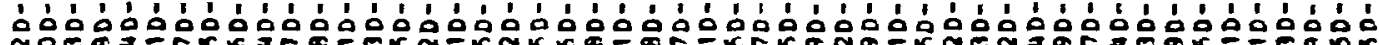

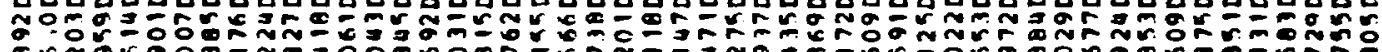

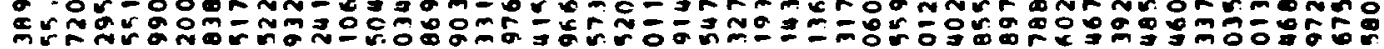

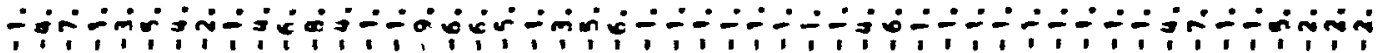

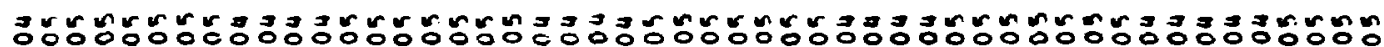

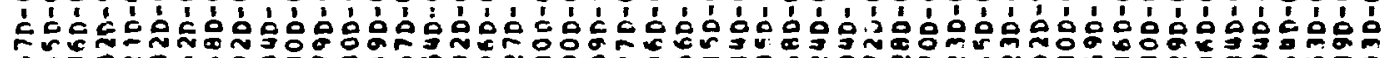

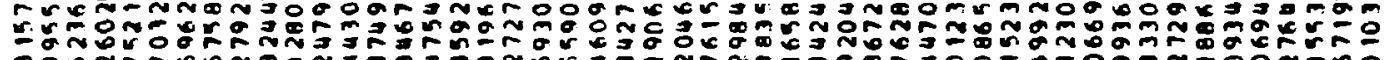

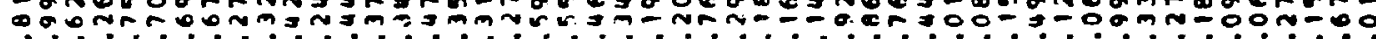

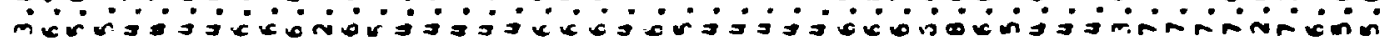

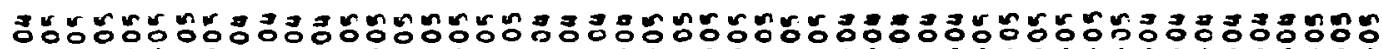

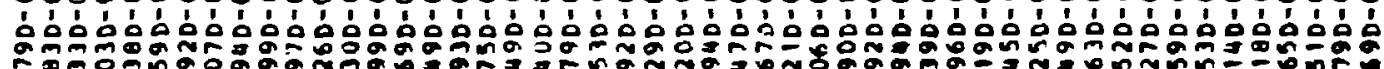

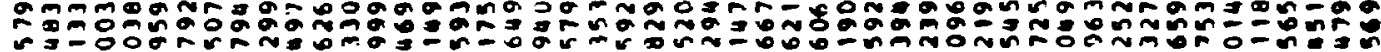

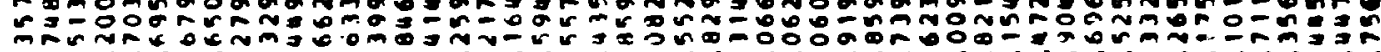
ions

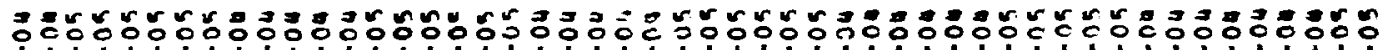

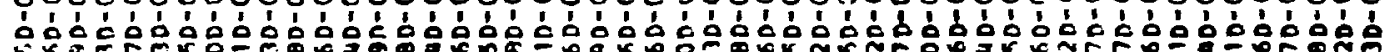

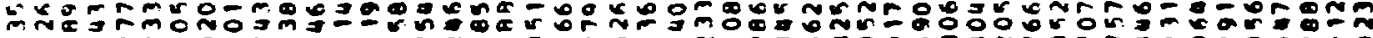

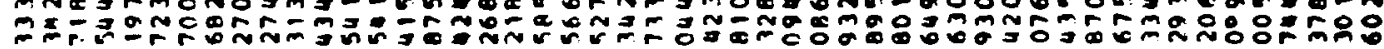

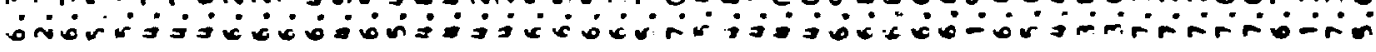

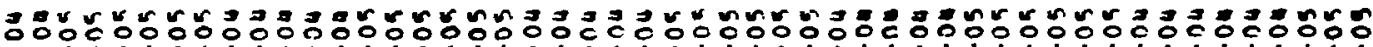

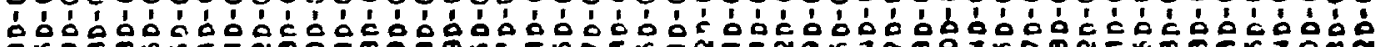

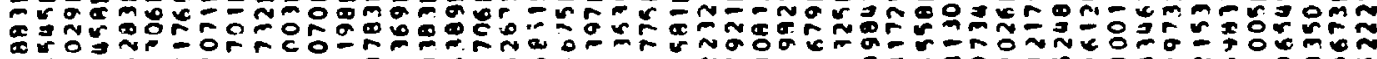

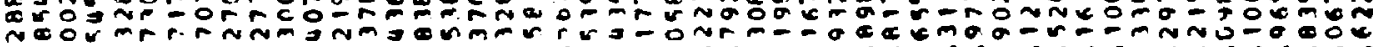

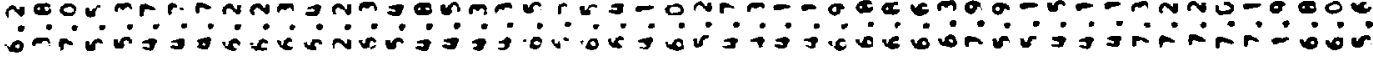

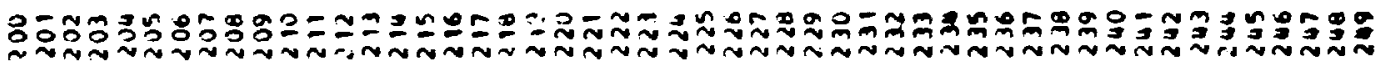




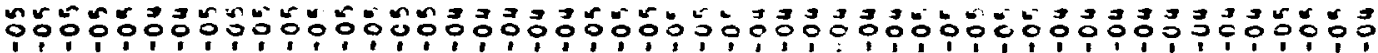

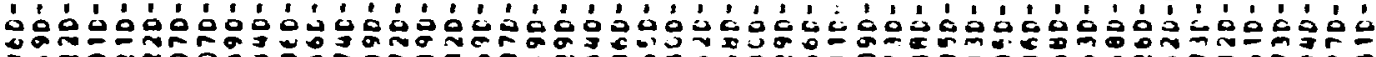

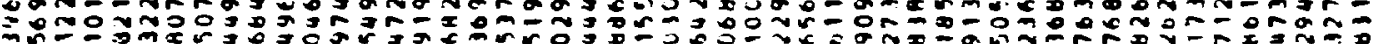

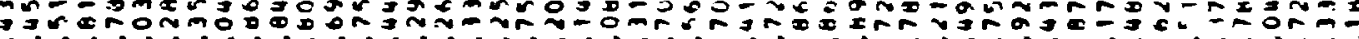

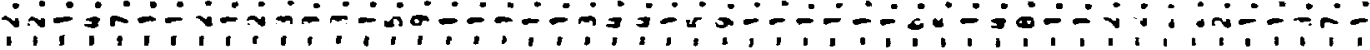

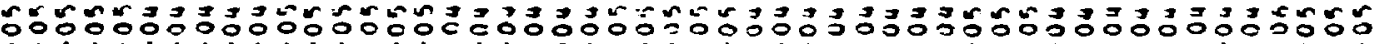

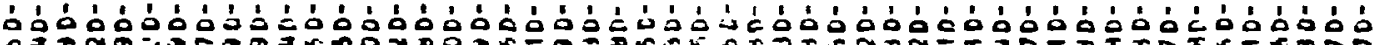

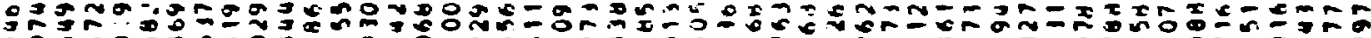

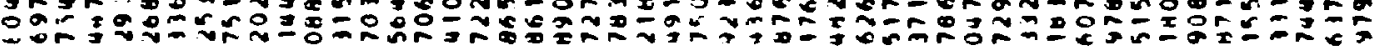

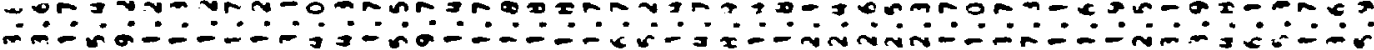

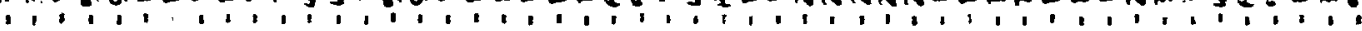

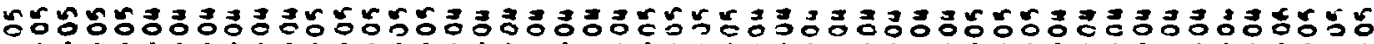

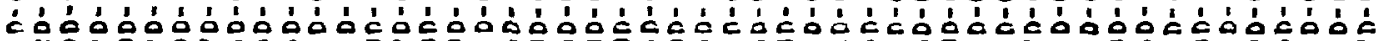

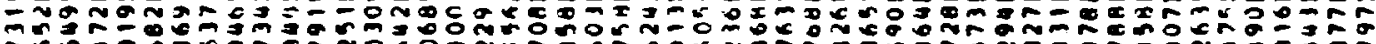

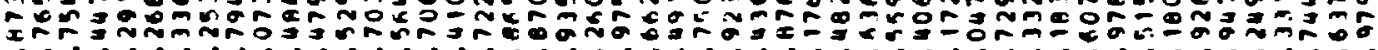

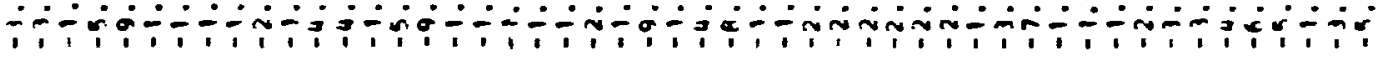

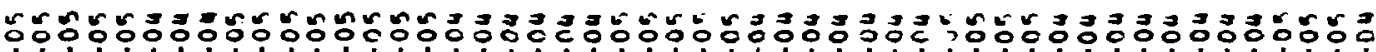

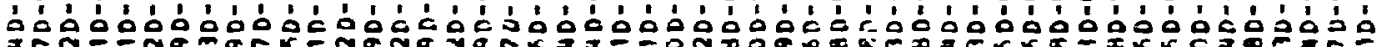

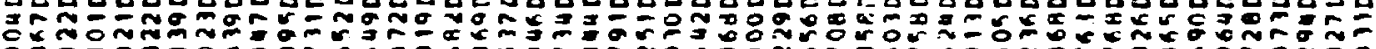

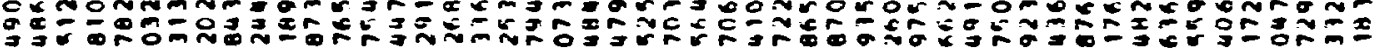

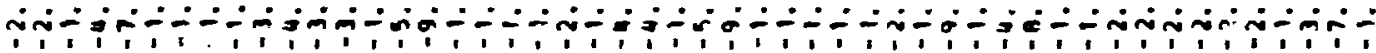

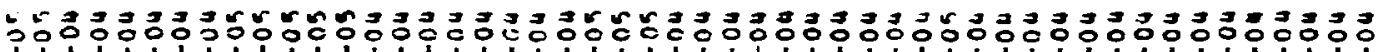

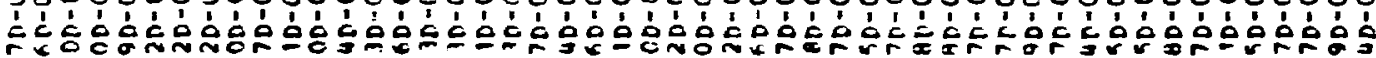

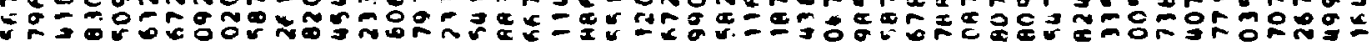

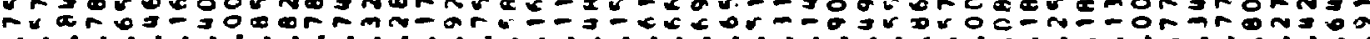

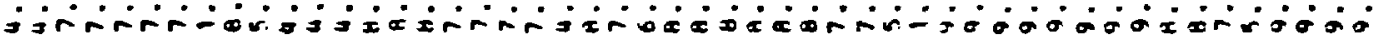

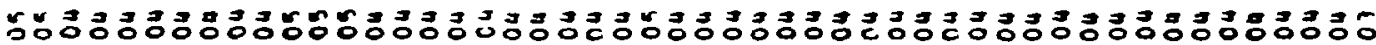

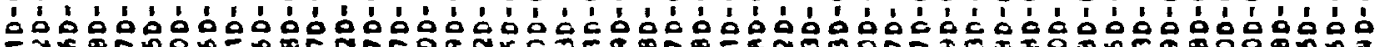

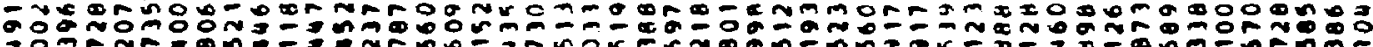

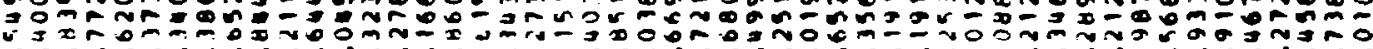

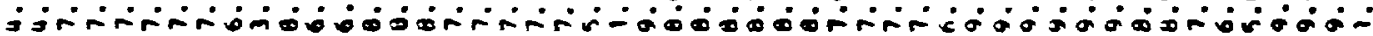

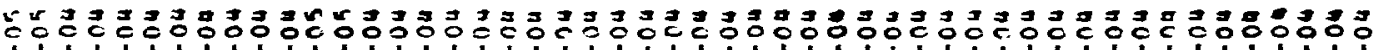

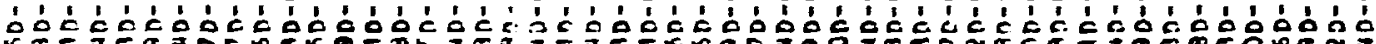

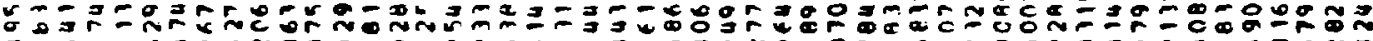

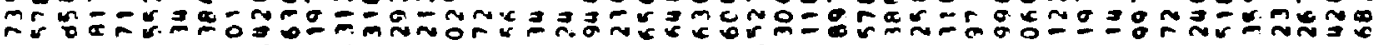

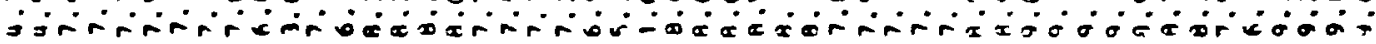

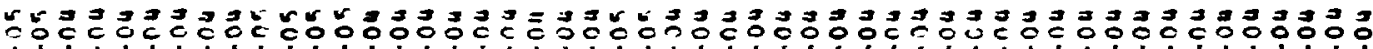

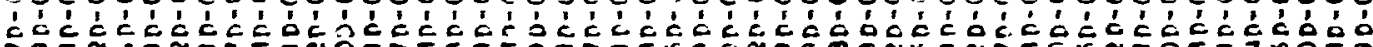

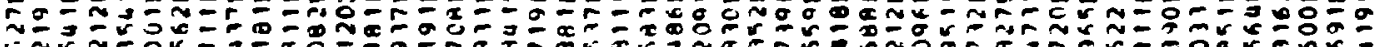

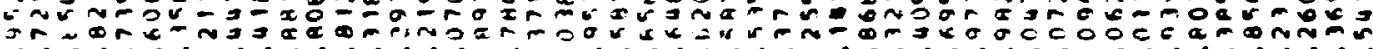

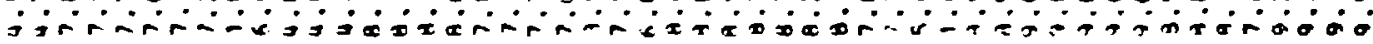

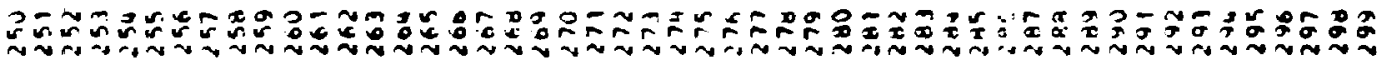




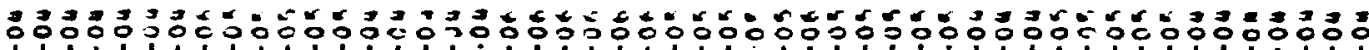

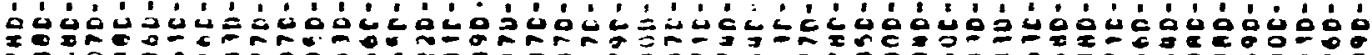

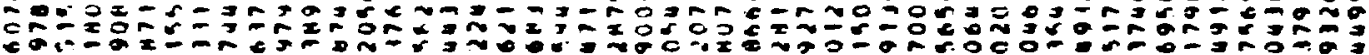

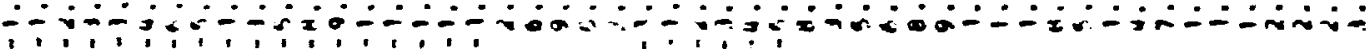

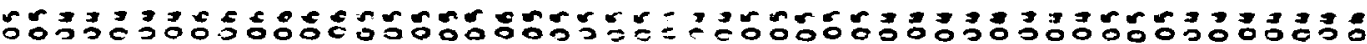

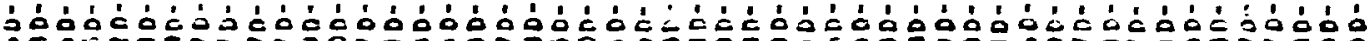

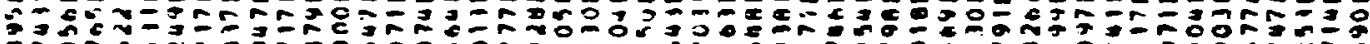

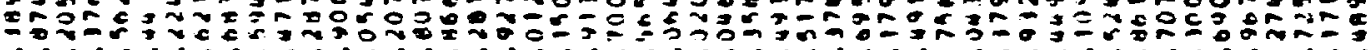

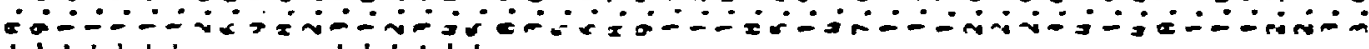

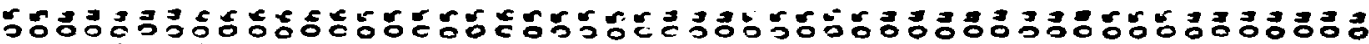

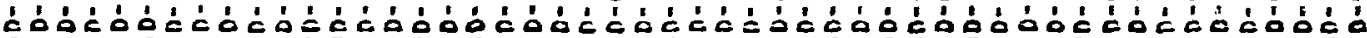

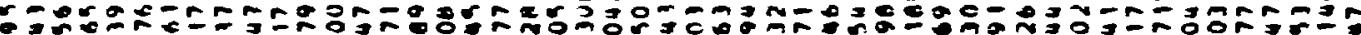

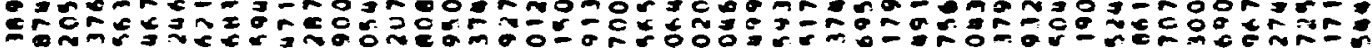

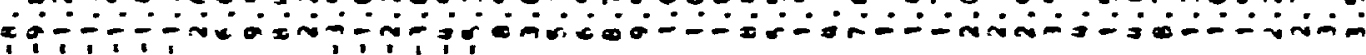

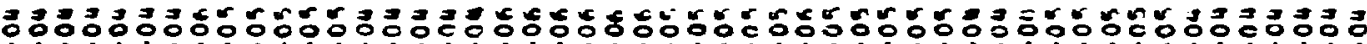

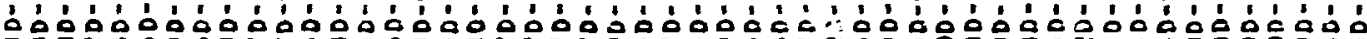

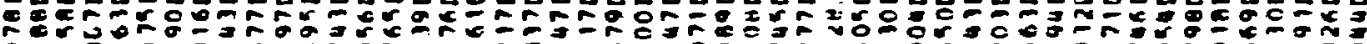

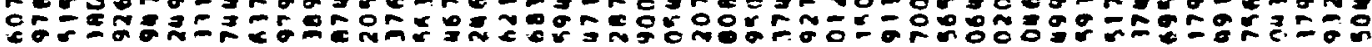

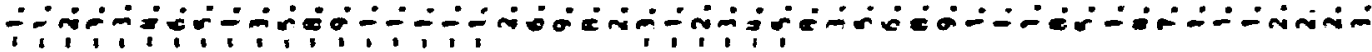

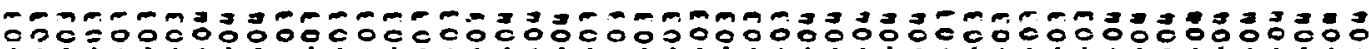

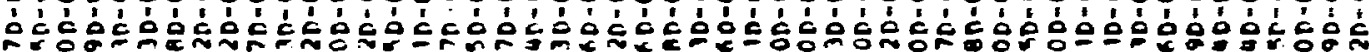

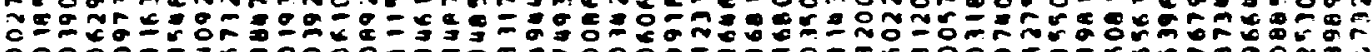

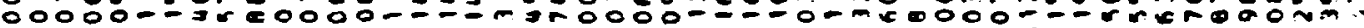

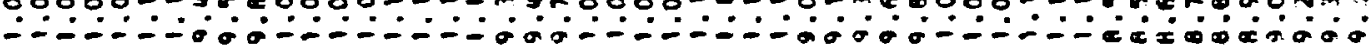

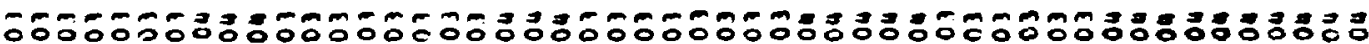

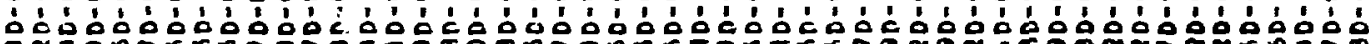

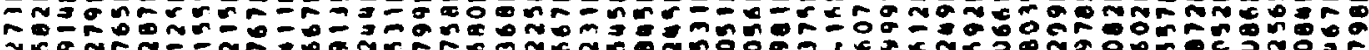

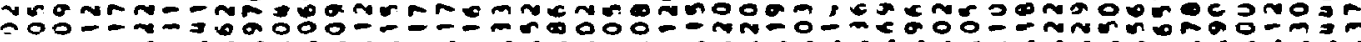

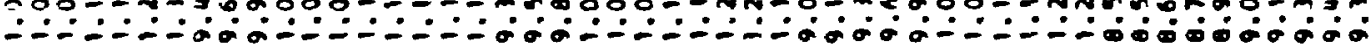

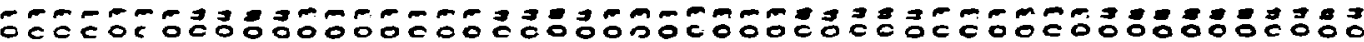

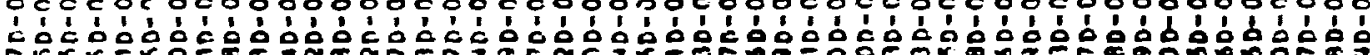

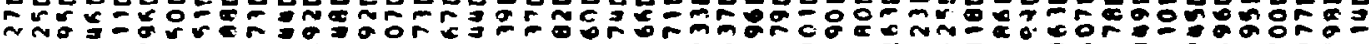

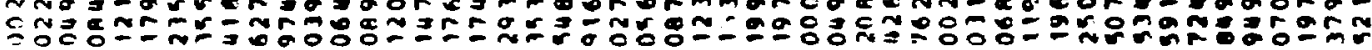

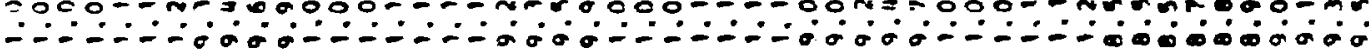

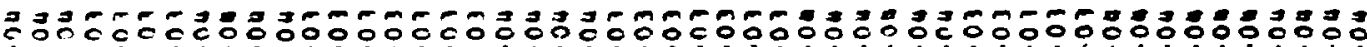

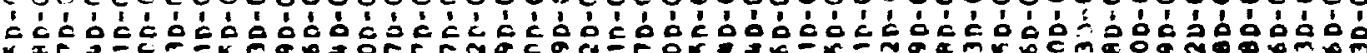

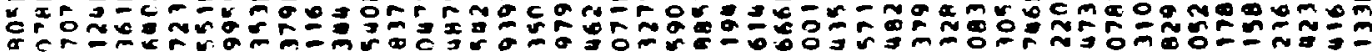

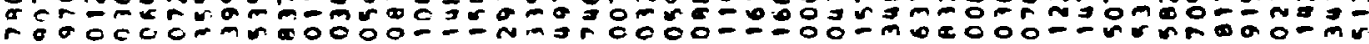

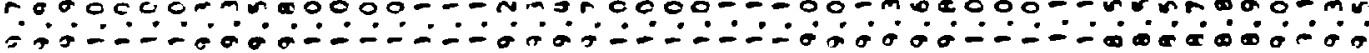

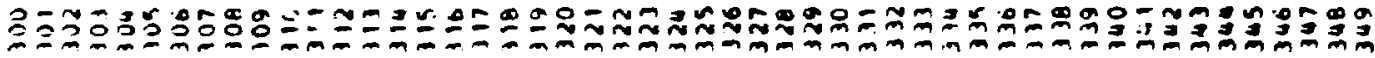




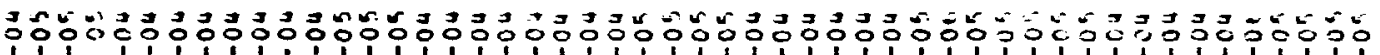

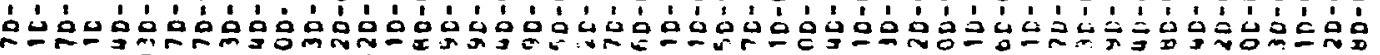

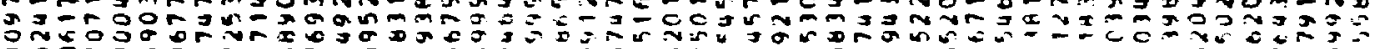

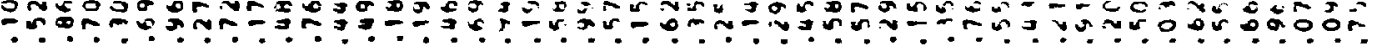

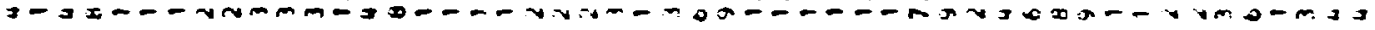

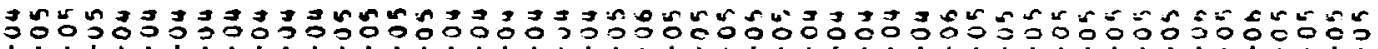

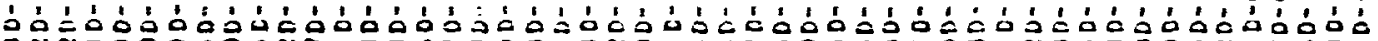
西

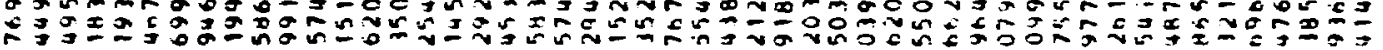
$\therefore$ -

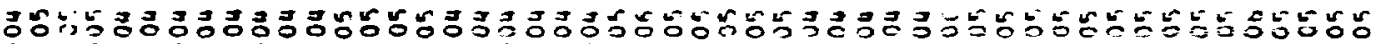

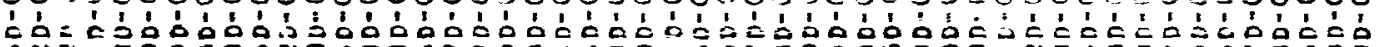

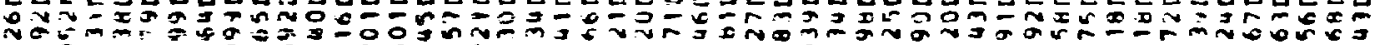

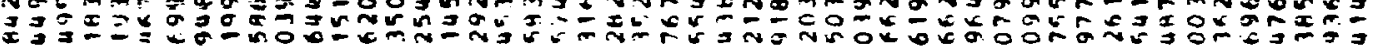
- ja:-

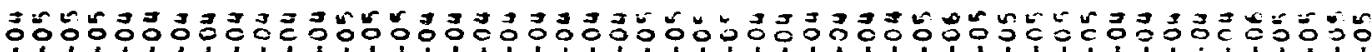

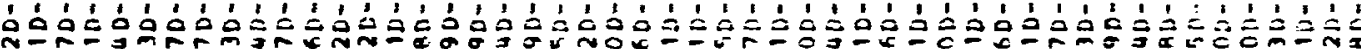

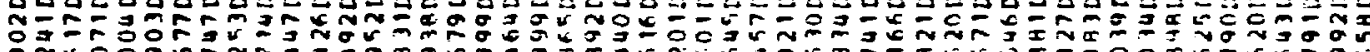

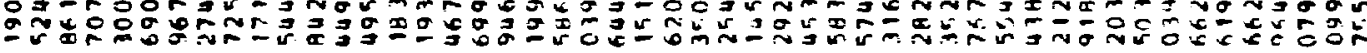

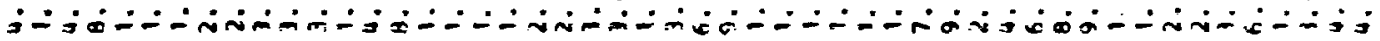

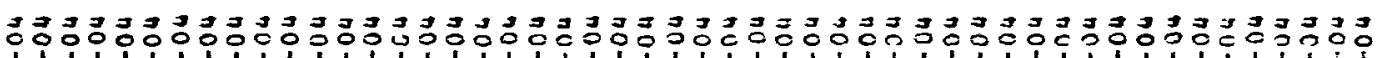

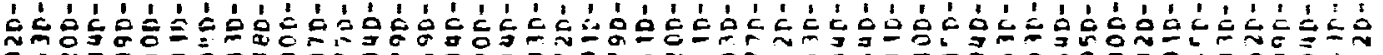

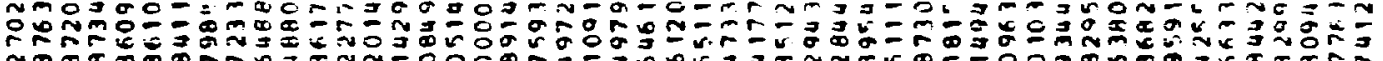

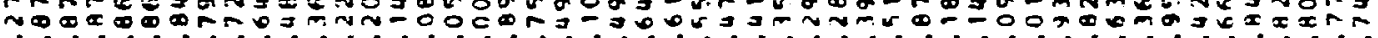

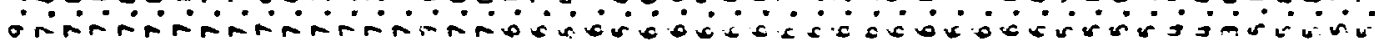

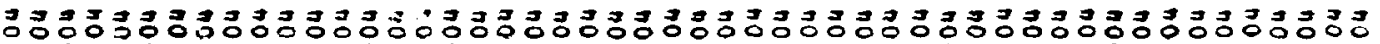

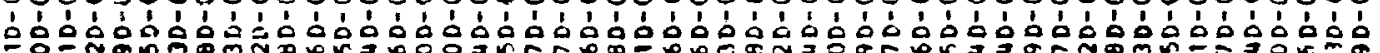

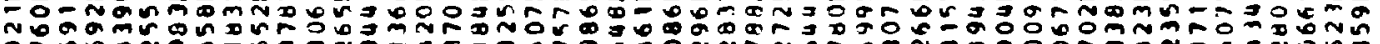
Tơ

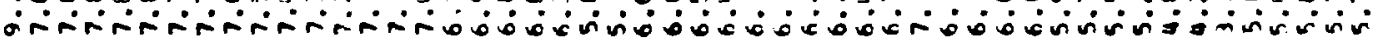

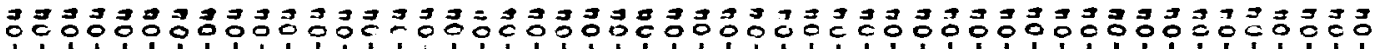

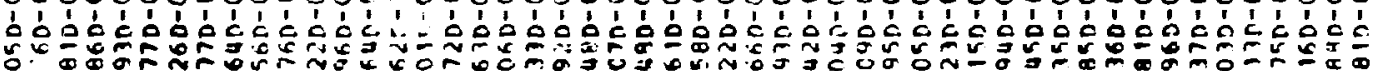
等

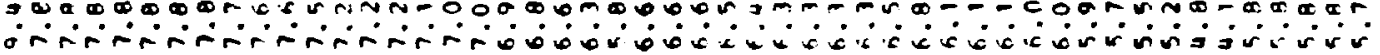

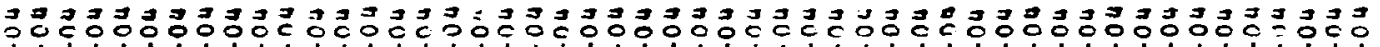

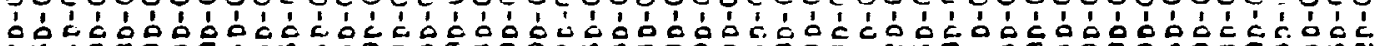

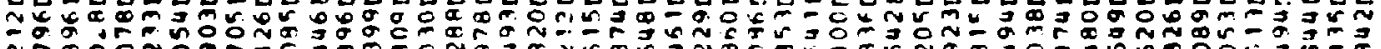
No 0 Conoor

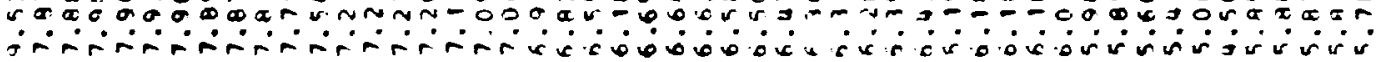

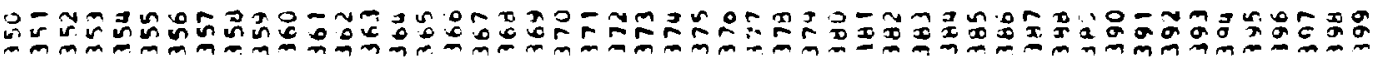




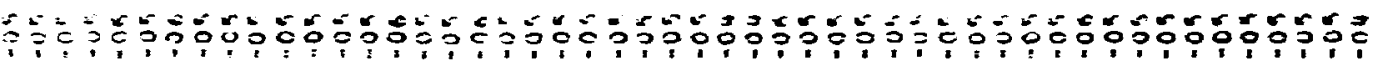

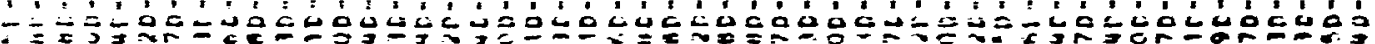

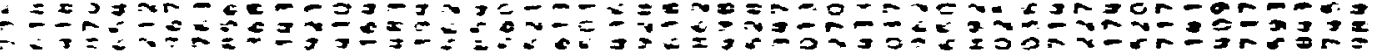

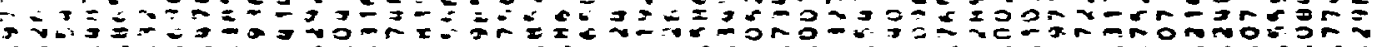
3 ;

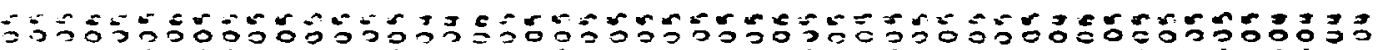

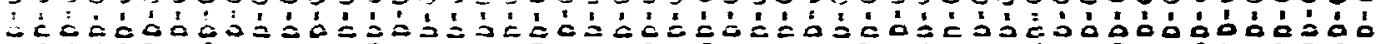

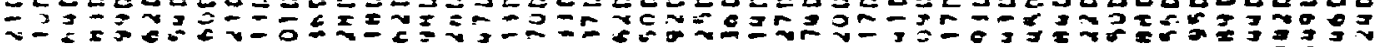

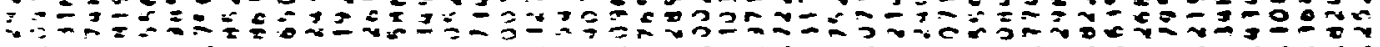

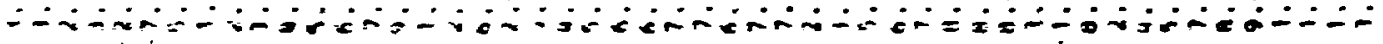

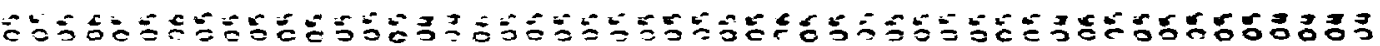

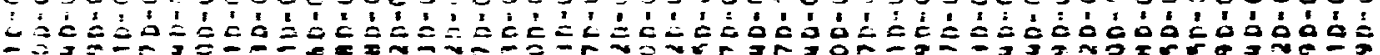

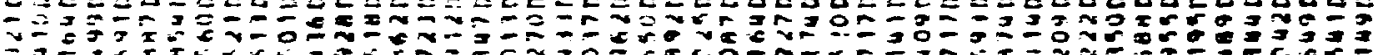

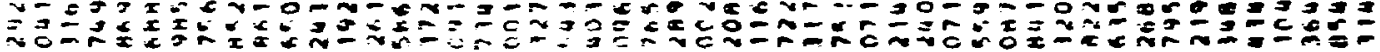

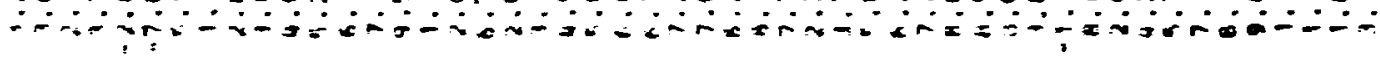

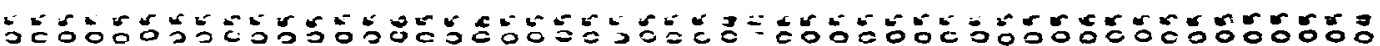

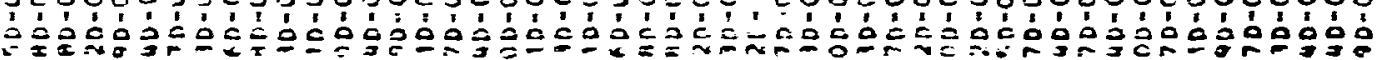

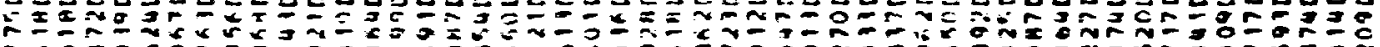

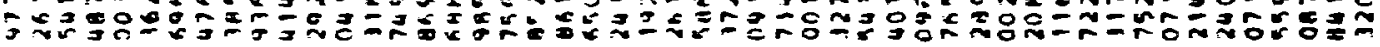

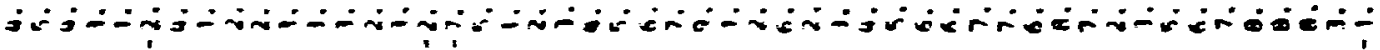

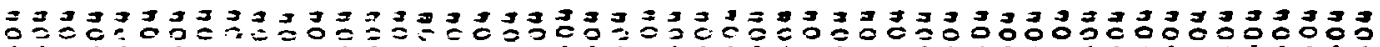

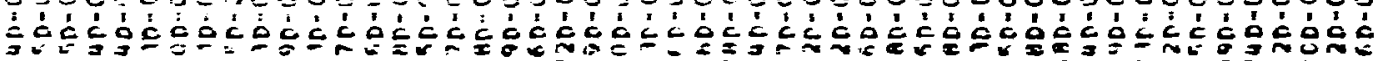

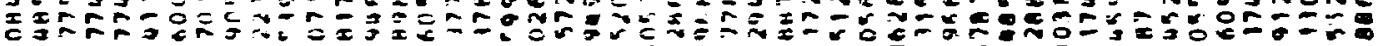
cos?

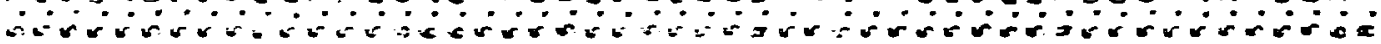

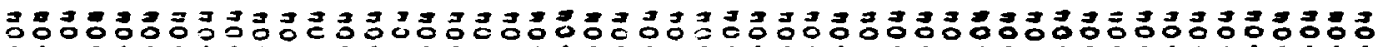
b́óódód

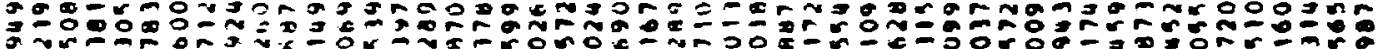

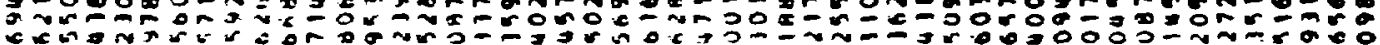

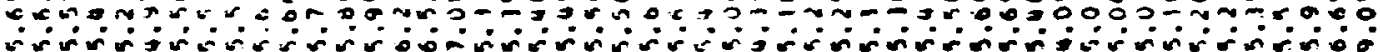

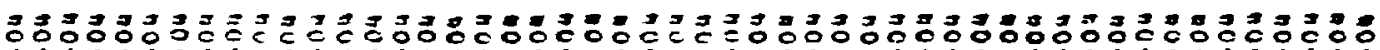

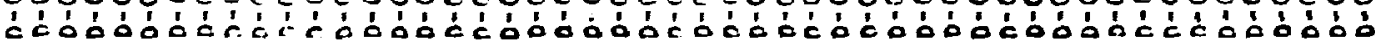

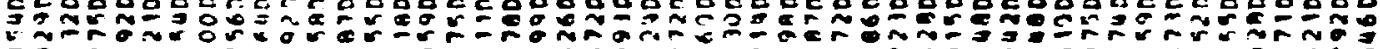

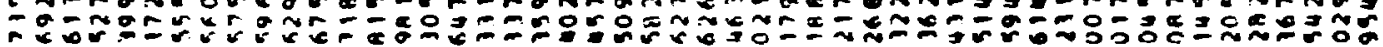

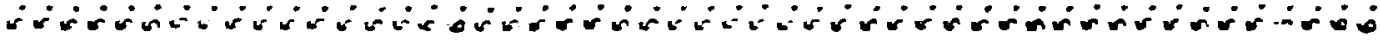

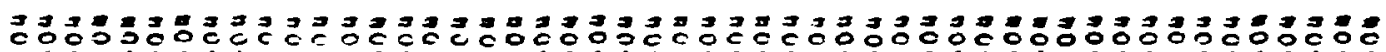

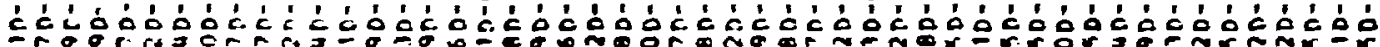

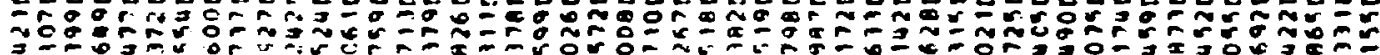

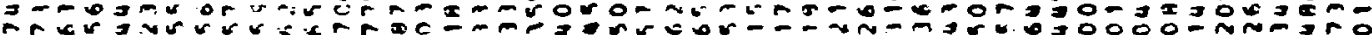

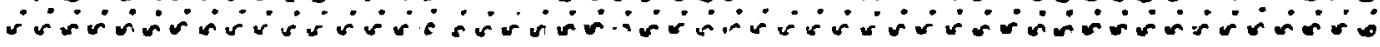

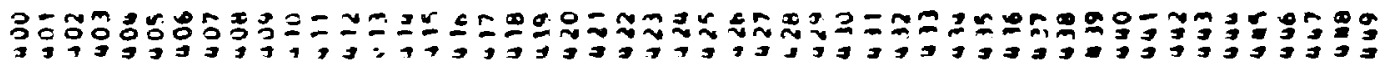




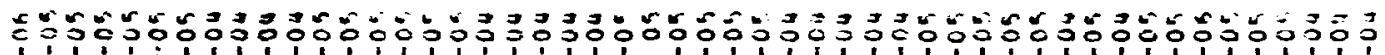

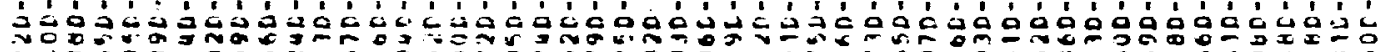

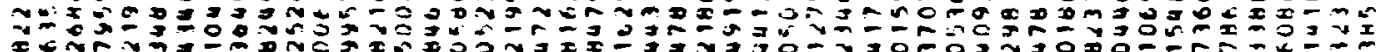

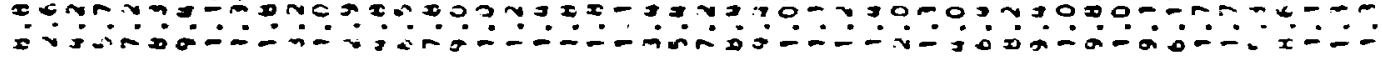

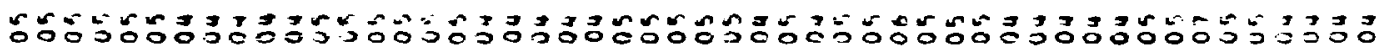

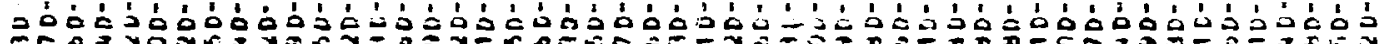

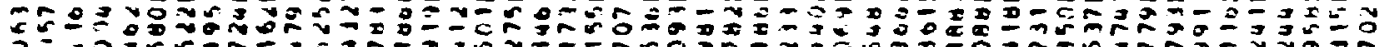

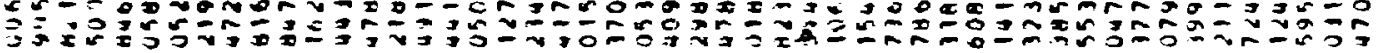
-

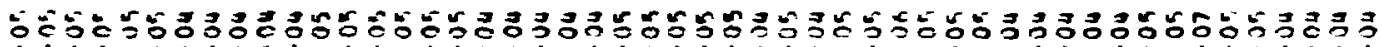

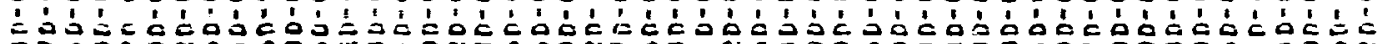

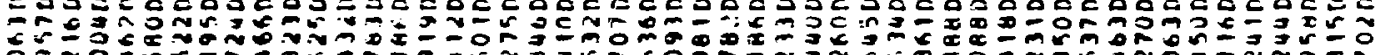

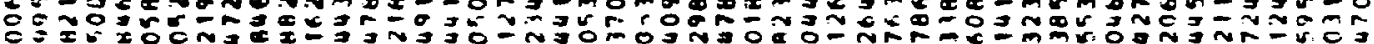

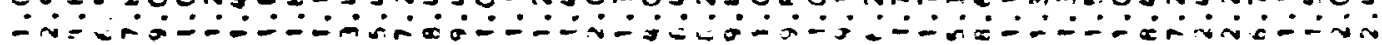

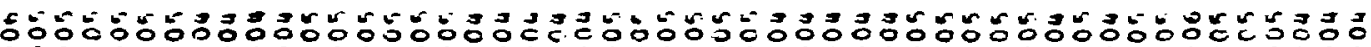

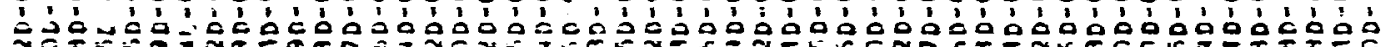

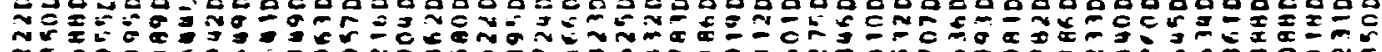

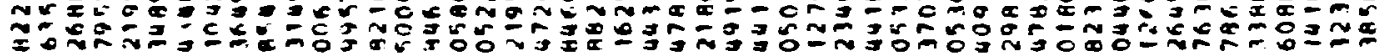

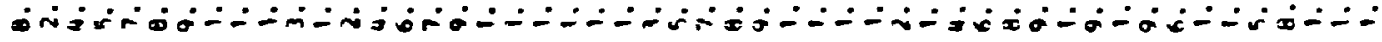

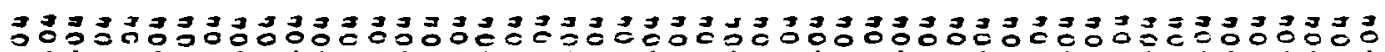

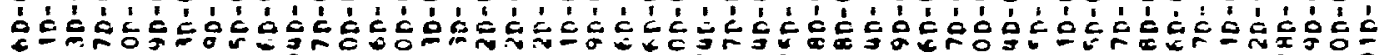

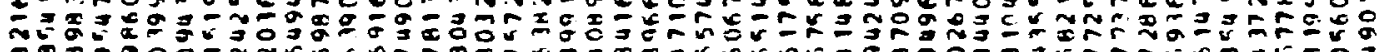

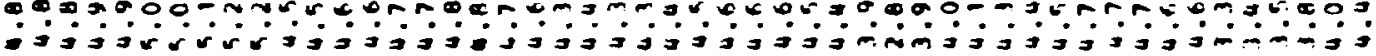

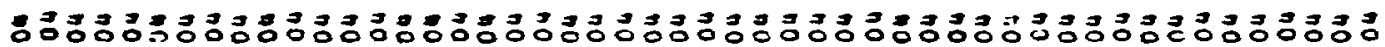

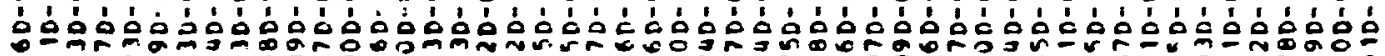

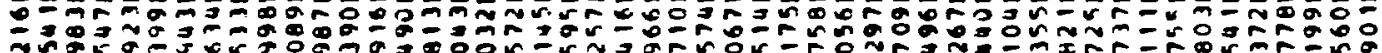
อง

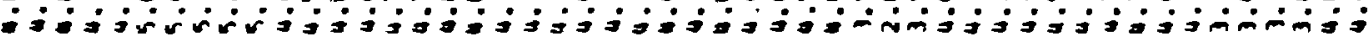

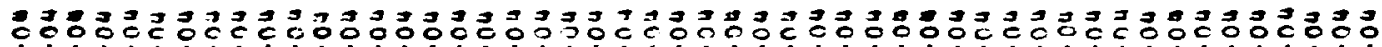

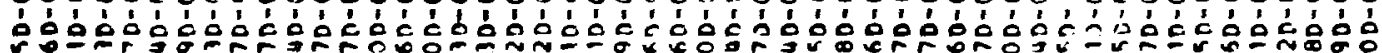

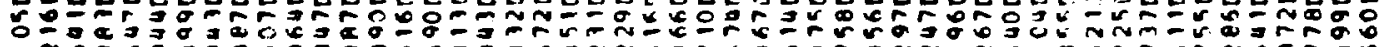

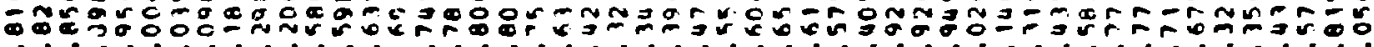

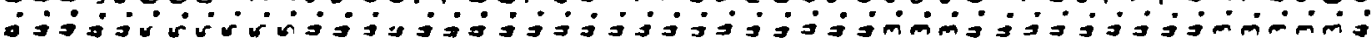

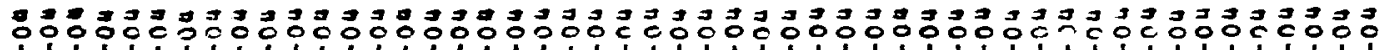
d́d

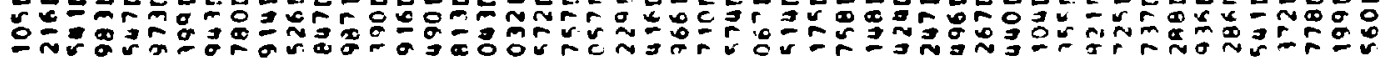

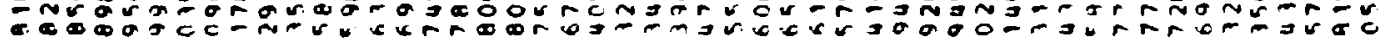
;

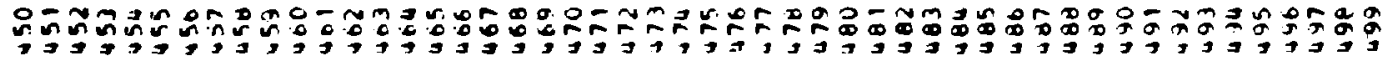




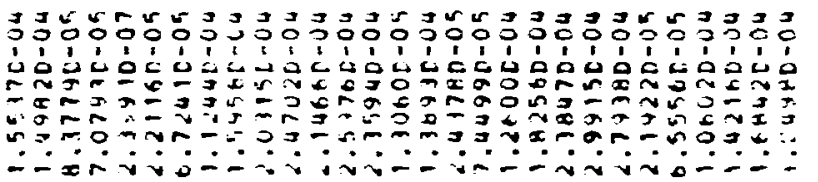

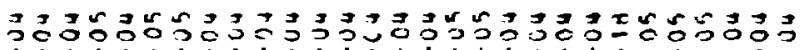

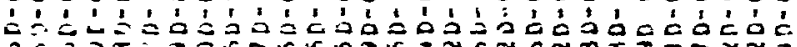

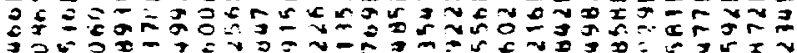

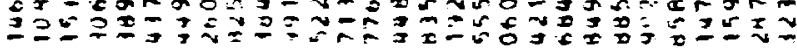

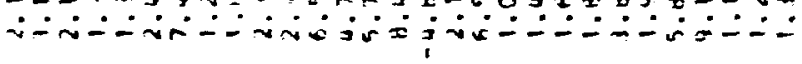

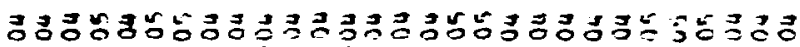

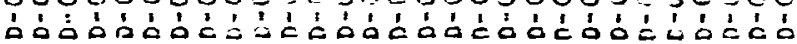

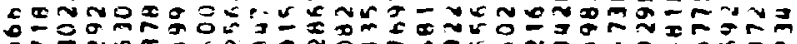

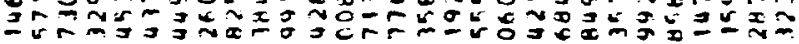

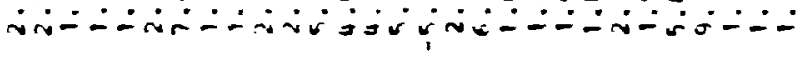

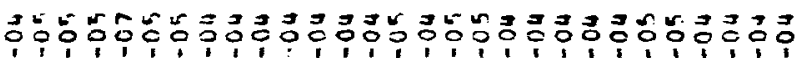

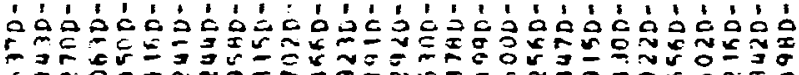

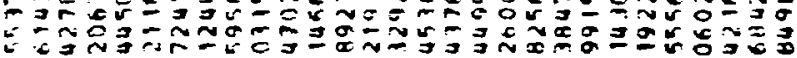

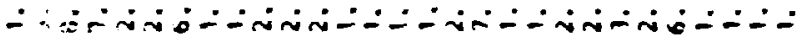

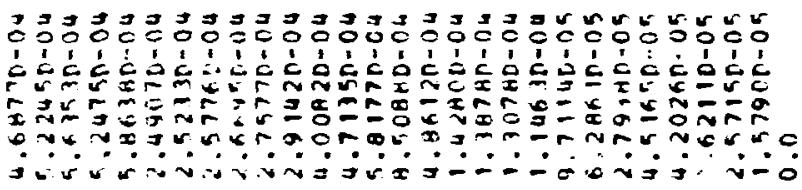

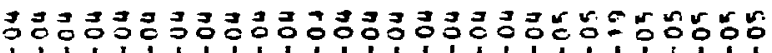

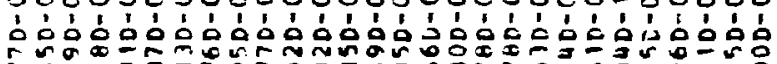

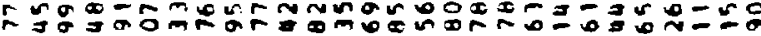
D in

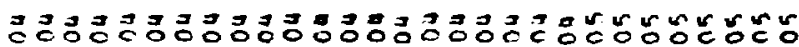

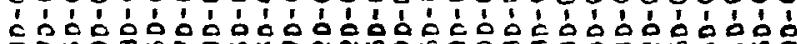

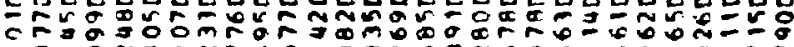

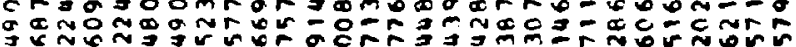

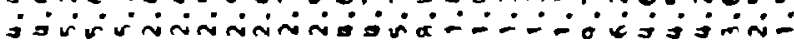

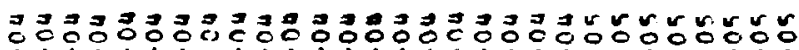

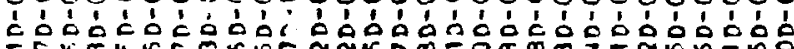

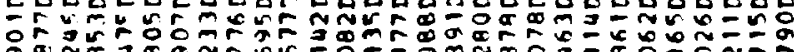
Tan

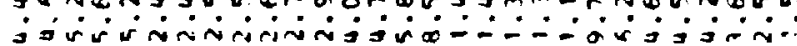

วอธิว 


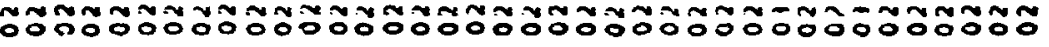

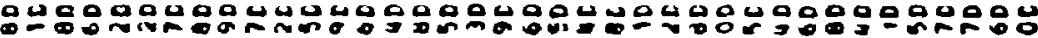

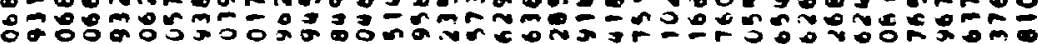

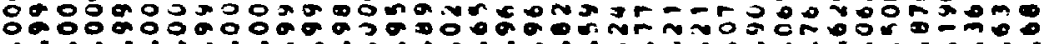

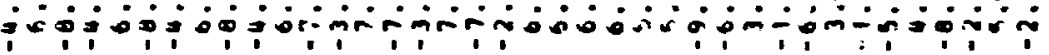

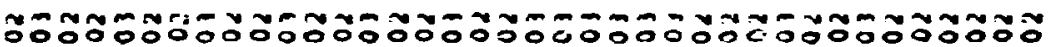

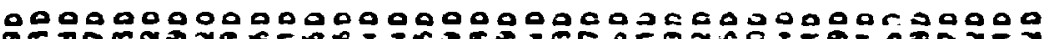

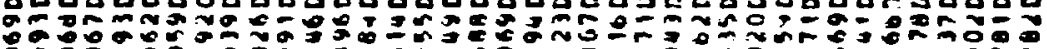

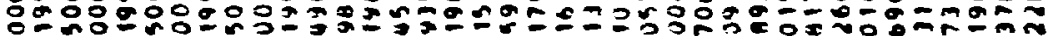

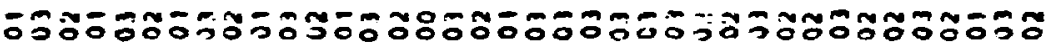

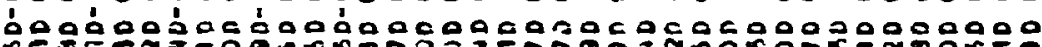

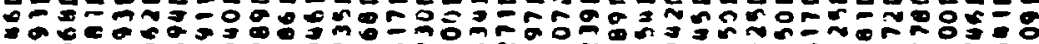

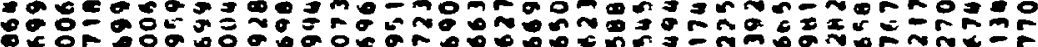

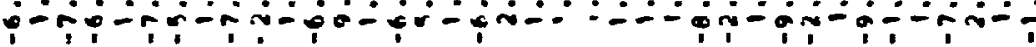

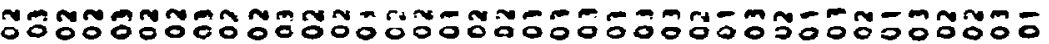

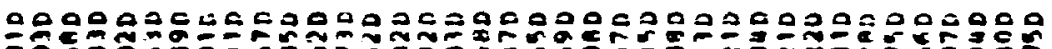

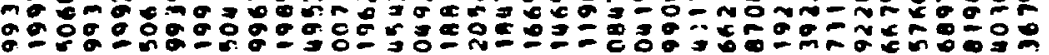

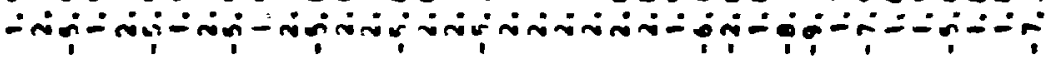

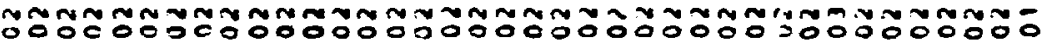

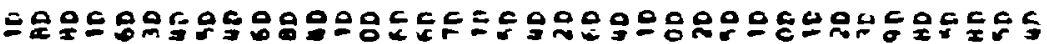

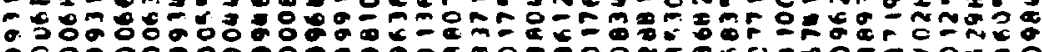

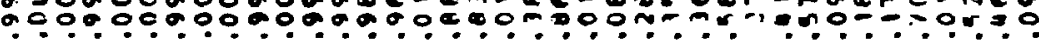

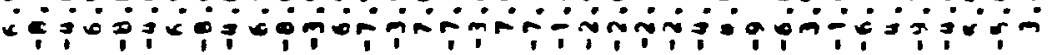

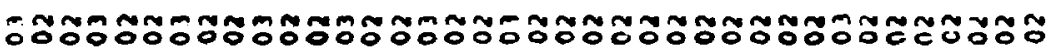

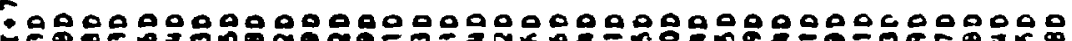

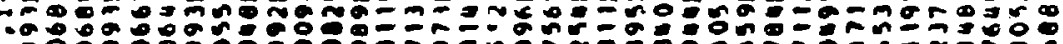

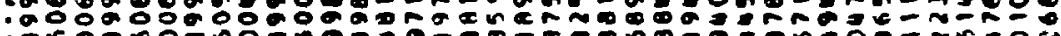

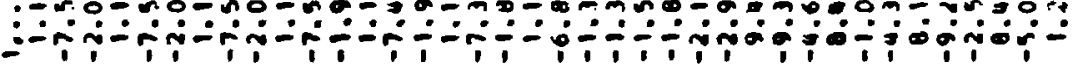
$\div$

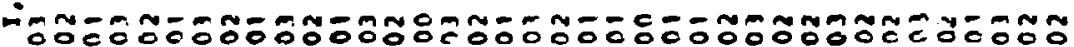

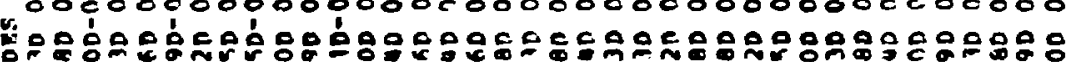

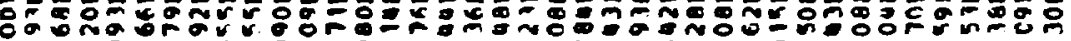

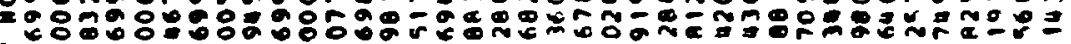
e-

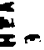

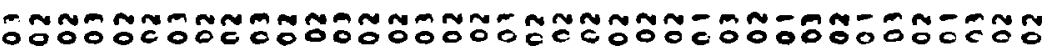

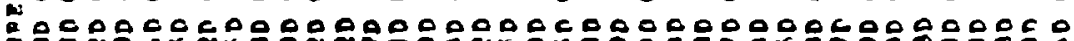

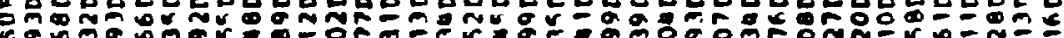

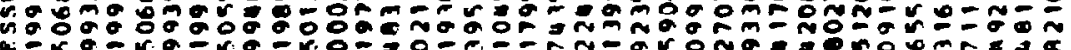

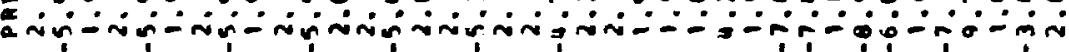

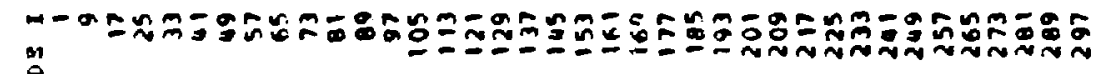
$\stackrel{1}{2}$ 


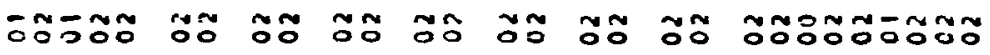

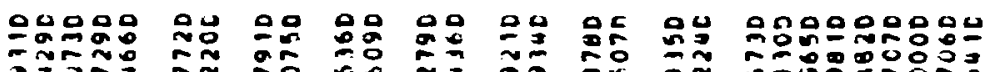

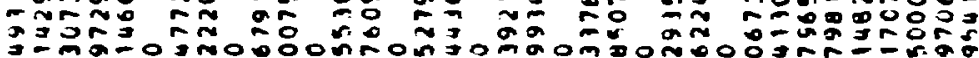

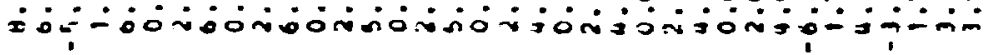

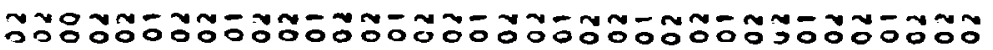

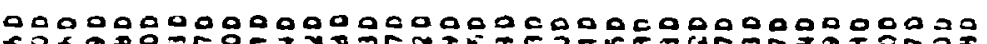

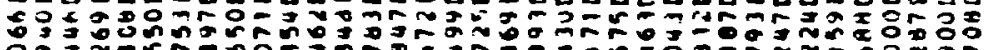

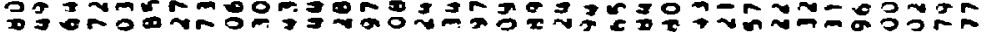

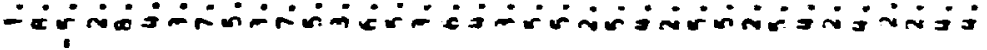

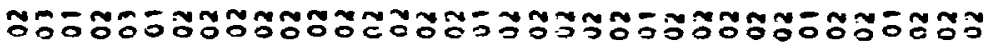

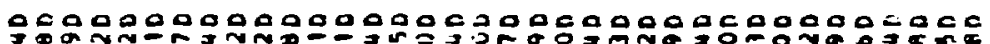

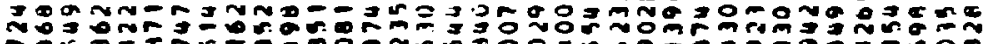

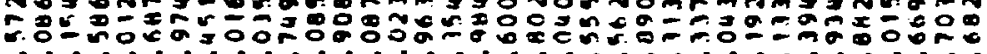

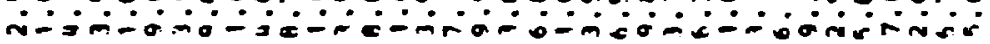

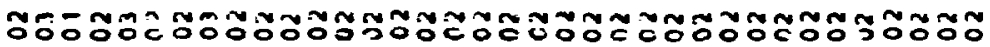

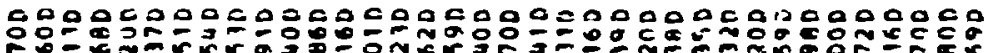

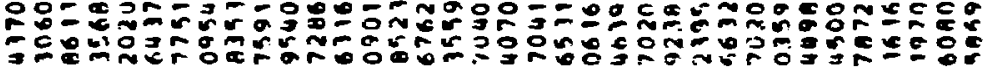

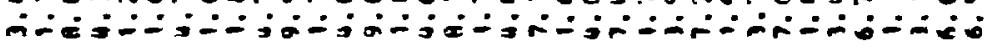

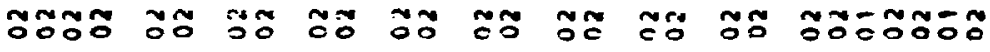

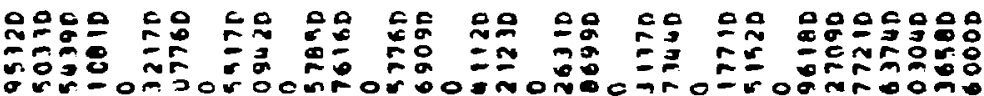

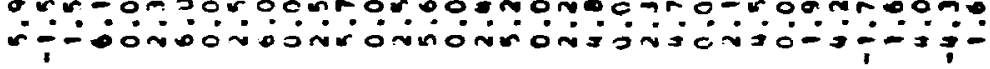

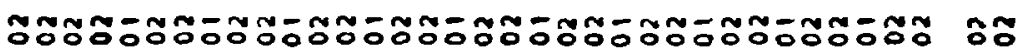

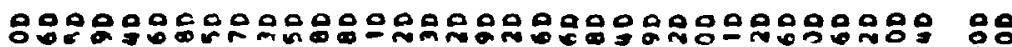

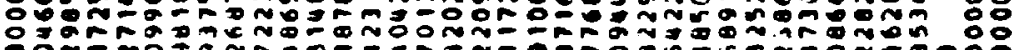

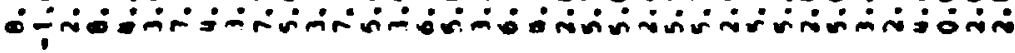

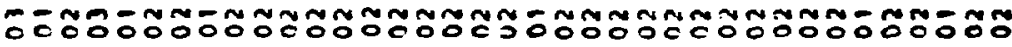

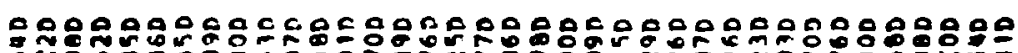

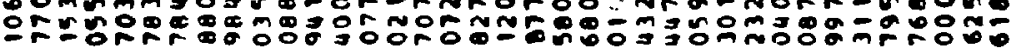

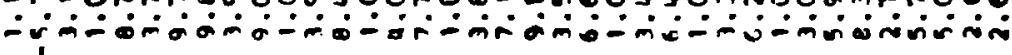

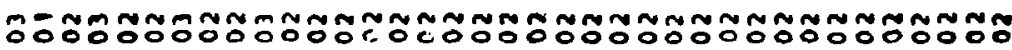

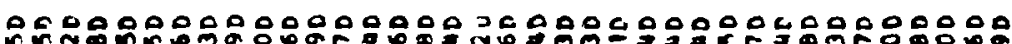
К

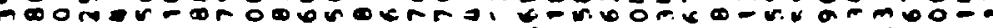

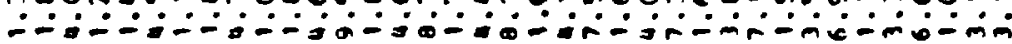

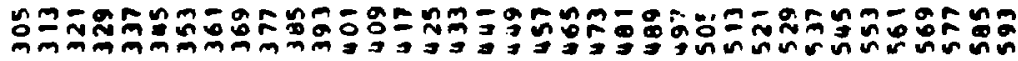




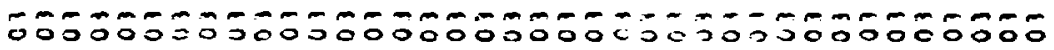

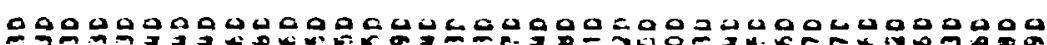

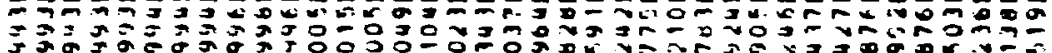

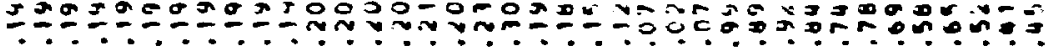

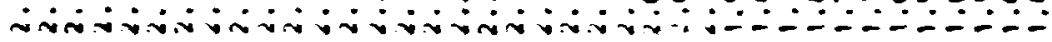

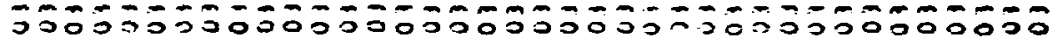

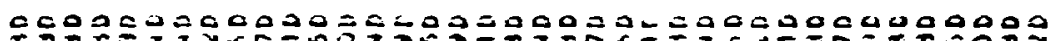

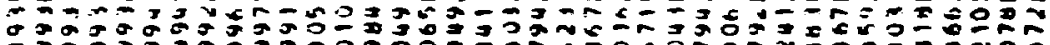

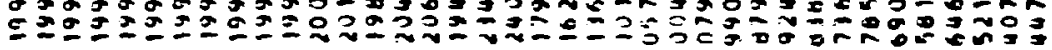

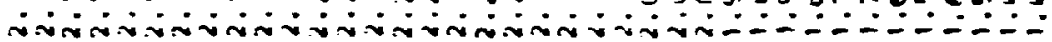

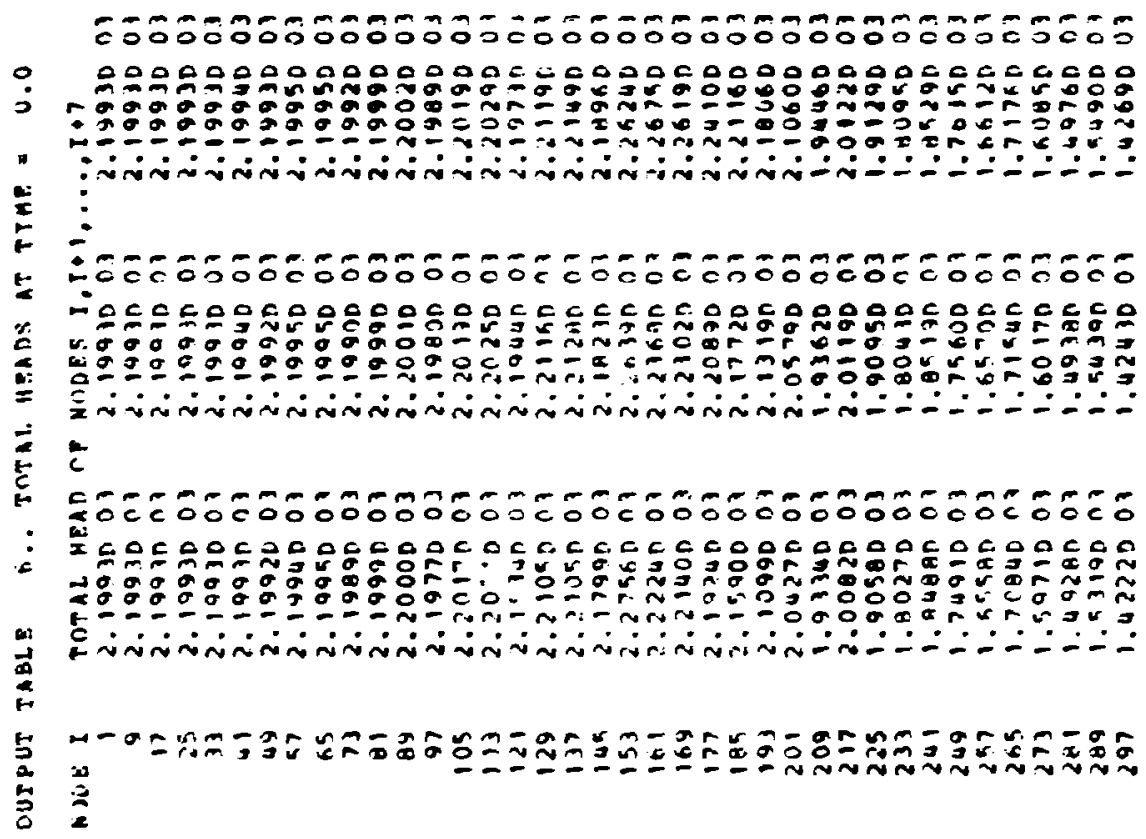

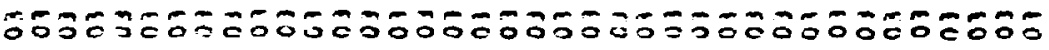

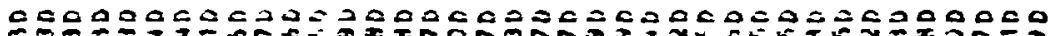

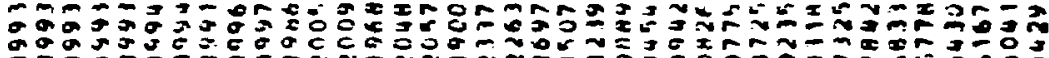

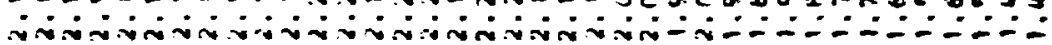

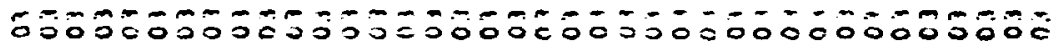

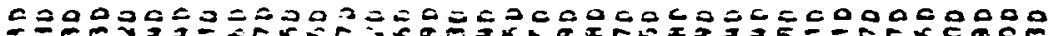

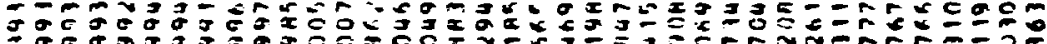

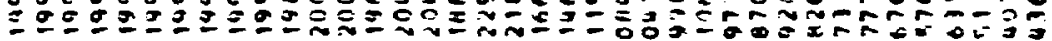

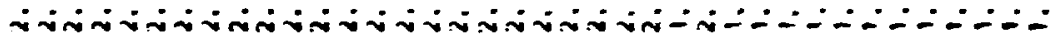

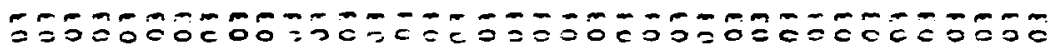

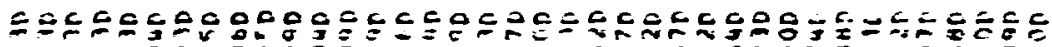

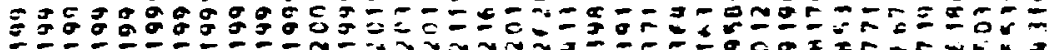

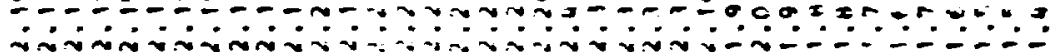

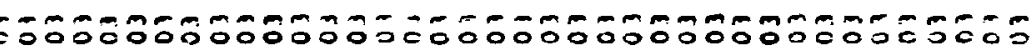

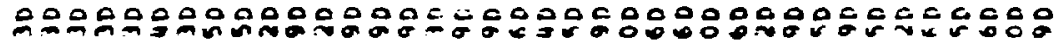

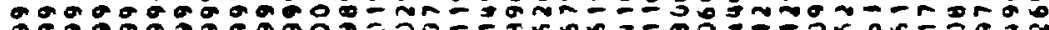

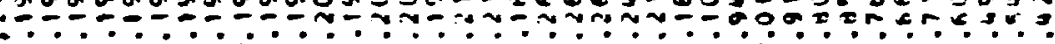

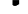

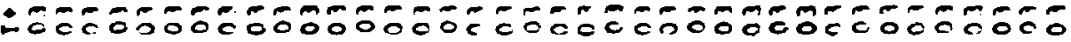

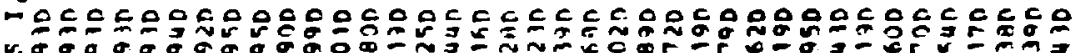

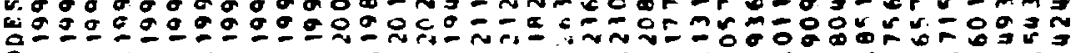

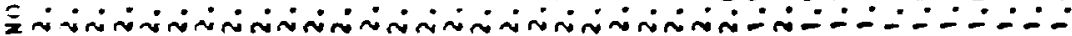

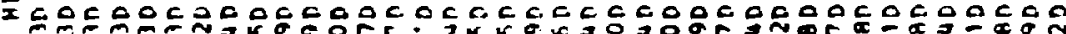

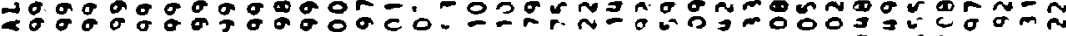

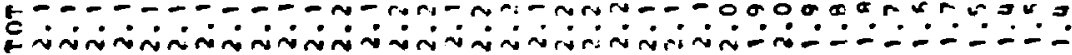

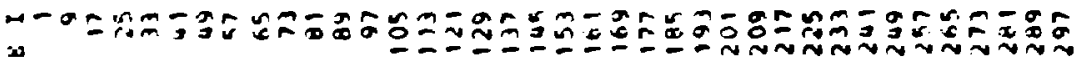
2 


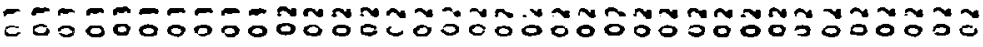

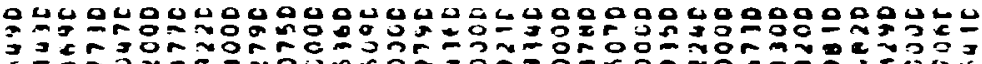

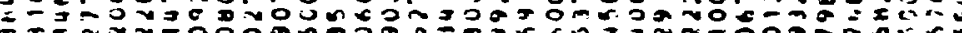

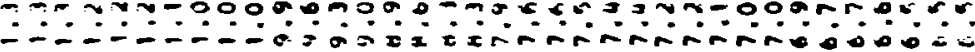

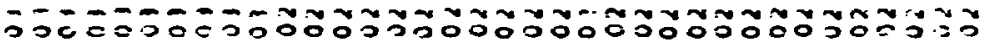

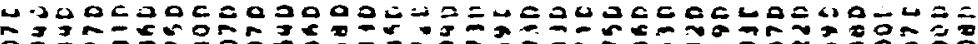

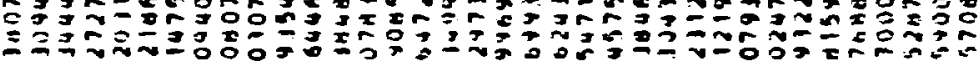

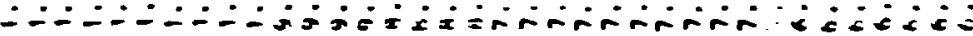

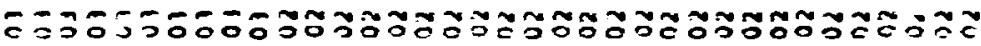

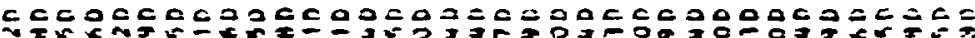

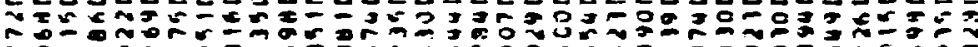

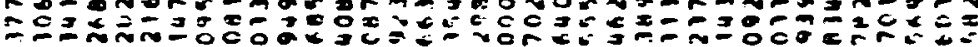

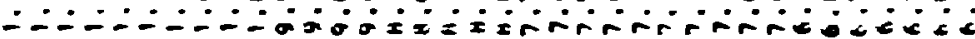

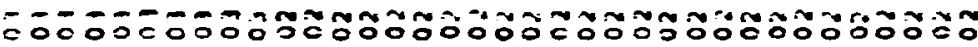

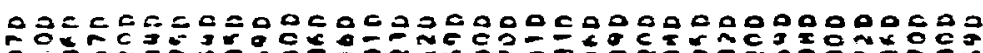

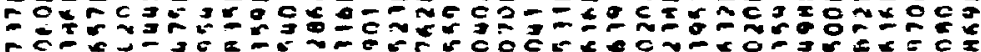

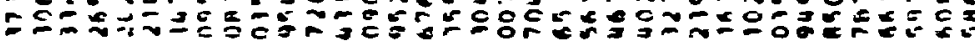

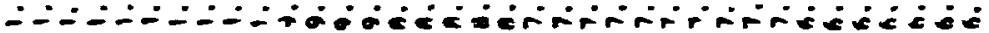

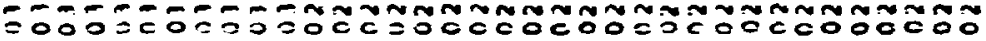

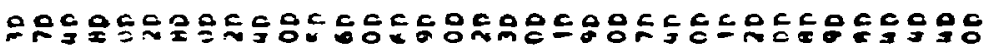

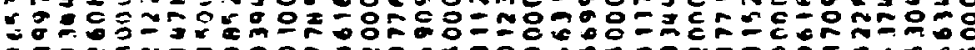
T-r.

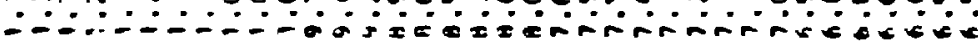

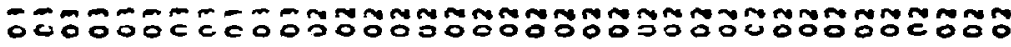
DOogacodco00000000000000c00000000000

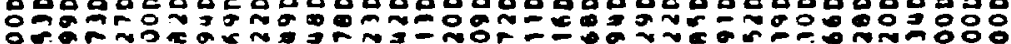

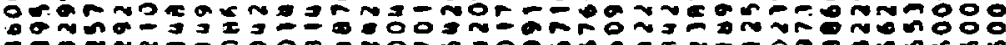
Z:-

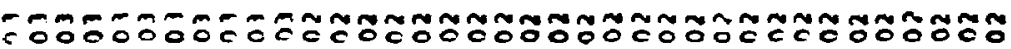

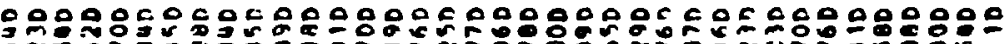
슴ำ

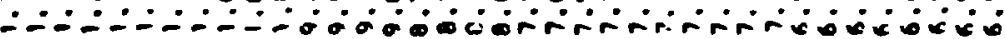

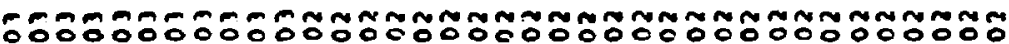

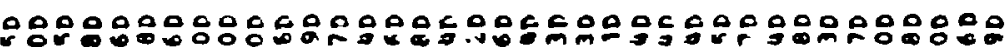

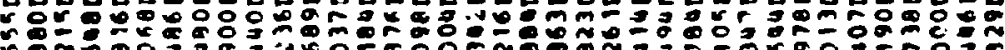
rmm

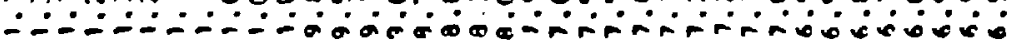

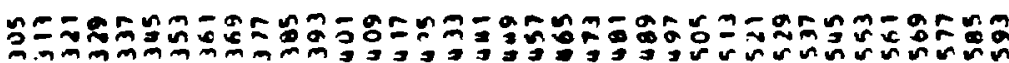




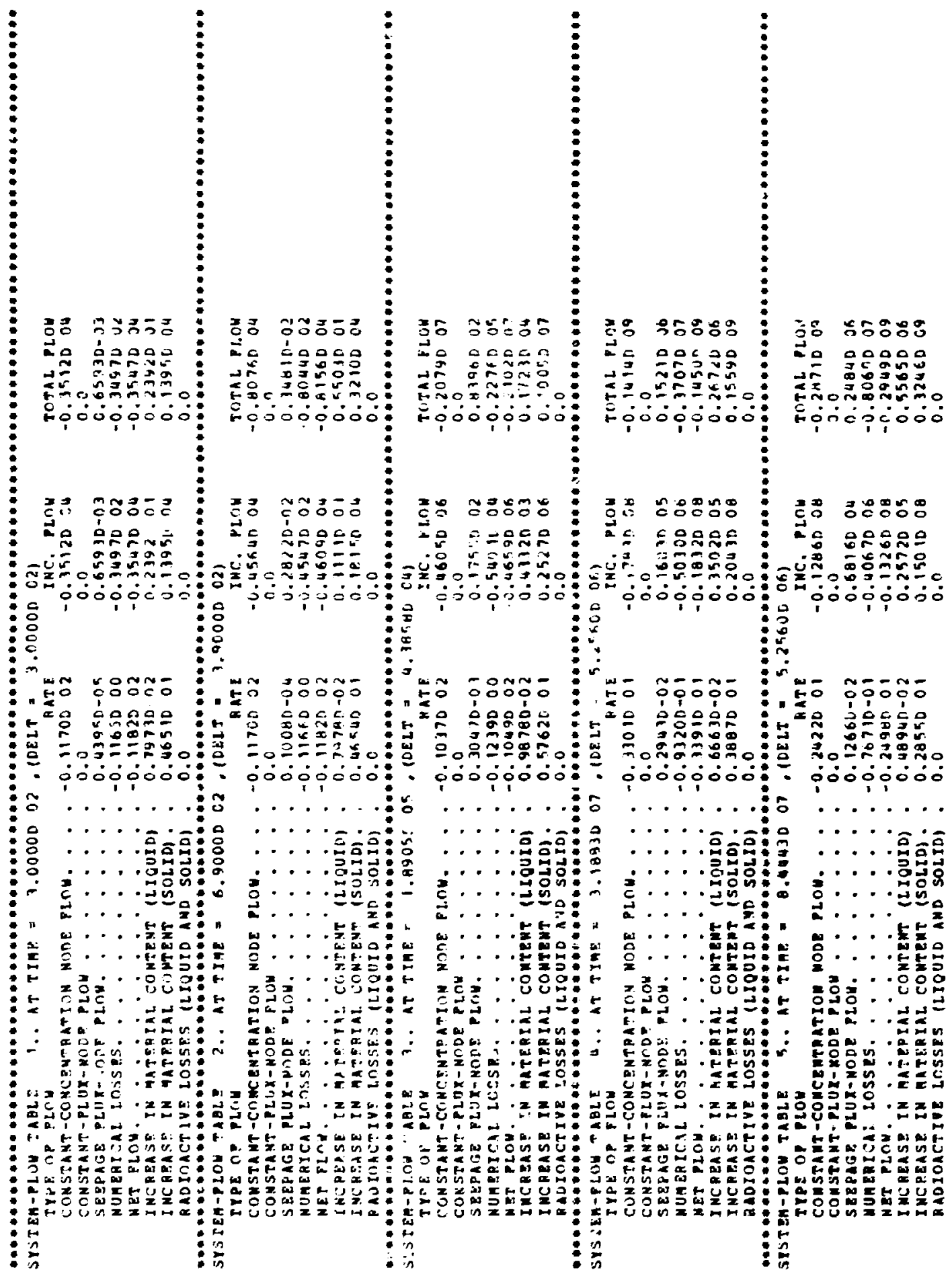




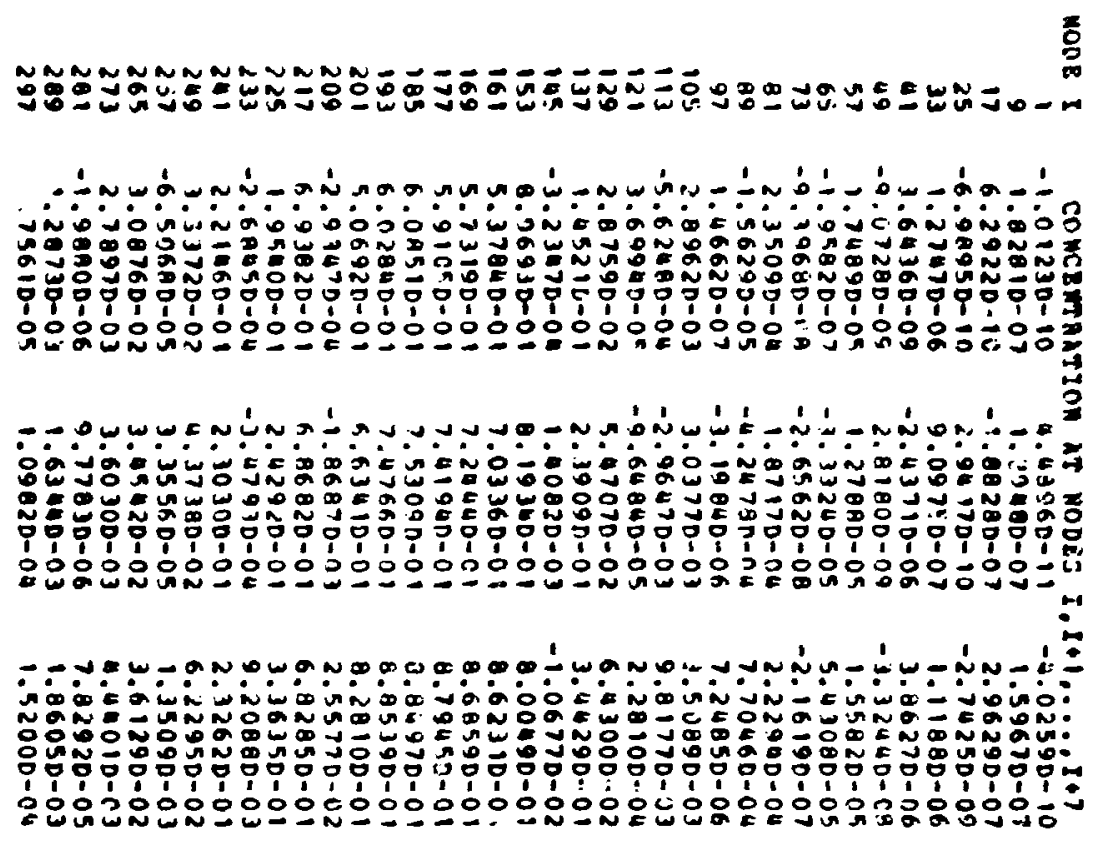

$\sum_{\substack{2 \\ \vdots}}^{2}$

น N N

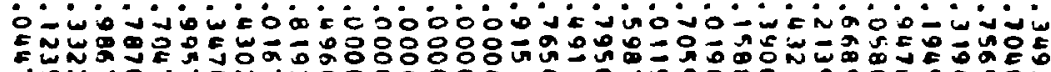

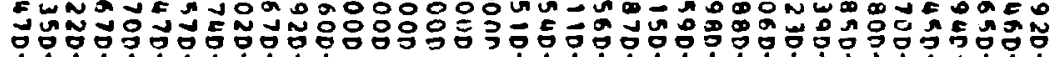

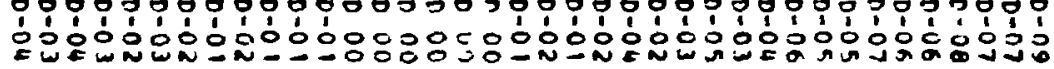

س

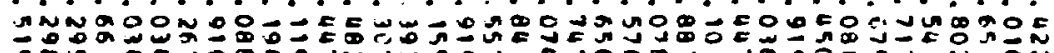

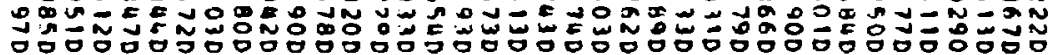

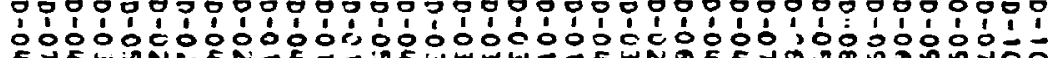

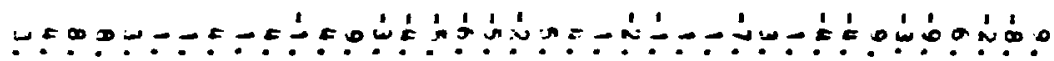
更o

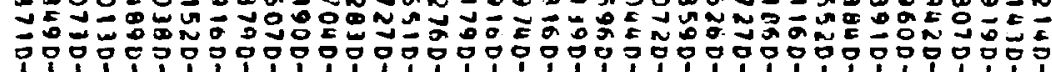

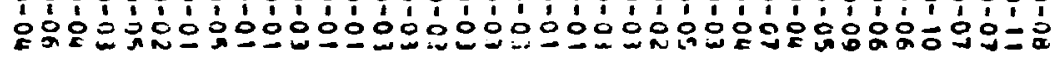

"

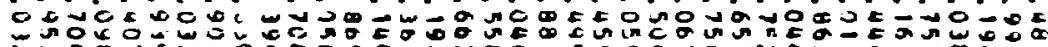

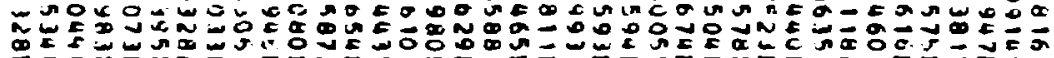

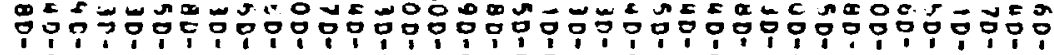

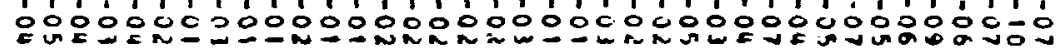

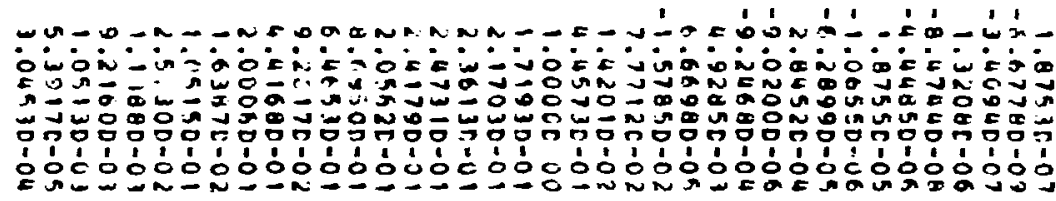




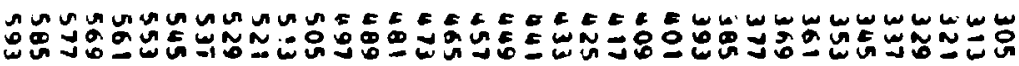

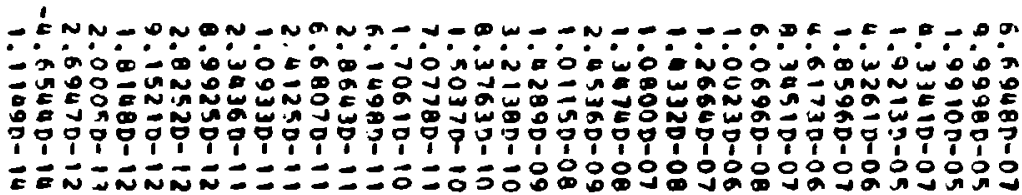

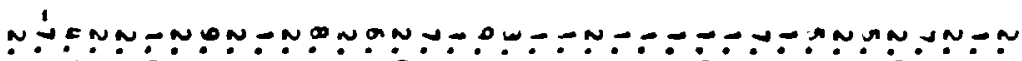

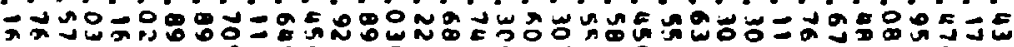

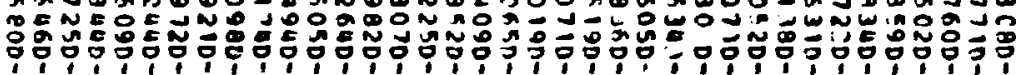

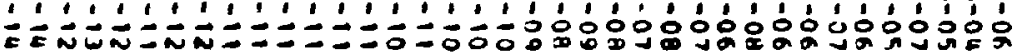

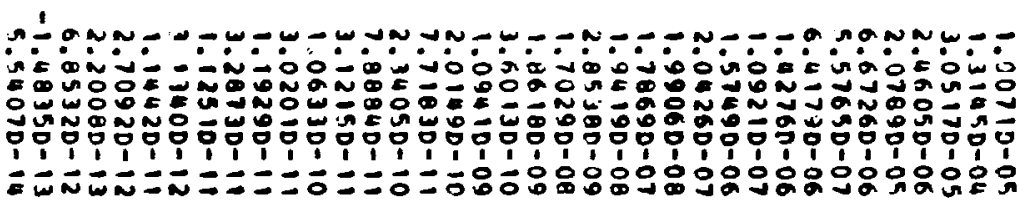

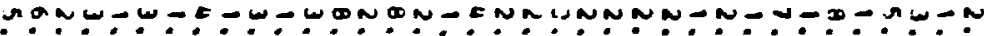
añ

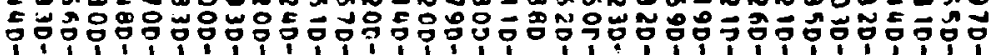

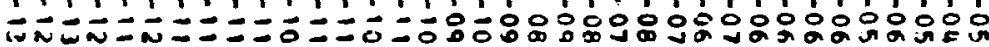

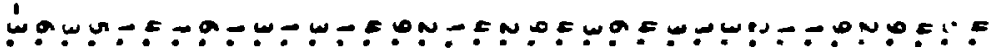
OO

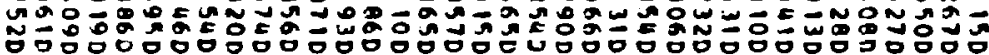

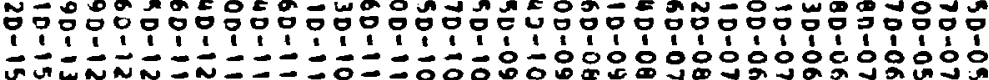

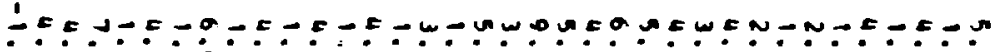

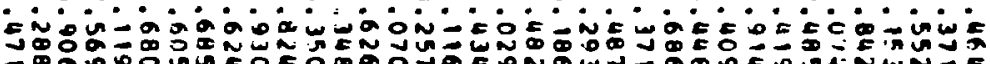

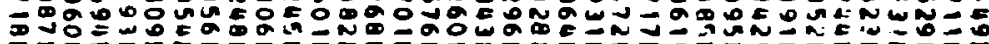

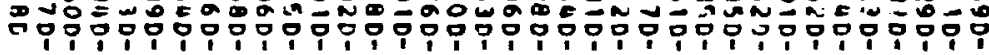

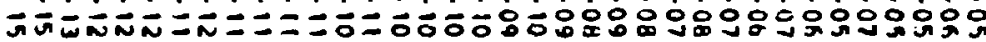

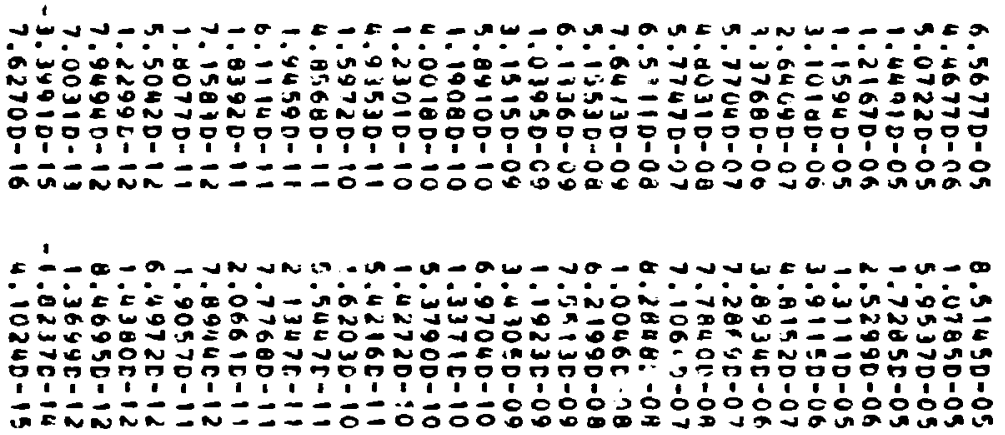




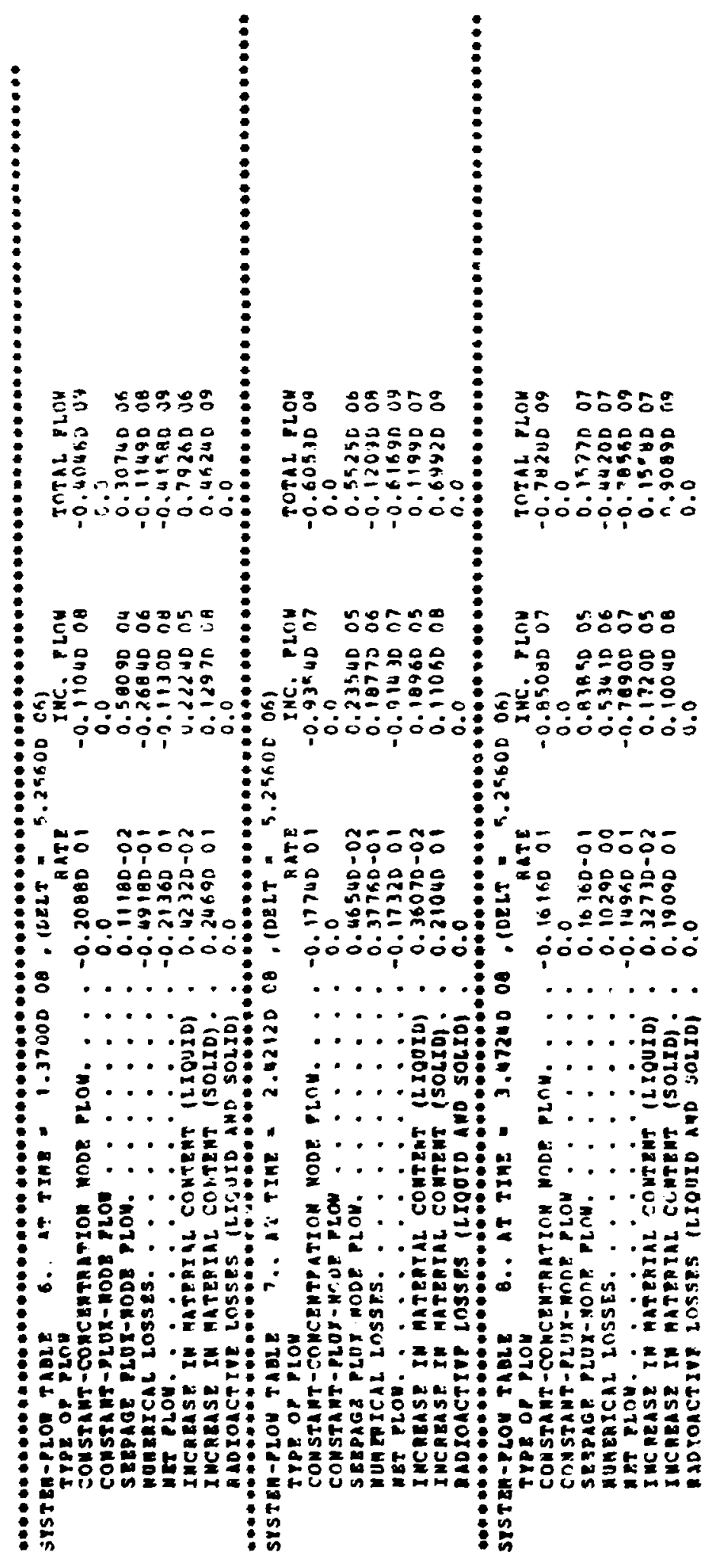




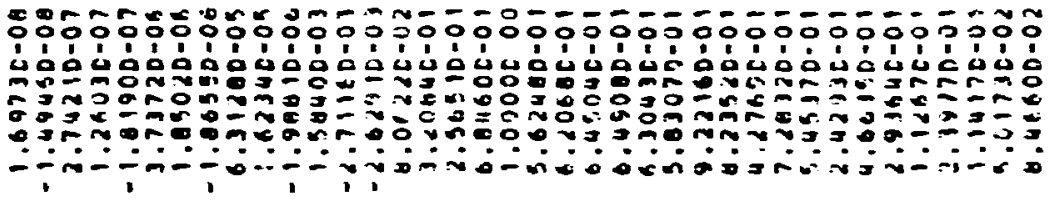

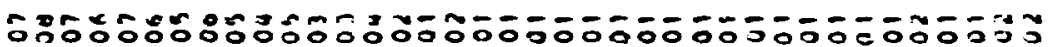

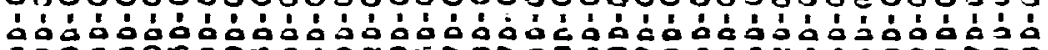

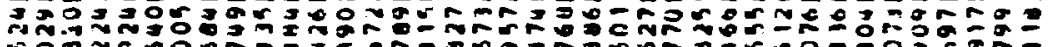

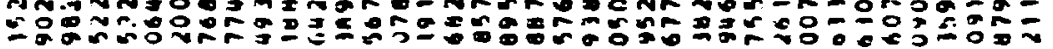

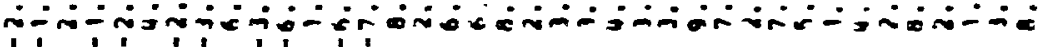

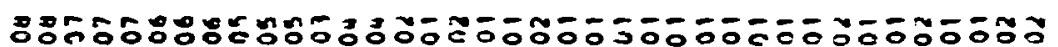

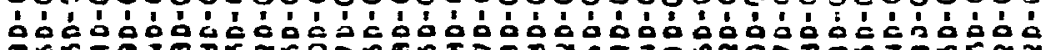

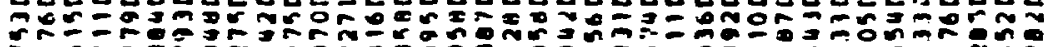

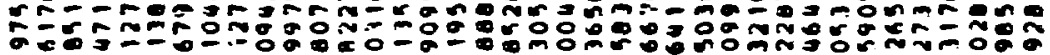

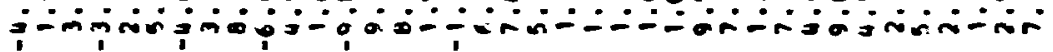

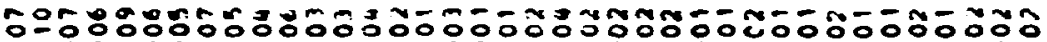

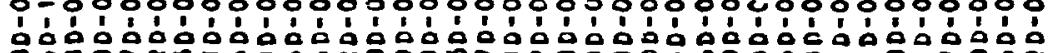

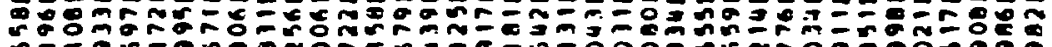

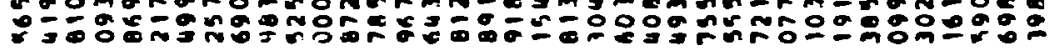

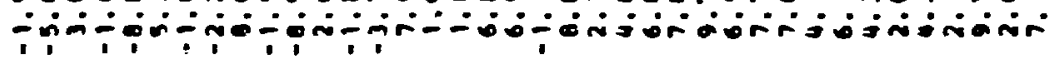

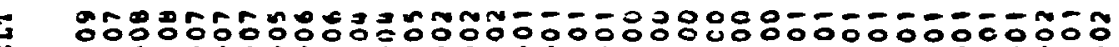

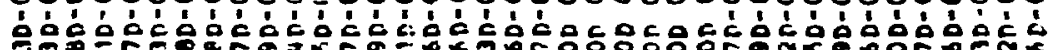

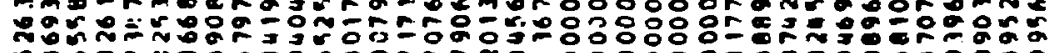
n.

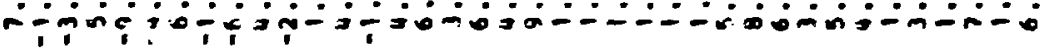

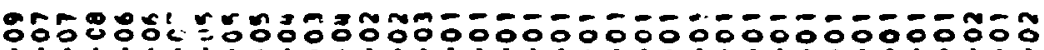
૧

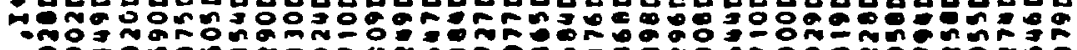

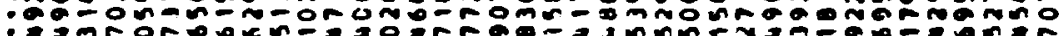

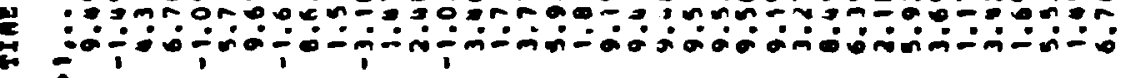

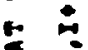

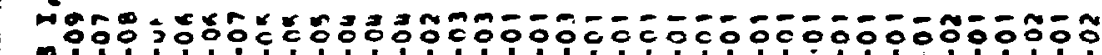
o

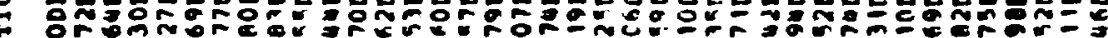

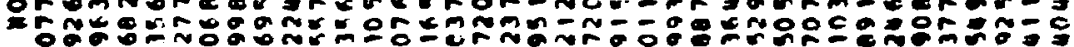

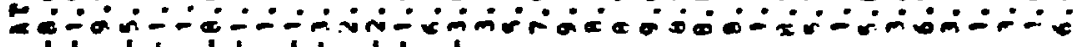
要

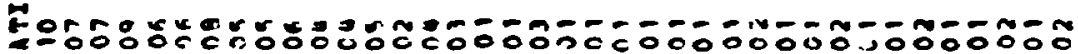

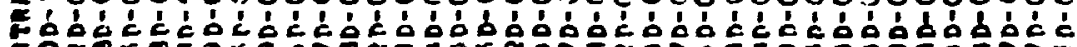

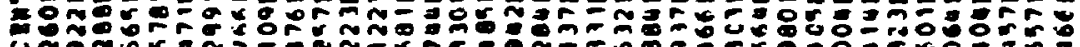
Uñ

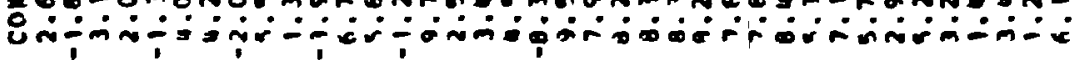

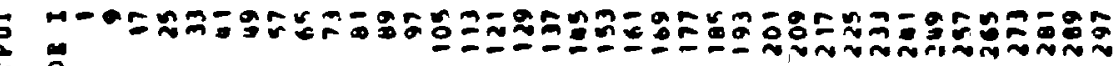
官 


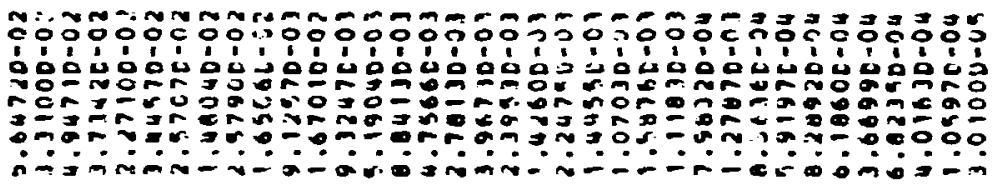

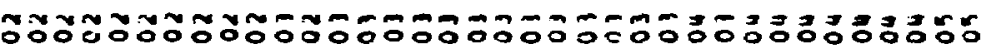

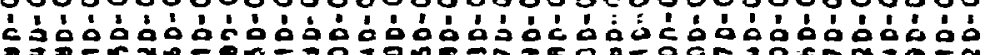

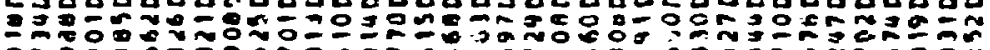

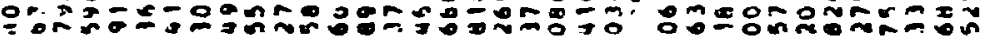

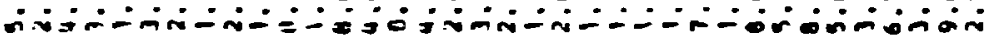

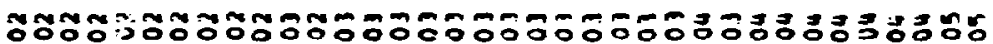

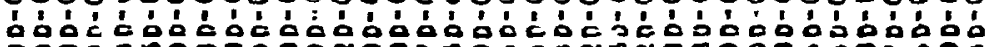

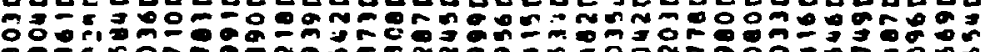

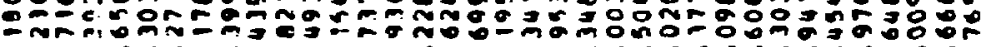

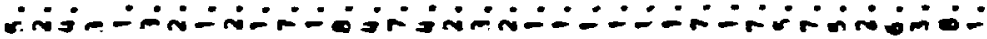

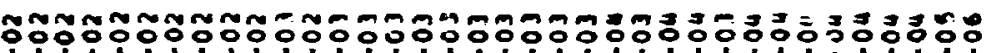

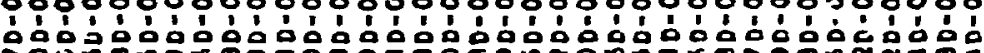
웅

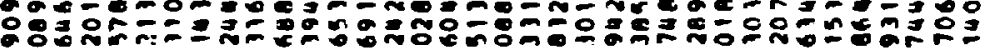

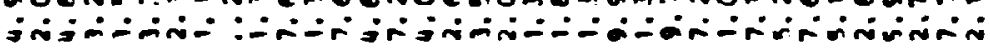

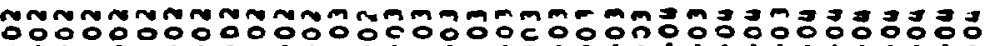

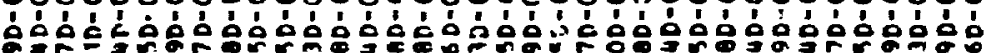

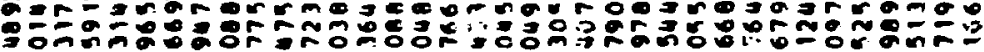
oñ

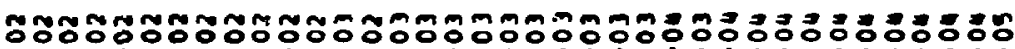

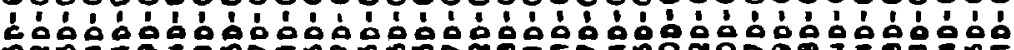

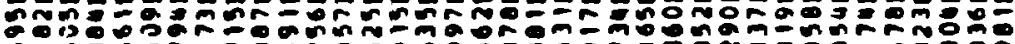
ñe

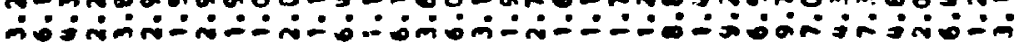

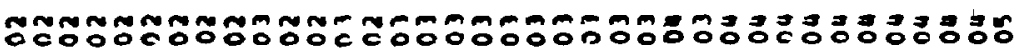

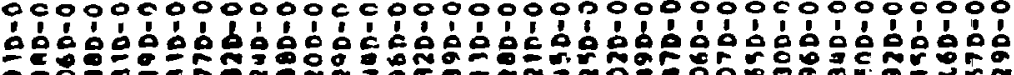
- -omm-chen

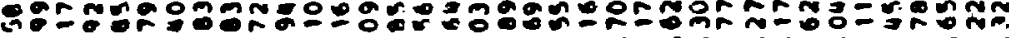

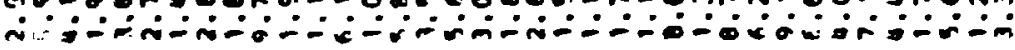

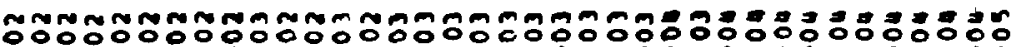

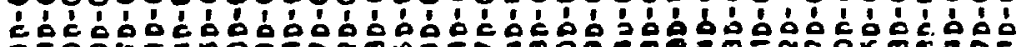

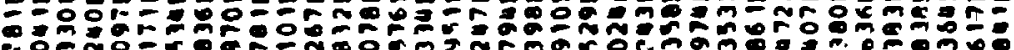

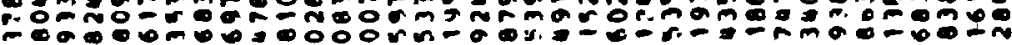
rún-

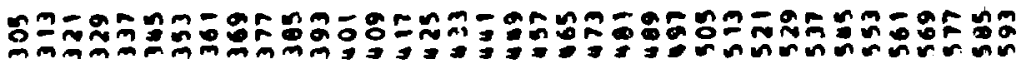




\begin{tabular}{|c|c|c|c|c|c|}
\hline 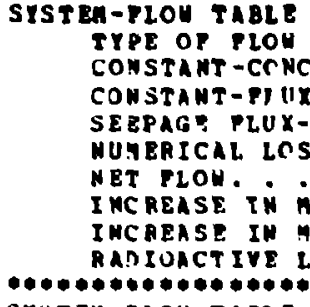 & 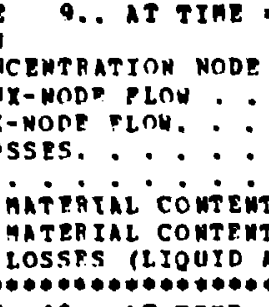 & 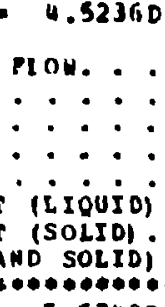 & 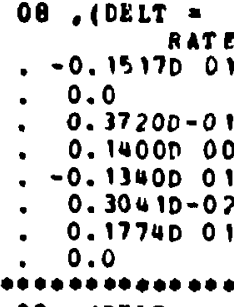 & 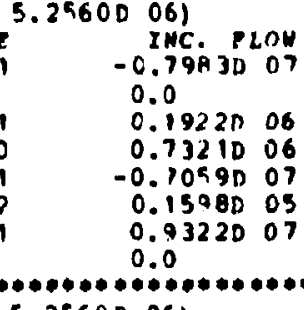 & $\begin{array}{rl}\text { TOTAL } & 104 \\
-0.011710 & 09 \\
0.0 & \\
0.43140 & 07 \\
0.05430 & 07 \\
-0.93420 & 09 \\
0.18890 & 07 \\
0.11020 & 10 \\
0.0 & \end{array}$ \\
\hline 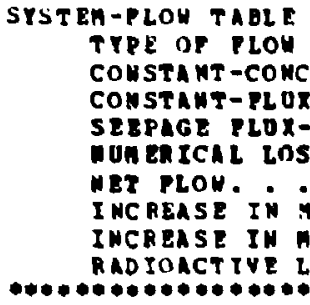 & 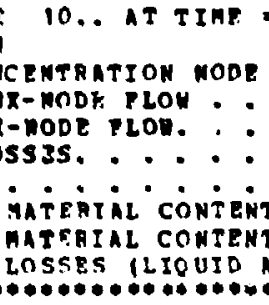 & 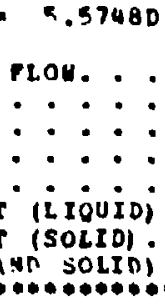 & 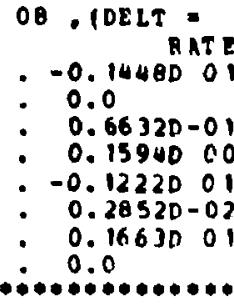 & 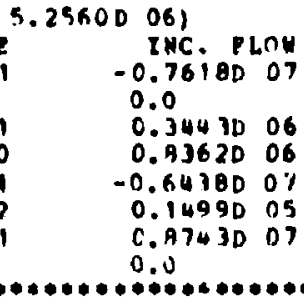 & $\begin{array}{l}\text { TCTAL PLOH } \\
-0.1103010 \\
0.0 \\
0.9684007 \\
0.2439008 \\
-0.1069010 \\
0.2199007 \\
0.1282010 \\
0.0\end{array}$ \\
\hline 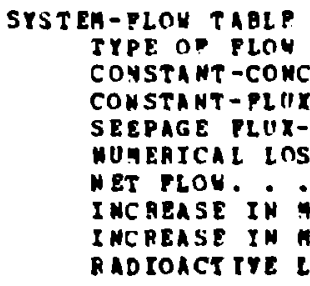 & 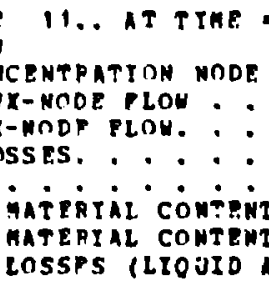 & 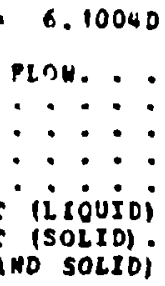 & 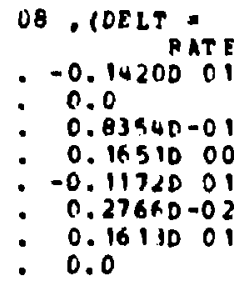 & 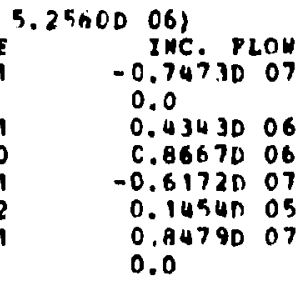 & $\begin{array}{ll}\text { THTAL PLON } \\
-0.11780 & 10 \\
0.0 & \\
0.13620 & 09 \\
0.13930 & 08 \\
-0.11320 & 10 \\
0.23490 & 07 \\
0.13680 & 10 \\
0.0 & \end{array}$ \\
\hline
\end{tabular}




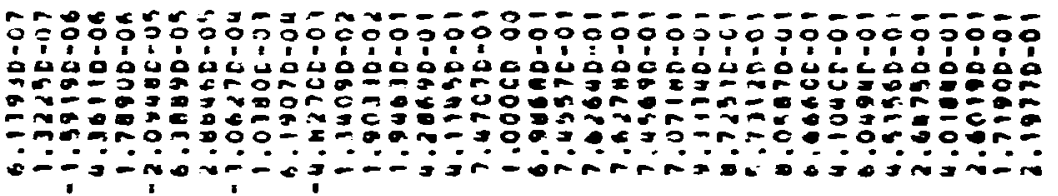

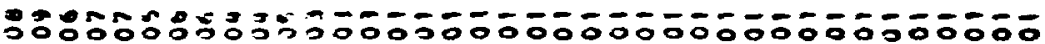

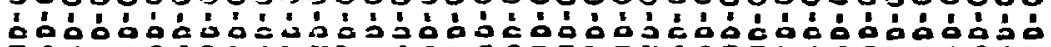

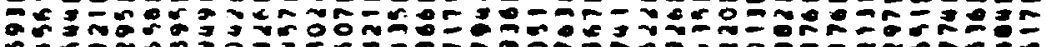

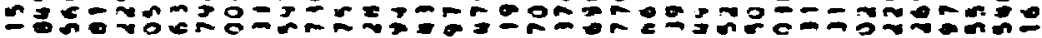

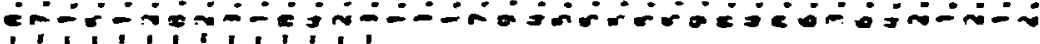

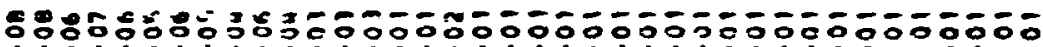

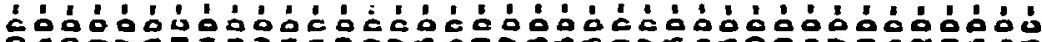

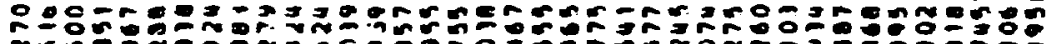

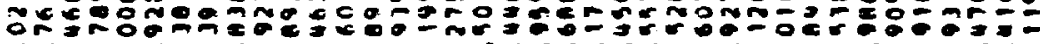

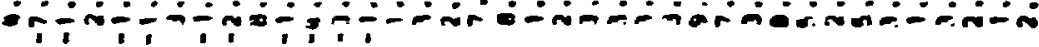

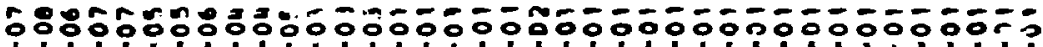

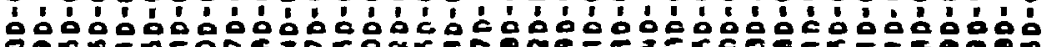

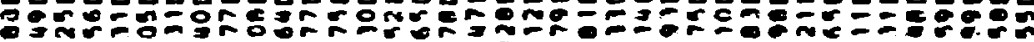

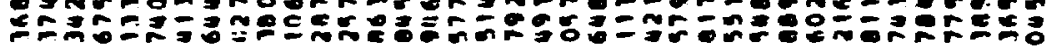

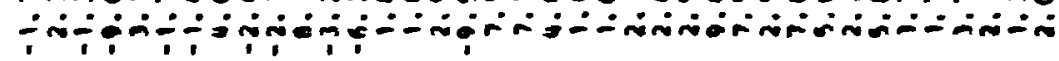

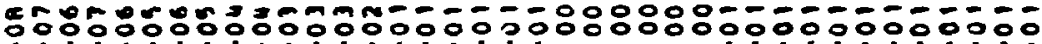

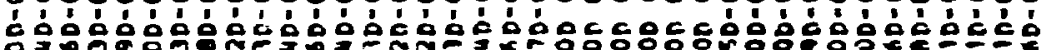

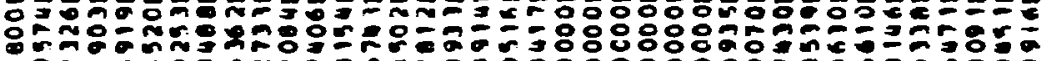

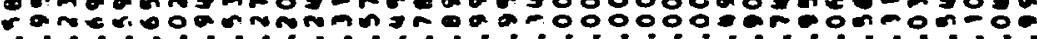

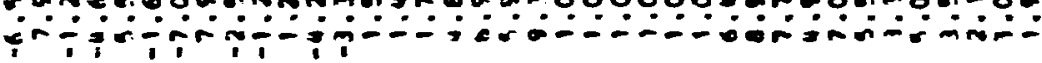

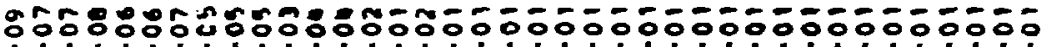

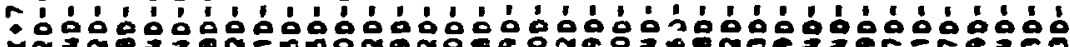

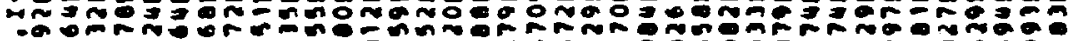

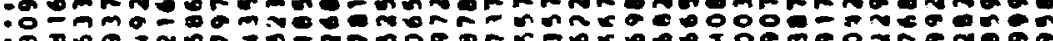

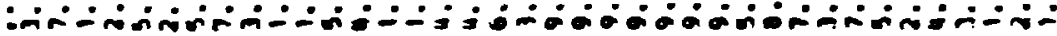
$\dot{*}$

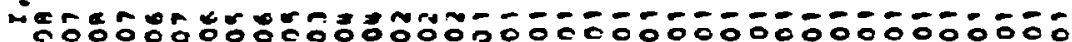

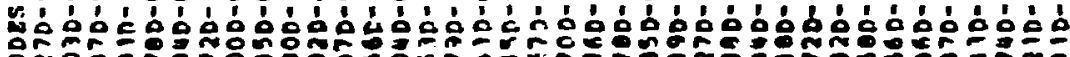

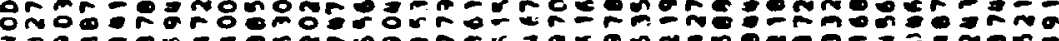

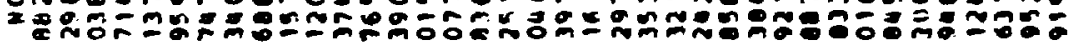

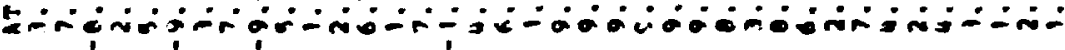
c

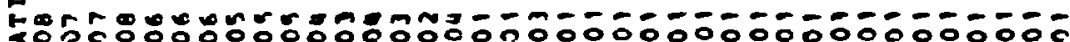

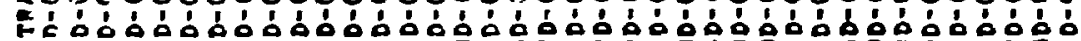

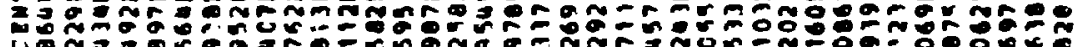

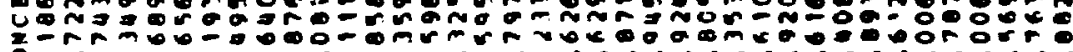

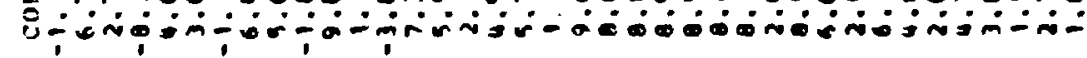

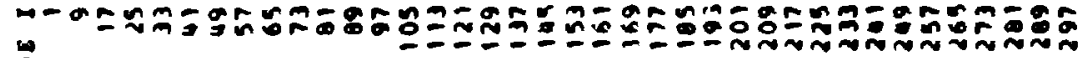
$\stackrel{\leftrightarrow}{\circ}$ 


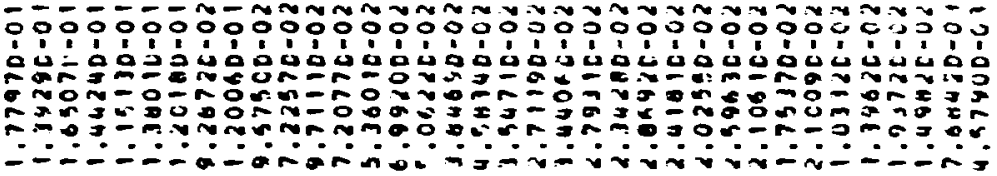

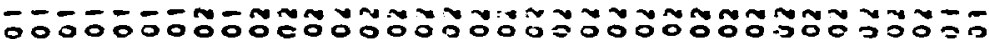

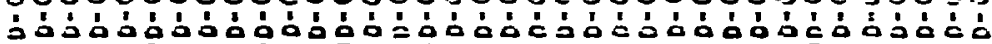

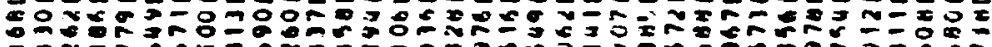

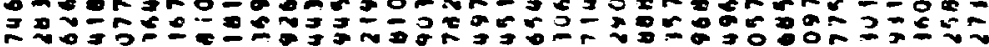

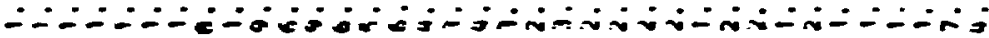

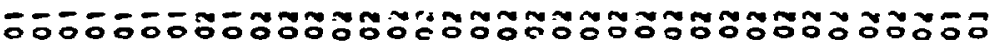

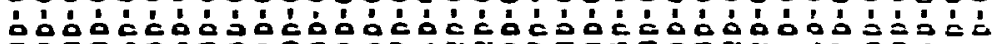

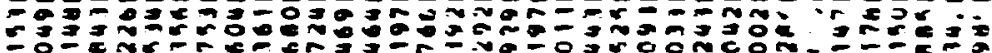

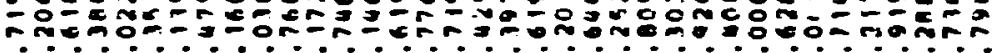

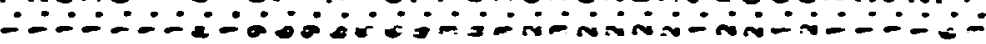

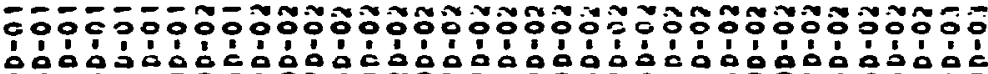

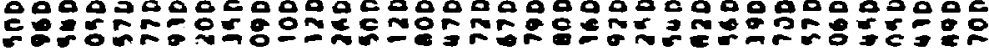

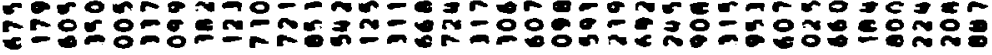

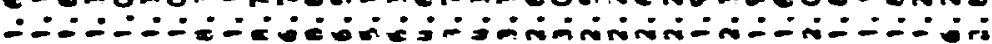

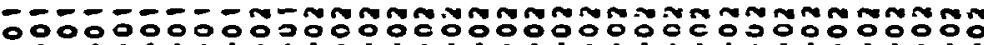

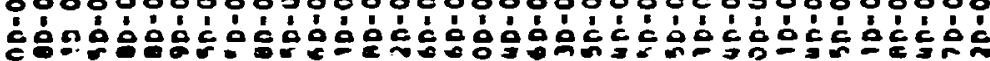

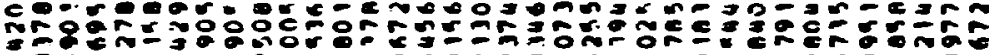

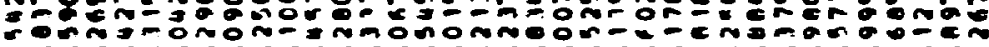

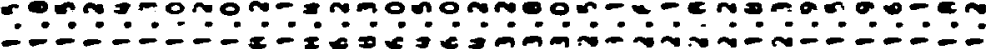

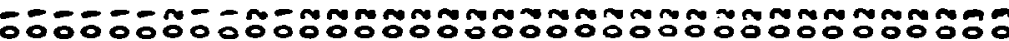

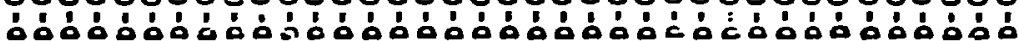

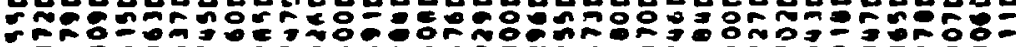
- mo Z:

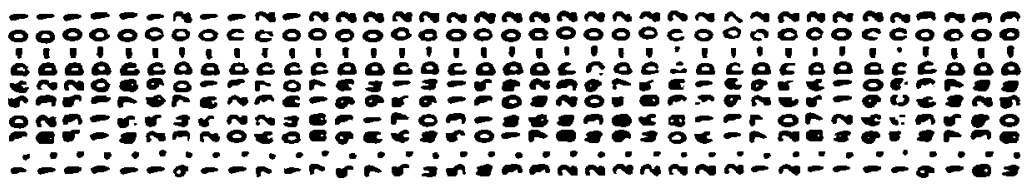

¿́íd

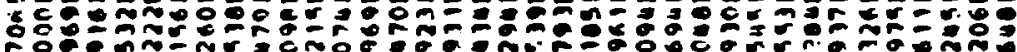

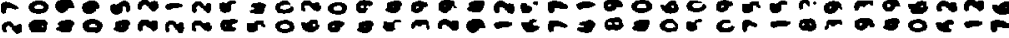

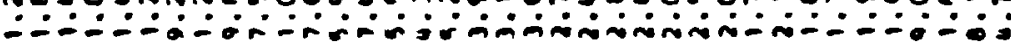

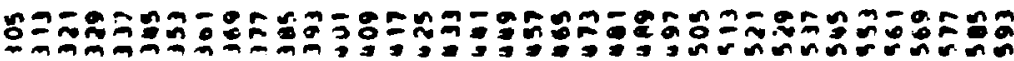


APPENDIX C

LISTING OF DLSSOLVEDCONSTITUENT TRANSPORT CODE 


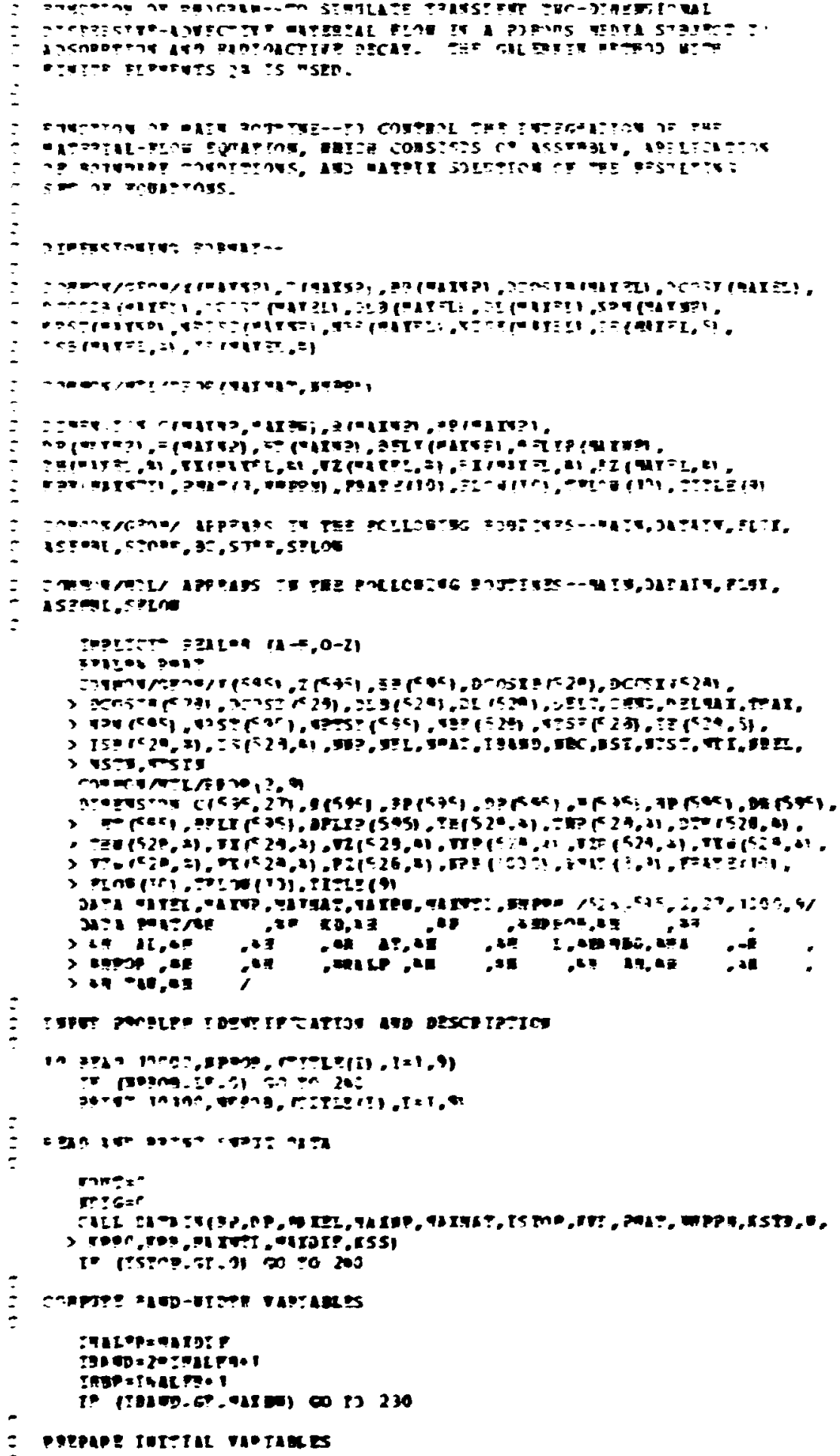

perpape rotoral vaetaces 
$+n==$.

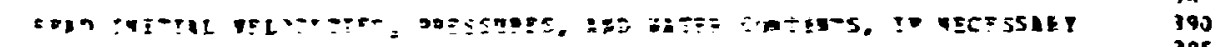

an $20=1, \forall=1$

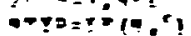

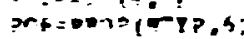

$\because-2 n=2=1,2$

Cof

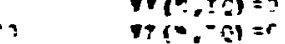

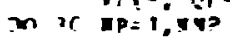

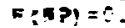

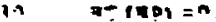

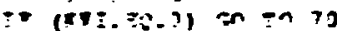

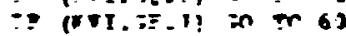

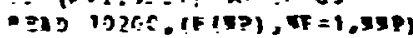

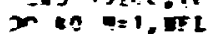

x-

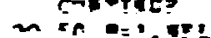

$0.2 \times 1,4$

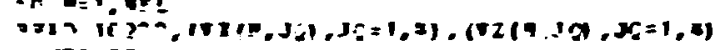

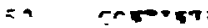

$=\div 19$

A-

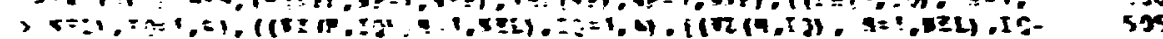

$21,11.0,100102,519$

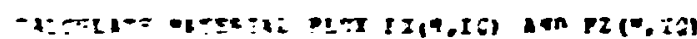

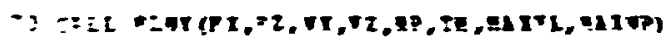

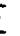

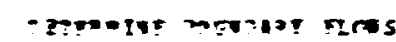

ac mat $t=1,4$

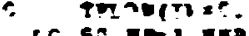

e) citiopi=?

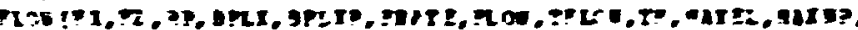

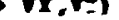

$n 19 \% t=1,0$

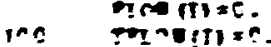

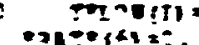

$650+1,1=-2$

$20,0101=0$

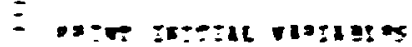

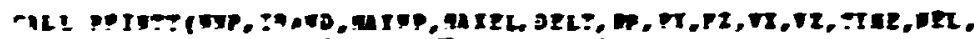

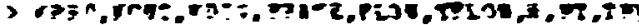

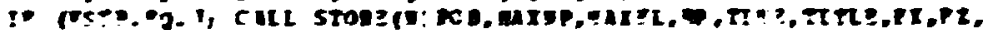

$>,+, 1 x_{1}, 1$

$=$

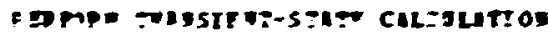

iTQEDITt

130

$1=1,-0$

on $220+T=1, \pi T !$

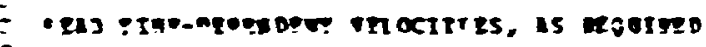

$x$ in wp=1.0\%

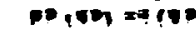

$x$ in the $1, y=1$

on $1,0 \quad ? 2=8$

$-\pi(4, \div 3)=9(4,10)$

ITP (P.,

vet $(0, i g)=2(n, 10)$

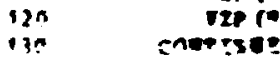

T* (CVT.RE. $(-n)$ co T) 160

prab inzo, rrion, ope!, gun

m ist neigel

pro inz 30 , (n) $(n, 10), 10=1,4$

$\mathbf{a t a}$

$\infty 05$

$=15$

20
$\div 25$
.30

30

ax

305

750

35

565
+72

$+75$

yes

$9=$ 


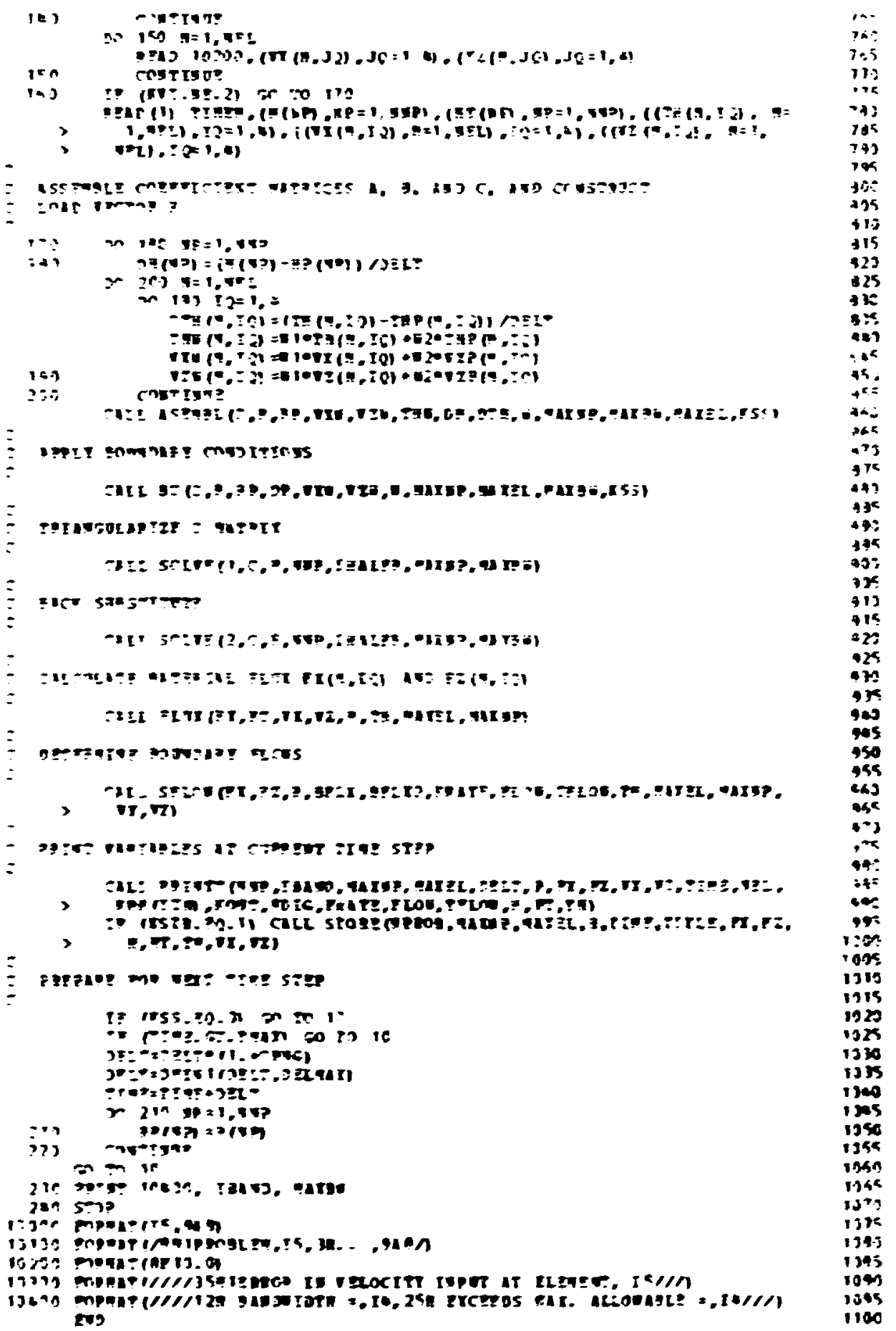




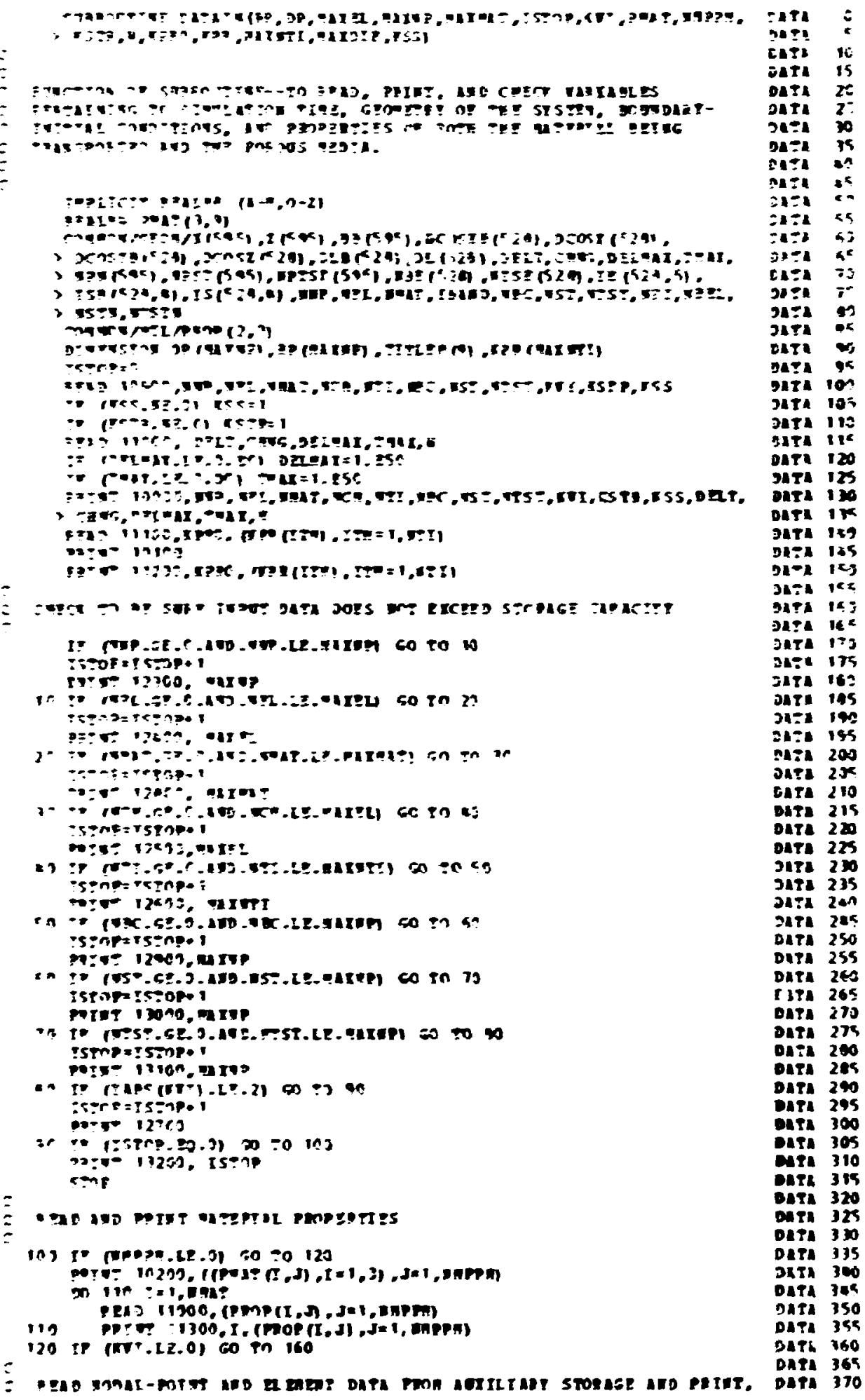




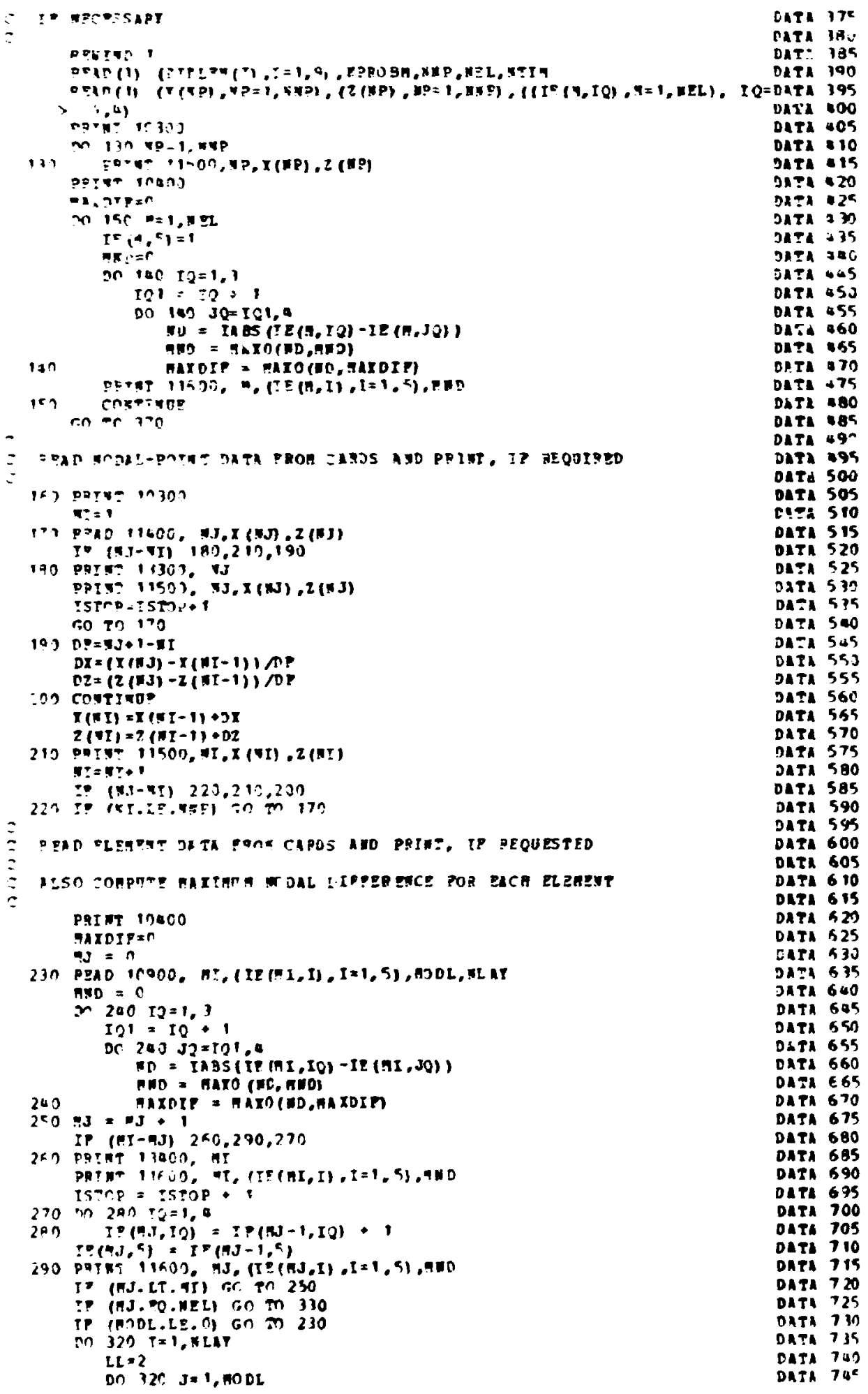




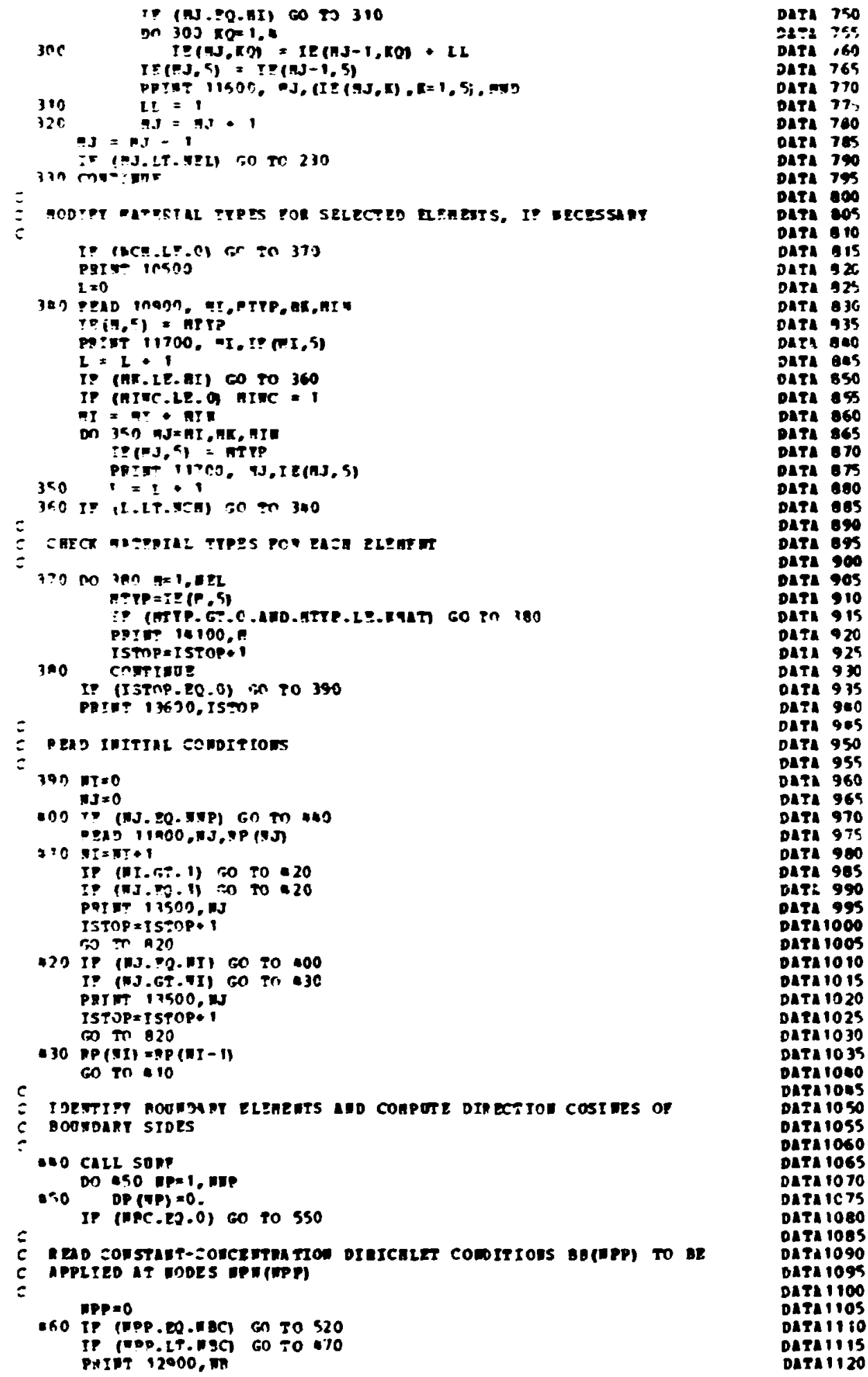

Data 750

$28=2$ ?5:5

DAta 160

DATA 765

Data 790

Data T7,

Data 780

Diri tes

Data 790

Ders 79

Data aco

Dira 205

Dara 10

DAsh 15

Det1 $2 x$

DArA 2 ,

DATa 836

Data 95

Data gao

onta ans

data 850

oAta 29

Data 860

Dira 865

DAT1 670

Data ets

DITI 680

DAra 005

DATI 89

pata $\mathrm{ES}$

DLTI 900

Data 905

DerI 910

DETI 915

DATA 920

DATI 929

DATA 930

DLTA 975

Dera 9e0

DATA 9.5

DATA 950

DATA 955

Data 960

DATA 965

DLTI 970

DAT1 975

DATA 900

DATa 905

DAT: 990

DATA 995

DATi1000

DAti 1005

Data 1010

Data 10 is

DATa 1020

DAesiozs

Dataiojo

DATA 1035

Data ioso

DAThions

DAF 1050

DATA 1055

oaraloso

Data 1065

Dati 1070

DATIIC75

DATA 1080

OATI 1085

DATA1090

Dari 1095

DAT1 1100

Daralios

DATIIItO

DATIIIIS

Daralizo 
teter $=: 2 m p$

in $-n<20$

4- 0 DIS 11000,5 I, HPIMC, Bat

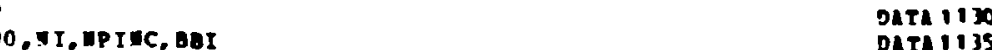

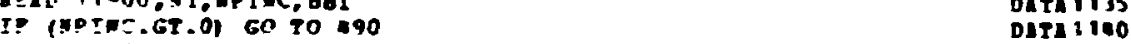

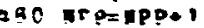

EPY $(n \times p)=M I$

$B P(T P D)=351$

Go Tr se0

Q99 TF (MTO.GT.0) 60 Tn 500

ISTOP $=$ T 2 TOP.

patst 13900

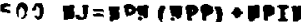

SPJ $=$ PA (NPP)

$M K=R T-1$

DO 510 mexis, $3 \pi$, Der:

$Y F P=M F P+1$

$\triangle P$ (NPP) $=$ IP

$80(19 P P)=0.3$

co TC man

$52 n$ pp:Tt in600

D.) $<36$ จ $P P \approx 1, \nabla B$

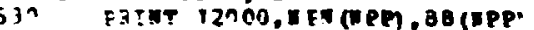

Dara : 185

DATA I IS

Eath 1159

Dataing

DATAI 165

DATA 1170

JATA 1175

DATA IBOC

TRTA 19S

D. 11190

DATA 1195

DATA 1200

DATA 1205

DAT 1210

DATA 1215

DATA 1220

Dara 1225

Data 1230

Darh 1235

Deta 1240

DATE 1245

DAT:1250

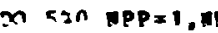

- $P=\triangle P$ (T)

C.0 FP (NO) = 0 (GFP)

5KD I: (NST.LE.9) GO TO 650

5

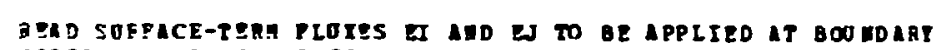

DATA 1260

DATA 1265

DATA 1270

DATA 1275

DATA 1290

DATA 1285

DATA 1290

DATA 1295

Dara 1300

Dara 1305

DAFA1310

Dara 1315

DATA 1320

DATA 1325

DATA 1330

DATA 1335

DArA 1340

DATA 1345

DATA 1350

DATA 1355

DATA 136 \%

DATA'j65

DETL 1370

DATA 1375

DATA 1380

DATA 1385

DATA 1390

DATA 1395

DATE 1400

DATA 1405

DArhis 10

DATA 1915

DATI IA 20

DATA 1425

DATa is 30

DLTA 1435

DATA 1440

DATA 1845

DATA 1850

DATA 1455

DATA 1960

DATA 1465

DATA 1070

DATA 1475

DATA 1490

DATA 1485

DATA 1090

DATA 1995 


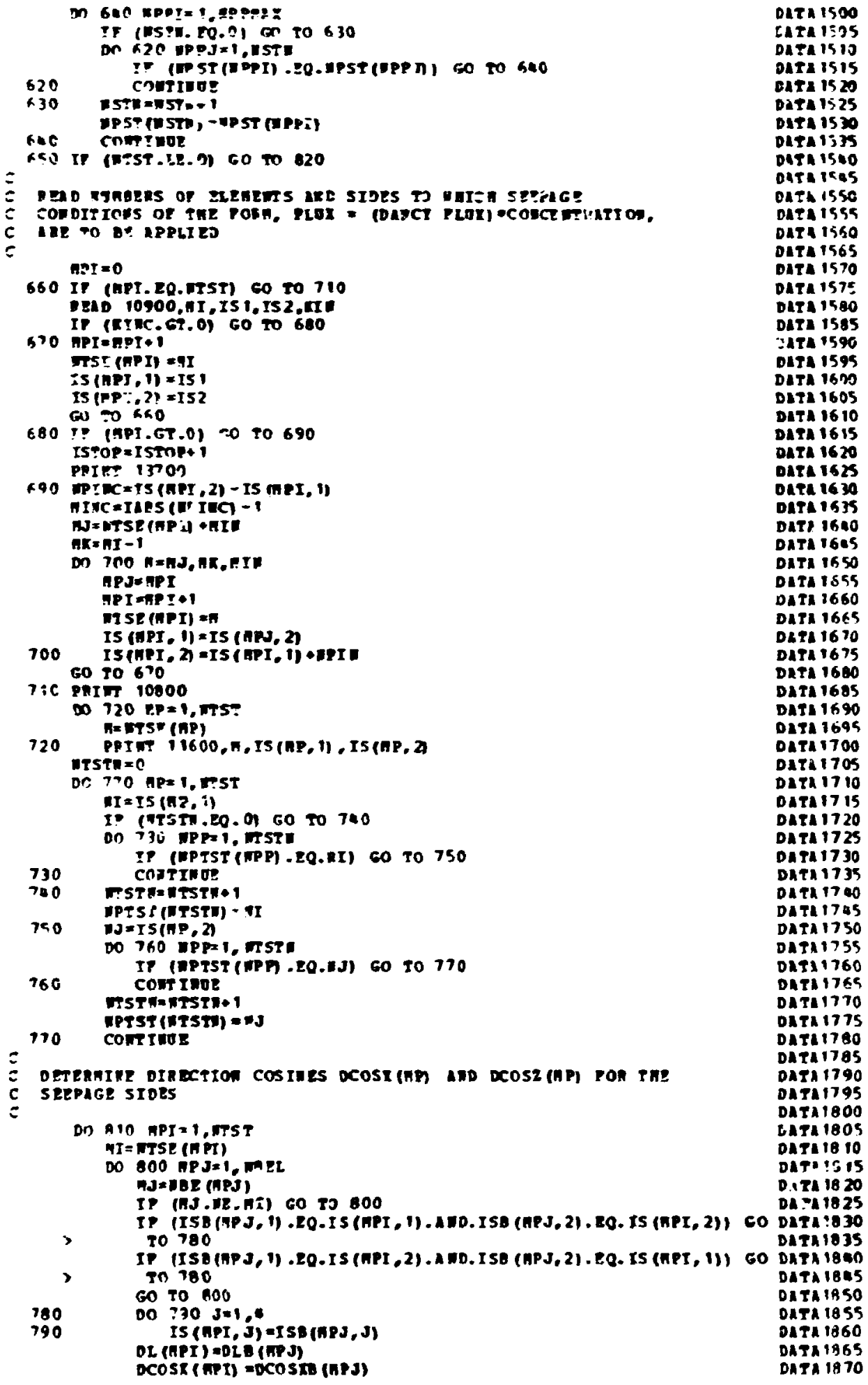




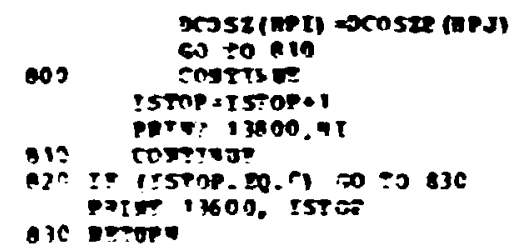

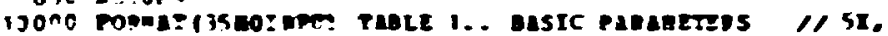

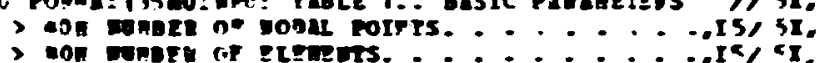

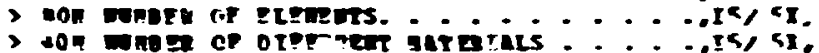

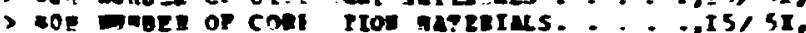

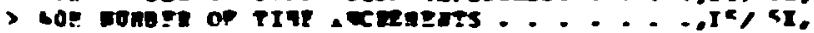

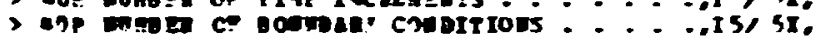

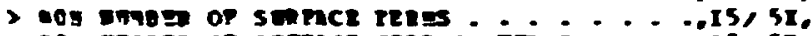

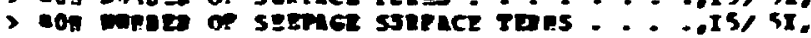

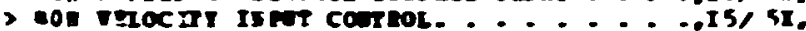

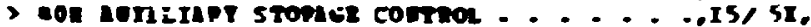

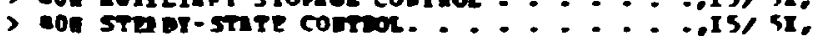

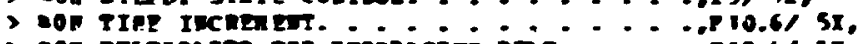

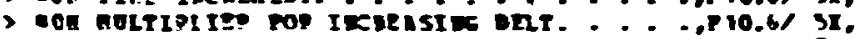

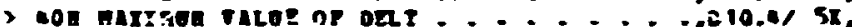

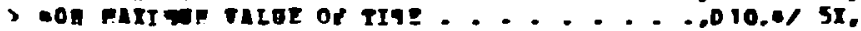

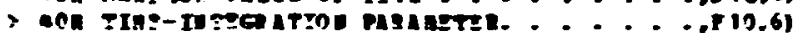

Desistex

D) 1980

Dara iees

Drti 1490

Dera 185

Dara 1900

DATI 190

GeTh 14 10

Daraigis

eata 1920

จง 1925

Deri1930

DATA 19 35

DATL1940

aralies

DArat950

DAF 1955

כura isco

DATA 1965

כ9 1970

BrTE it

cati iseo

DAra 1955

Dara 19so

DATI 1995

Datn 2000

Daruzos

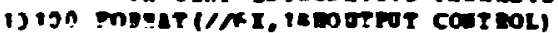

arrizeis

$3(34)$

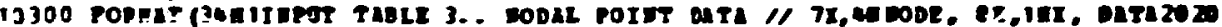
, 1ex, int)

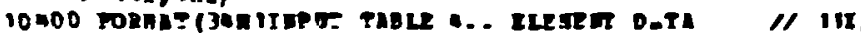

DIT2025

01212030

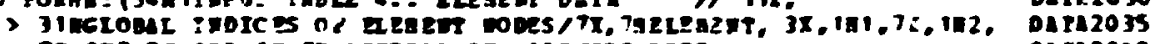

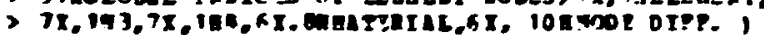

DA r 2000

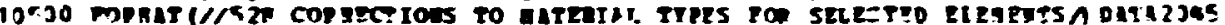

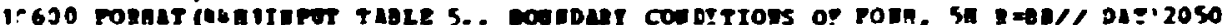

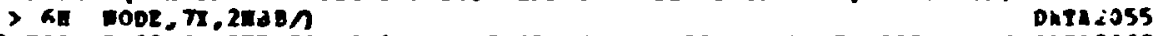

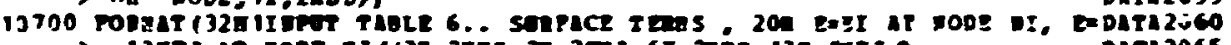

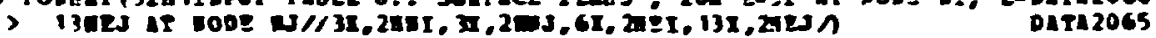

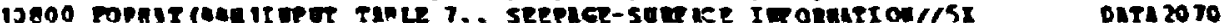

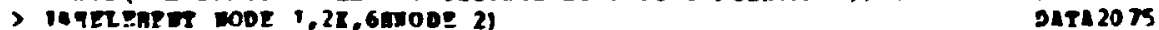

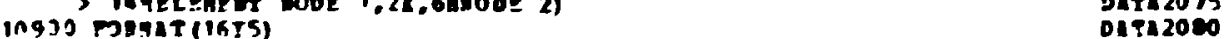

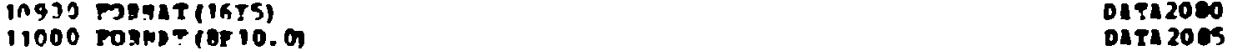

$\begin{array}{ll}11100 \text { manat recti) } & \text { 0arazoso }\end{array}$

11290 mpha-(10x,101?11

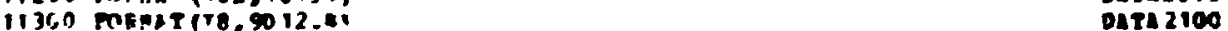

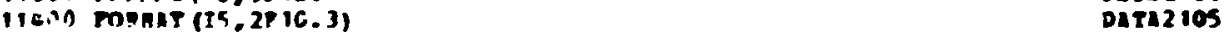

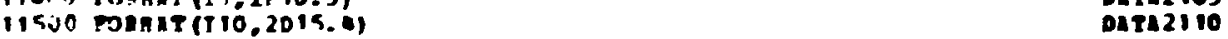

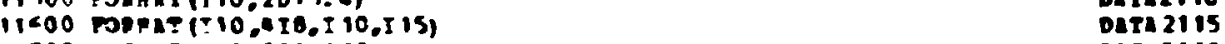

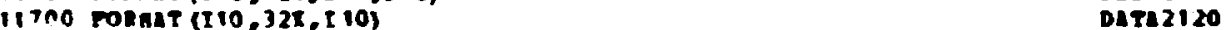

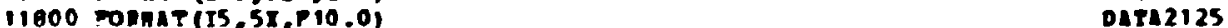

11900 ponntisis, Pig.nis

$\begin{array}{ll}120 n o \text { ponhat }(T 5.015 .0) & \text { DATA2135 }\end{array}$

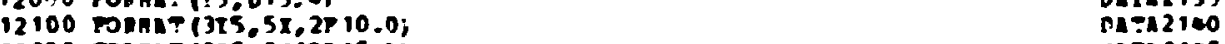

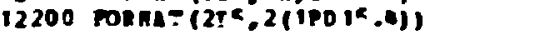

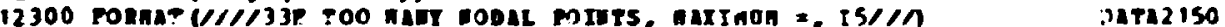

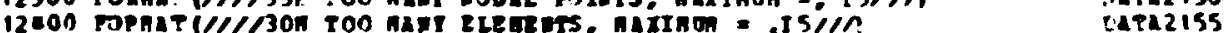

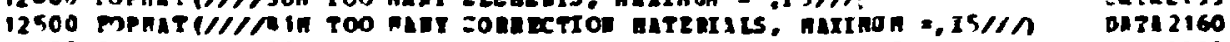

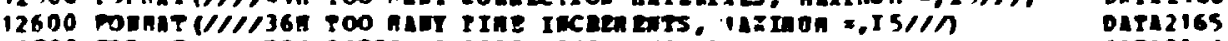

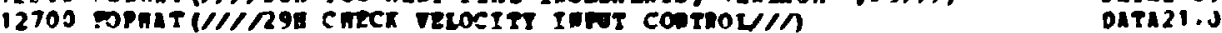

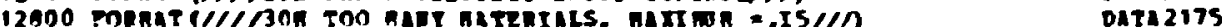

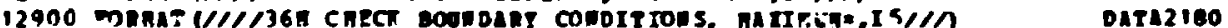

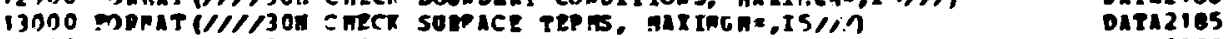

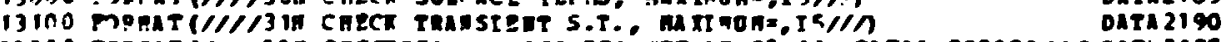

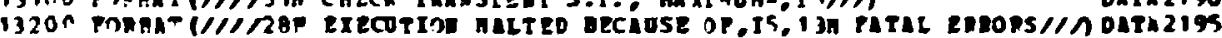

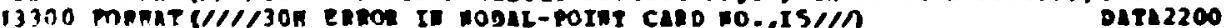

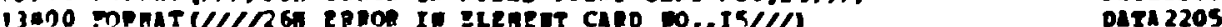

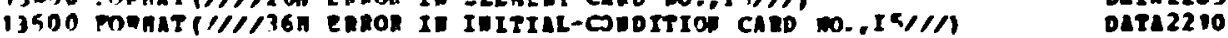

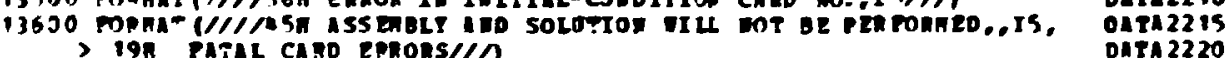

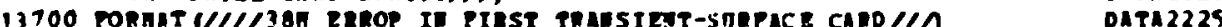

13800 mRHT

13900 ment

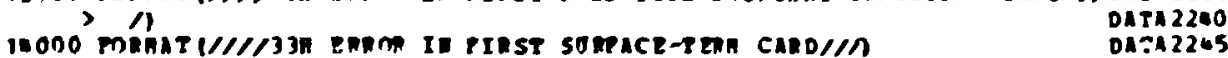




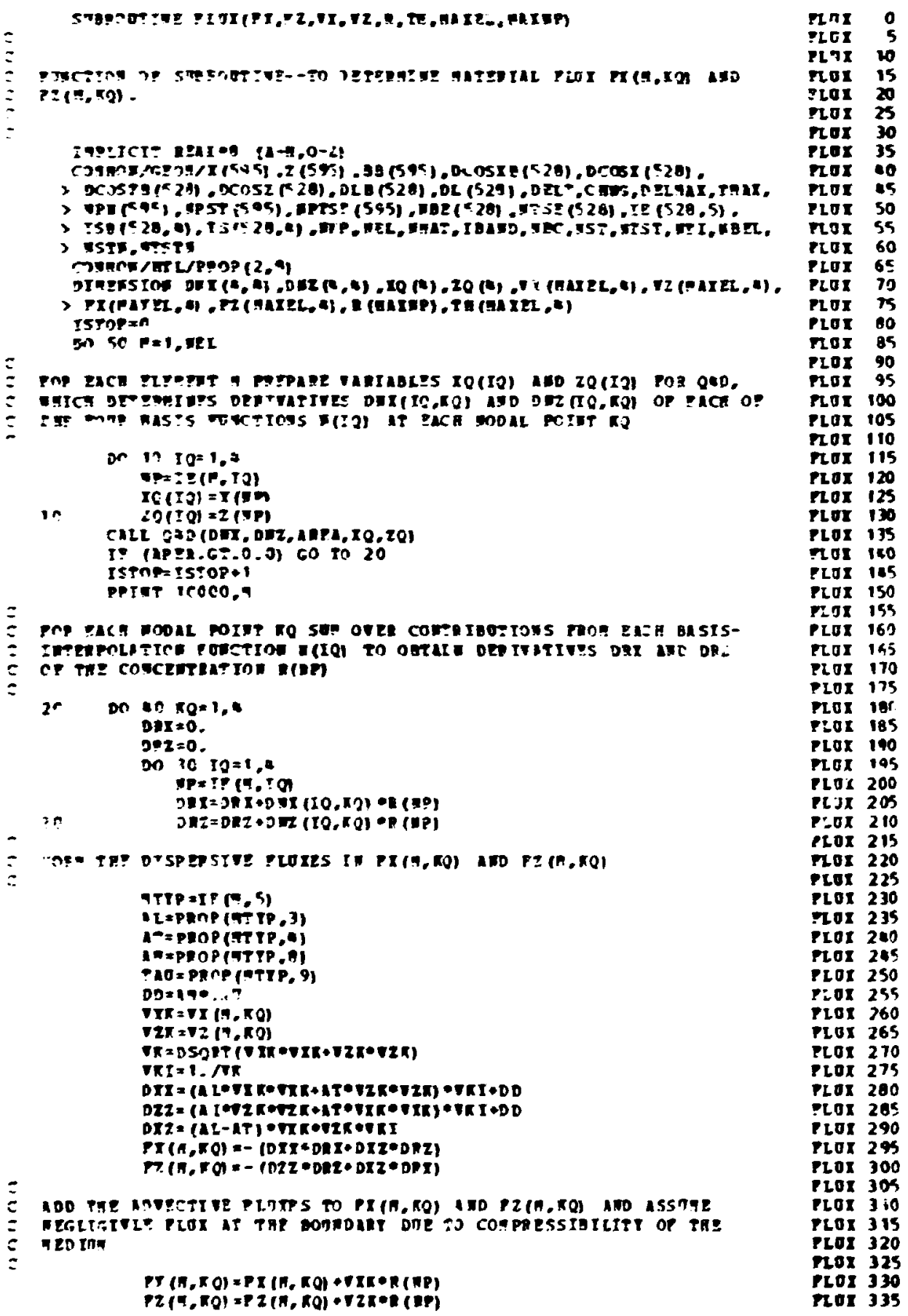


$10 \operatorname{cosin} 0$

conprove

IP (ISTOP.ST . 0) sTOe

atoro

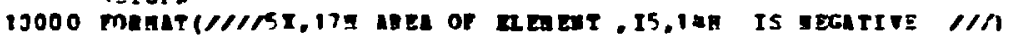
IDD

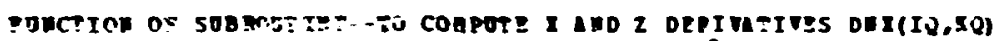

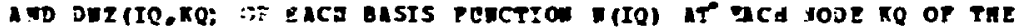
ELEPDT. FESOLTS ARE IN TRE GLOBL COOBIMTE SISTE.

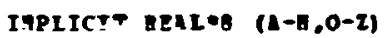

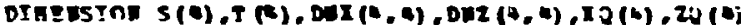

PATA $5,-1.00000,1.00000,1.00000,-1.00+00, T,-1.00 * 00,-$ $>1.00+0 n, 1.00+00,1.00+00$,

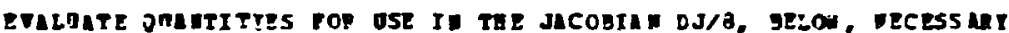

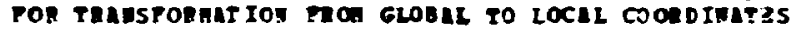

$112=10(1)-10(2)$

$I 13=x Q(1)-x 0(3)$

$123=\mathrm{xQ}(2)-\mathrm{T0}(3)$

I10 $=x 0(1)-x 0(4)$

$x_{24}=\mathrm{xQ}(2)-\mathrm{xO}(\mathrm{e})$

$139=x Q(3)-10(4)$

$213=20(1)-20(3)$

$220=20(2)-20(4)$

$230=2 n(3)-20(4)$

$212=70(1)-20(2)$

$223=7.0(2)-20(3)$

$210=20(1)-20(4)$

AQ2A $=713 * 22 e^{20(2)}-120 \cdot 213$

$\succeq$ LOOP JVE EACB WODE

on $10 \quad r g=1$.

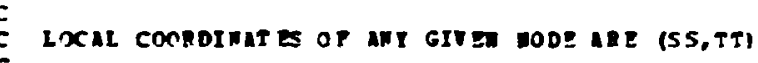

$s 5=s(n 2)$

$T=T$ (TO)

evalohte "acostan"

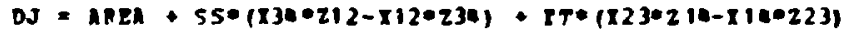

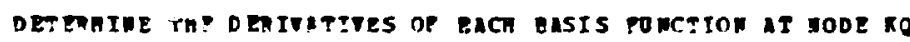

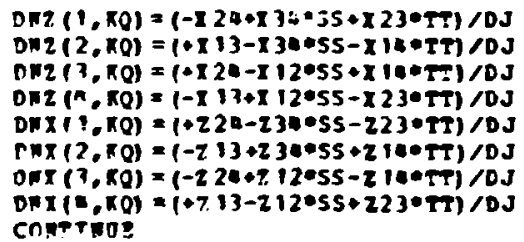

2000

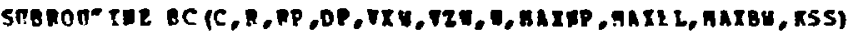

$\begin{array}{ll}\mathrm{BC} & 0 \\ \mathrm{BC} & 5\end{array}$ 


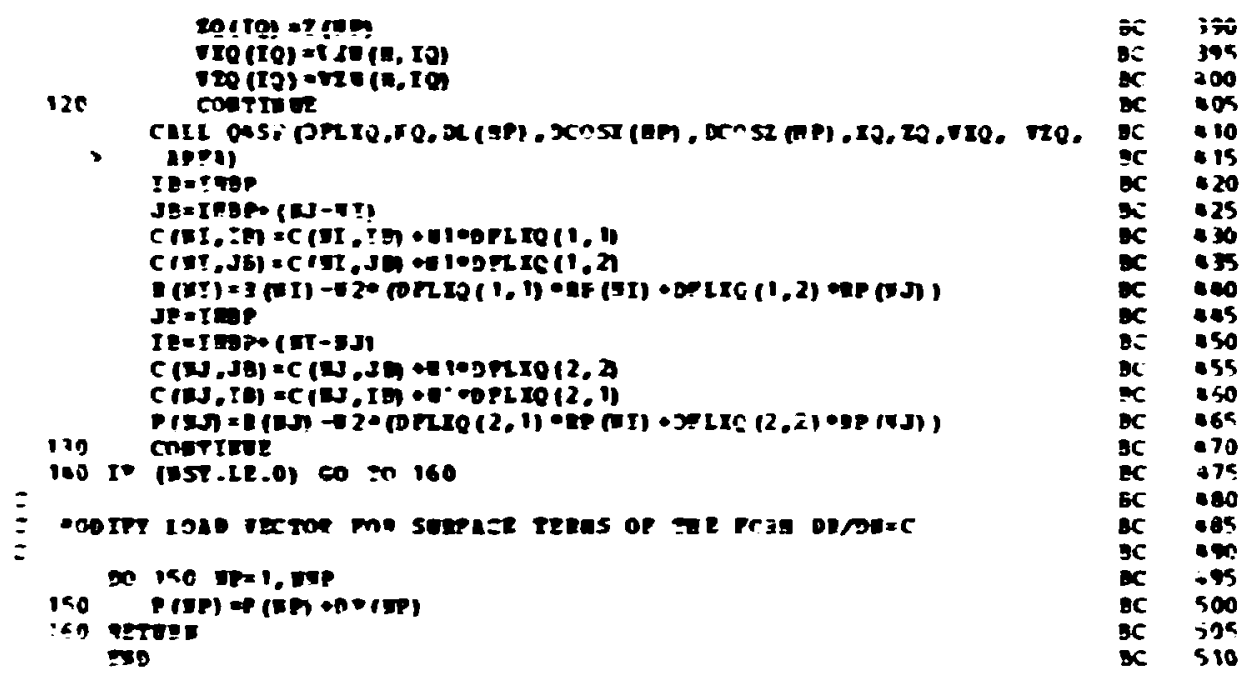

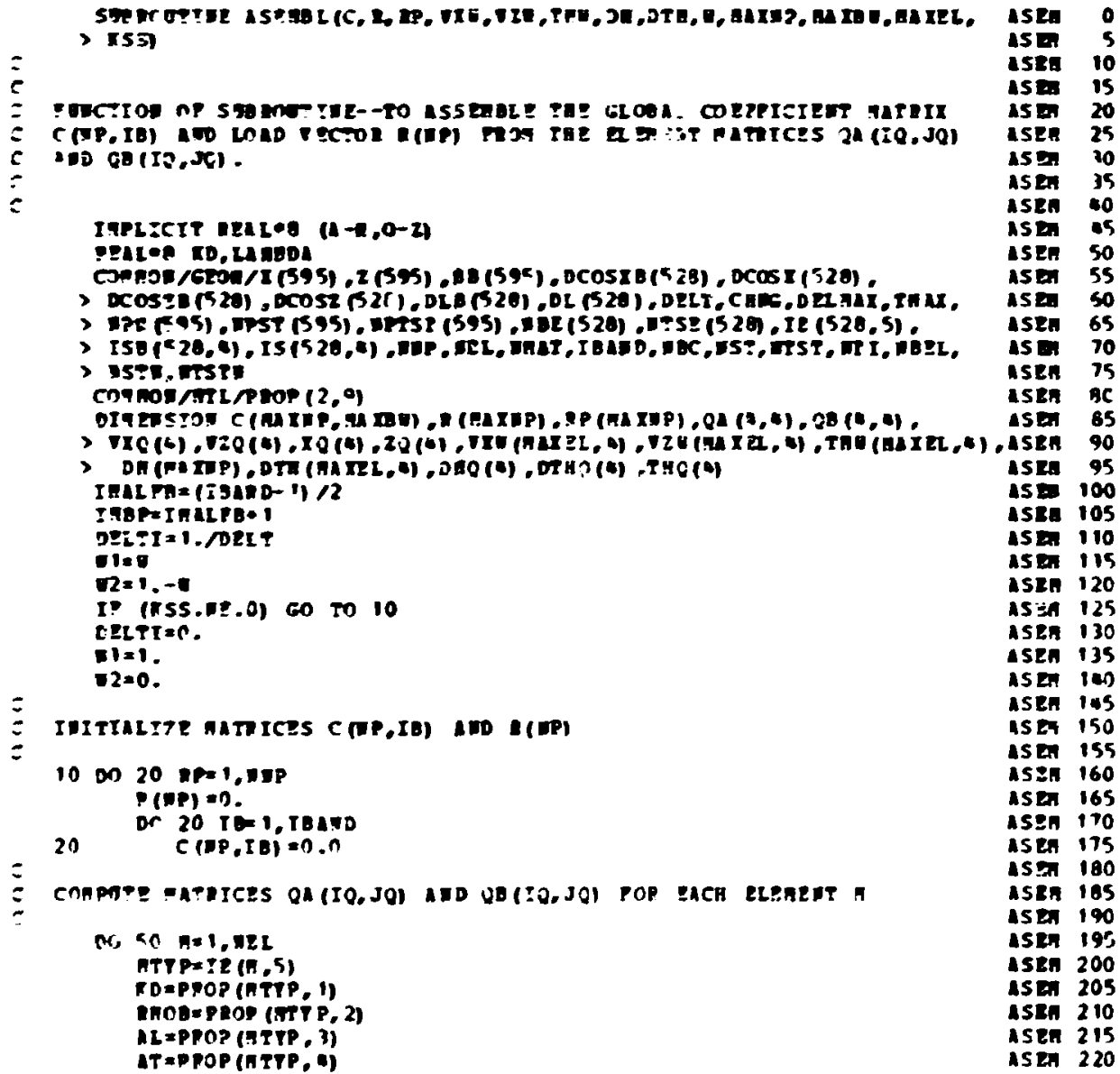




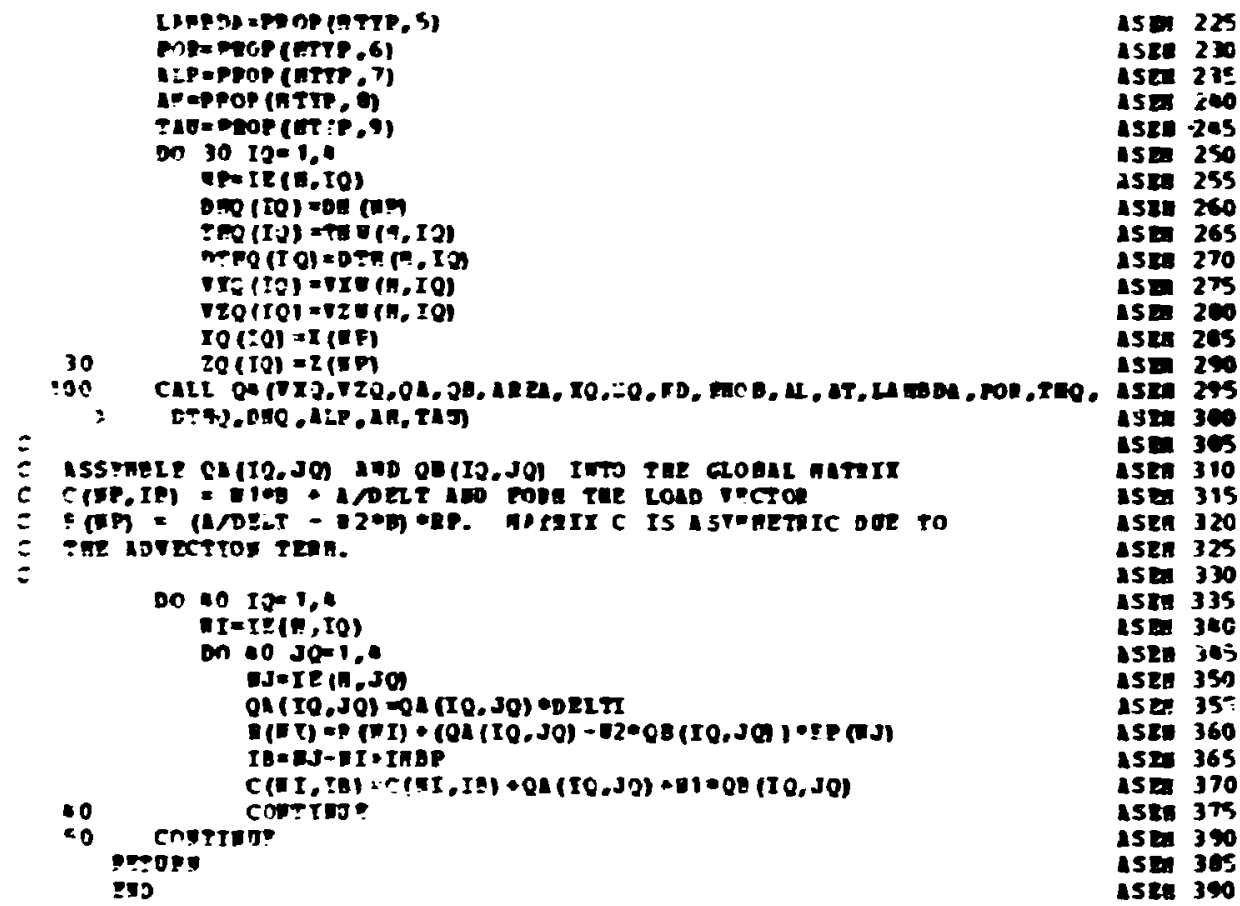

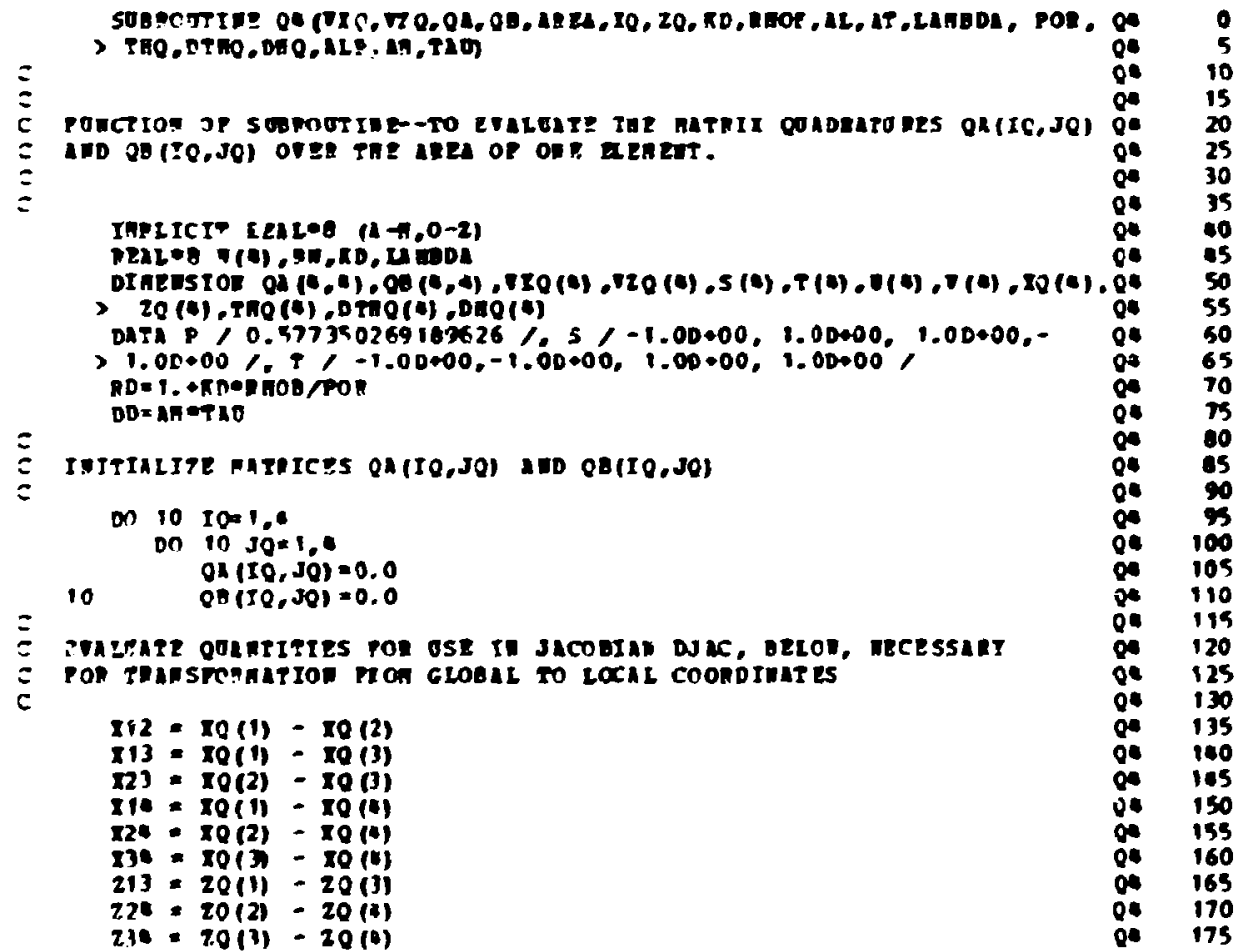




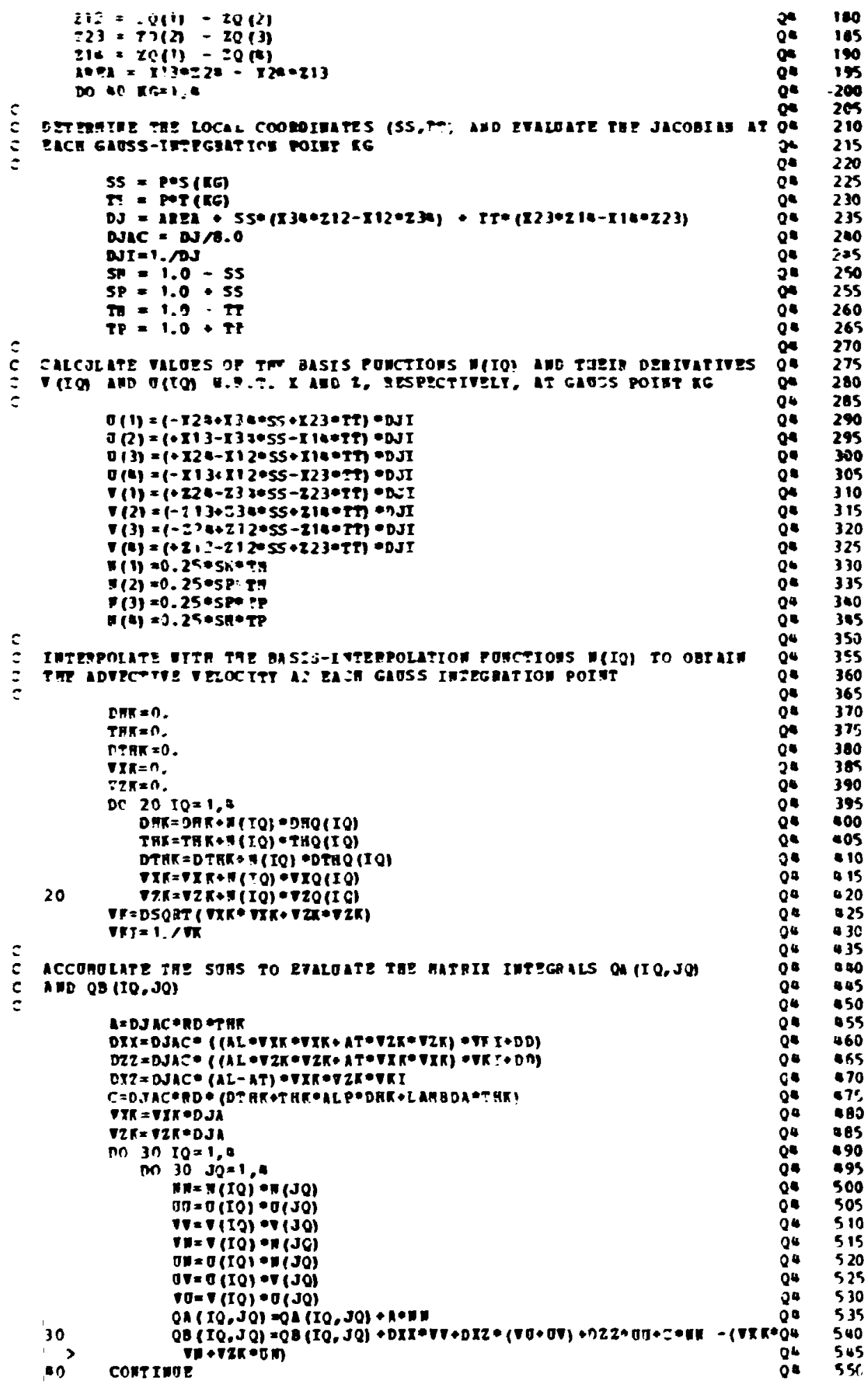


DC TR $I T=1, \pi \pm P, 8$

$\operatorname{sen-4I}$

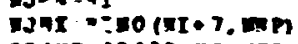

7

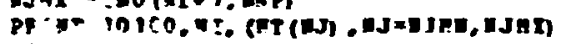
ET:E:

I)

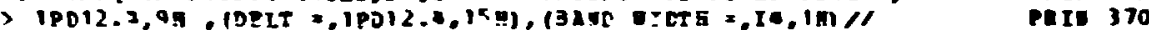

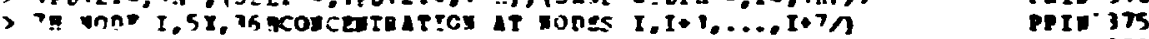

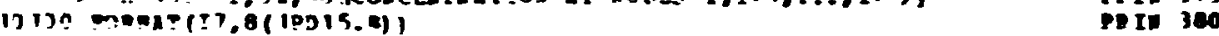

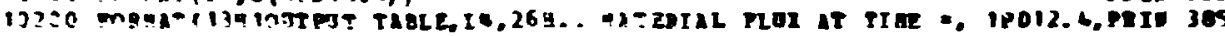

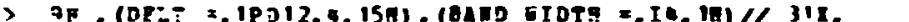

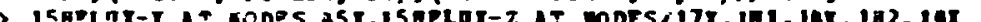
PEIV 39

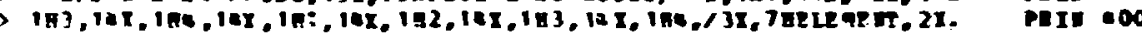

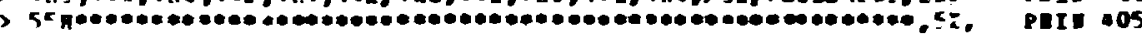

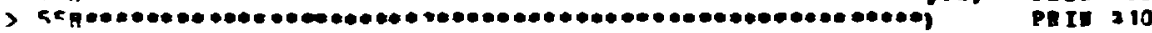

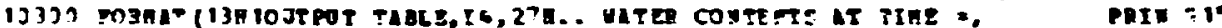

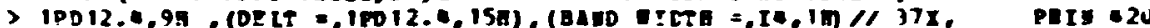

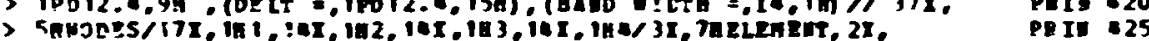

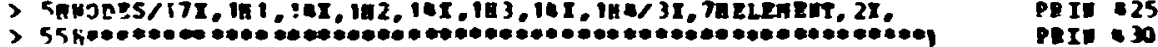

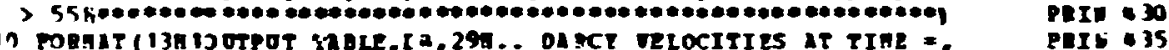

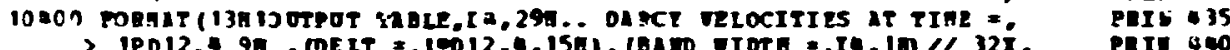

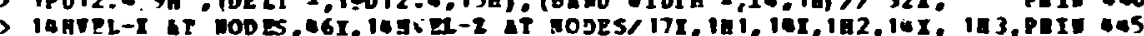

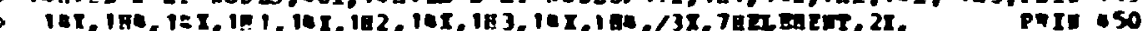

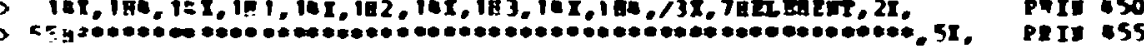
; Kर

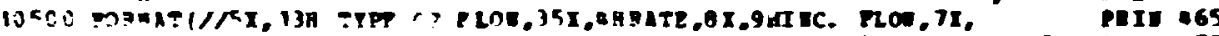

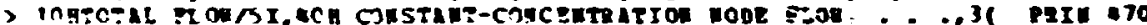

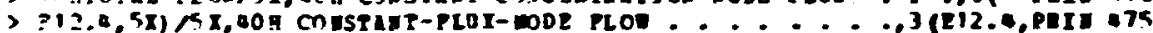

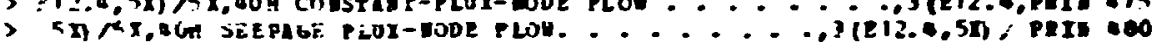

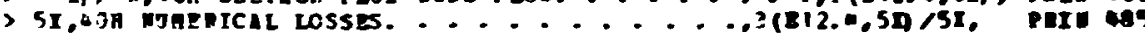

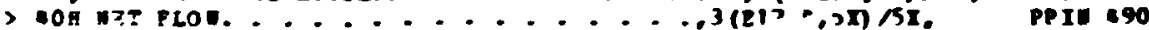

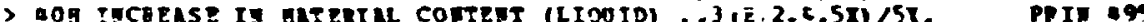

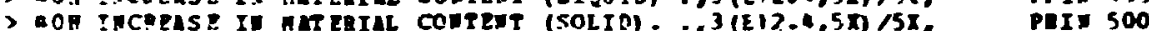

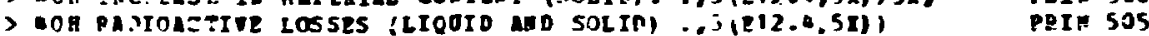

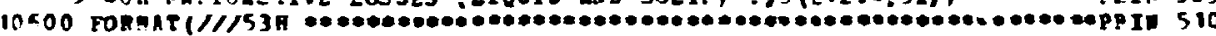

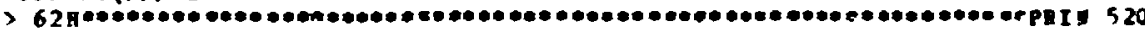

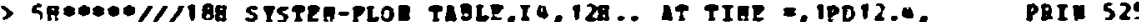

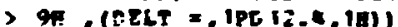

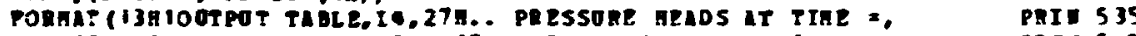

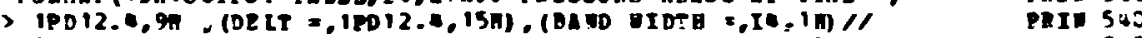

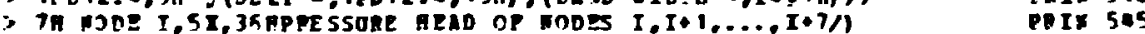

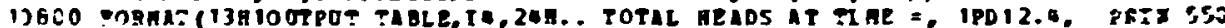

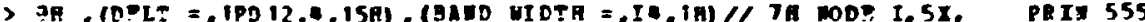

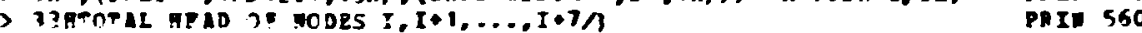
Q.⿴囗十

IAP LICIT REA LEB (A-, $0-2)$

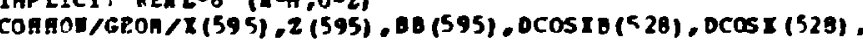

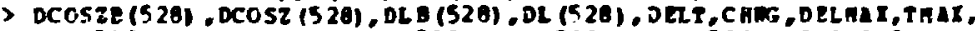
ME (595). MPST (595), MPTST (595), DBE (528), DTS (526), IR (528, 5)

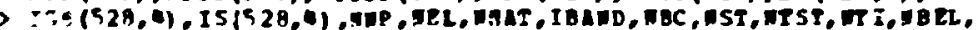
ThTH,Nest\%

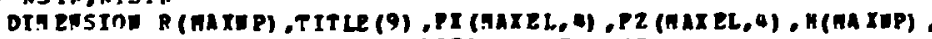

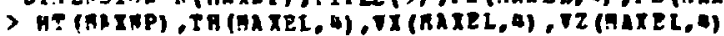

DATA TPPROB/ - I/

IP (NPPPOE.RQ.(-1) IEUIND 2

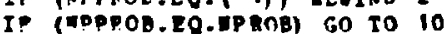

URIT:(2) (TITLE(I),I=1,9), MPROB,ANP, NEL, NT I

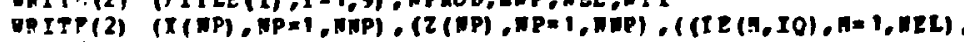

$>I Q=1,(4)$

DPPACB= DPROB

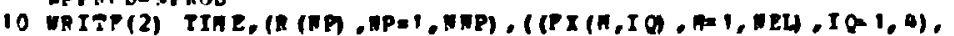




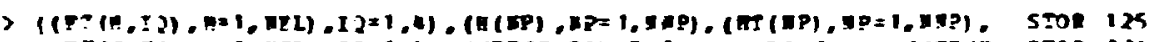
) ( TET

STOR 160

Q15

$5=01145$

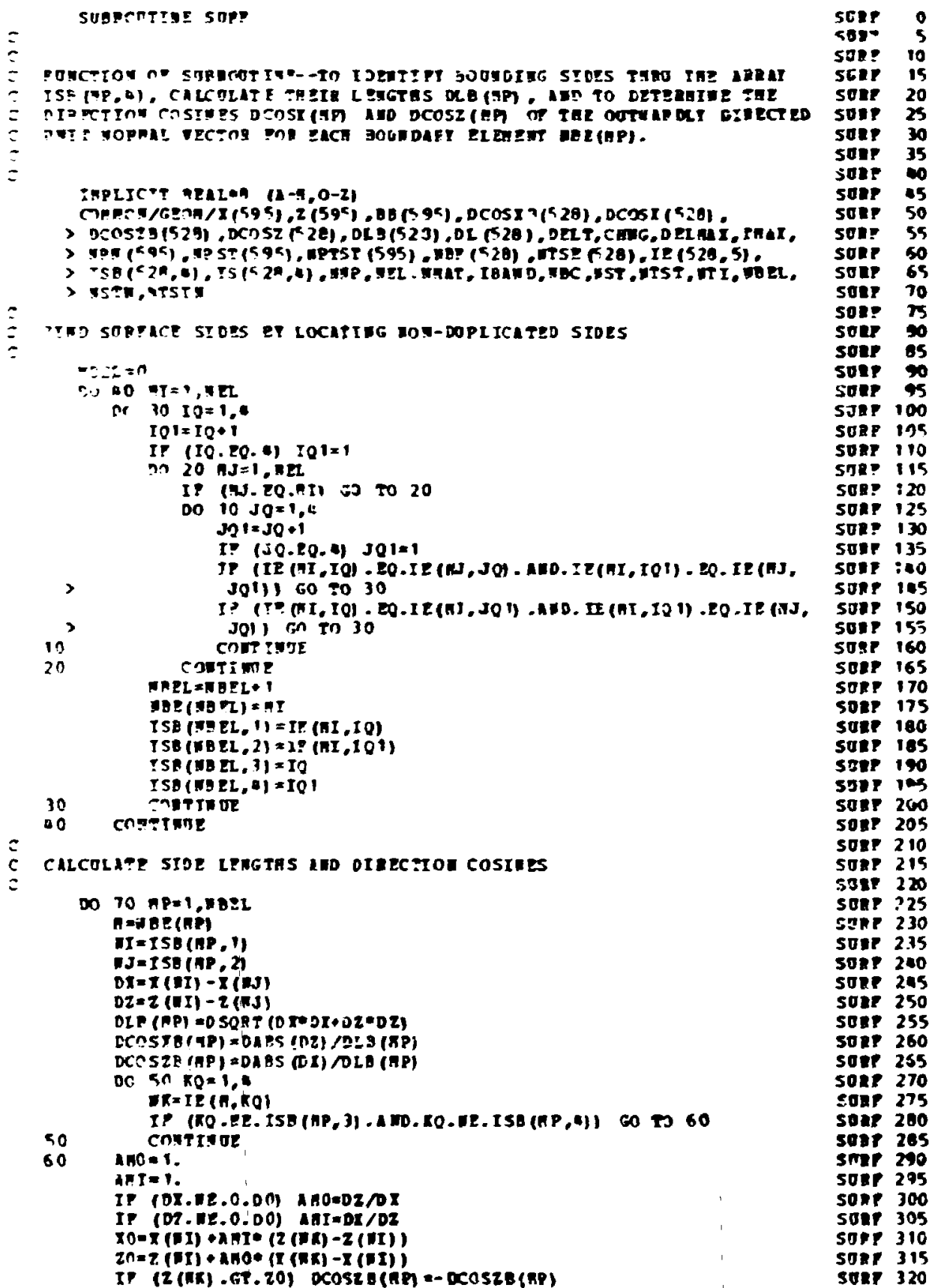




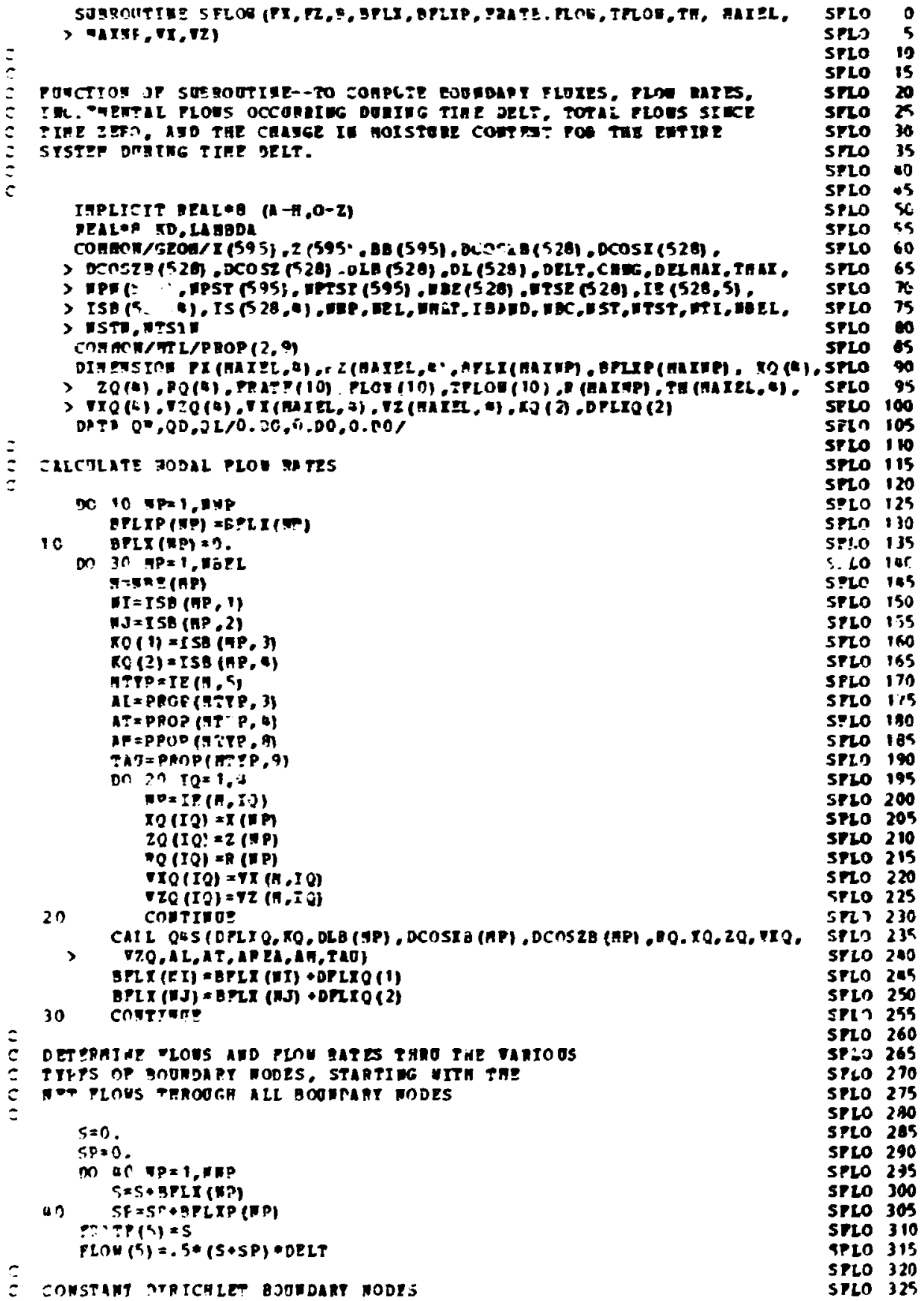




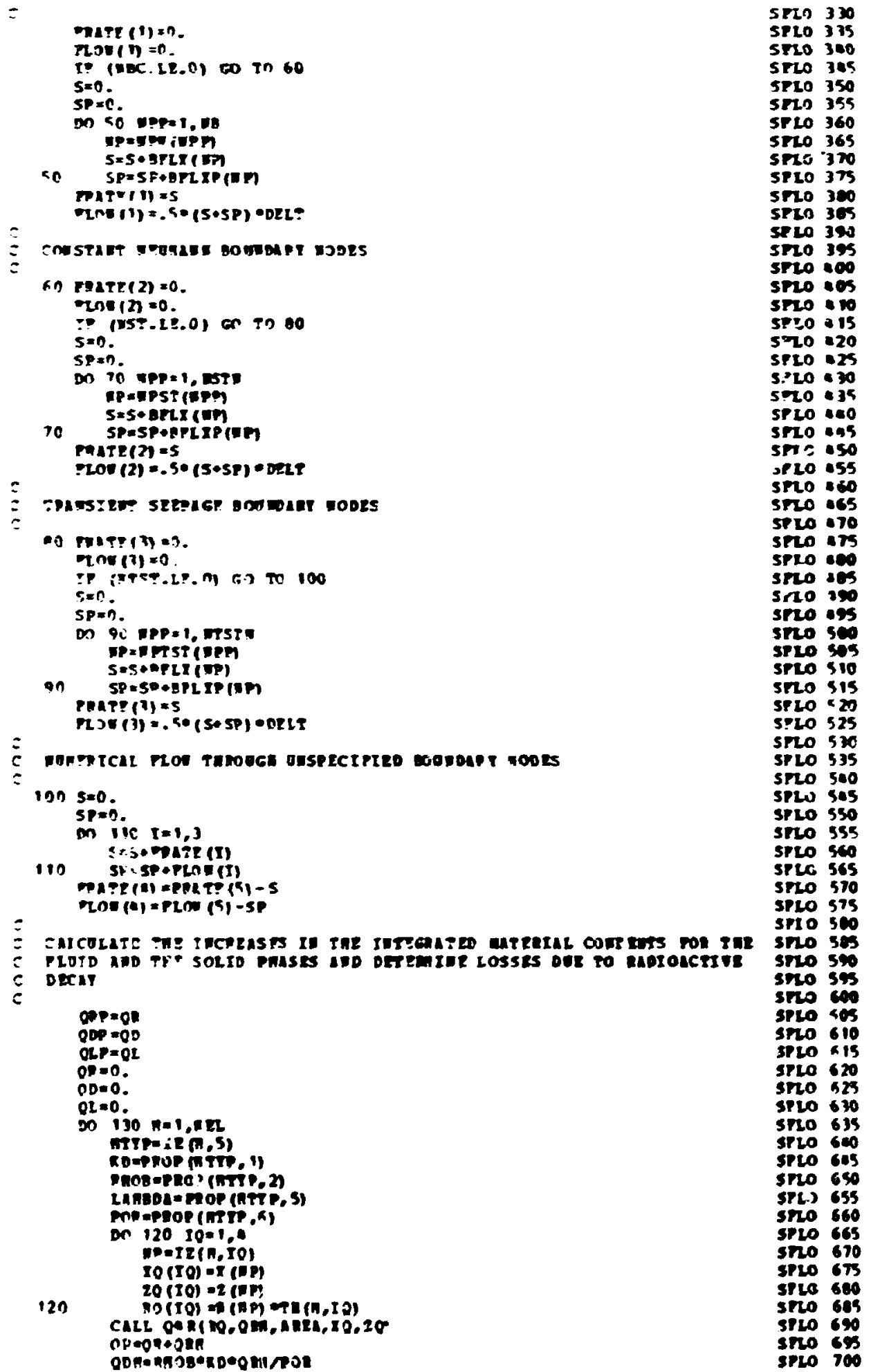




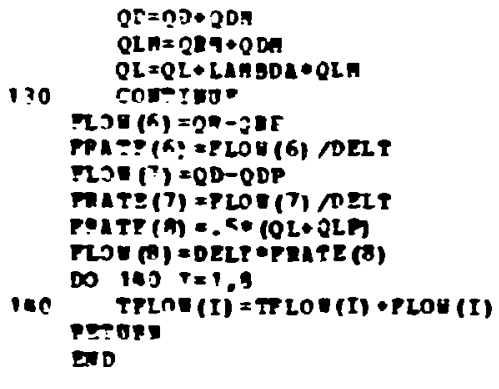

Ero 106 Si 20710 $S ?-0715$ STLO 720 5720725 $5 P 20.740$ STLO ins SFLO 750 SPLO 755 SFLO 760 SPLO 765 SPLO 770

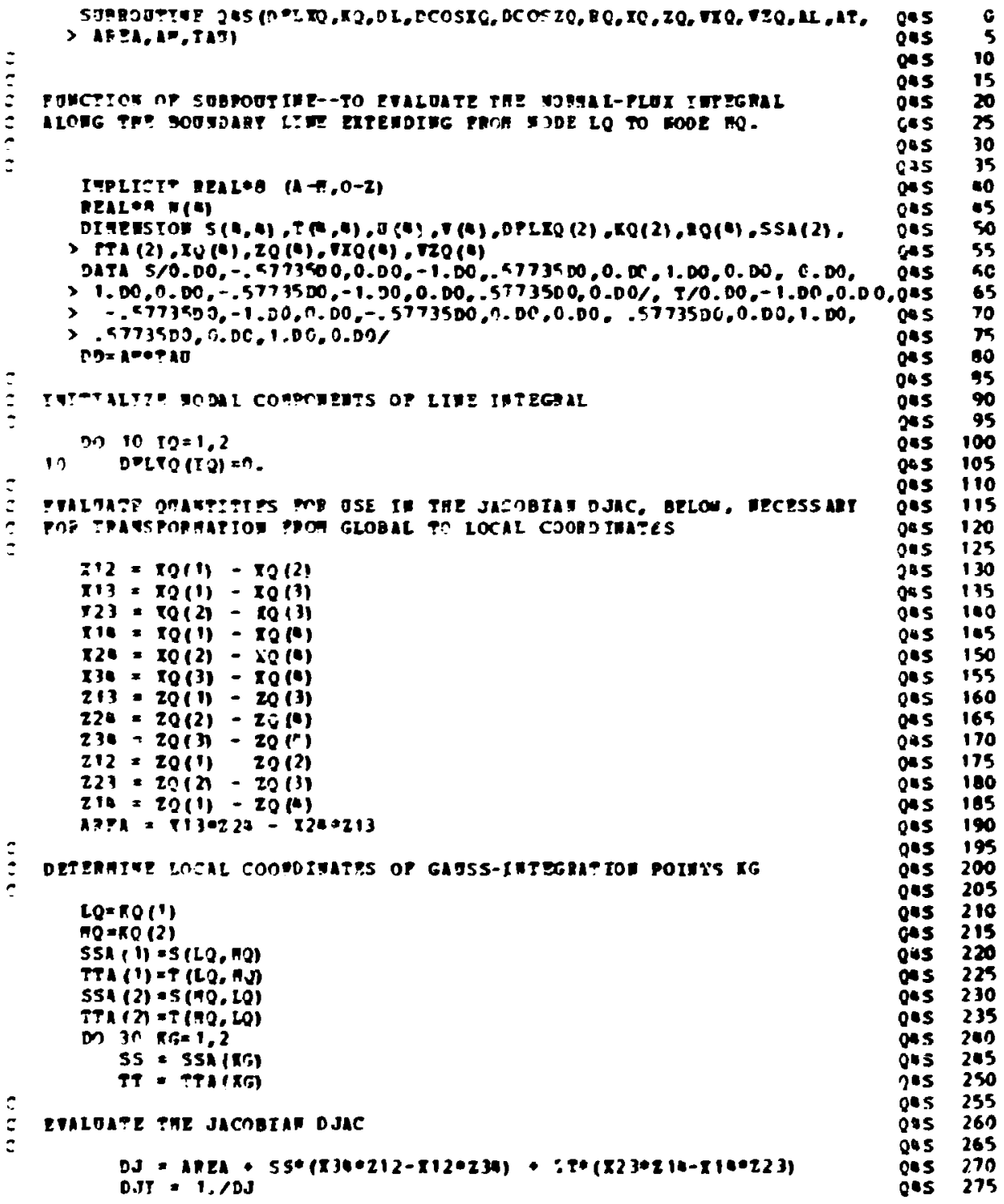


NAC = .12509J $\quad 045 \quad 200$

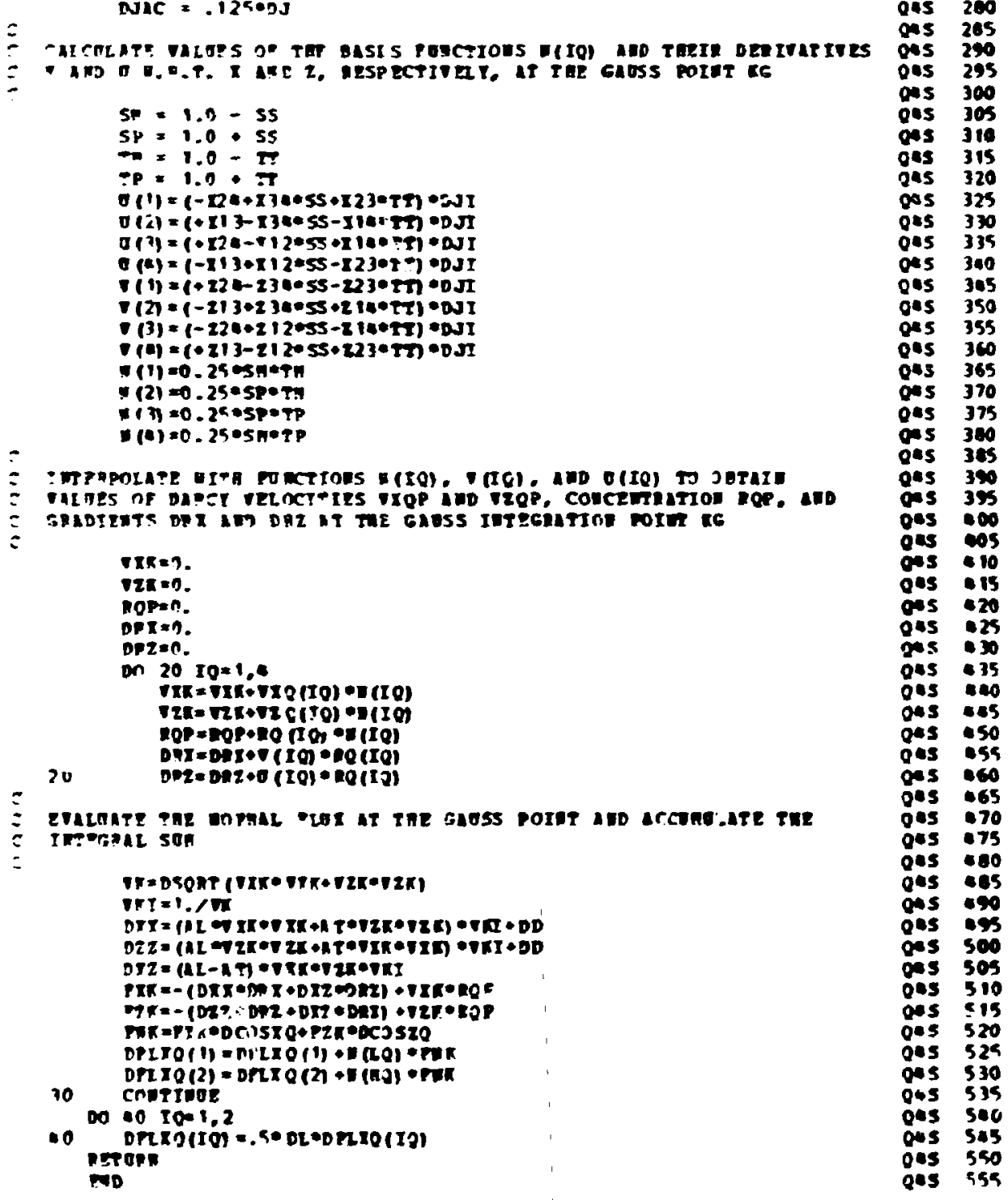

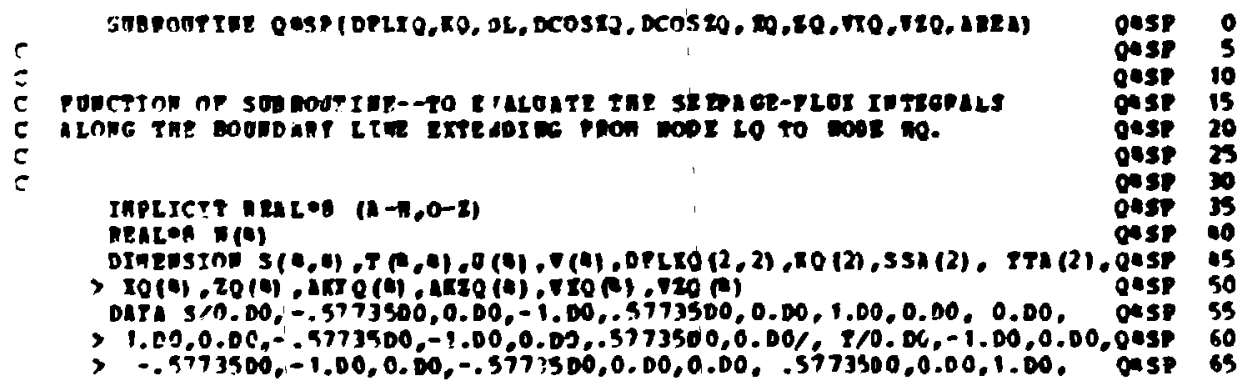


$>.5773500 .0 .00 .1 .00 .0 .00 /$

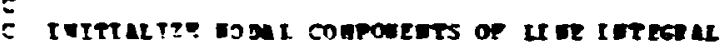

$m 10 \mathrm{JQ}=1.2$

m $10 \quad$ I0.1.2

$10 \quad 3=2 \times 01 \mathrm{TO}, \mathrm{JM}=0$.

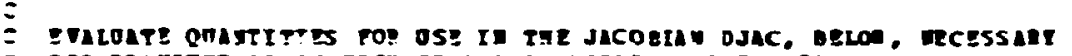

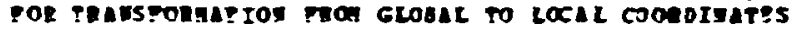

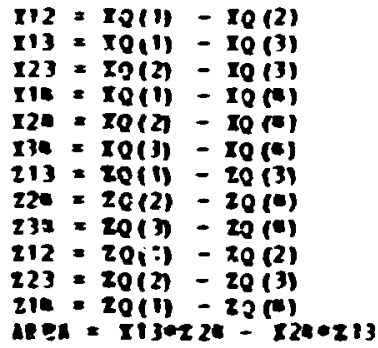

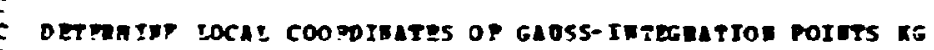

$t o=(20(1)$

$\cos =\operatorname{Re}(2)$

$\operatorname{Sin}(1)=5(20,5)$

$\rightarrow 1(1)=\mathrm{T}(\mathrm{LQ}, \mathrm{Fa})$

SSA $(2)=5(90,20)$

$\pi A(2)=-(90,10)$

no $0 \quad k+1,2$

$55=\sin (\pi \sigma)$

$T T=\operatorname{Tr}(\pi G)$

Frazote the jacosing ejar

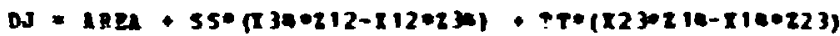

DJ1 $=1.10 \mathrm{~J}$

DJAC .125 ०DJ

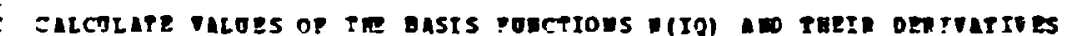

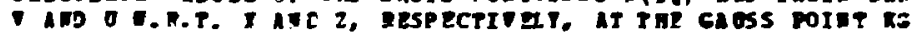

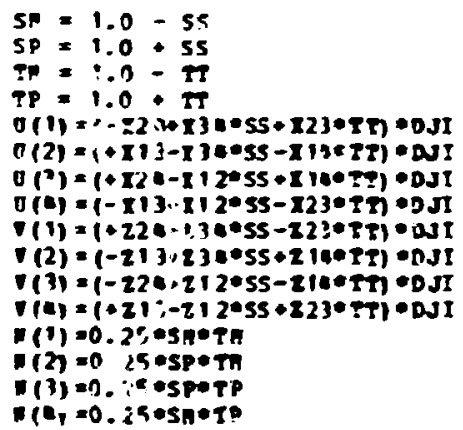

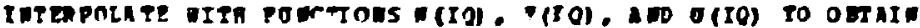

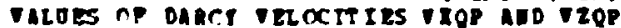

$\boldsymbol{V I F}=0$.

vin=n

on $2912=1.0$

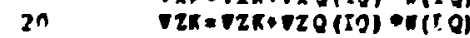

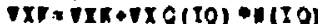

5asp 70

jesp 75

gesp 0

Qusp as

Qesp 90

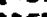

Qesp 105

Q*se 110

oesp 115

ous? 120

Qesp 125

Qnsp 135

ons? ij5

ans ias

Qesp ins

Qesp 150

oesf iks

Desp 160

jesp 165

Q⿻5 5P 170

gesp 175

gesp 190

Qesp 185

gesp 190

gesp 195

0esp 230

Quse 205

gesp 210

Qusp 215

gesp 220

ass 225

Qese 20

Qesp 235

-5? 240

Qusp 205

oes? 250

QESP 255

Qesp 260

Q4SP 265

QESP 270

Qesp 275

oesp 280

ousp 285

Qesp 290

oesp 295

Qusp 306

cesp 305

gese 310

gesp 310

gap 315

oase 320

Qusp 125

case 130

oesp 335

oesp 360

Qesp 305

Qusp 350

Qusp 354

aese 360

Qesp 365

Qes? 370

Qesp 375

cosp 380

QuSP 185

QusP 390

ouse 395

Qusp 100

josp 105

zose 10

qusp is

oss 120

asse 25

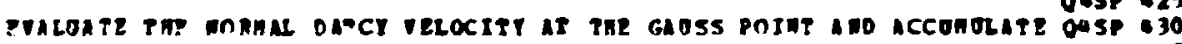

THP INTZGPAL SEAS 


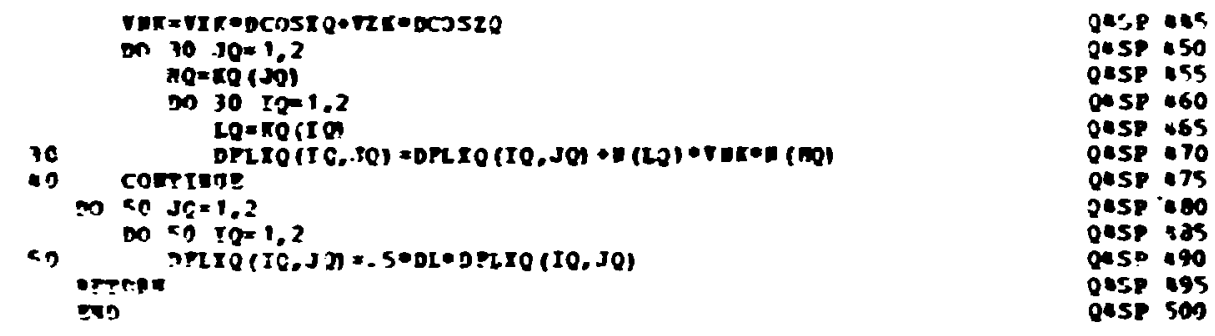

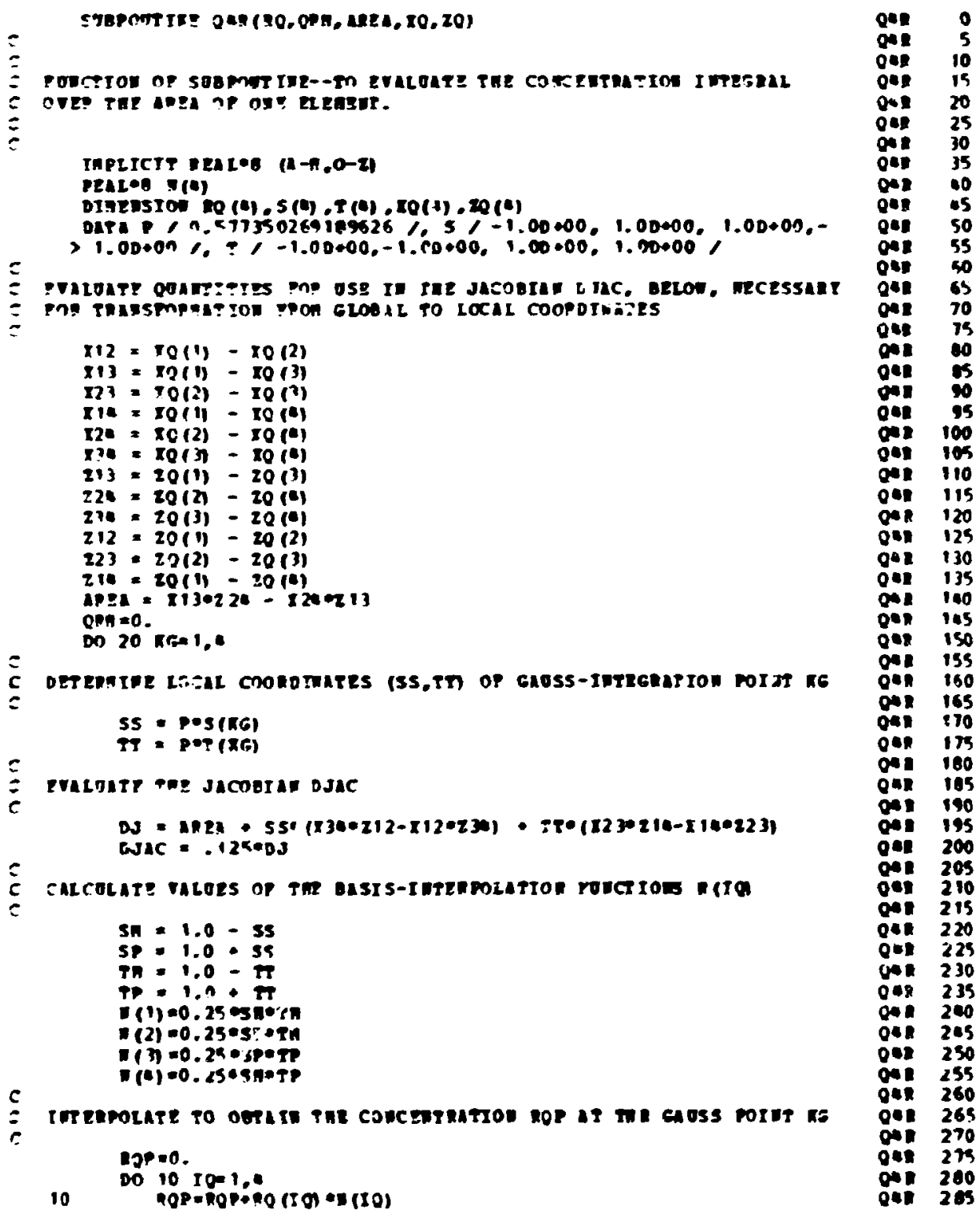




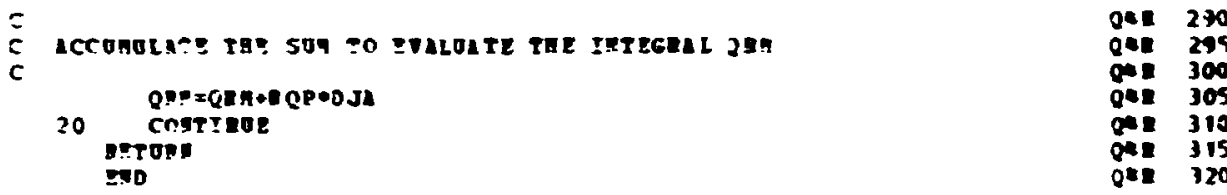

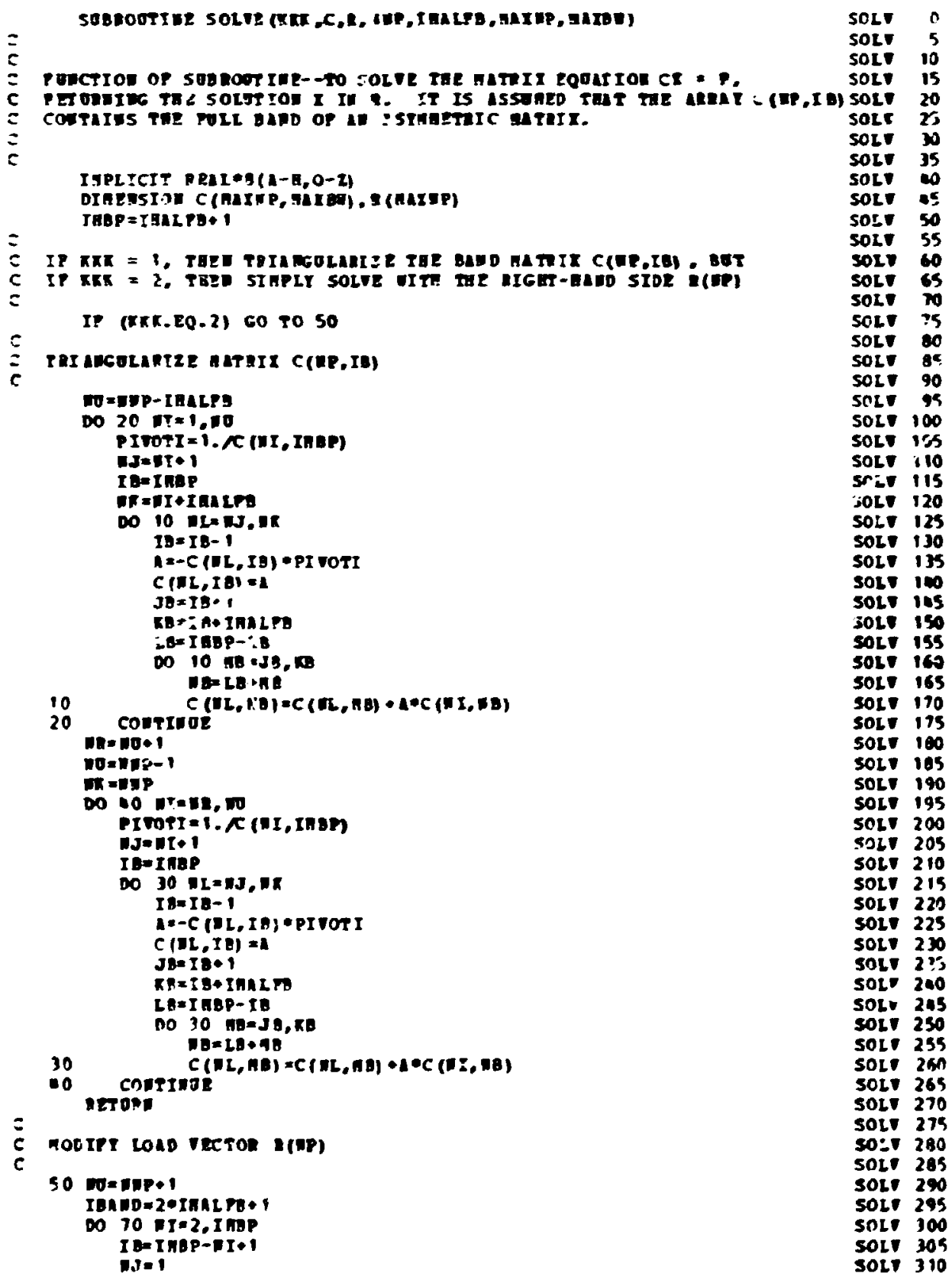




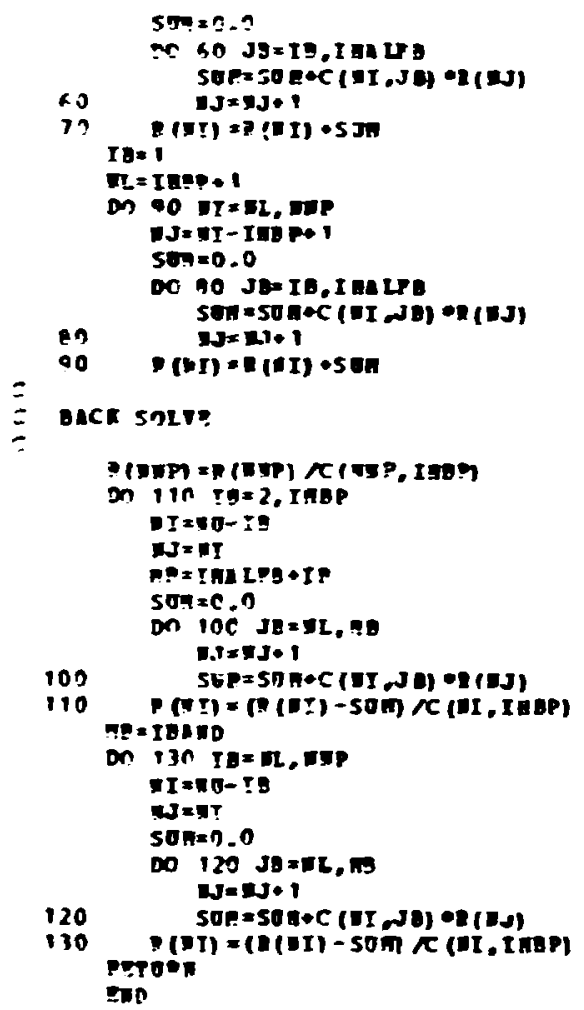

SOL" 3 is

SOLT 320

50L. 325

SOLV 130

soLt 335

SOLT 340

SOLV 3 as

SOL 350

SCLV 355

SOL 369

50t. 365

SIL 376

SOLV 375

SOLT 380

SOLT 385

SnLV 390

SOLV 305

solv 100

Srit 0 os

SOLT 10

SOLT i:s

$50 L=20$

$50 L 1 \times 25$

solv 430

solv 35

SCL : en

SOLT aes

SCLV EST

5021 :55

SOLY 60

SOL 69

SOL 17

SOL .75

SOLT 40

SOLT aAs

SOL 190

SOLT a9s

SOLT SOC 
EENDIX D

DEFINITION OF VARIABLES 
41

ALP

A.M

AMO. AMI

AREA

AT

BBY.YPP)

BBI. BBJ

BFLX(NP)

BFLXP(NP)

$C(N P, J B)$

CHNG

$\operatorname{DCOSX}(\mathrm{MP})$

DCOSXB(MP)

DD

$\operatorname{DCOSZ}(\mathrm{MP})$

DCOSZB (MP)

DFLMAX
Longitudinal dispersivity of the material u ithin an element

Moditied coefficient of compressibility... $1 * 1-1$ ).

Molecular diffusivity...L**? T.

In SLRF. ariables used to establish the signs oi the direction: cosines.

Diagnustic element variable ... $1 * 2$.

Transierse dispersivity of the material within an element.

Array for storing the constant material concentrations for Dirichlet boundary conditions....M $I * *\}$.

Variables used in automatic generation of constant-concentration Dirichlet boundary conditions BB...M I.**?

Normal boundary flux attributable to node $\mathrm{NP} . . \mathrm{M} \mathrm{L} . \mathrm{T}$

Same as BFLX but for previous time step.. M I. T.

Assembled matrix $\boldsymbol{W} * \mathrm{~B}+\mathrm{A}$ DEI.T where $B$ is the spatial operator matrix and $A$ contains the coefficients associated with the time-derivative terms...L**2 $\mathrm{T}$.

Multiplier for increasing the time increment.

Direction cosine of outwardly directed surface normal (with respect to the $x$-axis) for secpage surface as a function of the boundary element.

Same as DCOSX(MP). but for all bounding surfaces.

Effective molecular diffusivity $A M * T A C \ldots L * 2 T$

Direction cosine of outwardly directed surface normal (with respect to the 7-axis) for seepage surface as a function of the boundary element

Same as $D(\operatorname{OSZ}(\mathrm{MP})$, but for all bounding surfaces.

Ma:imum value of DEI,T...T. 
DEI.T

IDEI II

DH

DFI.XI

DFI.XJ

DFLXQIQJQI

I)H(NP)

DHK

INHQ1QI

[D]

[)]I

DI. (MP)

DI $B(M P)$

I)XXIQJQ;

I) $.71(10.19)$

I)P( $(P)$

I)RX
Time increment...T.

I DEI.T..T**(-I).

Difference in nodal numbers to be used in automatic generation of nodal coordinates.

Surface integral over product of normal nux and basis function N(IQ) for an element...M T $\mathrm{L}$.

Surface integral over product of normal tlux anc basis function NiJQ) for an element.... $\mathrm{T}$ L.

Matrix containing suriace integrals for an element side used in seepage boundary conditions... M T I.

Time derivatise of the pressure head...L T.

Time derivative of the piessure head at Gauss-integration point K(j...:

Time derisative of the r,ressure head at node IQ of an element...I. $\Gamma$

Jacohian times $\mathrm{X} \ldots \ldots * 2$.

I I) J..I $: *(-2)$.

I,ength of seepage soundary side of element MiP...L.

I ength of any houndary side $f$ element MP...L.

Derivative of b-sis function $\mathrm{V}(\mathrm{IQ})$ with respect to $X$ at position JQ... $* *(-1)$.

Derivative o! basis function N(IQ) with respect to $Z$ at position IQ $\ldots 1 * *(-!)$.

Array lor storage of constant leumann boundary conditions....M I. $\mathrm{T}$

Derivative of concentration $R$ with respect to X...M L** 1 .. 
IDRZ

i) $[\mathrm{H}(\mathrm{M} . \mathrm{IQ})$

ID.X

DXX

DZZ

IDZ

DZ7.

EI. EJ, EK

EL

FI,OW(I)

F.iK

FWNI, FNSJ

FPATE(1)

FX(M.IQ)

FXK

F..(M.1Q)
Derivative of concentration $R$ with respect to $7 \ldots .14$.** $!$

Time derisatise of moisture content ..L**3 I.*? I .

Incremental distance in X-direction.....

XX-component of the dispersion tensor...1.*2 $\mathrm{T}$.

XZ-component of the dispersion tensor...I**2 1 .

incremental distance in Z-dirction...1.

ZZ component of the dispersion tensor... $1 * 2 \mathrm{~T}$.

Normal fluxes to be useci ior Tux-iype Teumann boundary conditions....M $[* 2 \mathrm{~T}$.

Length of an element side where flux-type boundary condition is applied...L.

Flows across the system boundary during time DELT through nodes having constant Dirichlet conditions $(l=1)$, through nodes having constant licumann conditions $(I=2)$. by seepage $(I=3)$. due to riumerical losses $(I=4)$. and througk all boundary nod ss $(I=5)$. Flow (6) is the net change in adsorbed material occhiring during time DEI.T. FI.OW(7) is the net change in dissolved material occurring during time Dé.T. arid Fl.OW(8) is the net material loss via radioactive decay in the same time interval....M $\mathrm{I}$.

Normal flux at Gauss point $K G$ used in surface integration...M $L * * 2 T$.

Cemponents of the material fux normal to the boundary surface at nodes $\mathrm{NI}$ and $\mathrm{NJ}$. respectively...M $\mathrm{I}, * 2 \mathrm{~T}$.

Flow rates at a given time corresponding to FI.OW(I)...M L $\mathrm{I}$.

$X$-component of material flux ..M $1, * 2 T$.

$X$-comporient of material flux at Gauss-integrition point KCi along the boundary side of an element... $1 * 2 \mathrm{~T}$.

Z-component of material flux...M L**2 T. 
HZK

HoNP,

HP(XP)

HI(NP)

IB. JB. elt

IB.AN)

IE.M.IQ)

IHAIII B

IH HP

IXC

10. 30.

101

ISI.MP.I)

ISB(MP.I)

ISTOP

ISI. 1.52

III!

.101

KI)
Z-component of the material thux at (iauss-integration. point KG; along the boundary siate of an element. It $1 . * 2$ ? 1 .

Pressure head at current tirne sep...1.

Pressure head at next presious tim: step...1.

Total head...i..

Indices ranging over the band width of the cietfickent matrix.

Band width of ascembled coelficicit matrix

Element identification array. The entry in $10=5$ ideritltes th: material t:pe for elemem nt M.

(IBAND-1, 2.

IHAI.FB - I

Increment in nodal number to be used in automatic generation of boundary corditions.

local node or basis function identiter haseng values if 1.2 .5 or 4 .

10 - 1. a local node index.

Surface identification array for seepage eleme.nts. Valuev $I=I$ and 2 denote gioisal node numbers of the surface side. and $I=3$ ar.d 4 dencte the corresponding local node numbers.

Same as IS(MP.I) hut for all boundar, elements.

Inde: used to count data crrors.

Global node numbers defining an ekment side on uhreli xecpage houndary conditions are io be applied.

Index for simulation time.

IA + I. a local nonde indes.

Distributon cieflicien of an element... $* 3$ W. 
KDIG

$\ddot{n}$

KIIC

RKI:

KOLT

KPR(ITM)

KPRO

KSS

KSTR

KVI

I.AMBDA
Dagnostic output tabke counter

Identifer of the tour (iauss incegration points within ach element.

Incrementation sontrol used for automatic zenerstion of buundary conditions.

In SOLVE. index desizating function .. be performed. Parameter KYK for triangulaisation and KKh for hackward substitutipn.

Output table counter.

Printer control for transient problems similar to KPRO as a function of the time under ITM. ised to output desired information for sach time increment.

Printer control for steady state and initial conditions. If KPRO $=0$. 'snly the now variables Fl.OW. FRATE, and TFL.OW are output. IF KPRO $=1$, then concentration variables are printed. If $K P R O=2$. material fuxes and those variables mentioned previously are printed. If KPRO $=3$. then water ontents and Darcy velocities are also output. Finally. if KPRO $>$ 3. all of th: variables mentioned proviously plus pressure heads and total heads are output.

Steady state control. If $\mathrm{KSS}=0$. the steady-state solution is obtained. If K.SS $=$ I. the transient solution is obtained.

Control parameter for storage of output on auxiliary storage (tape or disk). If KSTR $=0$. there is no isvage, but if it does not equal 0 . there is storage on logical init 2.

Darcy velocity input control. If $K V I=0$. there is no input and the velocities are set equal in 0 . If $K V I=1$, time independent velocities are input If $K V I=2$, time dependent velocities are ..pput for each time step. For $K V I<0$ card input is used. and for KVI $>0$ auxiliary storage (taps or disk. logical unit 1 ) is used. In the laiter cise nodal positions and element definitions are also read from the magnetic tape.

Radioactive dccay constant...T**(-1). 
I.I

M. MI. MJ. MK

MAXBW

MA:DIF

MAXFI.

MANMAT

MAXXP

MAXNTI

MINC

MNI

MODI.

MP. MPI. MPJ

MIVP

N(IQ)

NBC

NBE(MP)

NBEI.

ICM

No

NEI.

MIAY
Yodal number increment to be used in automatic generation of elements.

Ekement number

Maximum vaive of IBA.XD.

Maximum nodal difference for all ekments.

Maximum number of elements.

Maximum number of materials.

Maxim:um number of nodal points.

Maximum number of time increments.

Increment in element number.

Moxinum nodal diffe ence for a given element.

Number of ekements per laye::

Compressed ekment index.

Material type.

Basis vector for node 10 .

Number of constant Dirichlet boundary conditions.

Array of bound2.y ekment numbers.

Number of boundary ekments.

Number of ekments with corrected material properties.

Viodal differer:x.

Number of ekments.

Number of layers of elements in regular part of grid. 
MMAT

NMP:'M

II

N.P

NP. N. N. etc.

NPIXC

XPMIX. IPMAX

NPN(XPP)

IPP. NPPI. NPPJ

NPPMAX

NPPROB

NPROB

NPROBM

NPST(NPP)

NPTST(NPP)

NST

NST:

NTI
Number of different materink.

Nuraber of material properties per material.

Y(1QF) NJQ

Number of nodal points.

Yodalpoint number.

Yodalpoint increment used in automatic generation of Vewnenan houndary conditions.

Integer variables used in autumatix peneration of liwinara boundary conditions.

Array for soring the node mushers where constant Dirichlet boundary conditions occur.

Compressed nodal-point index.

Nuniber of nertal indices in unpacked array. NPST.

rutput contrcl integer.

Problem number.

lummy tariable read from magnetic upe enerated by moisture-tmansport code.

Nodal points at which constant-flux $\mathrm{N}$ tann boundary conditions are applied

Absoluf: nods index as a function of compressed index for serpege brundary nodes.

Number of ekment sides on which nux-type Neumaan boundary conditions are, applied.

Number of nodes at which thuxtype bejndary conditions are applied.

Number of time increments. 
IIM

NIstiMPI

MISI

MII

II

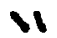

?

PIISIS

PMA!

Inst

PRCHY I I P.I,

QAis)

Givisus

(I)

45x

(NI)

U

Q1.

Ot'
Ihumen sarvak read trom magactix upe genctaked by maniuretirapon cuds

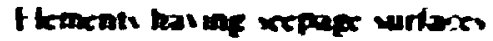

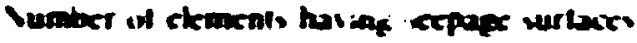

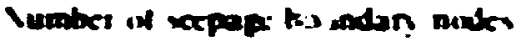

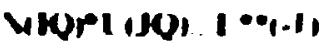

WNorlijor. 1 -

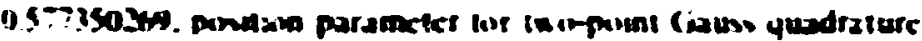

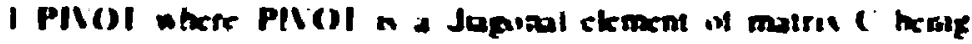
uxed in the matris reduction 1 "थ?

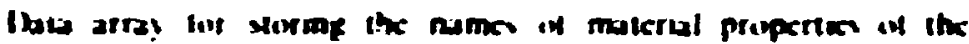
wi! wsem

Pisinat! ul an element

Vateral propert! I her wil ispe Mif if

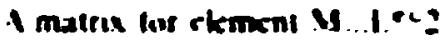

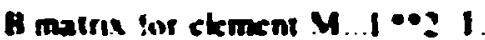

I ual adsurbed natcetal al current ime step... M I

- Wusrhed materal wnhin ckement M. V I

I otal adwithed malesal al nest pres wuss lime vlep.... 1.

Iotal matsral last $m$ radiaacine deca! at curnent ims sep.... 1 .

Ioyal of materal in sold phase and materal in lingud phase w thin element $\mathrm{M} . . \mathrm{M}$ I.

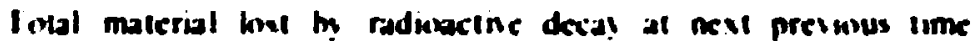
sep...M 


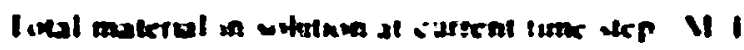

QRM

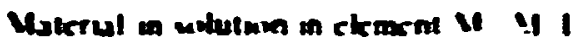

URP

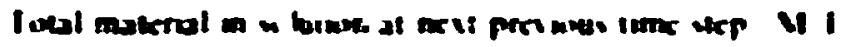

$R_{1} \mathbf{N P}_{1}$

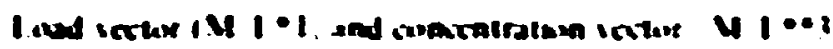

R()

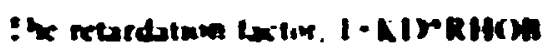

Rikn:

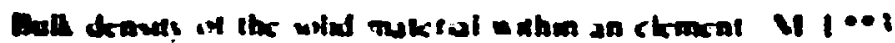

RPVP

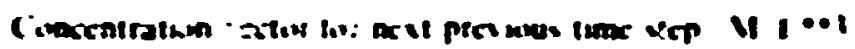

RQNivi)

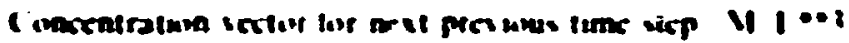

RUP

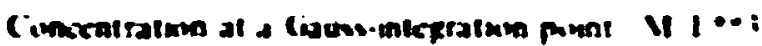

vivi. is

I aral Vimordingles

SW

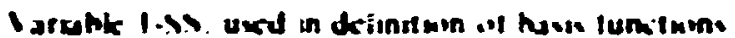

$\mathbf{s}$

$\sin (1)$

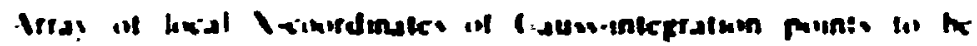
und in thowidan inkpratum

SI 1

lirable und in malfil ceduciun

IINo. II

I ocal / romondinales

$|1|$

Iorrements

ItIowill,

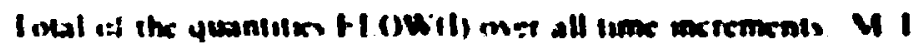

(H.M.NO)

Womtuec conten! at the nidks of each elemeat for corrent ione sep $1 \bullet 11 \bullet ?$

IIIK

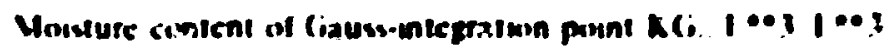

IHPM.KI

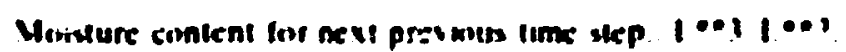

IHONIOI

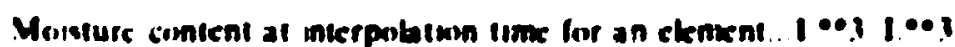


$1114,4.14)$

IIII

III $\mathrm{U}$

HIIII)

IIII Unl1

IV

$14+1$

If

II Mnt.1

I II)!

11

11

11

IIUN

In

$|\mathbf{k}|$

11

111.111

11

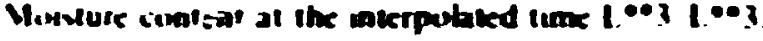

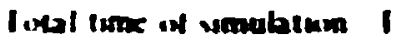

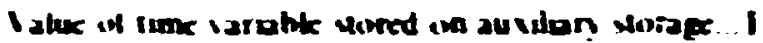

Aras 'if t:tk of the Mablem

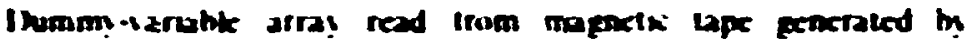
monduretranspert ide

I araink 1-11. und in defianture ot hasn funcemus

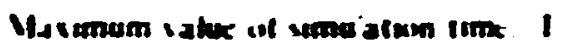

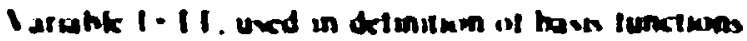

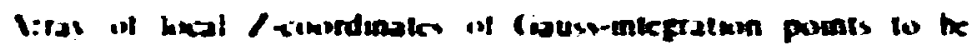
uod in musuding anteftainon

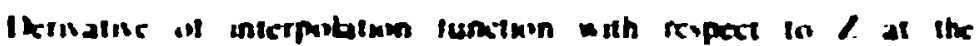

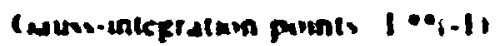

$1,10, * 150,1 \cdot 1-1$,

$110,11011 \cdot 0,-2$

$110, \cdot 110,1 \cdots(-2)$

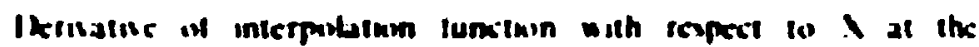
(cauve-iniczratuon meents. $1 \cdot \bullet-1$ )

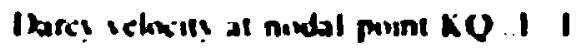

| |K. I |

vilup 1.10$) \ldots+1 \cdot 11$

Cimpuncents of the lasen Irkx'!! normal to the surlace nodes $V I$ and $N 1$. respertinch... I

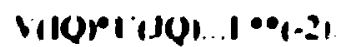


IV

IXIM.IQ1

VXx. 12K

VXPIM.101

NTO1Q)

IXViM.10)

IZIM.IO)

|ZPIM.1Q)

Izoilor

VZWiM.K()

u. wi, w2

X(XP). Z(XP)

Xo. ZO

XI.. XI3. elc.

xQ101

Z12. Z13. etc.

Z.(10) vilorvisopi...1.en -2)

Xromponent of the Dancy velucity at the modes of each ekement for the current time step... L. I

Danc: velucity components at (auss untegratum point ho I I

Xeomponent of the Darcy sekxity for the next previsus ume step.... T.

Xcumponent of the Darcy velocity at the interpolated tume step for an eiement...L. T.

Xecomponent of the Darcy velocing at the interpolated time...L. I

Z-component of the Dares velocilt at the nodes of each element for the current time step...I. T.

Zcomponent of the Iares velocity for the nexl previous time step.... I. I.

7 compunent of the Darcy selocity of the interpolated time for an ekmeri..... T.

Z-component of the Darcy vehxit! at the interpolated time...I. I.

Time-integration parameters.

X- and Z-coordinates of mode ..P...I.

Fake coordinaten used to determine the signs of $\therefore$-- direction cosines...1.

Quantities $X(J)=X(1) \cdot X(J) \ldots I$.

X-coordinates of the nodes of a quadribiteral ekmemi... I..

Quantities $\mathbf{Z I J}=\mathbf{Z}(\mathbf{I}) \cdot \mathbf{Z}(J) \ldots \mathbf{R}$

Z-coordinates of the nortes of a quadritateral ekement...I. 
$19:$

APPENDIX E

Data input glide 


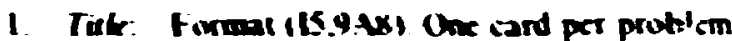

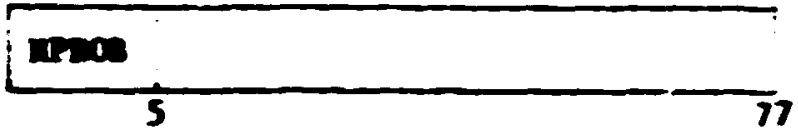

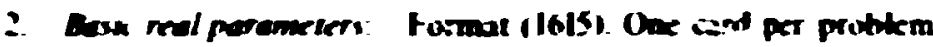

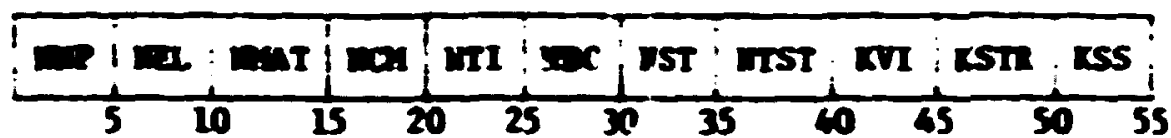

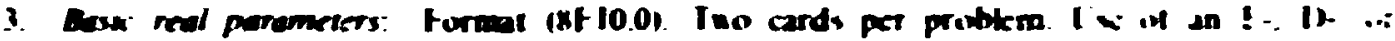

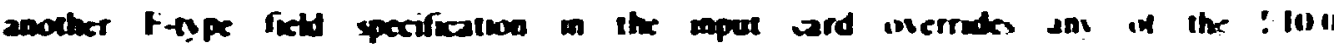
specifications of the tormal.

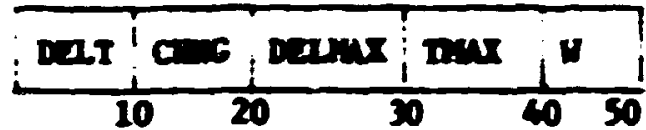

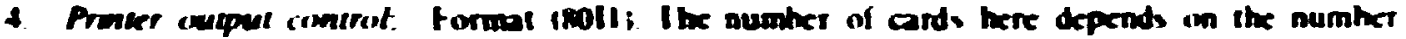
of time acremeats VII.

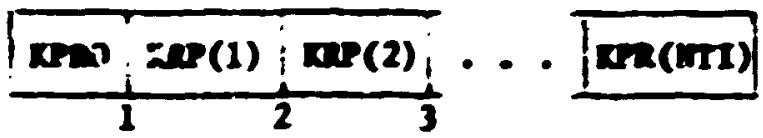

5. Maierial properives: format ixf 10.0). A total of XMAI cards. or: for each mater:..

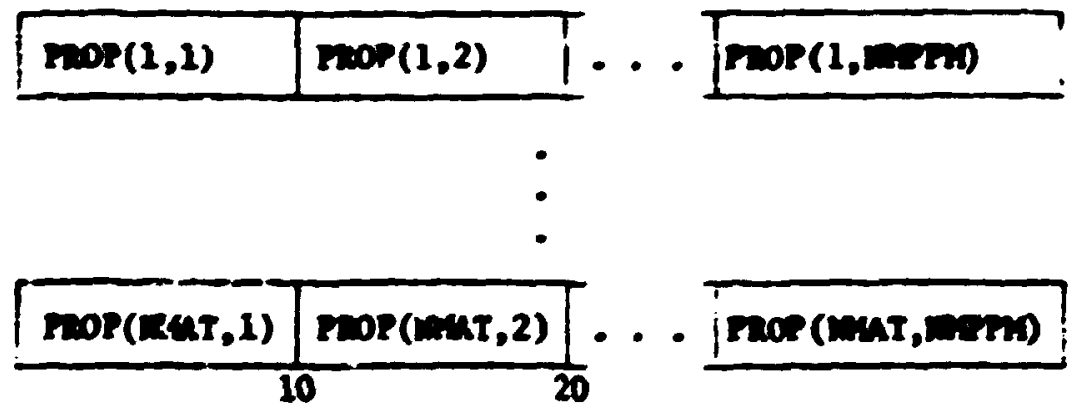




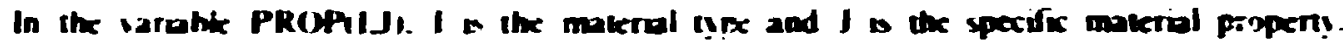

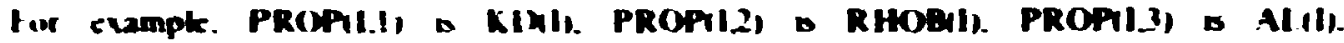

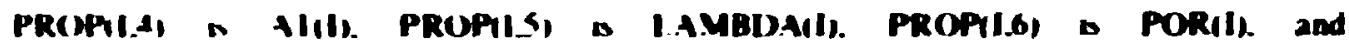
PRCPPI. MPPM, s Al PII. I.MPPM=7 s curreath prescribed on a dala stakencat in revutione $\mathrm{M}+\mathrm{I}$,

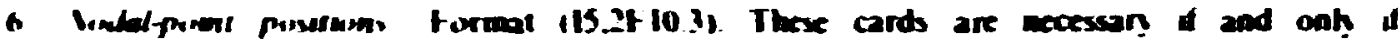

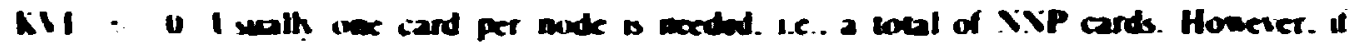

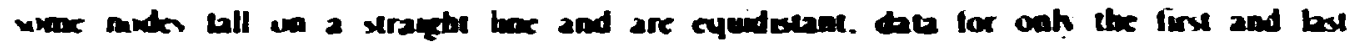

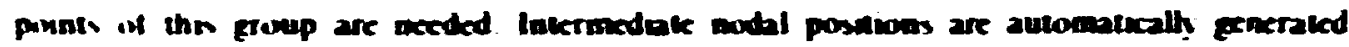
In Irear unctpitatuan

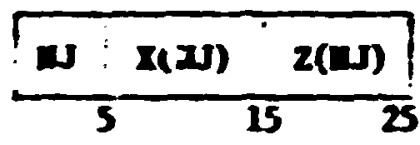

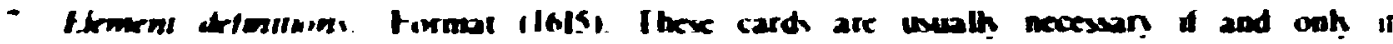

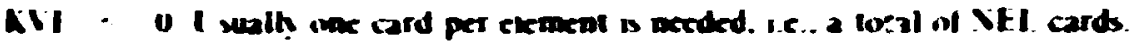

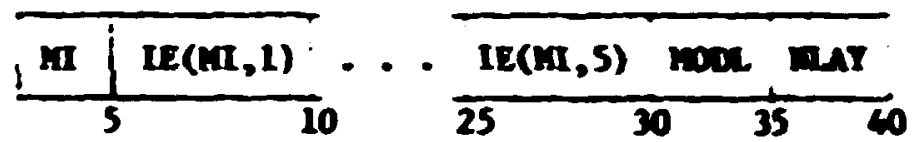

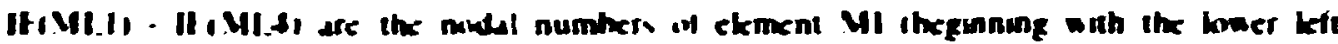

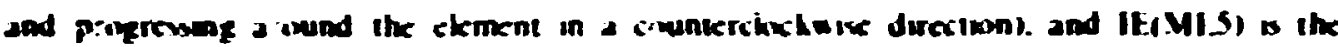
matera I!pe VIYP for rectangular hich, of elements hav ung the same makeral typ

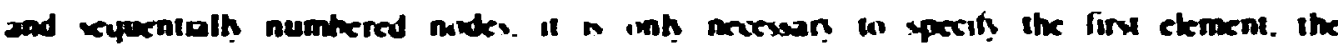

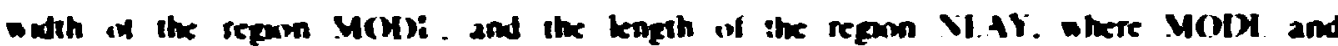
II are meawned in skments fkment numbering proceeds must raputh abong the

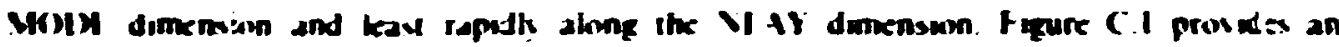

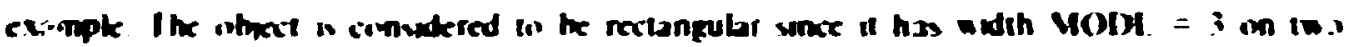

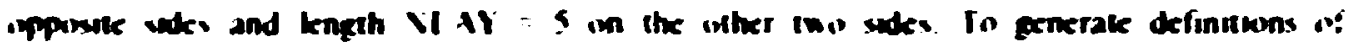
ekment. : through is automaticalh. including muh corner mode dentification and materual itpe. only ine cord is necenwn

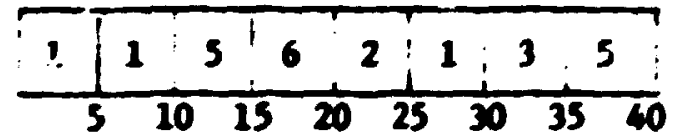




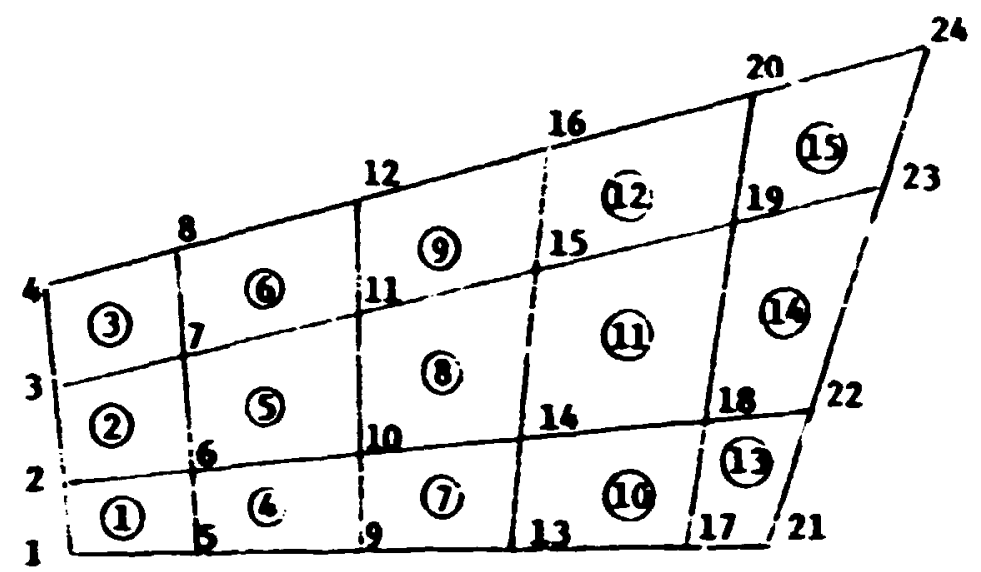

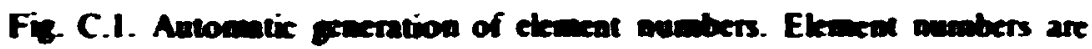
cincled to distingind thes from nodal nesmbers.

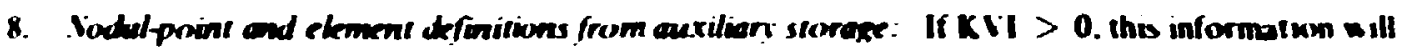
be obtained irom auxilian suorage (magnetic upe. for example). Two unformalted RE.AI) statemrats are used for this purpose:

\section{READIXTITLE.M(I).l=1.9).XPROBM.N.XP..NEI...TIM

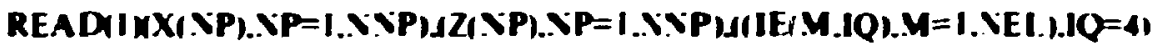

Tose on auxilian storoge wnits: logial unit I is used for input if KVI $=0$. and logkal unit 2 is used for outpul if KSTR $=0$. Proper identification of these units must be made in the job control banguage if either of these two options is used.

9. Moterial correction: Format (1615). Cards are required bere only if XCM $>0$. In many cases one card is required per material change. However. in those cases where numbers of the affected elements range from a lower limit of MI to an upper limit of MK with an increment MIXC. automatic correction ma: be used. Fields $\mathbf{M K}$ and MI:iC are keft blank if the automatic generation facility is not used.

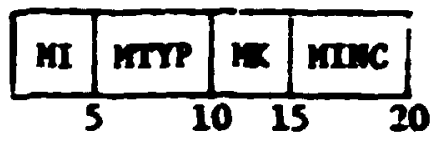




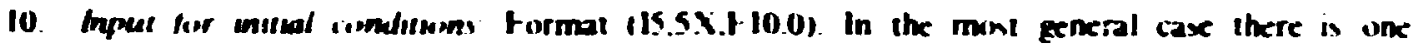
card per newte. I.e. a lokal of XVP cards

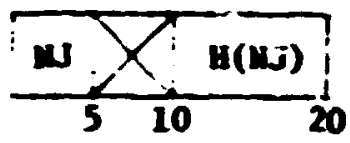

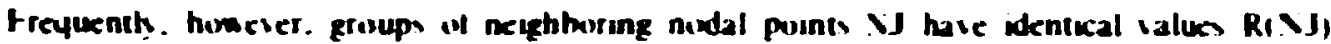
if a gap is recognued in the unput seyuence of nodal numbers. the initial concentrations are assunied Is he dentical to the concentration at ine hoer houndan of the gap. For cumple. it ino neighturing cards of the torm.
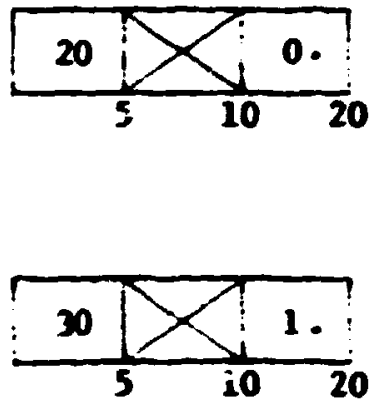

nete enrountered. nodes $? 1-2 y$ would be assigned values $R=0$

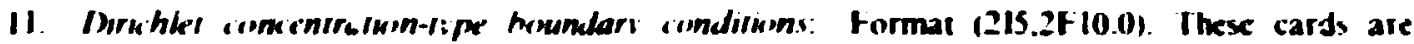

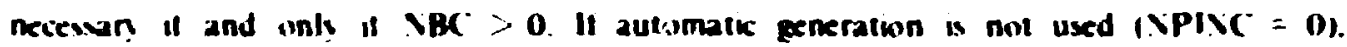
VBC cards are reyuired o; the form:

\begin{tabular}{|c|c|c|}
\hline $\operatorname{MH}(\operatorname{MPP})$ & NPIXC & $\mathbf{D B}(\mathrm{NPP})$ \\
\hline
\end{tabular}

Ii XPIXC ; 0. autumatic generation proceeds in the same manner as described for Data Set II. I hat is, an algebrac sequence is huilt on the noda! number 1.1 of the card immeduately preceding. and each such node is given boundary condition BB of that card. 
12. Veumenn flux-type boundery conditions: Format (315.5X...F 10.0). Cards of this type must be ured if and only if NST $>0$. Vsually a number of cards equal to NST must be ised. However. if some of the KINC are greater than zero. seme boundary conditions wili be generated internally. and $\mathrm{NST}$ cards will not be necessany.

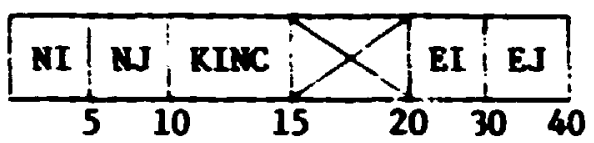

If KIXC $>0$. then the nodal-point increment is formed from $x$ and $X J$ of the insmediately preceding card:

$\mathbf{N F} \cdot \mathbf{I N C}=\mathbf{N J}-\mathbf{N I}$

Two segue aces are formed:

$$
\begin{aligned}
& \mathrm{NI}+\mathrm{NPINC} . \mathrm{NI}+2 * \text { NPINC } \\
& \mathrm{NI}+\text {.NPINC. } \mathrm{N}+2 * \text { NPINC }
\end{aligned}
$$

Both are terminated when the largest integer is reached that is kess than both current values of $\mathbf{N I}$ and NJ. Corresponding nodal points for these two sequences define a surface. Quantity EI is the dot product of the flux at NI with an ouiwardly directed unit vector normal to the element side (NI.NJ). A similar definition holas for EJ.

13. Seepage surface elements: Format (1615). input is necessary here if and only if XTST > 0. Typically, one card is required for each side of each element on which such a bourdary condition is to be applied.

\begin{tabular}{|l|l|l|l|}
\hline MTSE (MP) & IS (MP, 1) & IS (MP, 2) & KINC \\
\hline 5
\end{tabular}

However. if KINC $>0$, automatic generation is employed in the following manner. Nindal-point-number and element-number increments are formed from information on the input card immediately preceding the current one:

NPINC $=15(M P, 2)-1 S(M P, 1)$

MINC $=$ NPIN" -1 
where the vertical bars denote absolute value. A seyuence of ckment numbers is then obtained:

$$
\begin{aligned}
& M=\operatorname{IRSE}(M P) \text { (previous card) } \\
& \operatorname{VRSE}(M P+1)=M+M I N \\
& \operatorname{VRSE}(M P+2)=M+2 * M I N C
\end{aligned}
$$

The sequence is continued until the largest element number is encountered that has a value kess than NISE of the current card. Corresponding nodal point sequences are also gencrated:

$$
\begin{aligned}
& \mathbf{N I}=\mathbf{I S}(\mathrm{MP} . \mathbf{I}) \text { (previous card) } \\
& \text { IS(MP+l.l) }=\mathbf{M l}+\text {.XPINC } \\
& I S(M P+2.1)=N I+2 * N P I N C \\
& \mathbf{X J}=\mathbf{I S}(\mathbf{M P} . \mathbf{2}) \\
& I S(M P+1.2)=N J+\text { NPINC } \\
& \text { IS(MP+2.2) }=\mathrm{NJ}+2 * \mathrm{NPINC}
\end{aligned}
$$

14. Card impul of pressure heads al time $t=0$ : Format $(8 F \mid 0.0)$. If KVI $<0$. NYP values of H must appear as follows:

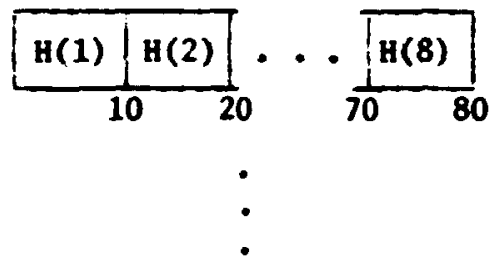

H(NNP) 
15. Card input of water contents at this rime $t=0$ : Format (8F In.0). If $\mathrm{KVI}<0$. NEl. cards are required:

\begin{tabular}{|c|c|c|c|}
\hline $\operatorname{TH}(1,1)$ & $\mathrm{TH}(1,2)$ & $\mathrm{m}(1,3)$ & $\operatorname{TH}(1,6)$ \\
\hline
\end{tabular}

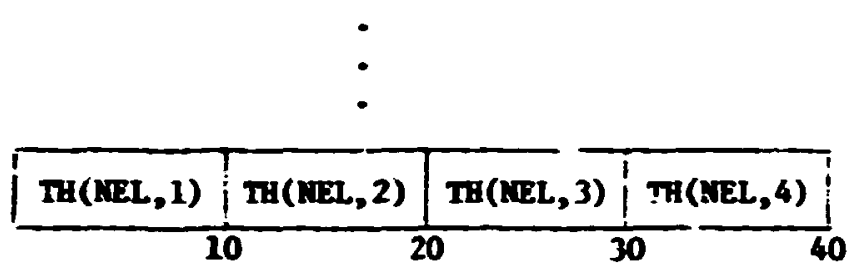

16. Card inpul of Darcy velocities a: time $t=0$ : Format (8Fi0.0). If $\mathrm{hVI}<0$. NEl. cards are required:

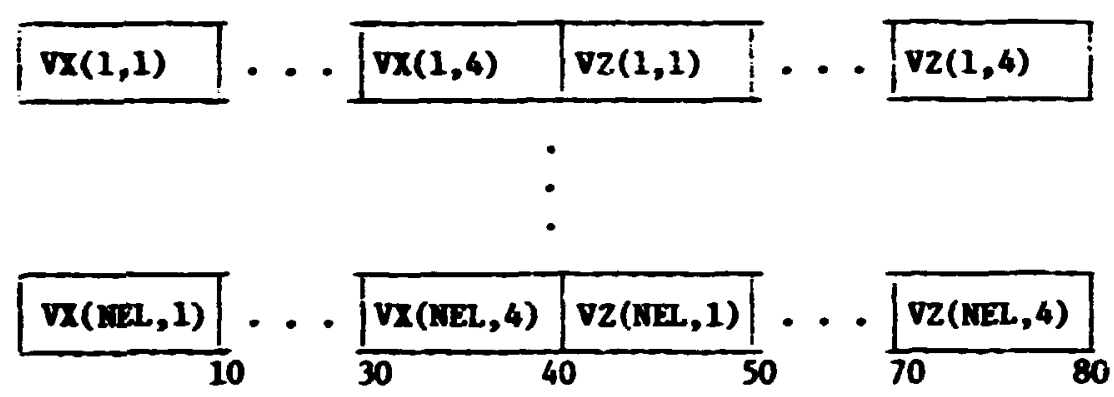

17. Input of moisture-transport variables from auxiliary storage at time $t=0$ : If $\mathrm{KVI}>0$. 2n unformatted READ statement is used.

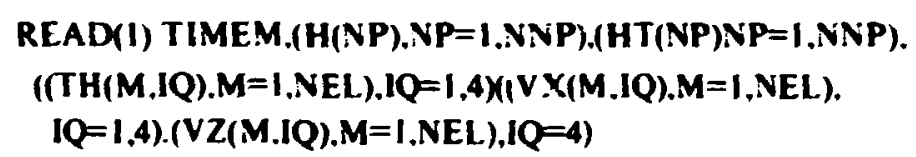

18. Card is.put of moisture transport variahles for times $1>0$ : When $K V I=-1$, a steady-state moisture transport is assumed and no upda:e is required for $t>0$. If, however, $\mathrm{KVI}=-2$, transient meisture transport is assumed, and three sets of cards like those shown in paragraphs 14,15 , and 16 are required for each time step.

19. Input of moisture-transport variables from auxilian storage for times $1>0$ : When KVI $=1$. a steady-state moisture transport is assumed and no update is required for $1>0$. If, however, $K V I=2$, transient moisture transport is assumed, and moisture-transpor: variables are read from auxiliary stomge by the unformatted READ statement of Data Set 17. 